\title{
DYNAMIC RESPONSE VALIDATION OF CUBESATS THROUGH TESTING AND FINITE ELEMENT ANALYSIS
}

\author{
A Thesis \\ presented to \\ the Faculty of California Polytechnic State University, \\ San Luis Obispo
}

\author{
In Partial Fulfillment \\ of the Requirements for the Degree \\ Master of Science in Mechanical Engineering
}

by

Peter Rivera

June 2019 
(C) 2019

Peter Rivera

ALL RIGHTS RESERVED 


\section{COMMITTEE MEMBERSHIP}

TITLE: Dynamic Response Validation of CubeSats through Testing and Finite Element Analysis

AUTHOR: Peter Rivera

DATE SUBMITTED: June 2019

COMMITTEE CHAIR: Xi Wu, Ph.D.

Professor of Mechanical Engineering

COMMitTeE MEMBER: Eltahry Elghandour, Ph.D.

Associate Professor of Mechanical Engineering

COMMitTeE MEMBER: Mohammad Noori, Ph.D.

Professor of Mechanical Engineering 


\begin{abstract}
Dynamic Response Validation of CubeSats through Testing and Finite Element Analysis

Peter Rivera
\end{abstract}

During a rocket launch, satellites withstand large dynamic forces due to vibrations that are transmitted from the launch vehicle. As such, they must undergo testing to ensure that their function is not impaired by such forces. CubeSats are cost-effective nanosatellites, and like their larger counterparts, undergo dynamic testing for launch qualification. While dynamic analysis can done on a CubeSat alone, other assembly constituents also affect the response, and including them in the analysis can provide insight on the true dynamic behavior of CubeSats. To show this, the work presented here recreates a vibration test setup using Finite Element Analysis (FEA) in order to predict the dynamic response of a CubeSat test assembly. A Poly Picosatellite Orbital Deployer (P-Pod) is used as the housing and ejection mechanism for CubeSats. For modeling purposes however, a simplified P-Pod (test pod) was used, along with a mass model to represent a CubeSat. Sine sweep and random vibration tests were performed for the test pod and mass model. For the FEA simulation, CAD geometry and material properties were imported into Abaqus to solve for the different modes and natural frequencies of the structures. From the FEA models, the first six natural frequencies of the test pod were generated, with an average $7 \%$ error when compared to the experimental data. The analysis was repeated for the test pod and mass model assembly, and the first eight natural frequencies were generated, with an average $4 \%$ error when compared to the experimental data. With the FEA models validated, the FEA template was applied to CubeSat CPX, a concept design structure used to demonstrate the application of the FEA model developed in this work. 
Dedicado a mis padres... por ser el ejemplo vivo de la perseverancia... y en agradecimiento por los sacrificios que tuvieron que hacer para poder darme la vida...

... and to my little sister... who even in my deepest moments of despair... never once let me falter... 


\section{ACKNOWLEDGMENTS}

Thanks to:

- My family and friends, for all their loving support throughout the course of this endeavor.

- My colleagues, for their company and support during those long days in the graduate lab.

- Cal Poly SLO CubeSat/PolySat, for the technical support that made all of this possible, I'm proud to have been part of such an amazing program.

- Chang Kim (Unholtz-Dickie Corp.), for his generous support with the vibration table CAD models.

- My committee, for taking me under their wing and providing invaluable guidance, special thanks to Professor EE for his immeasurable patience and for being a compass in my journey through grad school. 


\section{TABLE OF CONTENTS}

Page

LIST OF TABLES . . . . . . . . . . . . . . . . . . . xii

LIST OF FIGURES . . . . . . . . . . . . . . . . . xiii CHAPTER

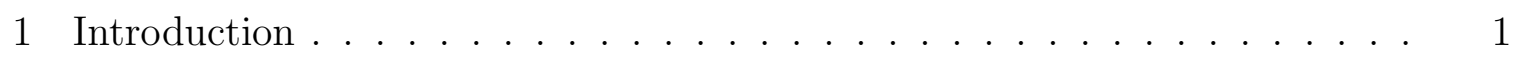

1.1 Background and Scope $\ldots \ldots \ldots \ldots \ldots$

1.2 CubeSat Program . . . . . . . . . . . . . . . . . . 4

1.3 ELaNa . . . . . . . . . . . . . . . . . . . 5

1.4 CubeSat Design Standard . . . . . . . . . . . . . 6

1.5 Poly Picosatellite Orbital Deployer _. . . . . . . . . . . . 8

1.6 Ground Stations . . . . . . . . . . . . . . . . . . . . 10

1.7 Launch Environment and Testing . . . . . . . . . . . . . 11

1.8 NASA GEVS $\ldots \ldots \ldots \ldots \ldots \ldots$

2 Theoretical Analysis . . . . . . . . . . . . . . . . 17

$2.1 \quad$ Nature of Vibration . . . . . . . . . . . . . . . . 17

2.1 .1 Resonance . . . . . . . . . . . . . . . . . . . . . . 19

2.1.2 Free Response of Damped Systems _. . . . . . . . . . . 23

2.1 .3 Linear vs Nonlinear Analysis . . . . . . . . . . . . . . 26

2.1.4 Multiple-Degree-of-Freedom Systems _ . . . . . . . . 27

2.2 Finite Element Approach . . . . . . . . . . . . . . . . . . . . . . . 29

2.2 .1 Structural Dynamics . . . . . . . . . . . . . . 30

3 Design Methodology . . . . . . . . . . . . . . . . . . . . 34

3.1 Concept . . . . . . . . . . . . . . . . 34 
3.2 Modeling the Test Setup . . . . . . . . . . . . . . . . . 35

3.2 .1 Test Pod . . . . . . . . . . . . . . . . . 36

$3.2 .2 \quad$ Mass Model . . . . . . . . . . . . . . . . . . . . . . . . . . . 41

$3.2 .3 \quad$ Fixture Plate . . . . . . . . . . . . . . . . . . . . 43

3.2 .4 Assembly . . . . . . . . . . . . . . . . . . . 46

3.3 Idea Behind a Concept Structure . . . . . . . . . . . . . . . . 49

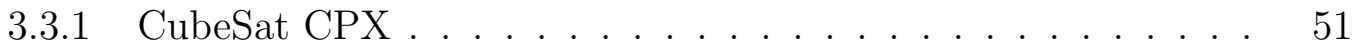

3.3 .2 Assembly . . . . . . . . . . . . . . . . . . . 57

4 Experimental Testing, Results, and Discussion . . . . . . . . . . . . 59

4.1 Vibration Table . . . . . . . . . . . . . . . . . . . . 59

4.2 Measurement Hardware . . . . . . . . . . . . . . . . . 63

4.3 Assembly . . . . . . . . . . . . . . . . . 66

4.3.1 Test Pod Configuration . . . . . . . . . . . . . . . 66

4.3.2 Test Pod with Mass Model Configuration . . . . . . . . . . . 68

4.4 Testing . . . . . . . . . . . . . . . . . . . . . 71

4.4.1 Dry Run (fixture plate) . . . . . . . . . . . . . . . . 71

4.4.1.1 Sine Sweep . . . . . . . . . . . . . . . . . . . . 73

4.4.1.2 Random Vibration . . . . . . . . . . . . . . . 74

$4.4 .2 \quad$ Test Pod . . . . . . . . . . . . . . . . . . . . . 75

4.4.2.1 Pre Sine Sweep . . . . . . . . . . . . . . . . 76

4.4.2.2 Random Vibration . . . . . . . . . . . . . . 77

4.4.2.3 Post Sine Sweep . . . . . . . . . . . . . . . . . 78

4.4 .3 Test Pod with Mass Model . . . . . . . . . . . . . . . . . . 79

4.4.3.1 Pre Sine Sweep . . . . . . . . . . . . . . . . . 80

4.4.3.2 Random Vibration . . . . . . . . . . . . . . . 81 
4.4.3.3 Post Sine Sweep . . . . . . . . . . . . . . 82

4.5 Post-Process of Experimental Data . . . . . . . . . . . . . 83

5 Finite Element Analysis and Discussion . . . . . . . . . . . . . 85

5.1 Early Work . . . . . . . . . . . . . . . . 85

5.2 Finite Element Analysis . . . . . . . . . . . . . . . . . 92

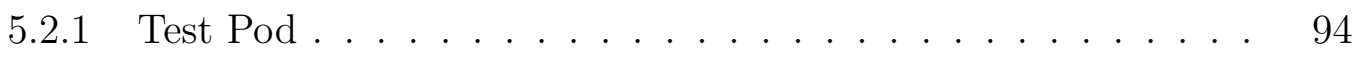

5.2.2 Mass Model ................... 96

5.2.3 Fixture Plate . . . . . . . . . . . . . . . . 97

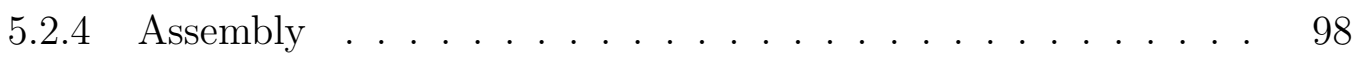

5.2.5 Interactions and Constraints . . . . . . . . . . . . 99

5.2.6 Meshing ....................... 101

5.2.6.1 Element Shape ............... . . 101

5.2.6.2 Element Type . . . . . . . . . . . . . . 103

5.2.6.3 Element Size . . . . . . . . . . . . . . . . 104

5.2.7 Additional FEA Model Parameters . . . . . . . . . . . . 106

5.2.7.1 Properties ................. 106

5.2 .7 .2 Loads . . . . . . . . . . . . . . . 106

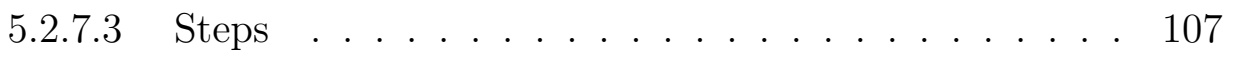

5.2.7.4 Boundary Conditions . . . . . . . . . . . . 108

5.2.8 Supplementary FEA . . . . . . . . . . . . . . 109

5.3 Preliminary Modeling . . . . . . . . . . . . . . . . . 110

5.3.1 Cantilever Slender Beam . . . . . . . . . . . . . . . . 110

5.3.2 Cantilever Solid Square Beam . . . . . . . . . . . . . 111

5.3 .3 Cantilever Hollow Square Beam . . . . . . . . . . . . . . . 112

5.4 Complete FEA assembly of the Test Pod and Mass Model . . . . . . 113 
5.5 Complete FEA assembly of the Test Pod and CubeSat CPX . . . . . 114

6 FEA Results and their Comparison to Experimental Data . . . . . . . . 117

6.1 Results of the FEA models . . . . . . . . . . . . . . . . . . 117

6.1.1 Natural Frequencies . . . . . . . . . . . . . . . . 118

6.1.1.1 Test Pod . . . . . . . . . . . . . . 118

6.1.1.2 Test Pod with Mass Model . . . . . . . . . . . 120

6.1.1.3 Test Pod with CubeSat CPX . . . . . . . . . 121

6.1 .2 Mode Shapes . . . . . . . . . . . . . . . . . 123

6.1.2.1 Test Pod . . . . . . . . . . . . . . 123

6.1.2.2 Test Pod with Mass Model . . . . . . . . . . . 125

6.1.2.3 Test Pod with CubeSat CPX . . . . . . . . . . . 127

6.1 .3 Damped Response . . . . . . . . . . . . . . . . . . . . 132

6.1.3.1 Test Pod . . . . . . . . . . . . . . . . . 132

6.1.3.2 Test Pod with Mass Model . . . . . . . . . . . . 134

6.1.3.3 Test Pod with CubeSat CPX . . . . . . . . . . 137

6.2 Comparison of Experimental and FEA results . . . . . . . . . . 139

6.2.1 Test Pod . . . . . . . . . . . . . . . . . . . 139

6.2.2 Test Pod with Mass Model . . . . . . . . . . . . . . . . . 143

6.2.3 Test Pod with CubeSat CPX . . . . . . . . . . . . . . 147

7 Conclusion . . . . . . . . . . . . . . . . . . . . . . 151

7.1 Summary . . . . . . . . . . . . . . . . . 151

7.2 Learnings . . . . . . . . . . . . . . . . . . . 153

7.3 Notes and Recommendations . . . . . . . . . . . . . . . . . 154

7.4 Next Steps . . . . . . . . . . . . . . . . . . . . 155

BIBLIOGRAPHY . . . . . . . . . . . . . . . . . 156 


\section{APPENDICES}

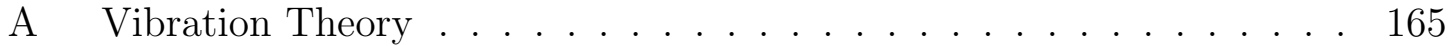

B Finite Element Theory . . . . . . . . . . . . . . . . . . 186

C PCB Accelerometer User Manual . . . . . . . . . . . . . . . . 211

D Mesh Convergence Studies . . . . . . . . . . . . . . . . 228 


\section{LIST OF TABLES}

Table

Page

4.1 NASA GEVS random vibration test levels. ${ }^{40} \ldots \ldots$. . . . . . 72

5.1 List of material properties used for FEA models. . . . . . . . . . . 106

5.2 Mode 1 and 2 of a cantilever slender beam. . . . . . . . . . . . . 110

5.3 Mode 1 of a cantilever solid square beam. . . . . . . . . . . . 111

5.4 Mode 1 of a cantilever hollow square beam. . . . . . . . . . . . . . 112

6.1 Comparison of natural frequencies in the two studies performed for the empty test pod FEA assembly. . . . . . . . . . . . . . . . . 119

6.2 Comparison of natural frequencies between experimental and FEA results for the empty test pod. . . . . . . . . . . . . . . . . . 140

6.3 Two prominent ASD peaks in the random vibration of the empty test pod. . . . . . . . . . . . . . . . . . . 142

6.4 Comparison of natural frequencies between experimental and FEA results for the loaded test pod. . . . . . . . . . . . . . . . . 144

6.5 Three prominent ASD peaks in the random vibration of the loaded test pod. . . . . . . . . . . . . . . . 146

6.6 Natural frequencies of the PolySat and CPX structures. . . . . . . . 148

6.7 Prominent ASD peaks of the PolySat and CPX structures. . . . . . 150 


\section{LIST OF FIGURES}

Figure

1.1 Satellites experience strong vibration forces during the launch phase

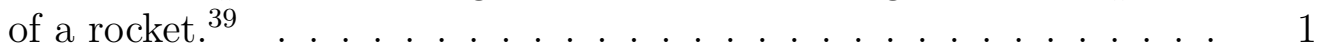

$1.23 \mathrm{U}$ CubeSat in orbit. ${ }^{74} \ldots \ldots \ldots \ldots \ldots$

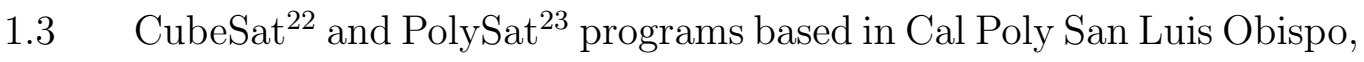
California. . . . . . . . . . . . . . . . . 4

1.4 Snapshot of 1 U CubeSat drawing. ${ }^{22} \ldots \ldots \ldots . \ldots$

1.5 Size comparison of various CubeSat design options. ${ }^{69} \ldots \ldots$

1.6 GRIFEX CubeSat developed by the University of Michigan with NASA ESTO and JPL. ${ }^{38} \ldots \ldots$. . . . . . . . . . . . . . 7

1.7 Poly Picosatellite Orbital Deployer (P-Pod) developed by the Cal Poly SLO CubeSat program. ${ }^{22}$............. 8

1.8 University of Michigan GRIFEX CubeSat being prepared for P-Pod integration at Cal Poly SLO CubeSat clean room. ${ }^{38}$. . . . . . . 9

1.9 Friis ground station, one of three antennas at Cal Poly SLO. ${ }^{23} \ldots 10$

1.10 SpaceX Falcon Heavy test launch. ${ }^{4} \ldots \ldots$. . . . . . . . . . . 11

1.11 Thermal-vacuum chamber at Cal Poly SLO CubeSat facility. ${ }^{22}$. . 12

1.12 Impact hammer setup with an accelerometer on the test object (left side) and a load cell on the hammer tip (center) ${ }^{58}$. . . . . . . 13

1.13 Impact hammer measurement configurations. ${ }^{81} \ldots \ldots \ldots 14$

1.14 Slip table in horizontal and vertical configurations. ${ }^{77} \ldots \ldots$

1.15 Slip table vibration test in a horizontal configuration. ${ }^{22} \ldots \ldots$

2.1 Plot of a dynamic response before and after optimization. ${ }^{34} \ldots 18$

2.2 System with a resonance frequency of approximately $3.8 \mathrm{rad} / \mathrm{sec}^{.79} \quad 19$ 
2.3 Tacoma Narrows Bridge in 1940 undergoing, in part, wind resonance $(\text { left })^{5}$ prior to structural collapse (right) ${ }^{36} \ldots$. . . . . . . . . 20

2.4 Beats are a constructive and destructive sum of two different fre-

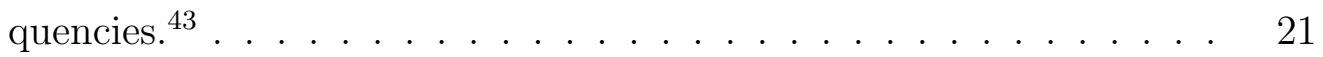

2.5 The amplitude of resonance continues to grow unbounded. ${ }^{46} \ldots 22$

2.6 Response plots with various damping conditions. ${ }^{80} \ldots$. . . . . 23

2.7 Exponential decay curve of an underdamped system. ${ }^{31} \ldots$. . . . 24

2.8 Half-power bandwidth method used to approximate the damping coefficient $\zeta$ of a vibration system. ${ }^{28}$. . . . . . . . . . 25

2.9 Block mass with 6 Degrees of Freedom (DOF). ${ }^{36} \ldots \ldots$. . . . . . 27

2.10 Simple element shapes used to discretize a body into finite elements. ${ }^{67} 29$

2.11 Example of a structure undergoing dynamic excitation. ${ }^{68} \ldots . . .30$

2.12 Cantilever bar composed of two elements. ${ }^{50} \ldots \ldots 32$

2.13 Modal analysis depicting the first four mode shapes of a cantilever ${\text { beam }{ }^{49} \ldots \ldots \ldots \ldots \ldots \ldots}_{33}$

3.1 Rendering of a $3 \mathrm{U}$ CubeSat design. ${ }^{2} \ldots \ldots . \ldots 34$

3.2 SolidWorks CAD model of the vibration table armature assembly. ${ }^{77} \quad 35$

3.3 3U Test Pod (left) and P-Pod (right) ${ }^{22}$ used to house CubeSats for dynamic testing. . . . . . . . . . . . . . . . 36

3.4 Floating plate and guide rails used to constrain CubeSats. . . . . . 37

3.5 Spring plungers (left) ${ }^{36}$ press onto the floating plate (right). . . . . 38

3.6 Removable cap of test pod. . . . . . . . . . . . . 38

3.7 Test pod (top) and the corresponding CAD model (bottom). . . . . 40

3.8 A $3 \mathrm{U}$ mass model (left) can be used as a substitute for CubeSats (right) $)^{23}$ in dynamic testing. . . . . . . . . . . . . . . . . . 41

3.9 CAD geometry of the mass model. . . . . . . . . . . . . 42

3.10 Fixture used to attach the test pod to the vibration table. . . . . . 43 
3.11 Bolt patterns on the bottom side of the fixture plate. . . . . . . . . 44

3.12 Fixture plate (top) and the corresponding CAD model (bottom). . 45

3.13 Loaded test pod (top) and the corresponding CAD assembly (bottom). 46

3.14 Cross-section of the CAD assembly showing the mass model is coincident with the inner cap wall (right side) of the test pod. . . . . . 47

3.15 Cross-section of the CAD assembly showing the mass model is coincident with the guide rails of the test pod. . . . . . . . . . . . . . 47

3.16 CAD assembly of the empty test pod with the fixture plate. . . . . 48

3.17 CAD assembly of the loaded test pod with the fixture plate. . . . . 48

3.18 CAD assembly (left) of a Cal Poly SLO PolySat structure with deployable solar panels ${ }^{70}$ (right). . . . . . . . . . . . . 49

3.19 Main components of the PolySat structure. . . . . . . . . . . 50

3.20 CAD assembly of the CubeSat CPX structure. . . . . . . . . . 51

3.21 Mounting panel allows various installation positions for a payload. . 52

3.22 CPX can accommodate various payload sizes and mounting configurations. . . . . . . . . . . . . . . 53

3.23 Single panels of CPX can be removed for easy access. . . . . . . . . 54

3.24 Slim-profile hinges sit away from the payload area. . . . . . . . 55

3.25 CPX battery pack is held together with four fasteners. . . . . . . 56

3.26 Battery pack slides in and bolts to one mounting panel. . . . . . . 56

3.27 CAD assembly of the test pod and CubeSat CPX structure. . . . . 57

3.28 Cross-section views of the test pod and CPX assembly. . . . . . . 58

3.29 CAD assembly of the loaded test pod with the fixture plate. . . . 58

4.1 Unholtz-Dickie Corp. vibration slip table (horizontal configuration). 59

4.2 Slip plate slides on a granite slab using oil lubrication to minimize friction and improve stiffness. . . . . . . . . . . . . . 60

4.3 Slip table control interface. . . . . . . . . . . . . . 61 
4.4 Tri-axial accelerometer measures the dynamic response. . . . . . . . 63

4.5 Basic internal structure of a typical accelerometer ${ }^{15}$. . . . . . . 64

4.6 Sensitivity of accelerometer using different mounting methods. ${ }^{52}$. 65

4.7 The test pod is bolted thru underside of the fixture plate. . . . . . 66

4.8 Small screws used for the test pod assembly. . . . . . . . . . . . . . 67

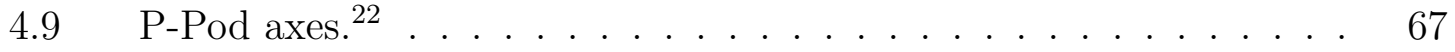

4.10 Bolted test pod assembly. . . . . . . . . . . . . . . . 67

4.11 Test pod cap is carefully replaced once the mass model has been loaded. . . . . . . . . . . . . . . . . . . 68

4.12 Spring plungers are adjusted once the mass model is loaded. . . . . 69

4.13 Large bolts used for the fixture assembly. . . . . . . . . . . . . . 70

4.14 Bolted test pod and mass model assembly. . . . . . . . . . . . . . 70

4.15 Triaxial (blue) and single-axis control (orange) accelerometers are attached to taped surfaces using an adhesive. . . . . . . . . . . . 71

4.16 Triaxial and control accelerometers verify the $0.5 \mathrm{~g}$ input. The reference pertains to the feedback from the vibration table controller. $\quad 73$

4.17 All fixture plate responses fall within NASA GEVS random vibration limits. . . . . . . . . . . . . . . . . . . . . . 74

4.18 Triaxial accelerometers on the beam and cap of the test pod. . . . 75

4.19 Test pod has as the largest response at approximately $550 \mathrm{~Hz}$, and control accelerometers verify the 0.5 g input. . . . . . . . . . 76

4.20 Random vibration of test pod has a peak response at $520 \mathrm{~Hz}$ and 1100 Hz. . . . . . . . . . . . . . . . . . . . . 77

4.21 Test pod response is similar to that of the pre sine sweep test, indicating no real changes occurred during the random vibration test. . 78

4.22 Triaxial accelerometers on the test pod and mass model. . . . . . . 79

4.23 The mass model has a more pronounced response than the test pod during the pre sine sweep test. . . . . . . . . . . . . . . 80 
4.24 Random vibration response of test pod and mass model. . . . . . .

4.25 Response of the test pod and mass model are similar to that of the pre sine sweep test, indicating no real changes occurred during the random vibration test. . . . . . . . . . . . . . .

4.26 Time-domain response of the empty test pod. . . . . . . . . . . . 84

4.27 Time-domain response of the loaded test pod. . . . . . . . . . . . 84

5.1 Adams View model of the vibration table setup. . . . . . . . . .

5.2 Adams model showing planar joints on the large base, and bushing connections at the test pod (yellow) and slip plate (red) interface. .

5.3 Adams model with output channels on the corner of the test pod (transparent) and side panel of the mass model. . . . . . . . . . .

5.4 Adams model with translational spring-damper connections between the test pod (transparent) and mass model. . . . . . . . . . . . . 88

5.5 Adams model of the test pod as a flexible body. . . . . . . . . . 88

5.6 Adams model of the test pod and fixture plate. . . . . . . . . . 89

5.7 Fixed joints are used to constrain the test pod to the fixture. . . . . 89

5.8 Output channels at the cap and beam of the test pod. A reference output is also located at the center of the fixture. . . . . . . . . . 90

$5.9 \quad 0.5 \mathrm{~g}$ input at the bottom surface of the fixture plate. . . . . . . 90

5.10 Adams model of the test pod assembly has a peak response near 600

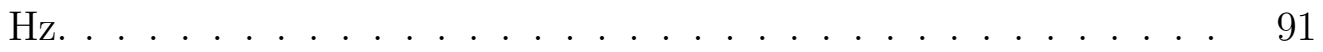

5.11 FEA model of a wrench and bolt with stress distributions. ${ }^{57} \ldots 92$

5.12 Test pod CAD (top), FEA partitions (middle), and wireframe view

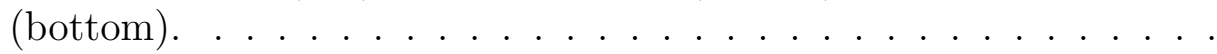

5.13 Small square partitions on the bottom panel of the test pod FEA model. . . . . . . . . . . . . . . . . 95

5.14 Mass model CAD (top) and FEA partitions (bottom). . . . . . . 96

5.15 Fixture plate CAD (top) and FEA partitions (bottom). . . . . 97 
5.16 FEA model of the empty test pod assembly (top), wireframe view (middle), and loaded test pod assembly (bottom). . . . . . . 98

5.17 Interaction between the mass model (purple) and test pod (red) surfaces of the FEA assembly. . . . . . . . . . . . . . . . . . 99

5.18 Tie constraint between the small partitions of the test pod (purple) and the top surface of fixture plate (red) . . . . . . . . . . 100

5.19 Tie constraint between the face of the mass model (purple) and the internal surface of the test pod cap (red) . . . . . . . . . 100

5.20 Various 3D element shapes that can be used in FEA models. ${ }^{11}$. . . 101

5.21 Linear vs Quadratic hexahedral elements. ${ }^{36} \ldots \ldots \ldots \ldots \ldots$

5.22 Various element types that can be used in FEA models. ${ }^{8} \ldots \ldots . .103$

5.23 Conventional vs Continuum (solid) shell elements available in Abaqus. ${ }^{9} 104$

5.24 Meshed FEA models of the test pod (top), mass model (middle), and fixture plate (bottom) . . . . . . . . . . . . . 105

5.25 Encastre boundary condition applied to the base of the FEA fixture plate. . . . . . . . . . . . . . . . . . 108

5.26 ANSYS model of the test pod with an auto-partitioned mesh. . . . 109

5.27 Meshed FEA assembly of the test pod and mass model. . . . . . . . 113

5.28 Simplified CPX structure (left) and mesh generation (right). . . . 114

5.29 Payload block is used to add rigidity to the CPX structure. . . . . 115

5.30 Meshed FEA assembly of the test pod and CubeSat CPX. . . . . . 116

5.31 Meshed FEA assembly of the test pod and CubeSat CPX (no payload).116

6.1 Example of an FEA mode shape for a 1U CubeSat frame. ${ }^{30} \ldots 117$

6.2 Natural frequencies of the empty test pod FEA assembly. . . . . . 118

6.3 Natural frequencies of the test pod with mass model FEA assembly. 120

6.4 Natural frequencies of the test pod with CPX FEA assembly. . . . 121

6.5 Natural frequencies of the test pod with CPX (no payload) FEA assembly. . . . . . . . . . . . . . . . . . 122 
6.6 General acceleration (GA) of Mode $2(665 \mathrm{~Hz})$ has the highest ASD response in the z-axis of the empty test pod FEA assembly. . . . . 123

6.7 Mode $2(664.96 \mathrm{~Hz})$ amplifies the displacement $(\mathrm{U})$ in the z-axis of the empty test pod FEA assembly. . . . . . . . . . . . . . . . 124

6.8 General acceleration (GA) of Mode $1(497 \mathrm{~Hz})$ has the highest ASD response in the z-axis of the test pod with mass model FEA assembly. 125

6.9 Mode $1(496.95 \mathrm{~Hz})$ amplifies the displacement $(\mathrm{U})$ in the z-axis of the test pod with mass model FEA assembly. . . . . . . . . . 126

6.10 General acceleration (GA) of Mode $2(517 \mathrm{~Hz})$ has the largest ASD response in the z-axis of the test pod with CPX FEA assembly. . . 127

6.11 Mode $2(516.85 \mathrm{~Hz})$ amplifies the displacement $(\mathrm{U})$ in the z-axis of the test pod and CPX FEA assembly. . . . . . . . . . . 128

6.12 General acceleration (GA) of Mode $11(549 \mathrm{~Hz})$ has the largest ASD response in the z-axis of the test pod with CPX (no payload) FEA assembly. . . . . . . . . . . . . . . . . . 129

6.13 Mode $11(548.16 \mathrm{~Hz})$ amplifies the displacement (U) in the z-axis of the test pod and CPX (no payload) FEA assembly. . . . . . . . 130

6.14 Stress areas of the empty test pod under a $1 \mathrm{~N}$ lateral load. . . . . 132

6.15 Underdamped response of the empty test pod FEA assembly. . . . 133

6.16 Stresses at the six screw locations and overhang areas of the empty test pod assembly. . . . . . . . . . . . . . . 133

6.17 Stress areas of the test pod with mass model FEA assembly. . . . . 134

6.18 Underdamped response overlay of the test pod with mass model FEA assembly. . . . . . . . . . . . . . . . 135

6.19 The screws closest to the applied load see the highest stress levels in the test pod with mass model FEA assembly. . . . . . . . . . . . 135

6.20 Underdamped response overlay of the empty and loaded test pod FEA assembly. . . . . . . . . . . . . . . 136

6.21 Stresses of the test pod and CPX assembly under a $1 \mathrm{~N}$ load. . . . 137

6.22 Transient response overlay of the test pod and CPX assembly. . . . 138

6.23 Stresses at the six screw locations of the test pod and CPX assembly. 138 
6.24 Notable peaks from the sine sweep empty test pod data. . . . . . . 139

6.25 Prominent ASD peaks are labeled in the experimental data of the empty test pod. . . . . . . . . . . . . . . 141

6.26 ASD peaks (first seven modes) of the FEA empty test pod model (reprinted for comparison). . . . . . . . . . . . . . . . 141

6.27 Notable peaks from the sine sweep loaded test pod data. . . . . . . 143

6.28 Prominent ASD peaks are labeled in the experimental data of the loaded test pod. . . . . . . . . . . . . . . . . 145

6.29 ASD peaks (first nine modes) of the FEA loaded test pod model (reprinted for comparison) . . . . . . . . . . . . . . . 145

6.30 PolySat structure ${ }^{23}$ (left) and CubeSat CPX (right). . . . . . . . . . 147

6.31 Notable peaks in the sine sweep data of the PolySat structure. . . . 148

6.32 Notable peaks in the random vibration data of the PolySat structure. 149

6.33 ASD peaks (first nine modes) of the test pod with CPX FEA assembly (reprinted for comparison). . . . . . . . . . . . . . 150

7.1 Test pod (left), mass model (center), and CPX assemblies (right) evaluated in this work. . . . . . . . . . . . . . . . . . 152

7.2 CubeSat circuit boards under vibration loads are susceptible to bending and losing components due to poor soldering. ${ }^{63}$. . . . . . . 153

7.3 Optional fixture plate that can be used with the test pod. Note that the test pod base is fully supported by this fixture plate. ${ }^{22}$. . . . 154

7.4 CubeSat and P-Pod prior to integration. ${ }^{27} \ldots \ldots \ldots$ 
Chapter 1

\section{INTRODUCTION}

\subsection{Background and Scope}

Satellites used in space exploration withstand harsh conditions including large temperature gradients, intense UV radiation, impact of space debris, and the effects of a vacuum environment. As such, they are designed to function under such conditions and with an intended lifespan that will be sufficient to complete the objectives of the mission. However, challenges to maintain proper operation begin as early as the launch phase of the satellite, where violent dynamic forces known as vibration are transmitted from the rocket as it propels its way thru the Earth's atmosphere and into outer orbit (see Figure 1.1). So, satellites must be designed to first withstand these strong vibration conditions prior to their release into orbit.

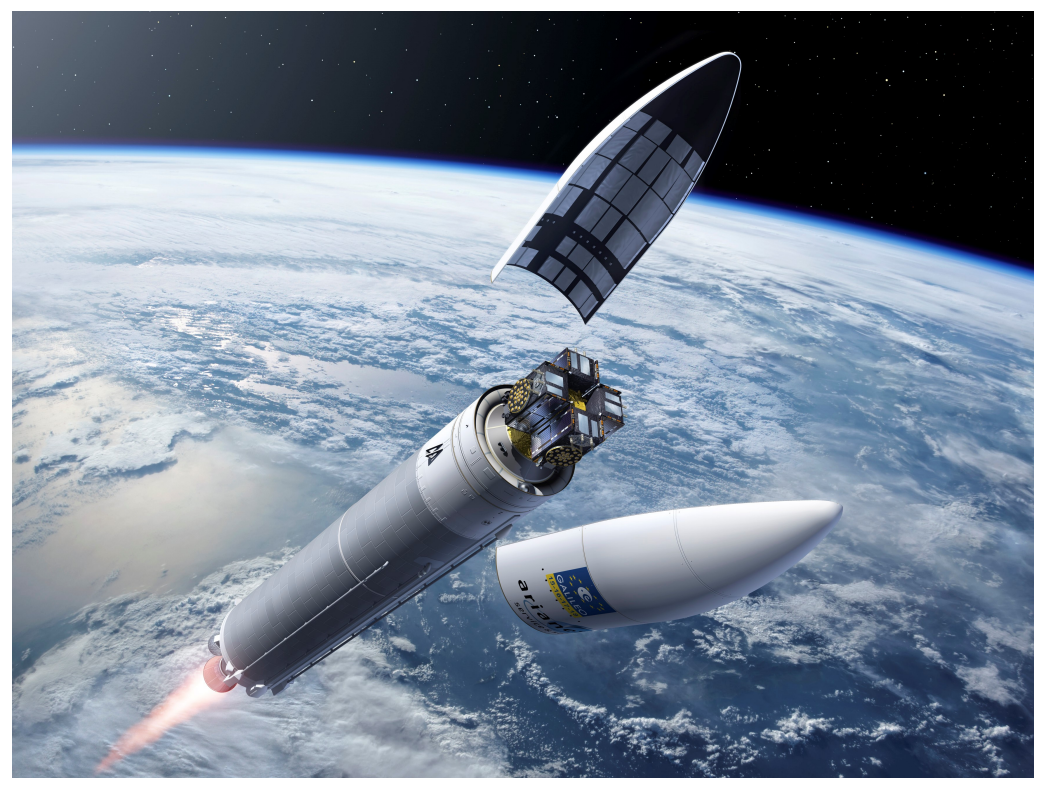

Figure 1.1: Satellites experience strong vibration forces during the launch phase of a rocket. 39 
CubeSats are small satellites (nanosatellites) which are used as a more cost-effective option for space missions (see Figure 1.2). Like their larger counterparts, CubeSats must be designed to withstand both launch and space conditions to ensure proper functionality throughout their intended life time in space.

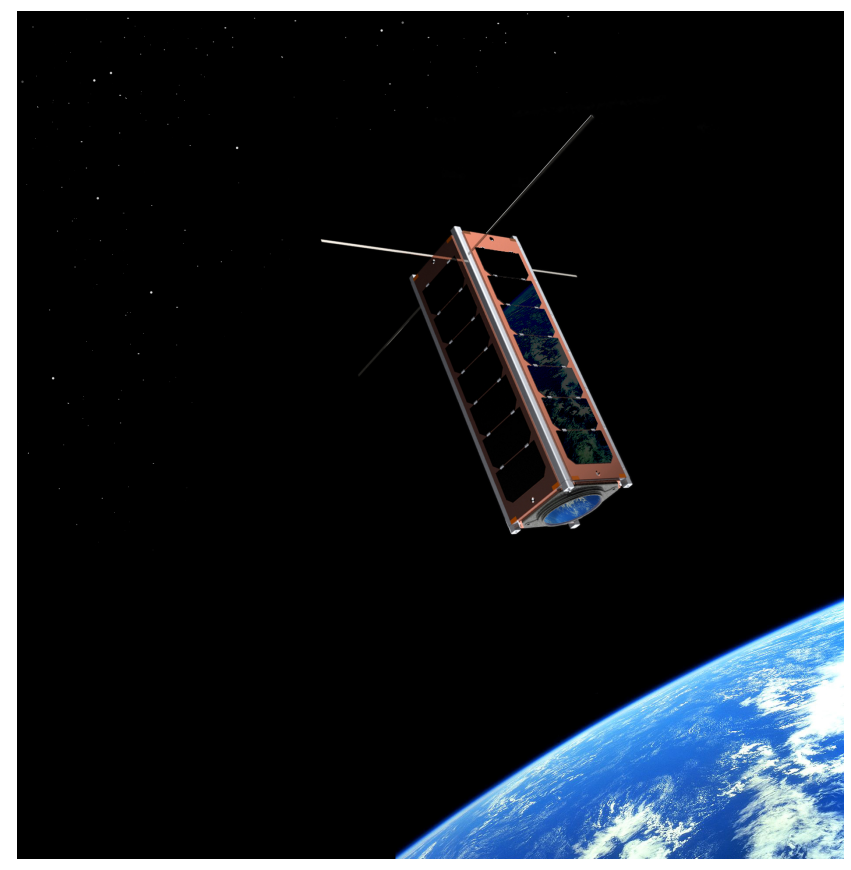

Figure 1.2: $3 \mathrm{U}$ CubeSat in orbit. ${ }^{74}$

In particular, unforeseen vibration can have detrimental effects on a satellite, ranging from structural to electrical system failure. Therefore, satellites undergo rigorous testing to check that they can survive various levels of vibration, and CubeSats are no exception. However, one of the main challenges of analyzing vibration in CubeSats (and satellites in general) is the difficulty of replicating rocket launch conditions in a lab setting. Since CubeSats are integrated with other components and sub-assemblies of the launch vehicle, all the different interactions affect the dynamics of vibration in a way that is unique to that particular launch. Fortunately, design guidelines have been established for CubeSats using data collected from past missions, but allow room for optimization. 
As all testing of CubeSats occurs in a lab setting, the results obtained from these test runs does not always reflect the true dynamics of a real launch phase. While vibration tests in a lab may provide sufficient evidence to qualify a CubeSat for launch, the design can only be truly evaluated after the vibration test is complete. As such, troubleshooting and re-testing can be time consuming, and iterating designs can be expensive due to the long lead times necessary for manufacturing and assembly of CubeSats.

The purpose of the work presented here was to use finite element analysis (FEA) to develop a computer model that can help predict the dynamic behavior of CubeSats early in the design phase, and identify possible design flaws so corrections can be made prior to manufacturing and assembly. Specifically, natural frequencies were the main focus of the analysis, as they play a key role in the vibration response of CubeSats. The model created here is based on a vibration test setup used by the CubeSat program at Cal Poly San Luis Obispo, and is meant to serve as a stepping stone in further understanding of how vibration behavior is influenced by not only the internal components of the CubeSat, but also by the surrounding constituents. The FEA model was validated using experimental data from the vibrations test setup, and then used to evaluate a concept CubeSat structure to see the application of the model. Again, while this work was used to simulate results observed in a lab setting, the ultimate goal is to build upon this model in future works and aim towards better understanding of how CubeSats truly behave when integrated to actual launch vehicles.

The following sections of this chapter are brief introductions about the inception of CubeSats, their design, and the organizations that help make their space ventures possible. 


\subsection{CubeSat Program}

The CubeSat standard started as a joint effort between California Polytechnic State University San Luis Obispo (Cal Poly SLO) and Stanford University to provide students more accessible and affordable space launch opportunities. Since its inception in 1999, universities, government organizations, and private firms around the world have adopted the standard in efforts to develop the next generation of CubeSats. ${ }^{22}$

Two programs, CubeSat and PolySat (see Figure 1.3), are based in California Polytechnic State University San Luis Obispo, where both programs function under the CubeSat lab umbrella. Composed of students and faculty from different disciplines, the CubeSat program is responsible for testing and integration of CubeSats and serve as the gateway between CubeSat developers and launch providers around the world. The CubeSat program ensures that CubeSats meet both design standards and requirements of a given launch provider.
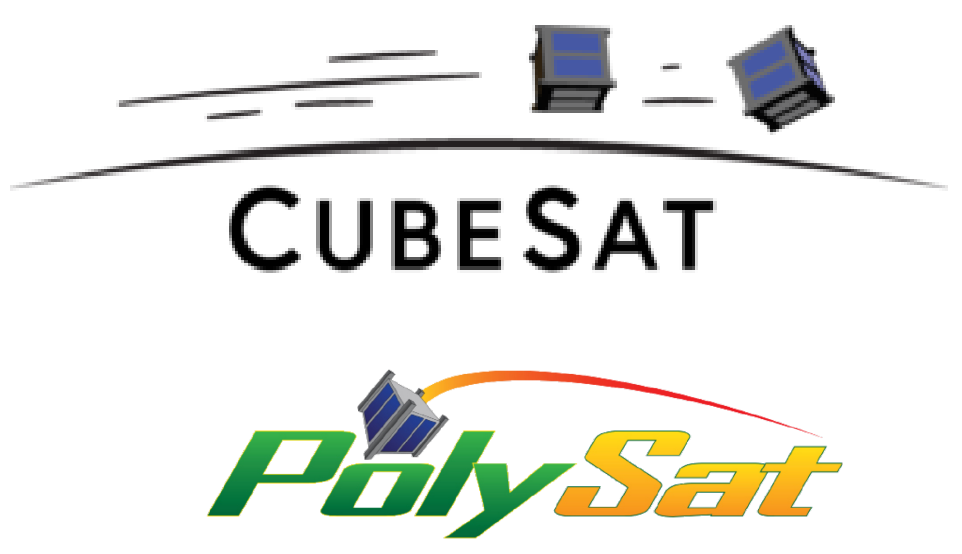

Figure 1.3: CubeSat ${ }^{22}$ and PolySat ${ }^{23}$ programs based in Cal Poly San Luis Obispo, California. 
Like the CubeSat program, PolySat is also a multi-disciplinary program composed of students and faculty that designs, manufactures, and builds CubeSats in house. Teams work together with sponsors that seek to develop a CubeSat that meets the needs of their payload. Both CubeSat and PolySat programs have collaborated in many missions and are responsible for successful CubeSat launches including CP10 (ExoCube) and DAVE (Damping And Vibrations Experiment) which are currently in orbit $^{23}$ as of the publishing date of this paper.

\subsection{ELaNa}

Based out of the Kennedy Space Center in Florida and managed by the Launch Services Program (LSP), Educational Launch of Nanosatellites (ELaNa) is a program developed by NASA that reaches out to students with the purpose of generating interest in the fields of science, technology, engineering, and mathematics (STEM). ${ }^{12}$ The ELaNa program allocates missions to academic institutions including colleges and high schools throughout the United States, allowing students of multiple disciplines to engage in the development of CubeSats from early design to launch vehicle integration. CP5 (Cal Poly SLO) for example launched as part of the ELaNa-6 mission back in

2007, serving as a de-orbiting experiment using a deployed thin-film mechanism. ${ }^{23}$ 


\subsection{CubeSat Design Standard}

The CubeSat standard includes design and test requirements that must be met before a CubeSat is cleared for launch vehicle integration. A $1 \mathrm{U}$ CubeSat for example (see Figure 1.4) requires that the mass be less than $1.33 \mathrm{~kg}$, have a width of $100.0 \mathrm{~mm}$, and a height of $113.5 \mathrm{~mm} .{ }^{22}$ Each CubeSat design carries its own set of requirements, all of which can be found on the CubeSat Design Specification document provided on the Cal Poly SLO CubeSat website. ${ }^{22}$ The design of a CubeSat depends on the application and size of the payload that is to be integrated (see Figure 1.5). CubeSats serve as the housing and "life support" of a given payload, providing protection during the violent launch phase and sustenance while the satellite operates in the harsh environment of Earth's orbit.

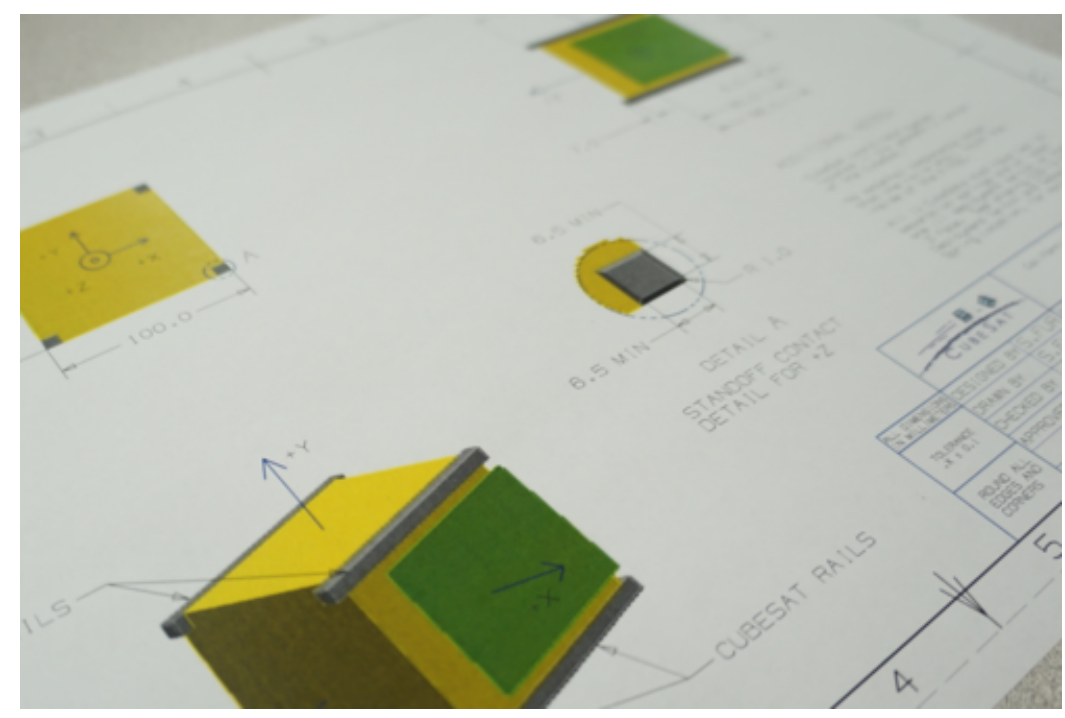

Figure 1.4: Snapshot of 1U CubeSat drawing. ${ }^{22}$ 


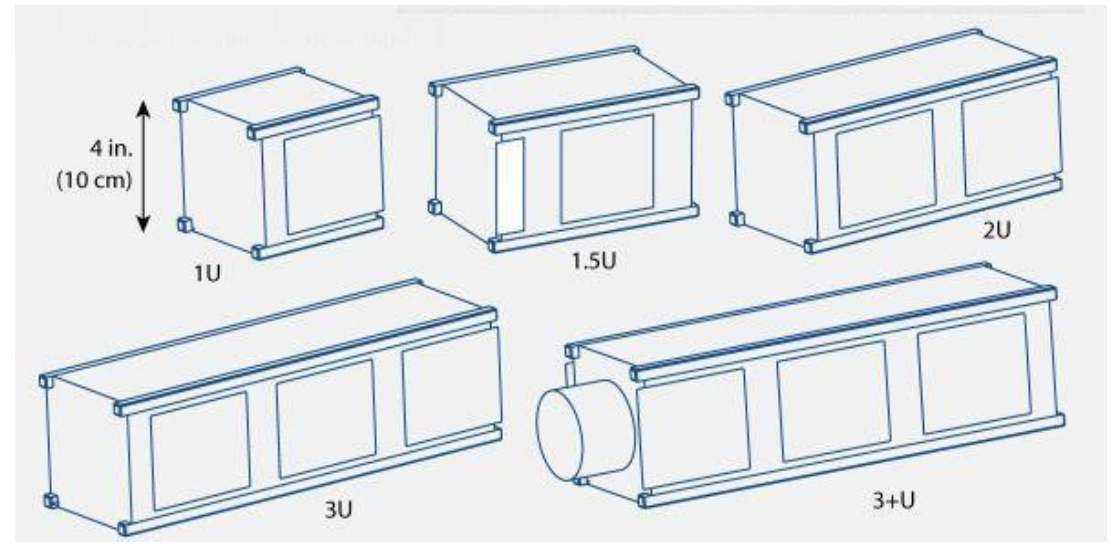

Figure 1.5: Size comparison of various CubeSat design options. ${ }^{69}$

CubeSats have built-in battery packs which provide power to the system upon receiving command. Solar cells may also be integrated into the CubeSat design to help supplement the power needs of the CubeSat and its enclosed payload (see Figure 1.6). Furthermore, current efforts include adding efficient propulsion capabilities to CubeSats which would allow them to venture further out into deep space. This is a challenging endeavour due to the relatively small size and limited available space of CubeSats. The compact propulsion system itself presents an added level of complexity to the design of a CubeSat, as it must not compromise the operation of the other systems in the CubeSat.

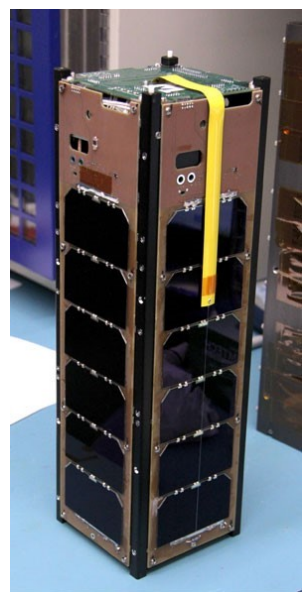

Figure 1.6: GRIFEX CubeSat developed by the University of Michigan with NASA ESTO and JPL. ${ }^{38}$ 


\subsection{Poly Picosatellite Orbital Deployer}

The Poly Picosatellite Orbital Deployer (P-Pod) serves as the interface between the CubeSat and the launch vehicle (see Figure 1.7). Just as a CubeSat serves as the housing for a payload, the P-Pod houses and protects the CubeSat during the launch phase. With a similar function to a "jack-in-the-box," the P-Pod uses a large spring and a set of rails to guide and eject the CubeSat upon a signal command. The P-Pod lid is held closed during launch by a release mechanism that can only be triggered once the launch vehicle systems determine suitable conditions for ejection. If suitable conditions are met, the lock is triggered, releasing the CubeSat into space and allowing it to start communications with ground stations on Earth.
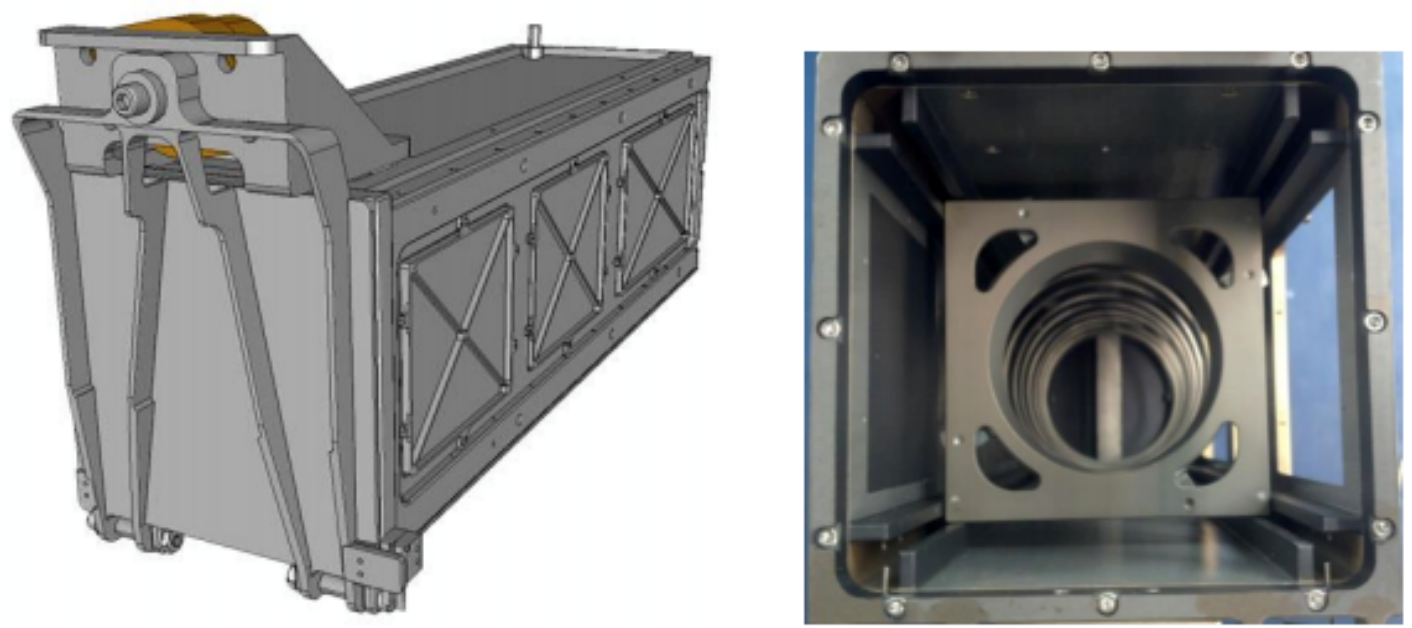

Figure 1.7: Poly Picosatellite Orbital Deployer (P-Pod) developed by the Cal Poly SLO CubeSat program. ${ }^{22}$

Like CubeSats, P-Pod's adhere to strict design requirements that must also be tested and validated in order to properly function after withstanding launch phase conditions and the extreme environment of space. Compatibility is one of the main reasons why CubeSats must follow and meet design specifications, as this will ensure proper fit during assembly and a successful ejection once in space (see Figure 1.8). 
Above all is the safety of the launch vehicle itself. It should be noted that CubeSats are usually not the main payload of a flight mission, relying instead on finding launch providers that can allocate unused space on their launch vehicle. As main payloads can be much larger, more complex, and significantly more expensive than CubeSats, great care must be taken that secondary payloads (like CubeSats) will not jeopardize the safety of the launch vehicle and main payload, or interfere with the main objectives of the launch vehicle mission. Such occurrences would not only be detrimental to the mission, but would also put the entire CubeSat program at risk including limited access to launch providers or being banned from future missions entirely.

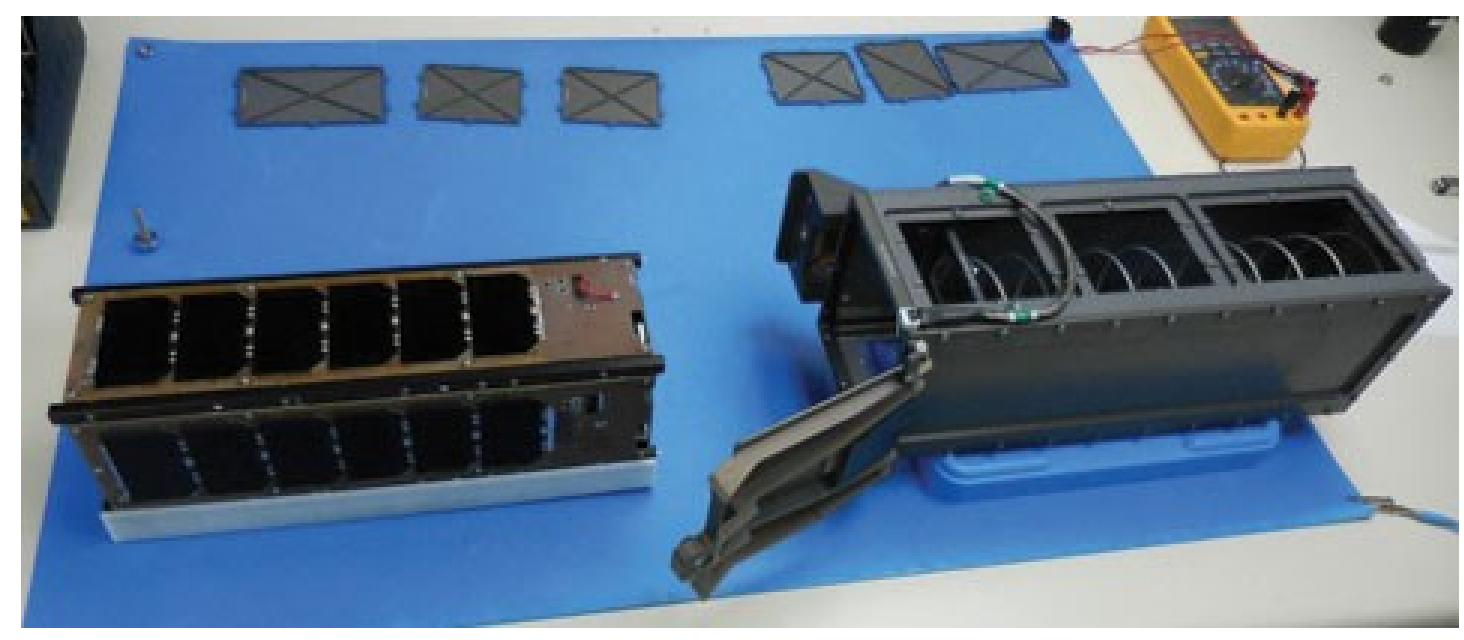

Figure 1.8: University of Michigan GRIFEX CubeSat being prepared for P-Pod integration at Cal Poly SLO CubeSat clean room. ${ }^{38}$ 


\subsection{Ground Stations}

Ground stations exist around the globe and serve as the command center of CubeSats, tracking their position in orbit and system health overall. Data exchange and communication takes place at these stations, and they can be configured to meet the needs of a given mission. Cal Poly SLO is home to three such stations including Hertz, Marconi, and Friis (see Figure 1.9). The Hertz station was the first ground station built at Cal Poly in order to support the CP1 CubeSat (Cal Poly SLO), followed by Marconi which was built to support the CP2 CubeSat (Cal Poly SLO). ${ }^{23}$ Both CP1 and CP2 were part of the same launch mission, but unfortunately never made it to orbit due to a launch failure. However, both of these stations went on to support the successful launch of CP3 (Cal Poly SLO) and CP4 (Cal Poly SLO) in a following mission. The Friis station is the latest member of the Cal Poly SLO ground stations, built not only to support future missions but also having the ability to experiment with higher link margins and data rates. ${ }^{23}$

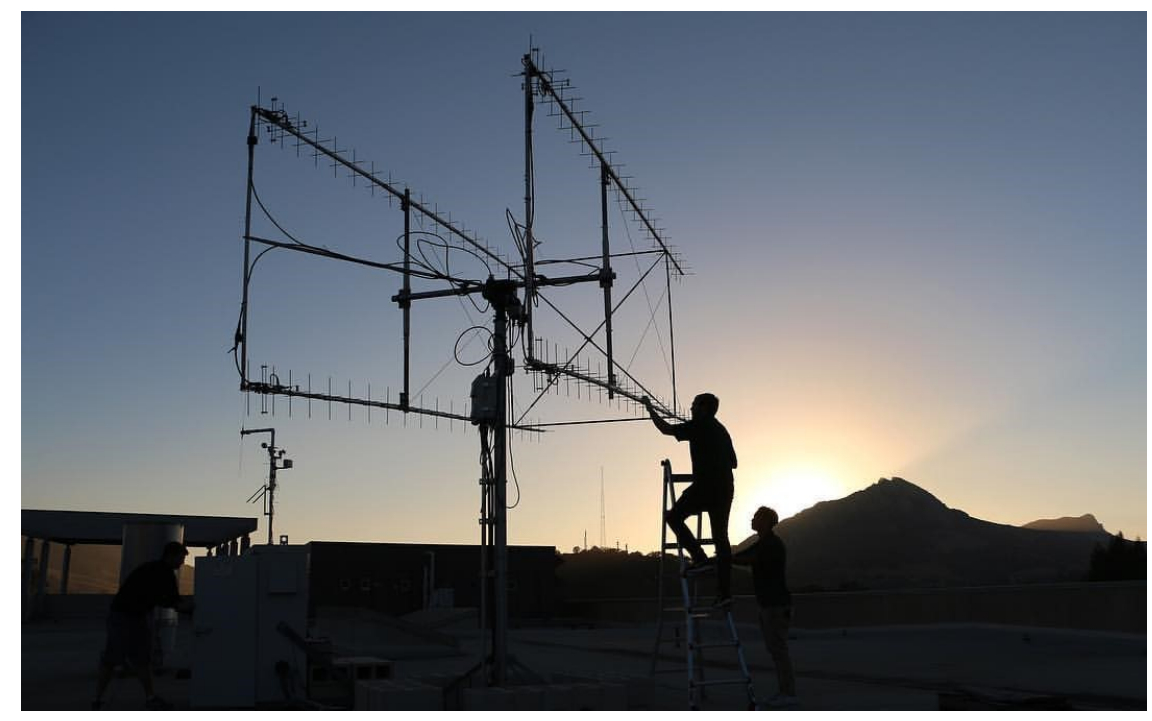

Figure 1.9: Friis ground station, one of three antennas at Cal Poly SLO. ${ }^{23}$ 


\subsection{Launch Environment and Testing}

During the launch phase, a spacecraft withstands the rigors of intense forces due to a combination of factors including acoustics, vibrations, and thermal loading. ${ }^{48}$ Sources of these forces include propulsion systems (see Figure 1.10) and the interaction between structural components, and as such, these forces can then be transferred to internal systems of the launch vehicle, including the payloads. For this reason, it is important that payloads, like CubeSats, undergo rigorous testing beforehand to ensure proper function after the launch phase has ended and forces have subsided.

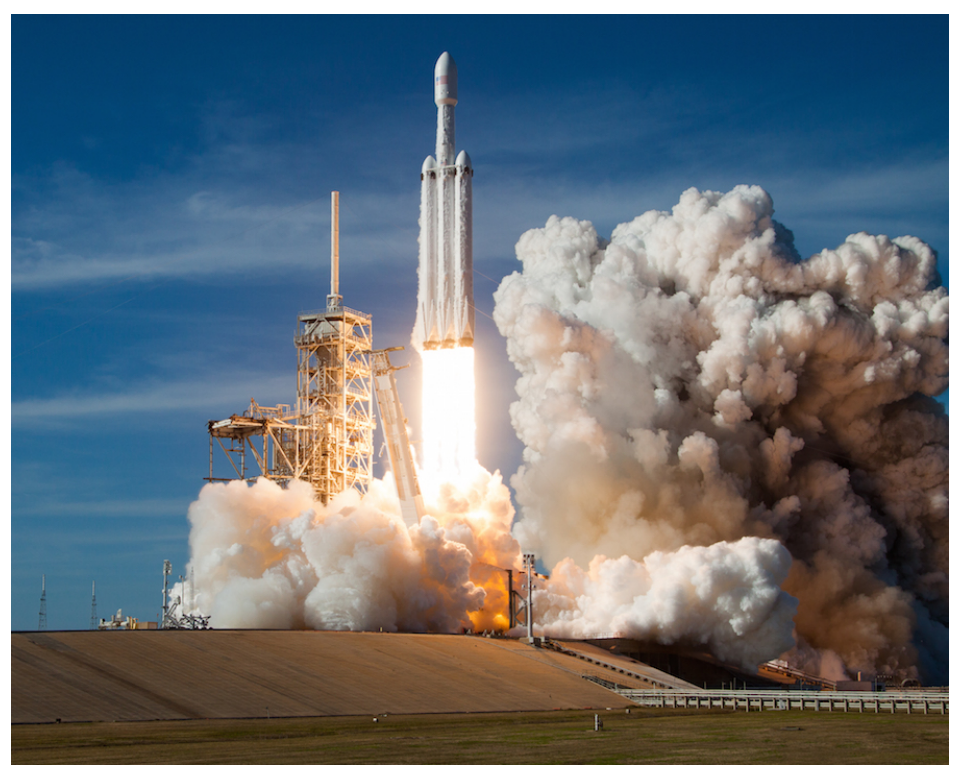

\section{Figure 1.10: SpaceX Falcon Heavy test launch. ${ }^{4}$}

CubeSats undergo thermal testing, where the nanosatellite is subject to a thermal cycling profile within a vacuum chamber in order to mimic temperature gradients in space (see Figure 1.11). Thermocouples are mounted to different sections of the CubeSat and then routed to a data acquisition system (DAQ) outside of the chamber. A technician monitors the chamber activity on a computer screen and records any anomalies that may occur during testing, such as the effects of trapped gas in a 
material, otherwise known as outgassing. Some tests may also include activation of the CubeSat in order to inspect system functionality and ensure it can operate in space as intended. If unforeseen issues arise, the CubeSat is removed from the chamber, troubleshooted, and retested as necessary.

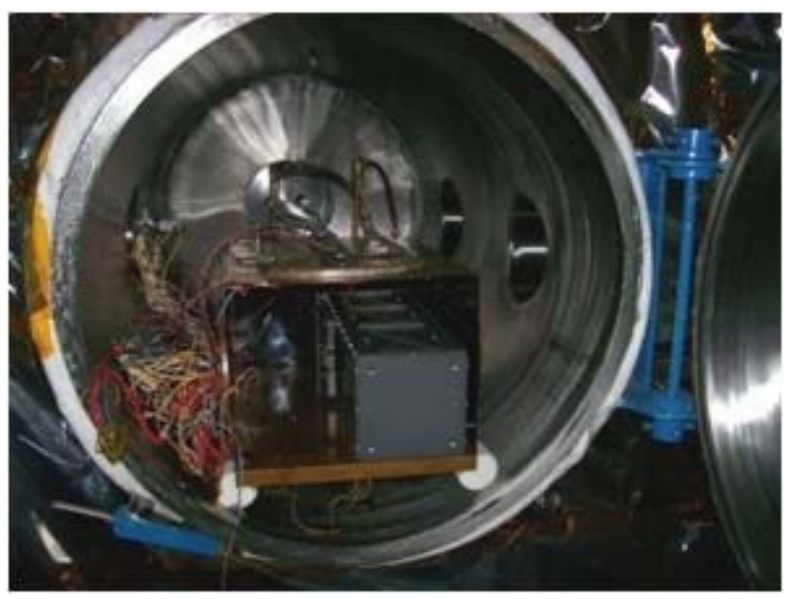

Figure 1.11: Thermal-vacuum chamber at Cal Poly SLO CubeSat facility. ${ }^{22}$

Vibration tests are performed to inspect for loose components and overall integrity of the CubeSat structure. Random vibration tests mimic the vibration dynamics of the launch phase, while sine sweep tests help identify the natural frequencies of the structure. These tests help determine if the excitement of such frequencies during launch would have destructive effects (resonance) on the structure or its internal components, like the payload. Although CubeSats are fairly rigid assemblies, each component of the assembly may respond differently to a given frequency, so it is important that a range of frequencies be tested to ensure system functions remain uncompromised after the launch phase.

It is difficult to replicate rocket launch dynamic conditions in a lab setting, but instruments are available to help mimic characteristics of such conditions. Impact hammers for example (see Figure 1.12) use an impulse force over a short time duration to excite specific modes of vibration that are proportional to the contact time and 
force of the hammer strike. ${ }^{81}$ This in turn identifies the frequency that is excited at such a mode, just as launch vehicle dynamics excite these modes during the launch phase. Different methods of measurement can be applied to impact hammer tests, each having their own level of accuracy and time required to complete the test (see Figure 1.13). Choosing the best measurement method will depend on the object being tested, its application, and the level of accuracy that is needed. Benefits of using impact hammers include their small size, portability, short testing duration, and how inexpensive they can be compared to other methods of identifying natural frequencies. However, this type of testing does have its drawbacks, as it is limited to relatively small objects due to the increased effects of signal noise which becomes more apparent with larger objects. ${ }^{81}$ This type of noise can interfere with measurement readings, yielding inaccurate data results.

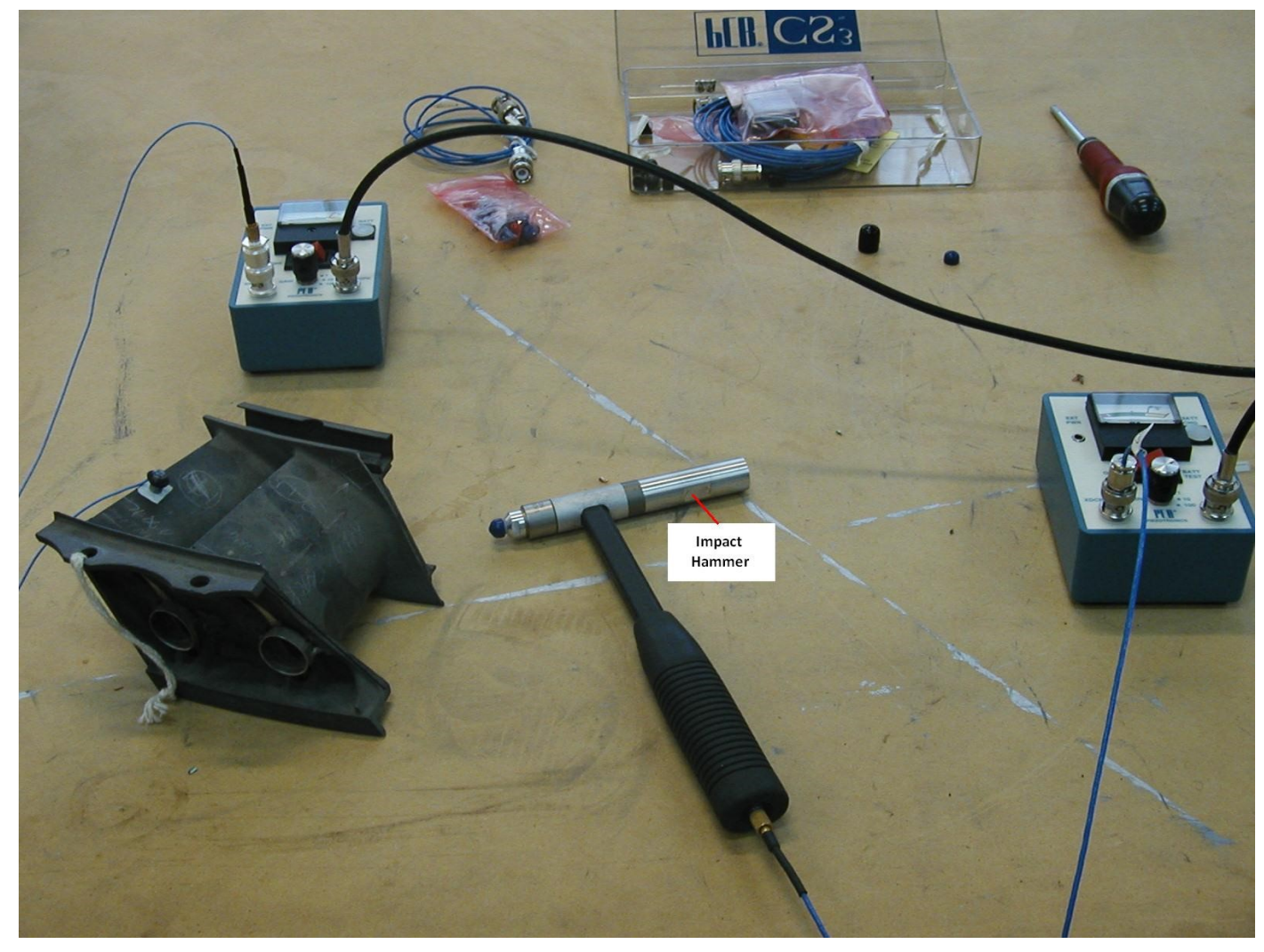

Figure 1.12: Impact hammer setup with an accelerometer on the test object (left side) and a load cell on the hammer tip (center). ${ }^{58}$ 

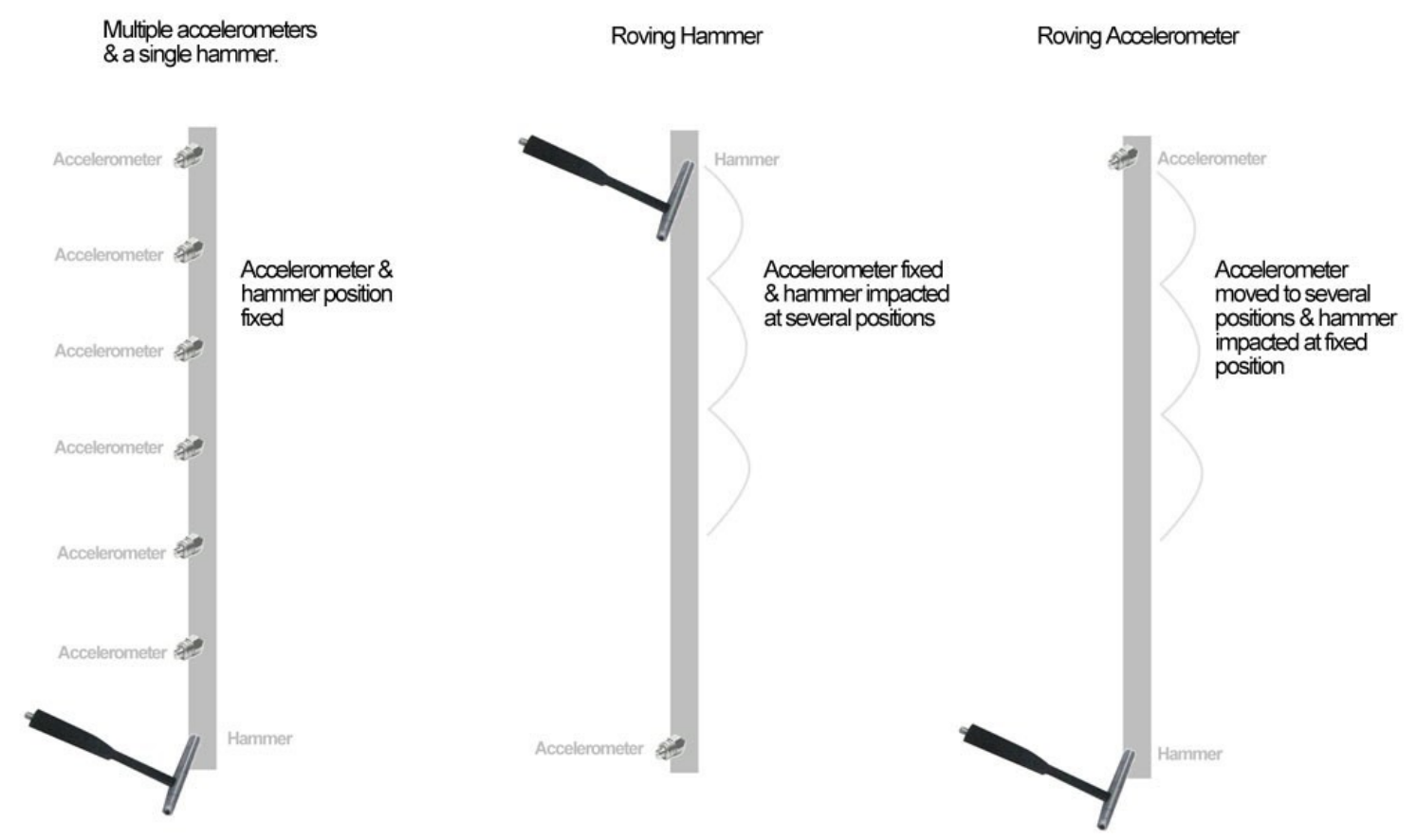

Figure 1.13: Impact hammer measurement configurations. ${ }^{81}$

Slip tables, also known as shake tables, are another type of instrument used to perform vibration tests. These type of shakers are electro-dynamic, using electricity to drive an armature along a single axis. Some models can be configured to vertical or horizontal positions, depending on the type of testing required (see Figure 1.14). In a horizontal configuration for example, the armature is bolted to a magnesium slip plate which rests on a slab of granite for added stiffness. ${ }^{77}$ Oil is fed to the slip plate and granite slab interface in order to minimize friction, allowing the slip plate to slide "freely" over the granite slab. 


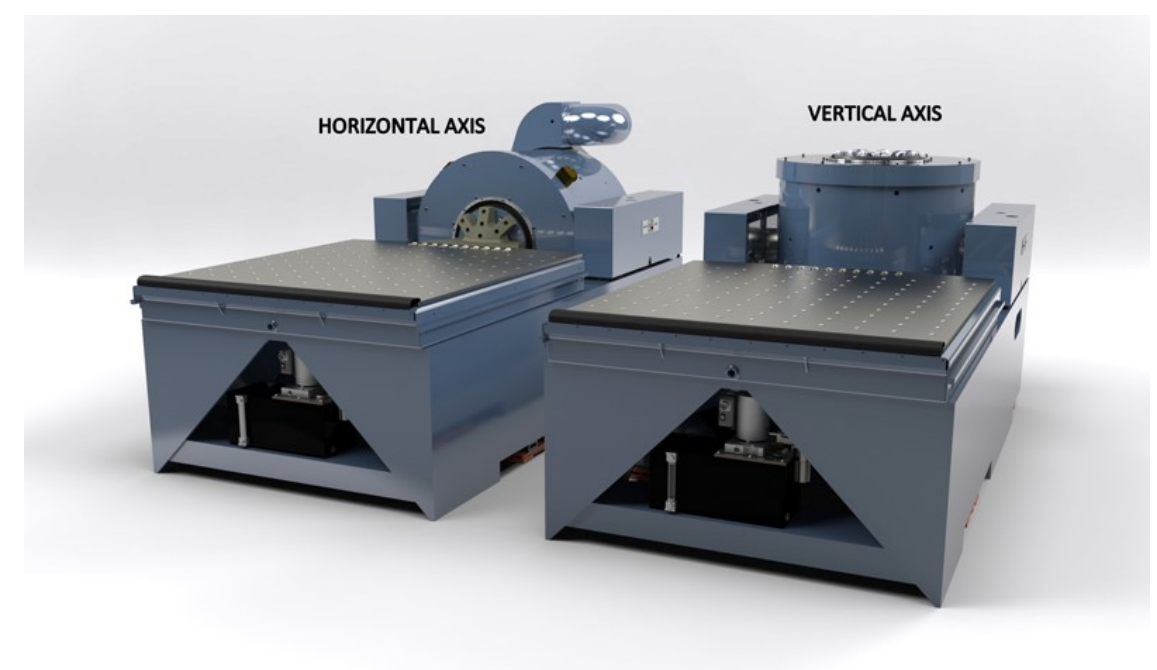

Figure 1.14: Slip table in horizontal and vertical configurations. ${ }^{77}$

To run vibration tests using a slip table, the object is bolted to the slip plate (horizontal configuration), or directly to the moving armature (vertical configuration). The desired test is programmed into the slip table controller system as an input profile, and accelerometers attached to the test object monitor the dynamic response during testing (see Figure 1.15). Like impact hammers, sudden "spikes" in the dynamic response plot signal the presence of natural frequencies.

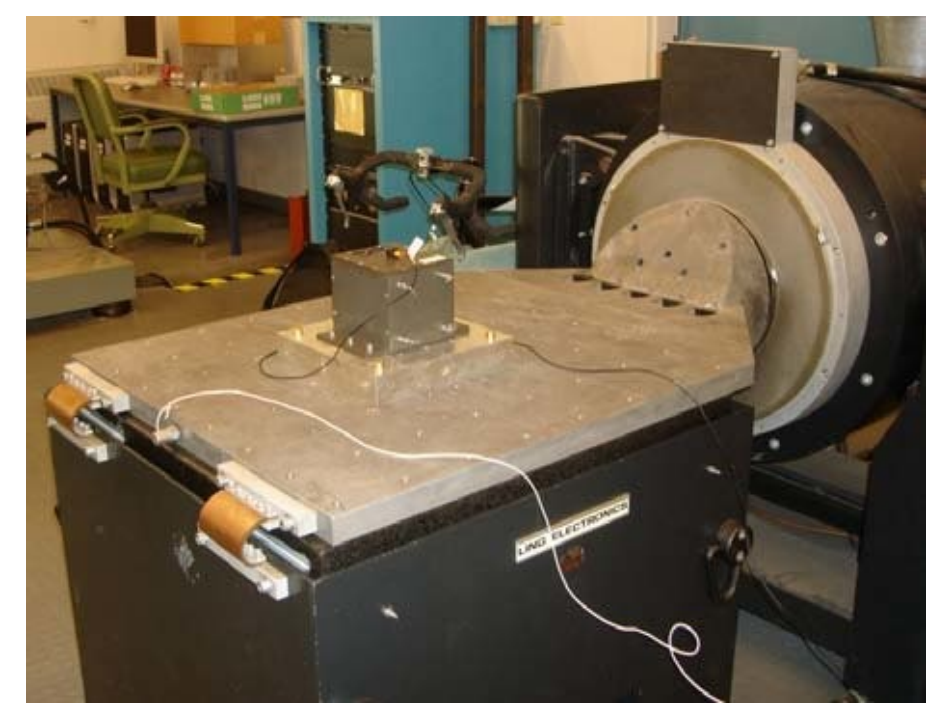

Figure 1.15: Slip table vibration test in a horizontal configuration. ${ }^{22}$ 
Shakers are available in a variety of sizes, some small enough to fit in the palm of a hand, to others with platforms large enough to shake entire buildings. This versatility is one of the appeals of using slip tables, and their robust design adds rigidity to help minimize the effects of pitching and torsional vibrations that can distort the accuracy of the system response. ${ }^{77}$ Disadvantages of slip tables however, may include their relatively high cost, long setup time, and bulky design which makes relocation difficult.

\subsection{NASA GEVS}

On May 1, 1959, NASA established the Goddard Space Flight Center (GSFC) to serve as the hub of mission control communications. ${ }^{14}$ The GSFC is responsible for carrying out NASA's space missions, and as such, testing procedures are held to high standards to ensure spacecrafts and equipment can survive and operate in the harsh environment of space. In 1969, the GSFC established the General Environmental Verification Specification (GEVS), a baseline of environmental testing standards that

continue to be updated and followed to this day. ${ }^{13}$ GEVS establishes performance requirements for various tests including structural loading, vibroacoustics, shock, mechanical function, and thermal behavior, ${ }^{13}$ with the level of testing depending on mission objectives and the type of equipment to be used.

CubeSats fall under the jurisdiction of GEVS requirements, and must undergo testing that meet or exceed these requirements before they can be qualified for launch vehicle integration. In general, an engineering test unit (ETU) is first manufactured as a prototype and subject to preliminary testing like shaker vibration. If the ETU tests are successful, a second unit is manufactured (flight unit) which will move on to additional testing and launch vehicle integration. 
Chapter 2

\section{THEORETICAL ANALYSIS}

\subsection{Nature of Vibration}

"Vibration is the subdiscipline of dynamics that deals with repetitive motion," 84 and it so happens that this type of motion exists everywhere in nature including mechanical, structural, and even biological systems. Whether it be structures swaying back and forth in the wind, or the simple pluck of a guitar string, vibrations play important roles in day-to-day life. While some types of vibration are of benefit, other types of vibration can be troublesome, if not destructive. It is for these reasons that great efforts go into analyzing this type of motion in order to understand the motion behavior and mitigate any negative effects that may arise from it. Engineers and scientists work together to find solutions to problems caused by vibrations, and although some types of vibration are impossible to eliminate completely, solutions can be developed to help remedy the effects. Buildings for example are retrofitted for added support during an earthquake, and vehicles make use of a spring-damper system connected to each wheel in order to protect the vehicle and passengers from excessive road vibration.

Like any other structure that is a exposed to a vibration environment, CubeSats are prone to damage and failure if not designed for such conditions. As previously mentioned, outgassing is a problem when attempting to use materials that can cushion and protect the satellite from vibration forces. Current designs rely on "hard" contacts between the CubeSat and their adjacent components, and this allows large forces to be transmitted from the launch vehicle to the CubeSat. So, it is essen- 
tial to understand if and how these transmitted forces affect the CubeSat and its performance.

Although the use of damping materials in the vacuum of space is limited, there are other factors that can help minimize the effects vibration, including the mass and stiffness of the CubeSat assembly. The mass of a CubeSat is not only influenced by the material used, but also by the design of the structure (e.g. solid vs hollow). A change in mass of a CubeSat affects its inertia, which resists motion induced by external forces like those from a rocket. The stiffness of a CubeSat also depends on the material and design of the structure, which helps resist the bending moments induced by external vibration. Furthermore, the assembly itself and the joint methods used (e.g. fastener vs adhesive) also affects the stiffness of the CubeSat system.

From the previous discussion, it is clear that different structure designs will have a different vibration response. The design of a CubeSat structure is imperative not only for self integrity, but to also provide adequate protection for the delicate equipment that it houses. Vibration analysis is necessary in order to optimize the dynamic performance of CubeSats and avoid that the natural frequencies of the structure be amplified by the vibration levels of a launch vehicle (see Figure 2.1).

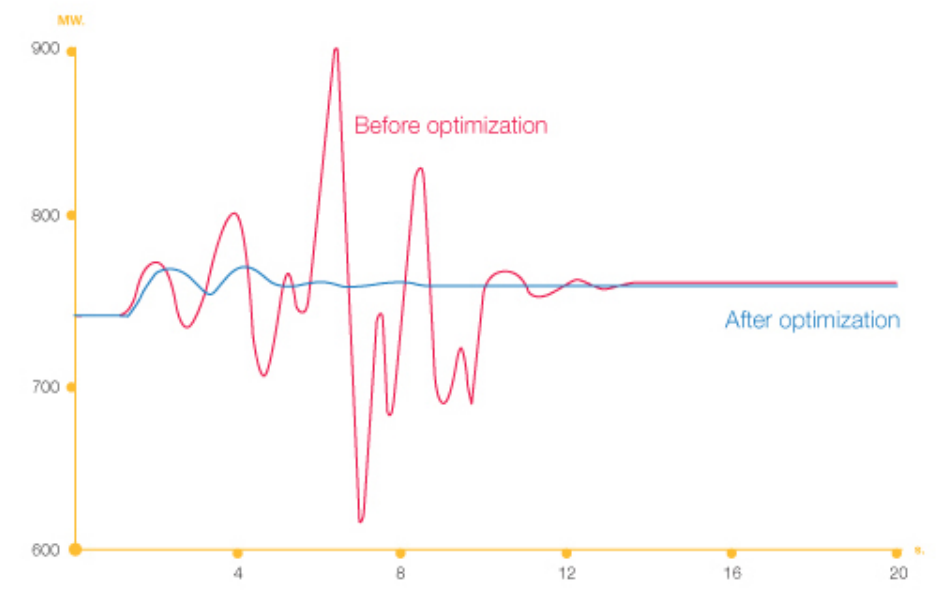

Figure 2.1: Plot of a dynamic response before and after optimization. ${ }^{34}$ 


\subsubsection{Resonance}

The general solution $x(t)$ for an undamped spring-mass system under harmonic excitation is defined as

$$
x(t)=\frac{v_{0}}{\omega_{n}} \sin \omega_{n} t+\left(x_{0}-\frac{f_{0}}{\omega_{n}^{2}-\omega^{2}}\right) \cos \omega_{n} t+\frac{f_{0}}{\omega_{n}^{2}-\omega^{2}} \cos \omega t
$$

where $v_{0}$ and $x_{0}$ are the initial conditions, $\omega_{n}$ is the natural frequency, $f_{0}$ is the massnormalized force, and $\omega$ is the driving frequency of the applied force (see Appendix A.5 for more details). In this equation, the term $\omega_{n}{ }^{2}-\omega^{2}$ is of significant importance for vibrating systems. Note that as the driving frequency $\omega$ approaches the natural frequency $\omega_{n}$ of the system, the denominator value becomes very small, resulting in a substantial increase in the amplitude value of the system response. At these points, large "peaks" mark the sudden amplification of the response (see Figure 2.2), a phenomena known as resonance.

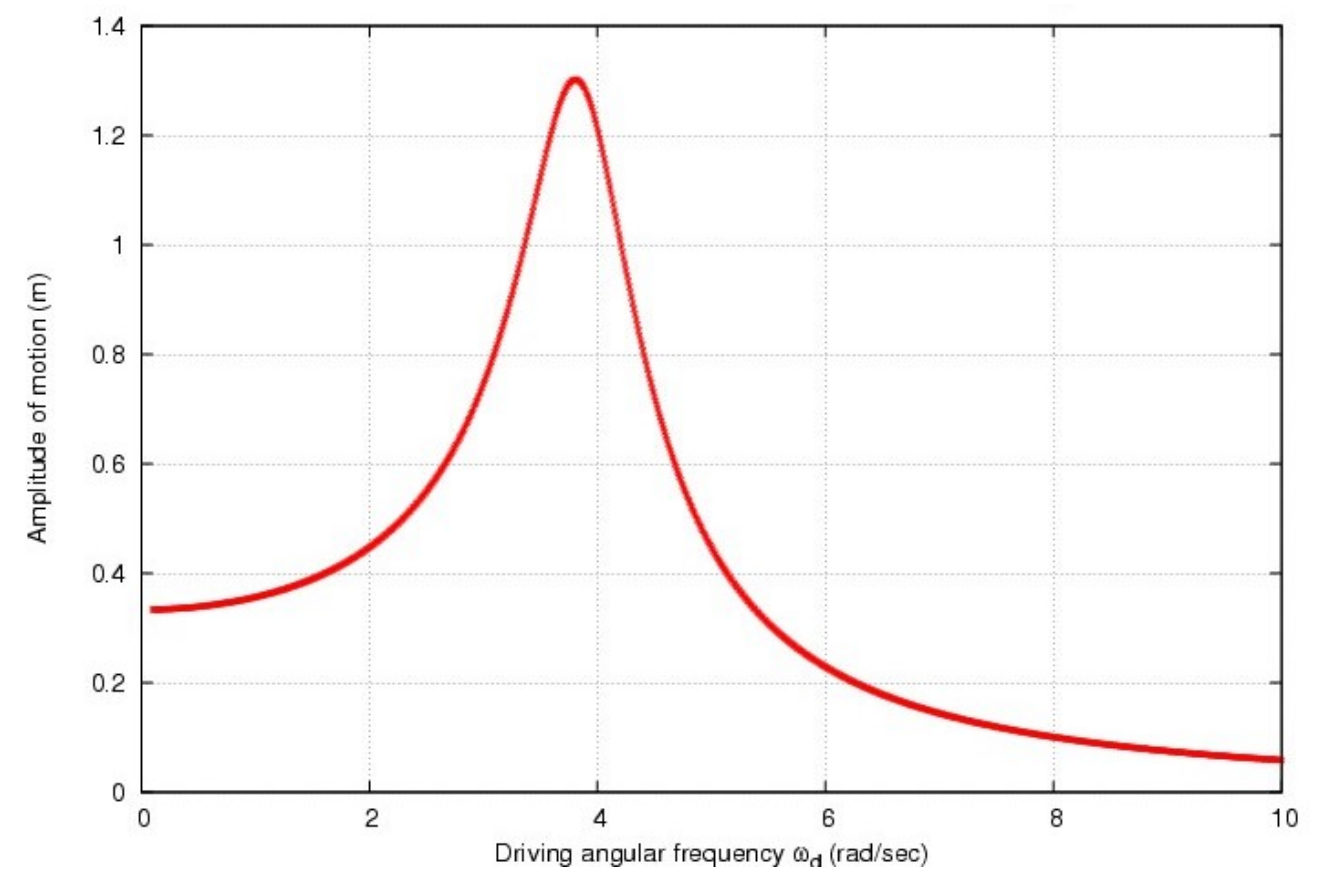

Figure 2.2: System with a resonance frequency of approximately 3.8 $\mathrm{rad} / \mathrm{sec}^{79}$ 
Resonance is of great importance when analyzing systems or structures that are subject to vibrations. At points of resonance, the amplification of the response causes large displacements and violent motion to the point where a system or structure can shake itself to pieces (see Figure 2.3). Whether a vibration source is mechanical (e.g. engine in a vehicle) or natural (e.g. tall building in an earthquake), the design of a system or structure should account for such resonant frequencies that may arise during their functional lifespan.

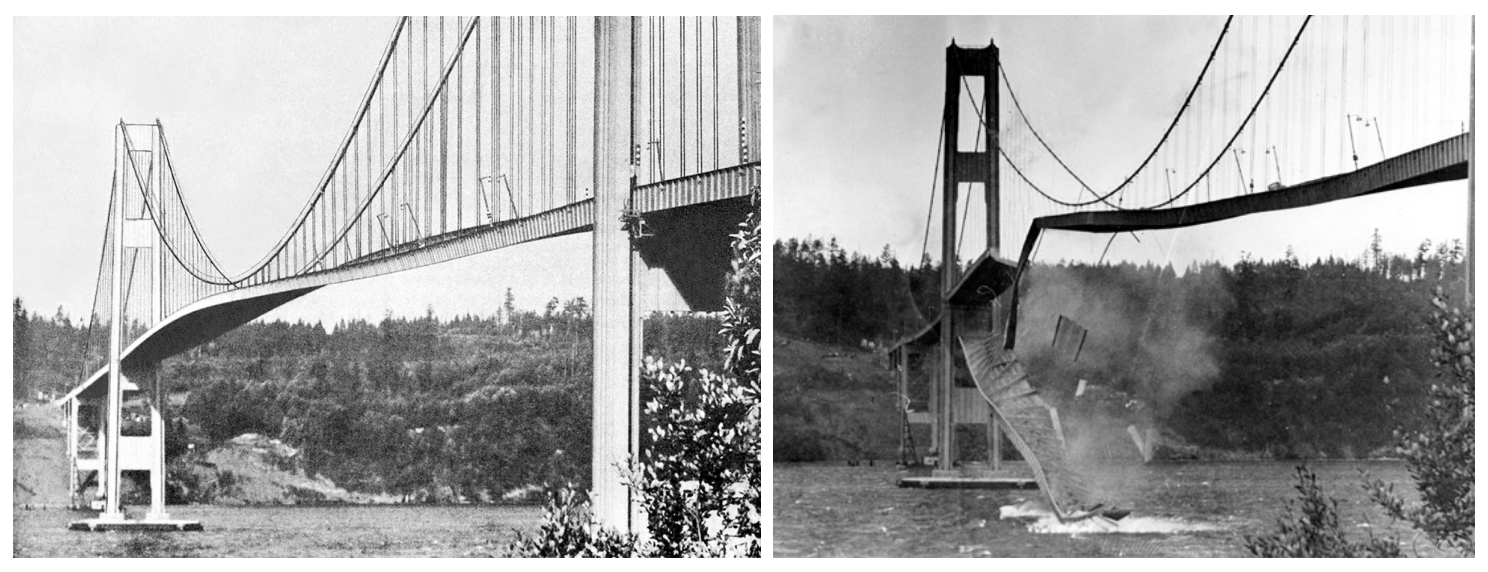

Figure 2.3: Tacoma Narrows Bridge in 1940 undergoing, in part, wind resonance $(\text { left })^{5}$ prior to structural collapse (right). ${ }^{36}$

Two special cases arise in an undamped spring-mass system under harmonic excitation, which are realized as follows. By setting the initials conditions $x_{0}$ and $v_{0}$ to zero in Equation 2.1, the general solution $x(t)$ becomes

$$
x(t)=\frac{f_{0}}{\omega_{n}^{2}-\omega^{2}}\left(\cos \omega t-\cos \omega_{n} t\right)
$$

Using a trigonometric identity, the solution $x(t)$ can then be re-written in form

$$
x(t)=\frac{2 f_{0}}{\omega_{n}^{2}-\omega^{2}} \sin \left(\frac{\omega_{n}-\omega}{2}\right) t \sin \left(\frac{\omega_{n}+\omega}{2}\right) t
$$


In the first case, note that as $\omega$ approaches $\omega_{n}$, the first sin term of Equation 2.3 oscillates with a much larger period $T$ than the second $\sin$ term (recall $T=2 \pi / \omega)$. As such, the response is a constructive and destructive interference of two sine waves, also known as beats. The largest amplitude of the beat period $T_{\text {beat }}$ occurs at

$$
\frac{2 f_{0}}{\omega_{n}^{2}-\omega^{2}}
$$

which is the maximum value of Equation 2.3. The beat phenomena (see Figure 2.4) has proven to be useful in many applications including string instruments, and the development of vehicle speed radar.

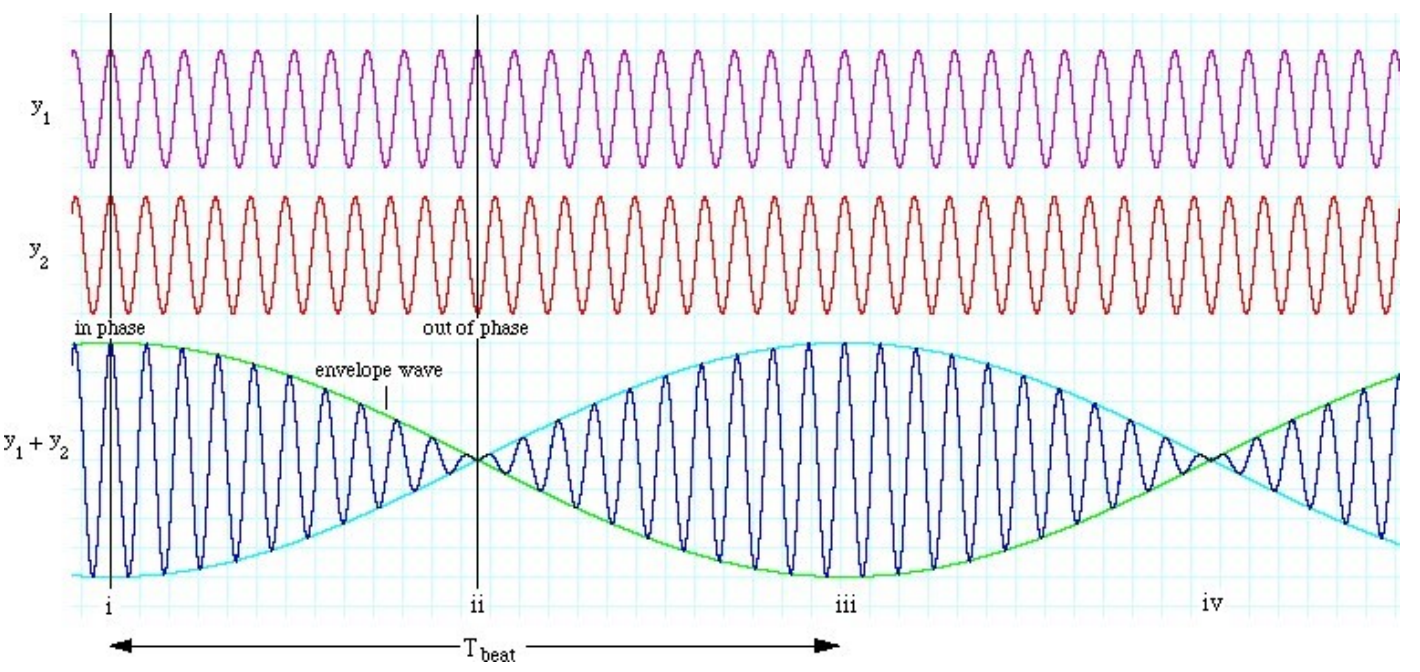

Figure 2.4: Beats are a constructive and destructive sum of two different frequencies. ${ }^{43}$

For the second (and more dangerous) case where the driving frequency $\omega$ is equal to the natural frequency $\omega_{n}$ of the system, terms in the original solution

$$
x(t)=\frac{v_{0}}{\omega_{n}} \sin \omega_{n} t+\left(x_{0}-\frac{f_{0}}{\omega_{n}^{2}-\omega^{2}}\right) \cos \omega_{n} t+\frac{f_{0}}{\omega_{n}^{2}-\omega^{2}} \cos \omega t
$$


become undefined which makes Equation 2.1 no longer valid. The solution must be derived again, where the particular solution $x_{p}$ takes the new form

$$
x_{p}(t)=t X \sin \omega t
$$

and is re-applied to the standard form of the equation of motion (EOM) for a springmass system with an acting force. After solving, the new solution to the system becomes

$$
x(t)=\frac{v_{0}}{\omega} \sin \omega t+x_{0} \cos \omega t+\frac{f_{0}}{2 \omega} t \sin \omega t
$$

where $\omega$ is equal to $\omega_{n}$. For this case, note that the amplitude

$$
\frac{f_{0}}{2 \omega} t
$$

has a slope term $t$ that grows without bound (see Figure 2.5), which at last defines the reason why resonance can be such a destructive force.

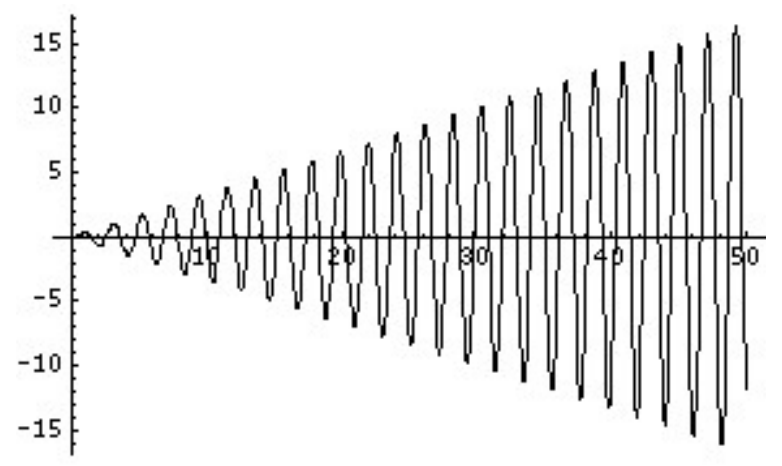

Figure 2.5: The amplitude of resonance continues to grow unbounded. ${ }^{46}$

CubeSats, especially their sensitive instruments, are susceptible to damage from such resonant frequencies. For example, circuit boards can warp and fatigue to the point of failure. Hardware can also become loose at these frequencies and rattle within the structure, possibly damaging nearby equipment. So, determining these resonant frequencies (and their effects) is of great benefit for the design of a CubeSat. 


\subsubsection{Free Response of Damped Systems}

From vibration theory, the damping ratio $\zeta$ is one of the most elusive characteristics of any vibration system. It helps determine the damping condition of the system, specifically, whether the system is underdamped $(\zeta<1)$, critically damped $(\zeta=1)$, or overdamped $(\zeta>1)$ (see Figure 2.6). This information is key for design optimization and mitigating adverse effects of vibration.

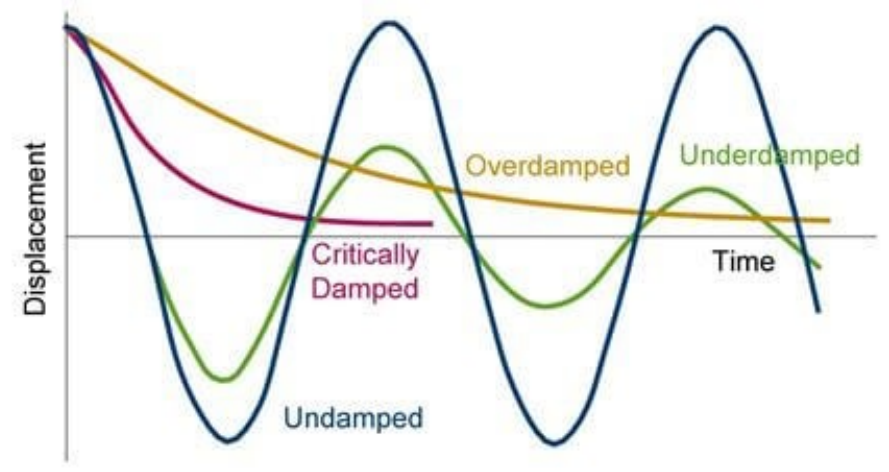

Figure 2.6: Response plots with various damping conditions. ${ }^{80}$

For most structures exposed to vibration, the underdamped response is preferred. An undamped system would never come to rest, a critically damped system is almost impossible to achieve in nature, and the abruptness of an overdamped system could damage sensitive equipment. CubeSats tend to fall in the underdamped category, and various damping factors can affect how the system reaches steady state (see Appendix A.2 for more details). Note that the underdamped response of Figure 2.6 has peak amplitudes that diminish over time. This rate of decay is a key characteristic of underdamped systems and is known as the exponential decay curve, where the decay "envelope" indicates the nature of the damping ratio $\zeta$ for the system (see Figure 2.7). A system with a $\zeta$ value close to 1 decays rapidly over time, while a system with a $\zeta$ value closer to 0 decays much slower over the same time frame. 


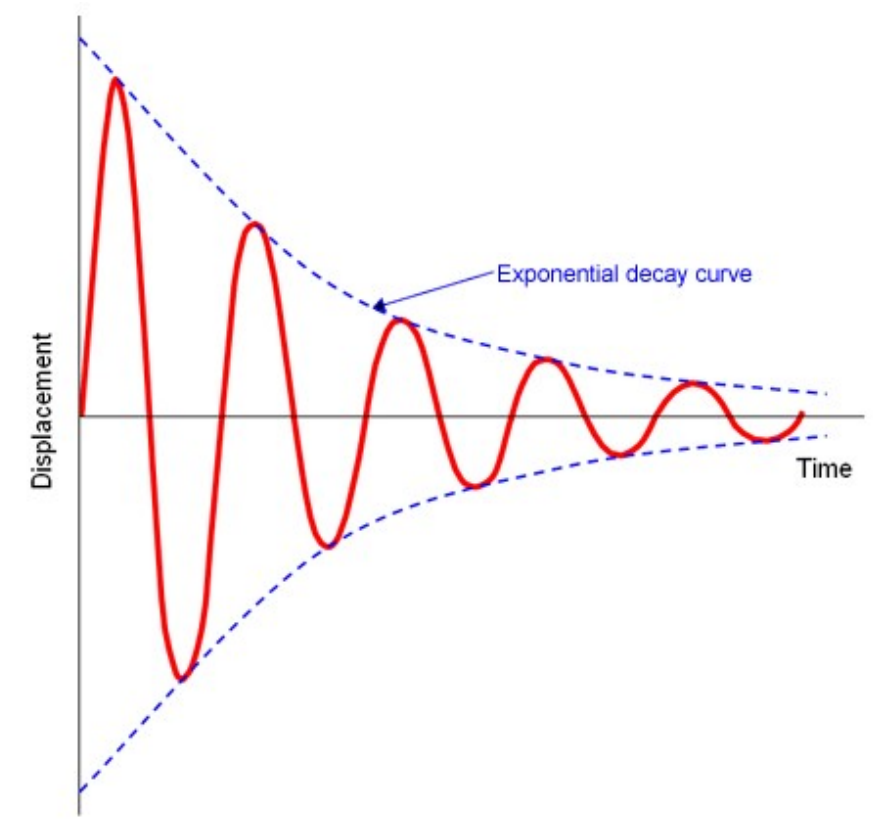

Figure 2.7: Exponential decay curve of an underdamped system. ${ }^{31}$

The damping ratio $\zeta$ can not be measured directly in a static state, or determined analytically. Instead, the analysis requires manipulation of dynamic experimental data to approximate its value for a given system. If underdamped response data is available, like that of Figure 2.7, then a curve-fit can be used in conjunction with the decay envelope $A e^{-\zeta \omega_{n} t}$ to solve for $\zeta$.

For dynamic response data over a frequency range (revert to Figure 2.1), different damping ratios can be present, depending on the number of degrees of freedom available in the system. In this case, each peak must analyzed individually in order to determine all the significant $\zeta$ values of the system. This can be achieved by using the half power bandwidth method, where

$$
\zeta=\frac{\omega_{2}-\omega_{1}}{2 \omega_{n}}
$$


Taking one peak amplitude $A$ and dividing by $\sqrt{2}, \omega_{1}$ and $\omega_{2}$ can be approximated from the data (see Figure 2.8) then substituted into Equation 2.6 to solve for $\zeta$. This process should be repeated for all significant peaks in the frequency response data.

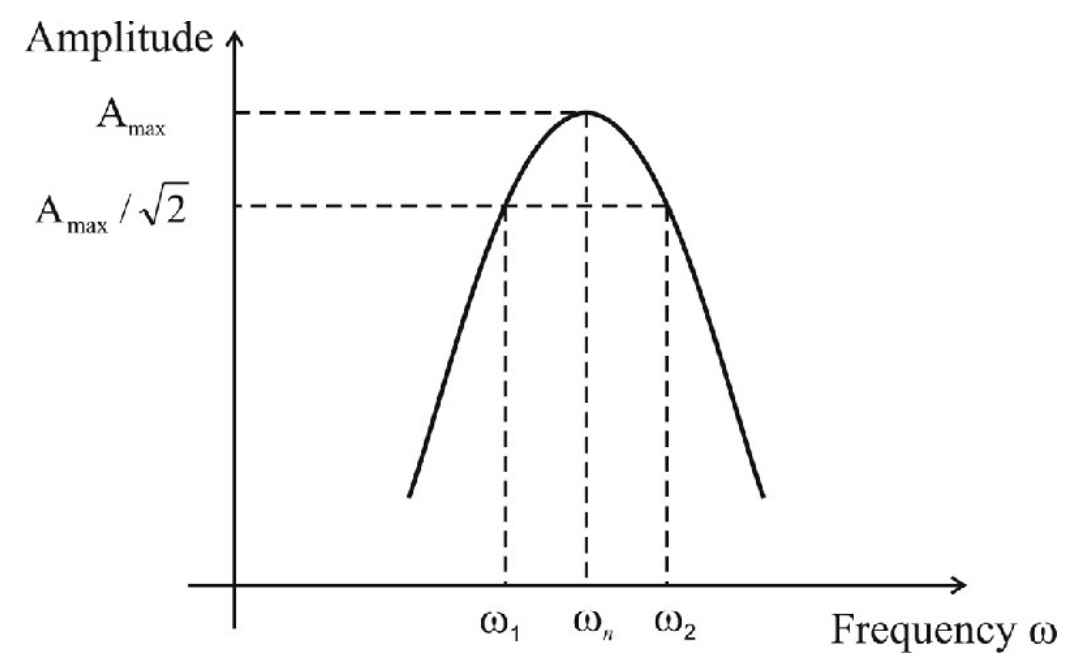

Figure 2.8: Half-power bandwidth method used to approximate the damping coefficient $\zeta$ of a vibration system. ${ }^{28}$

This analysis can be done for CubeSats using the dynamic response data collected directly from vibration tests, or simulated using computer models that can be built to represent the real system (discussed in Chapter 3). In either case, plotting response data and analyzing the damping characteristics of the response can give insight on the design of the structure and/or assembly for proof of concept and validation. Understanding the damping behavior of CubeSats can help assess how the levels of oscillation induced during a launch phase can affect critical components. Changes can then be made accordingly to ensure that their function will not be interrupted by such levels of oscillation. 


\subsubsection{Linear vs Nonlinear Analysis}

Nonlinear systems are difficult to analyze due to the added complexity of their behavior, in particular, because such systems have more than one possible equilibrium point of oscillation. In static systems, there are several factors that may contribute to the nonlinear behavior of a system, and a change in the "stiffness" of the system is a strong indicator that some of these factors may be present. Examples of such factors include friction, nonlinear geometry due to large deformations, the use of nonlinear materials, buckling, and contact stresses in nonlinear supports. If large deformations are observed (more than 1/20th of the largest dimension) ${ }^{76}$ then it can be assumed the the stiffness is changing, and as such, a nonlinear analysis is the best approach to avoid overdesign of a system. These same rules apply to the analysis of dynamic systems, but exceptions can be made in both cases. In a shake table test for example, if the deflections of a CubeSat are small about the equilibrium point, then it is safe to assume that the stiffness of the structure does not change significantly. So, a nonlinear analysis is thus not required, and permits the use of a linear dynamic analysis to approximate the behavior of a CubeSat. Here are some additional notes: ${ }^{84}$

- Nonlinear systems have more than one equilibrium point, each either stable or unstable.

- Steady-state does not always exist in nonlinear systems, solution strongly depends on the initial conditions $x_{0}$ and $v_{0}$.

- Period $T$ of nonlinear systems depends on $x_{0}, v_{0}$, and excitation amplitude $A$, unlike in linear systems which depend only on mass, damping, and stiffness.

- Resonance frequency of a nonlinear system may not be the same as the natural frequency of the linear system.

- Cannot use the superposition method in a nonlinear system.

- Harmonic excitation may cause chaotic motion in nonlinear systems. 


\subsubsection{Multiple-Degree-of-Freedom Systems}

While fundamental theory models can be derived using one or two degrees of freedom (DOF), it becomes apparent that such is not the case in nature. Many systems, whether natural or mechanical, may require an analysis using multiple degrees of freedom in order to better understand their behavior (see Figure 2.9). CubeSats fall under this category, as there are many factors that can influence their response, which may not always be the same in all respective axes.

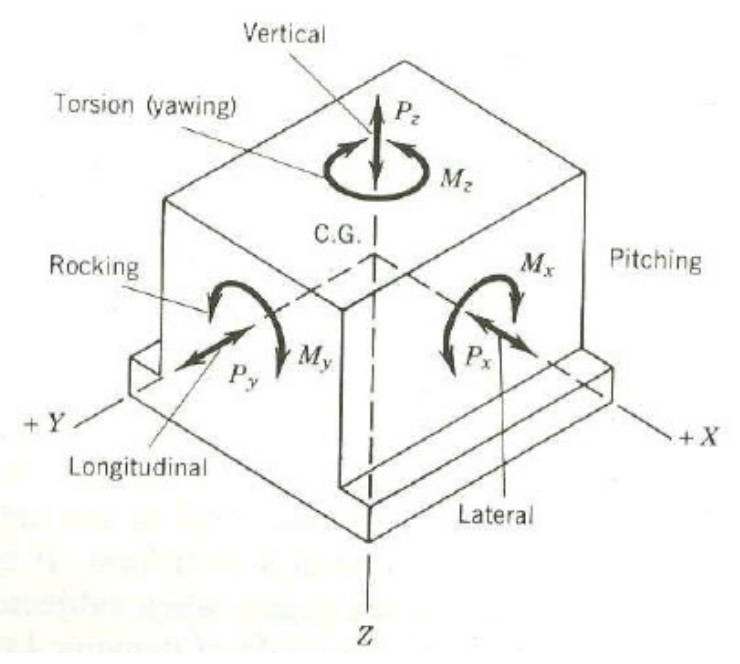

\section{Figure 2.9: Block mass with 6 Degrees of Freedom (DOF) ${ }^{36}$}

The development of the equations of motion for even 1-DOF systems can be quite cumbersome, and adding degrees of freedom to the analysis may require tremendous effort and time in order to reach a solution. For cases where many DOF's are needed for the analysis, it is convenient to instead use matrix notation to develop a system

solution. Matrix notation not only has the benefit of reducing the number of equations that need to be written out, but it can also be used in conjunction with computer software programs that can calculate a solution in a fraction of the time it would take to do by hand. 
Using the EOM for a spring-mass system in free vibration (see Appendix A.1 for more details),

$$
M \ddot{\mathbf{x}}(t)+K \mathbf{x}(t)=0
$$

note that $M$ and $K$ now represent the mass matrix and stiffness matrix, respectively, of the system where

$$
M=\operatorname{diag}\left(m_{1}, m_{2}, \ldots, m_{n}\right)
$$

and

$$
K=\left[\begin{array}{cccccc}
k_{1}+k_{2} & -k_{2} & 0 & 0 & \ldots & 0 \\
-k_{2} & k_{2}+k_{3} & -k_{3} & & \vdots \\
0 & -k_{3} & k_{3}+k_{4} & & \\
\vdots & & & k_{n-1}+k_{n} & -k_{n} \\
0 & & \ldots & -k_{n} & k_{n}
\end{array}\right]
$$

The EOM for a system with $n$ masses and $n$ spring elements can be written as

$$
m_{i} \ddot{x}_{i}+k_{i}\left(x_{i}-x_{i-1}\right)-k_{i+1}\left(x_{i+1}-x_{i}\right)=0, \quad i=1,2, \ldots, n
$$

The solution $x(t)$ takes on the vector form

$$
\mathbf{x}(t)=\left[\begin{array}{c}
x_{1}(t) \\
x_{2}(t) \\
\vdots \\
x_{n}(t)
\end{array}\right]
$$

which is representative of the individual solutions for the $n$-DOF system. This set of solutions can be quite large for complex assemblies like CubeSats, and carrying out such calculations can prove to be challenging. However, this convenient matrix approach of solving multi-DOF systems can be adopted into the powerful finite element method. 


\subsection{Finite Element Approach}

Analyzing the stresses and displacements of structural components under loading can require a great deal of computations, especially with systems that have multiple degrees of freedom. In such cases, matrix notation helps organize the mathematical expressions that arise from the analysis. However, these expressions alone cannot describe how loads are distributed throughout a structural system. For this, it is necessary to introduce the finite element method (FEM), which breaks a component into smaller finite elements and analyzes each individual element to predict the behavior of the component as a whole, a procedure also known as discretization. ${ }^{85}$

There are several element shapes that can be used in FEM (see Figure 2.10), and the best choice depends on the application and type of analysis that is being performed. FEM is not limited to the analysis of stresses and displacements, as it can also be applied to structural dynamics, thermal systems, fluid mechanics, electrical systems, or a combination of these areas. This is one of the many appeals of FEM, and when combined with computer software that can present pictorial results, the application of FEM can be quite powerful in the field of engineering.
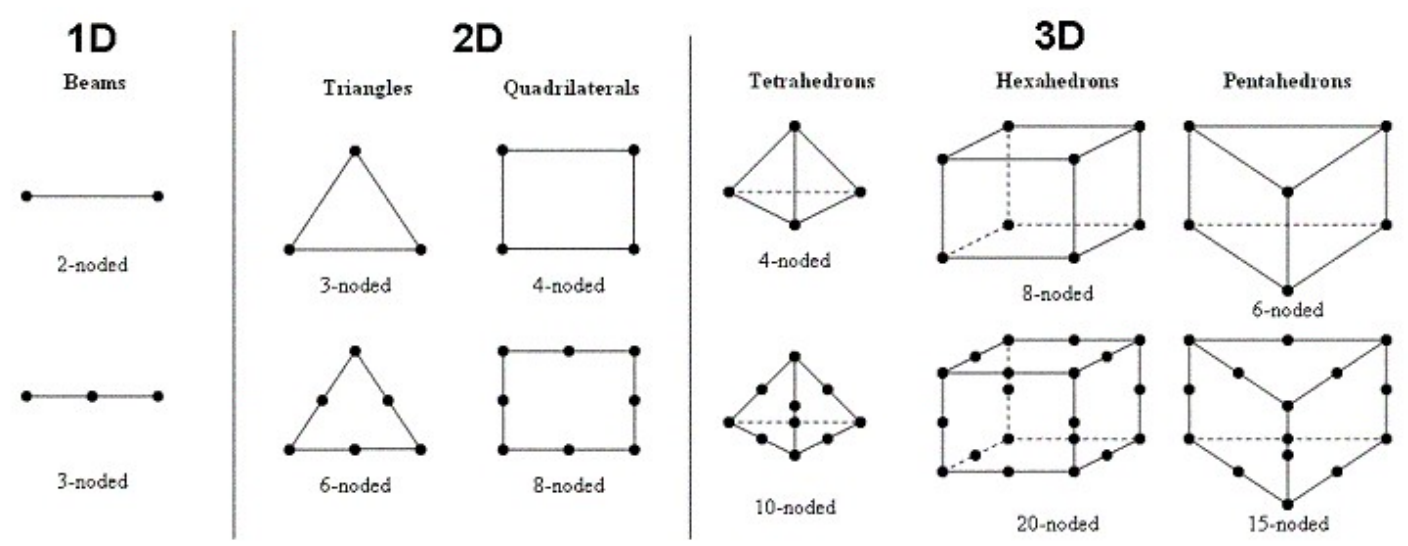

Figure 2.10: Simple element shapes used to discretize a body into finite elements. ${ }^{67}$ 


\subsubsection{Structural Dynamics}

Bringing the discussion full circle, FEM will now be applied to the analysis of structural dynamics. As mentioned in the previous section, FEM has many applications that go beyond stress analysis, and the importance of FEM continues to grow in industry and research to this day. This section focuses on the use of FEM in dynamic analysis (see Figure 2.11), and will tie in with the vibration theory discussed earlier in this chapter.

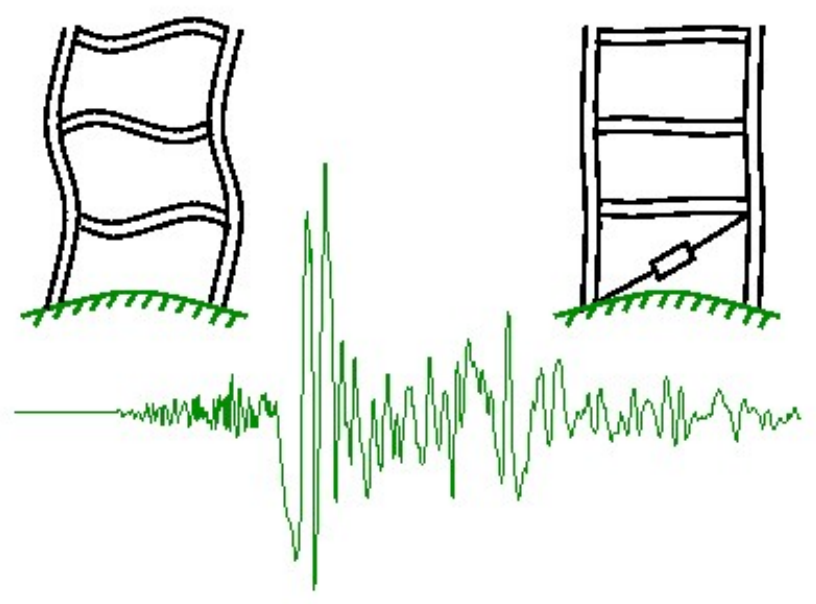

Figure 2.11: Example of a structure undergoing dynamic excitation. ${ }^{68}$

From vibration theory, the standard equation of motion (EOM) for a second order spring-mass system can be written as

$$
m \ddot{x}+k x=F(t)
$$

where $m$ is the mass, $\ddot{x}$ is the second derivative of displacement (i.e. acceleration), $k$ is the spring constant, and $F$ is the force being applied to the system (see Appendix A.5 for more details). Dividing all terms in Equation 2.12 by $m$, note

$$
\omega^{2}=\frac{k}{m}
$$


where $\omega$ is the natural frequency of the spring-mass system. Furthermore,

$$
\tau=\frac{2 \pi}{\omega}
$$

where $\tau$ (or $T$ ) is the period of oscillation for the system.

For the dynamic finite element analysis, a simple bar element (see Appendix B.1.2 for more details) will be used to set up an example. The EOM for the spring-mass system is first re-written in local coordinates as

$$
\left\{f^{e}(t)\right\}=[k]\{d\}+[m]\{\ddot{d}\}
$$

where

$$
[k]=\frac{A E}{L}\left[\begin{array}{rr}
1 & -1 \\
-1 & 1
\end{array}\right]
$$

is the stiffness matrix for a bar element and

$$
[m]=\frac{\rho A L}{2}\left[\begin{array}{ll}
1 & 0 \\
0 & 1
\end{array}\right]
$$

is the lumped mass matrix. By applying the energy method to the bar element, it can also be shown that

$$
[m]=\frac{\rho A L}{6}\left[\begin{array}{ll}
2 & 1 \\
1 & 2
\end{array}\right]
$$

where Equation 2.18 is the consistent mass matrix for a bar element. Note that the lumped mass matrix of Equation 2.17 consists of diagonal terms only, and though it requires less computations, it sacrifices the accuracy that is otherwise available using the consistent mass matrix.

Assembling the bar elements in global coordinates, the EOM becomes

$$
\{F(t)\}=[K]\{d\}+[M]\{\ddot{d}\}
$$

where $\{F\},[K]$, and $[M]$ are the sums of $\left\{f^{e}\right\},\left[k^{e}\right]$, and $\left[m^{e}\right]$, respectively. 
With the global equation of the EOM fully assembled and taking the determinant

$$
\left|[K]-\omega^{2} M\right|=0
$$

it is possible to solve for the natural frequency $\omega$ of a dynamic system. Note that Equation 2.20 represents a set of $n$ algebraic equations, where $n$ is the number of DOF for the model. As an example, take a cantilever bar composed of two 1-D bar elements (see Figure 2.12)
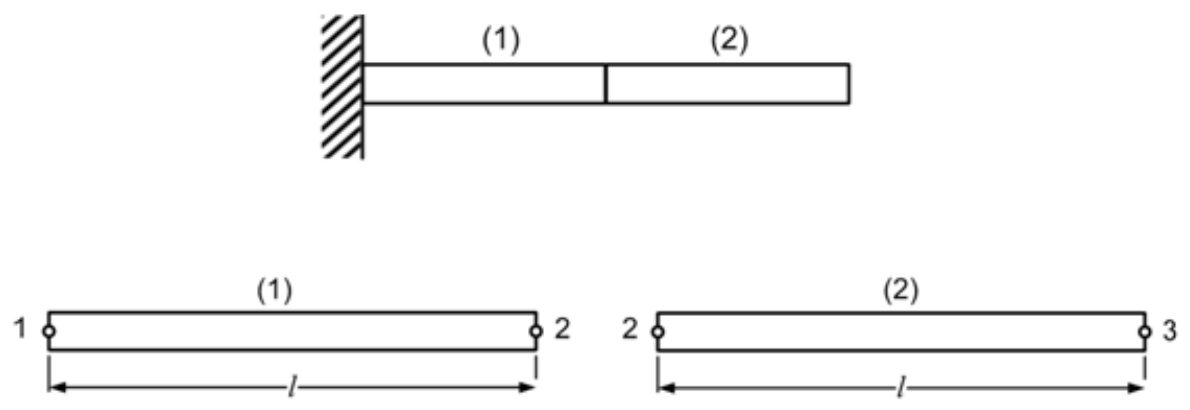

Figure 2.12: Cantilever bar composed of two elements. ${ }^{50}$

with cross-sectional area $A$, mass density $\rho$, elastic modulus $E$, and length $L$. Applying the lumped mass matrix (Equation 2.17) for simplicity, it can be shown that the determinant becomes

$$
\left|\frac{A E}{L}\left[\begin{array}{rr}
2 & -1 \\
-1 & 1
\end{array}\right]-\lambda \frac{\rho A L}{2}\left[\begin{array}{ll}
2 & 0 \\
0 & 1
\end{array}\right]\right|=0
$$

Letting $\lambda=\omega^{2}$ and $\mu=E /\left(\rho L^{2}\right)$, the determinant reduces to

$$
\left|\begin{array}{cc}
2 \mu-\lambda & -\mu \\
-\mu & \mu-\frac{\lambda}{2}
\end{array}\right|=0
$$

Solving the determinant,

$$
\lambda=2 \mu \pm \mu \sqrt{2}
$$

where

$$
\lambda_{1}=0.60 \mu \quad \text { and } \quad \lambda_{2}=3.41 \mu
$$


Here, $\lambda_{1}$ and $\lambda_{1}$ are the eigenvalues of the solution, and can be used to solve for the natural frequencies of the system (recall $\lambda=\omega^{2}$ ). Comparing these values to the classic solution $\lambda=0.616 \mu,{ }^{85}$ it is evident that an FEM model can approximate real systems to a significantly accurate degree. While systems with $n$-DOFs will have $n$ natural frequencies $\omega$, note that each $\omega$ of a dynamic system will correspond to a particular geometry shape, known as a mode shape of the system. The study of these modes and their corresponding natural frequencies is referred to as modal analysis, which is used to investigate the dynamic properties of a structures or system (see Figure 2.13). As a general rule, at least twice the number of elements as the number of frequencies desired should be used for the dynamic analysis. ${ }^{85}$

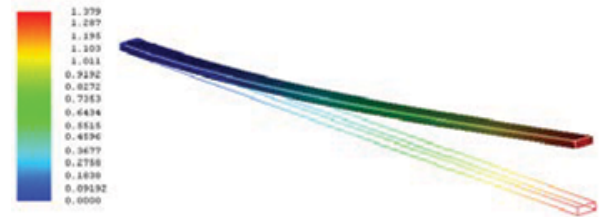

a) $1^{\text {st }}$ mode shape

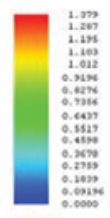

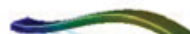

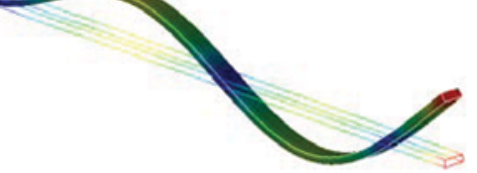

c) $3^{\text {rd }}$ mode shape

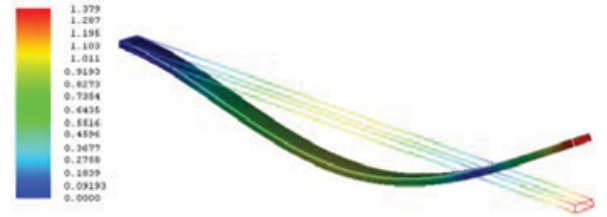

b) $2^{\text {nd }}$ mode shape

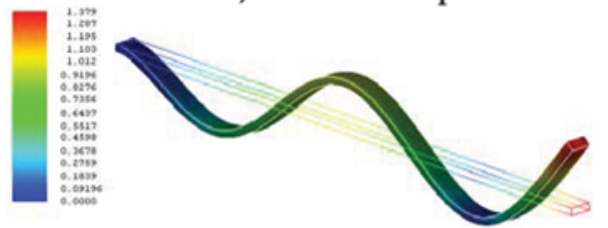

d) $4^{\text {th }}$ mode shape

Figure 2.13: Modal analysis depicting the first four mode shapes of a cantilever beam. ${ }^{49}$

While the analysis in this section was limited to a simple cantilever bar, the same equations can be developed for more complex structures like CubeSats, where 3D solid elements can be used with FEM software to model the dynamic behavior of the structure (see Appendix B.4 for more details). The lower modes and natural frequencies of a CubeSat are generally of interest, as the accuracy of higher frequencies tends to suffer from noise. However, cautious engineering judgment should be practiced to avoid overseeing frequencies that may be destructive to the system. 
Chapter 3

\section{DESIGN METHODOLOGY}

\subsection{Concept}

In order to establish a basis of validation for the CubeSat FEM models to be created, a series of experiments were first performed in a lab setting, where the collected data could be used to evaluate the accuracy of the FEM models. The test pod and mass model are pseudo structures that can be used to represent the P-Pod and CubeSat, respectively, in a vibrations test. As such, these pseudo structures were used to perform all dynamic tests, and their FEM models were created accordingly.

Again, the main objective of the FEM model here is not only to evaluate the dynamic performance of a CubeSat early in the design phase (see Figure 3.1), but to also analyze how the response is affected by the surrounding components in the assembly. To see the potential benefits of using this as a design tool, the CubeSat CPX structure was created and applied to the FEM template established from the experimental models. In addition, CubeSat CPX also provides conceptual design features that may facilitate manufacturing and assembly of future CubeSat structures.

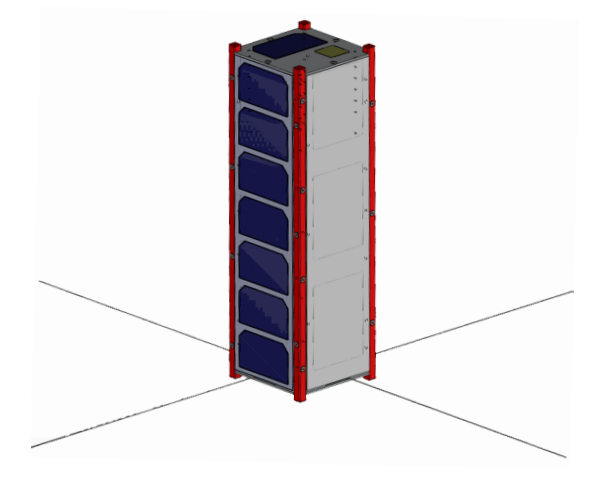

Figure 3.1: Rendering of a 3U CubeSat design. ${ }^{2}$ 


\subsection{Modeling the Test Setup}

Geometry models were created to represent the test pod, mass model, and fixture plate of the vibration test setup. In order to simplify the analysis of the FEM models, computer aided design (CAD) models were made for the three components. The mass and main dimensions were collected and applied to the CAD models, along with the material properties of each corresponding part. All models were built using SolidWorks 3D, which is a software used to create CAD geometries and assemblies. The CAD models for the vibration table were generously provided by Unholtz-Dickie Corp. (see Figure 3.2), and although they were used during the early stages of the FEM simulation, they were not applied to the final FEM models. Nonetheless, they were essential in understanding how vibration table systems work, and how their design and application are critical for vibration testing.

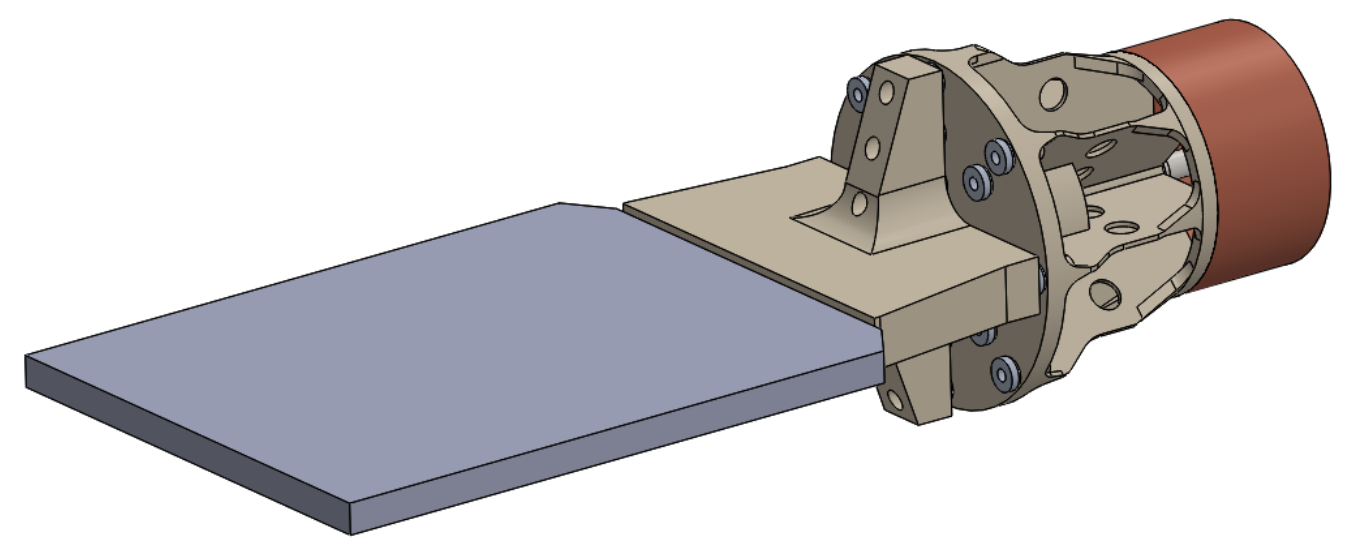

Figure 3.2: SolidWorks CAD model of the vibration table armature assembly. ${ }^{77}$ 


\subsubsection{Test Pod}

For preliminary vibration testing of CubeSats, the Cal Poly CubeSat lab uses a simpler and more robust test pod as a substitute for the more complex and expensive P-Pod (see Figure 3.3). However, final dynamic tests are always performed using the P-Pod once CubeSats qualify for P-Pod integration upon clearing additional tests. For modeling purposes, all experiments here were performed using the test pod configuration.
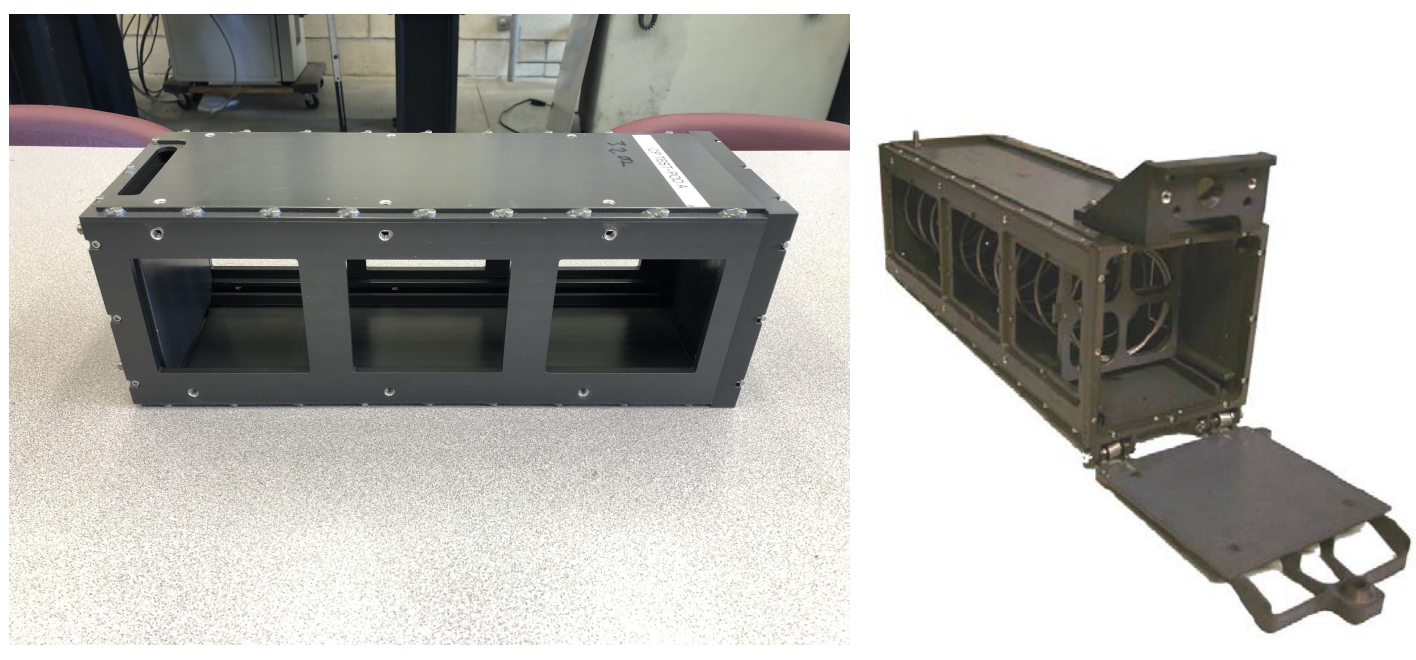

Figure 3.3: $3 U$ Test Pod (left) and P-Pod (right) ${ }^{22}$ used to house CubeSats for dynamic testing.

The test pod is a $130 \mathrm{~mm} \times 130 \mathrm{~mm} \times 366 \mathrm{~mm}$ structure with a mass of $3.2 \mathrm{~kg}$. The structure is composed of Aluminum 6061-T6 and is anodized using a hard Teflonimpregnated coating which helps prevent cold welding between the test pod and loaded CubeSat. Two bolt patterns are available on the structure, including a 6bolt pattern used in previous missions, and the now preferred 8-bolt pattern. All experiments here used the 6-bolt pattern, as this was the pattern that matched the fixture available at the time of testing. 
Similar to the "jack-in-the-box" spring-base system of the P-Pod, the test pod uses a "floating" plate and a set of guide rails to restrain a CubeSat during testing (see Figure 3.4). Although the floating plate is restricted to one side of the test pod, the clearance within its range of motion ensures that the plate will come in full contact and sit flush with a CubeSat. This is assuming that the dimensions of the CubeSat fall within the allowed geometric tolerances.

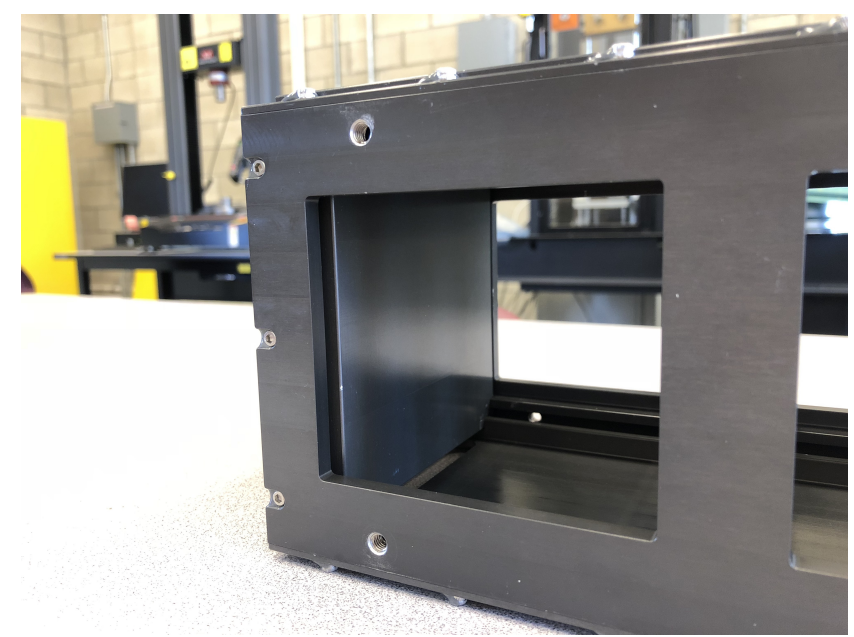

Figure 3.4: Floating plate and guide rails used to constrain CubeSats.

Four spring plungers are used to control the clamping force that is applied by the floating plate onto the CubeSat (see Figure 3.5). Spring plungers are torqued to specification using a torque wrench, where each spring plunger of the test pod can apply a force of up to 58 Newtons (13 lbf) ${ }^{66}$ while fully compressed. Staking, which is a form of epoxy, is generally applied to all bolts including spring plungers to prevent them from shaking loose during dynamic testing. However, other types of thread locking (like Loctite) may be used, but should be done with caution as these type of thread lockers tend to produce more debris during testing ${ }^{22}$ which may compromise the delicate systems of the CubeSat. Visual "witness marks" can also be used to inspect for loose hardware, where lines are drawn on hardware before testing and inspected for mismatch after testing is completed. 

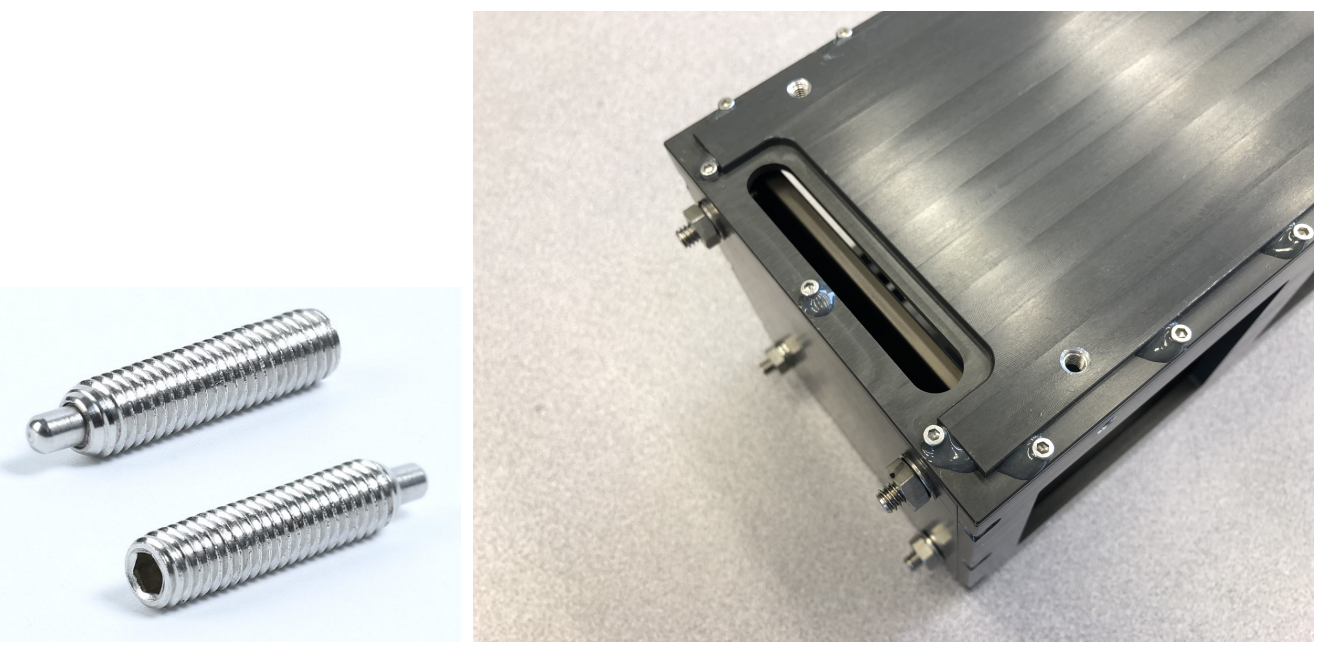

Figure 3.5: Spring plungers (left) ${ }^{36}$ press onto the floating plate (right).

Unlike the hinged lid of a P-Pod, a test pod has a simple cap that is held in place with a series of screws (see Figure 3.6). With the CubeSat loaded, the cap is replaced and the screws are torqued to specification using a torque wrench. To prevent damage to the delicate CubeSat panels that interface with the floating plate and cap of the test pod, the design standard requires that CubeSats have a set of stand off protrusions at both ends of the CubeSat structure. As such, the floating plate and cap apply the clamping force to the stand offs and avoid crushing the panel surfaces.

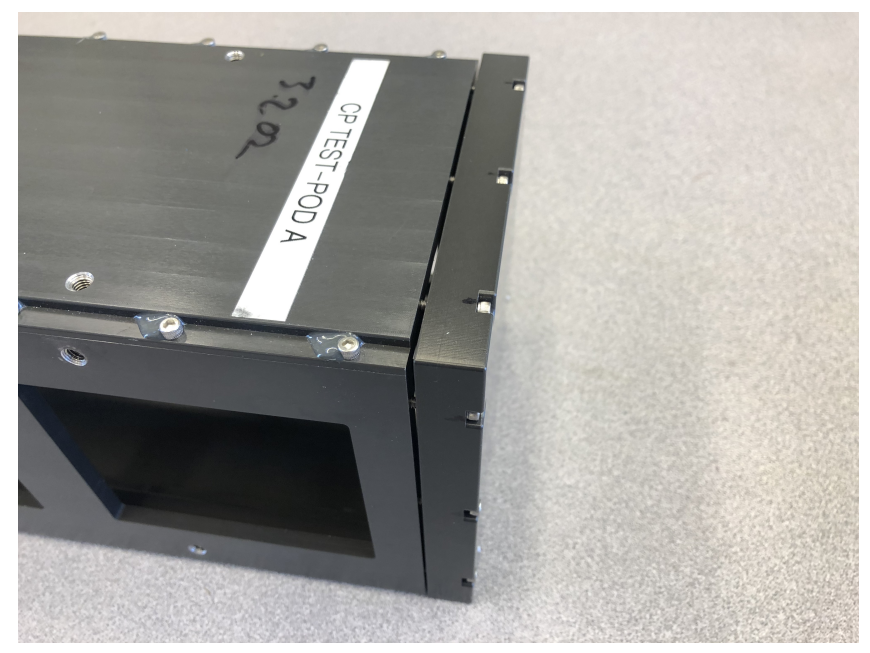

Figure 3.6: Removable cap of test pod. 
There are no "bumpers" on the inside of the test pod (or P-Pod) structure to help cushion the vibration of a CubeSat. The reason for this is that damping materials like rubber have the adverse effect of outgassing, where trapped gas within the material is suddenly released when exposed to a vacuum environment, like space. The released gas can condense on lens and/or damage the electrical systems of a CubeSat, which may jeopardize the mission objectives of the satellite. NASA has established a list of materials and parameters to help minimize the effects of outgassing, and all space systems (like CubeSats) are designed around these principles.

Although a $3 \mathrm{U}$ test pod was used for all experiments here, it can also accommodate individual 1U CubeSats, just like a P-Pod structure. If more space is needed, but not enough to warrant an extra $1 \mathrm{U}$ compartment, a "tuna can" adapter can be bolted to the test pod for extra space. ${ }^{22}$ Besides the $3 \mathrm{U}$ test pod, other test pod sizes are available for testing purposes.

The CAD model for the test pod was built from scratch, using measured dimensions of the test pod to give the CAD model a general form (see Figure 3.7). Wall thicknesses were recorded, including the body, cap, and spring plunger wall. The guide rails of the test pod were also measured and replicated in the CAD model, as these are key features that set part of the boundary conditions for a CubeSat when loaded.

While CAD dimensions were kept as close as possible to those of the real test pod, the main driver of the design was the mass of the CAD model. Like the test pod, aluminum material properties were applied to the model to reflect the mass and density of the test pod. Also, the thickness of the spring plunger wall was increased in the model to compensate for the mass of the floating plate in the test pod, which sits near the same wall. With the model completed, its mass was evaluated as $3.2 \mathrm{~kg}$ (7.06 lbs) in SolidWorks, which matches the measured $3.2 \mathrm{~kg}$ mass of the test pod. 

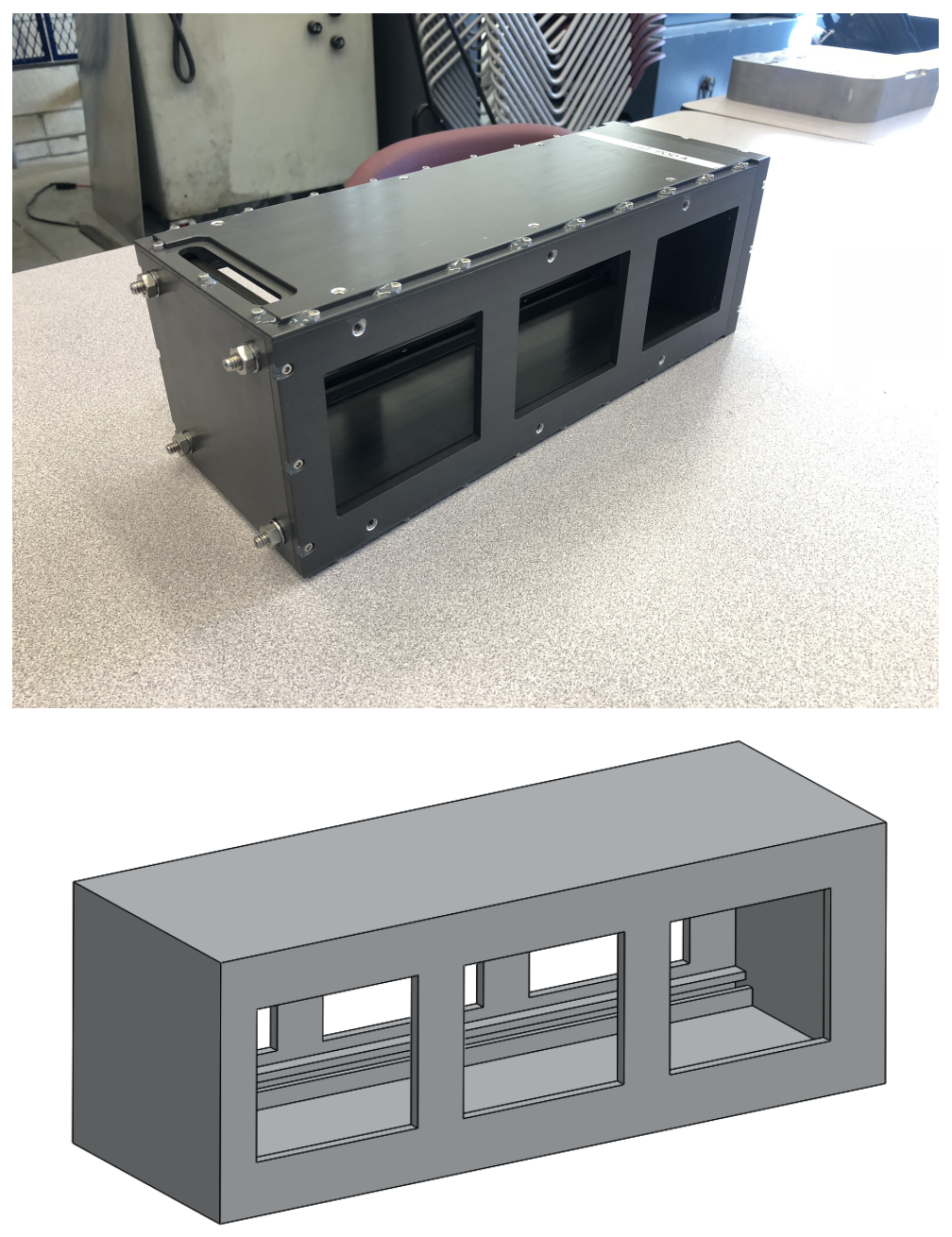

Figure 3.7: Test pod (top) and the corresponding CAD model (bottom). 


\subsubsection{Mass Model}

Sometimes it is necessary to analyze the dynamic behavior of a P-Pod or test pod, where a functional CubeSat is not necessarily required in order to extract the information desired from the system response. For such cases, a mass model can be used as a substitute for a CubeSat while still achieving accurate test results (see Figure $3.8)$.

The $3 \mathrm{U}$ mass model used for these experiments is a $100 \mathrm{~mm}$ x $100 \mathrm{~mm}$ x $340 \mathrm{~mm}$ solid structure composed of Delrin, which is a thermoplastic favored in engineering for its machinability, high stiffness, and low friction. Steel brackets are bolted to the body for added mass, bringing the total mass to $4.5 \mathrm{~kg}$ (9.9 lbs).
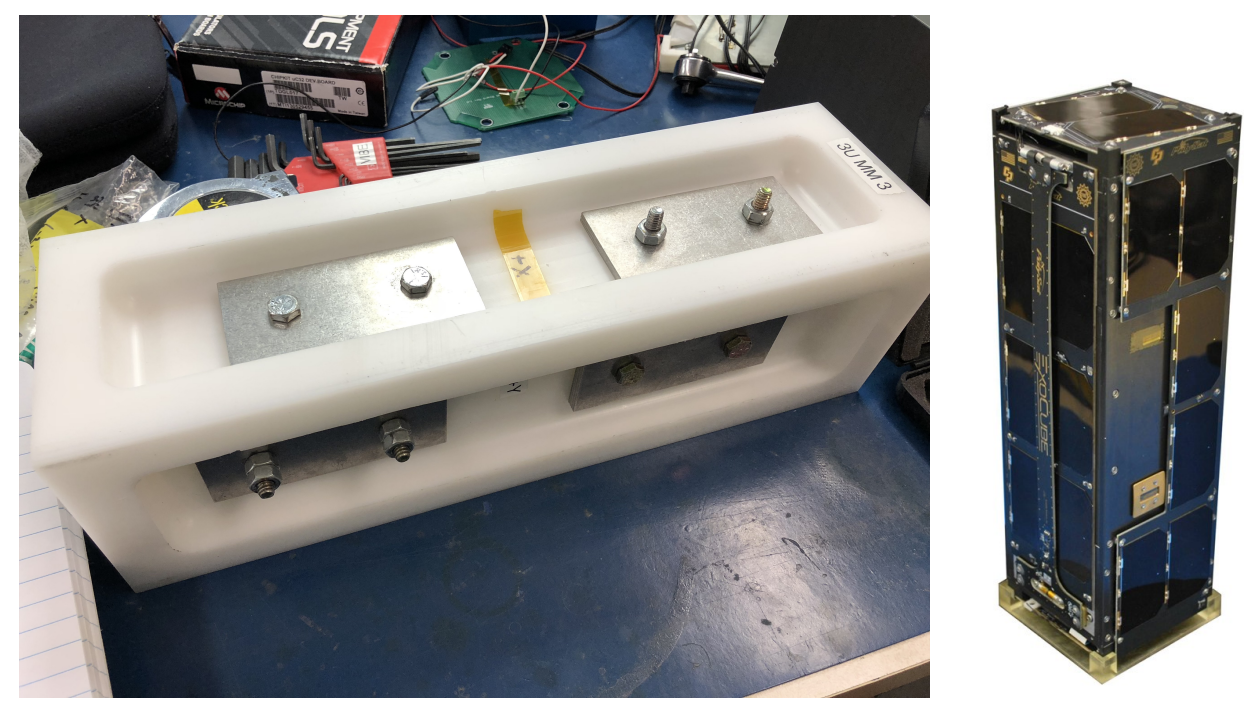

Figure 3.8: A 3U mass model (left) can be used as a substitute for CubeSats (right) ${ }^{23}$ in dynamic testing.

Although the mass model is a simplified version of a CubeSat, the dimensions still meet the CubeSat standard in order to avoid damaging the test pod or P-Pod during assembly and testing. Note that the mass model was also designed without stand off protrusions, as there are no fragile components to protect at these ends. 
To avoid the complexity of an assembly model with steel hardware, the CAD model for the mass model was instead designed as one solid body (see Figure 3.9). Applying the measured dimensions of the mass model, these same dimensions were used to create the body of the CAD model. Delrin material properties were applied to the model, and with the mass evaluated by SolidWorks as $4.5 \mathrm{~kg}$ (9.91 lbs), this matched the measured $4.5 \mathrm{~kg}$ of the mass model. The design of the CAD model was also made symmetric to reflect that of the mass model.

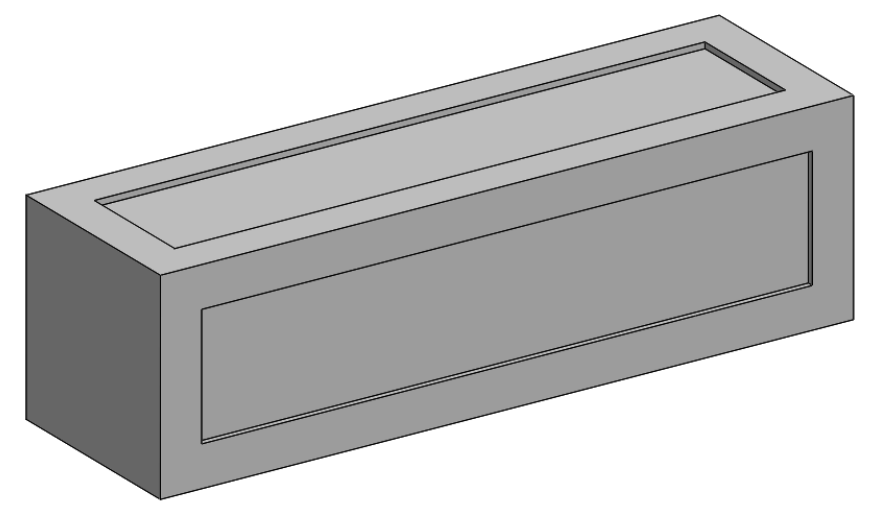

Figure 3.9: CAD geometry of the mass model. 


\subsubsection{Fixture Plate}

The fixture plate is the interface tool used to mount P-Pods and test pods to a shake table for vibration testing (see Figure 3.10). Made out of a solid block of magnesium AZ31B alloy and with a mass of $7.5 \mathrm{~kg}(16.5 \mathrm{lbs})$, the fixture provides an excellent stiffness-to-weight ratio, which is ideal for dynamic testing. The light weight of the material minimizes the effects of noise on the system response, and the high stiffness minimizes deformation that can adversely affect the accuracy of the response.

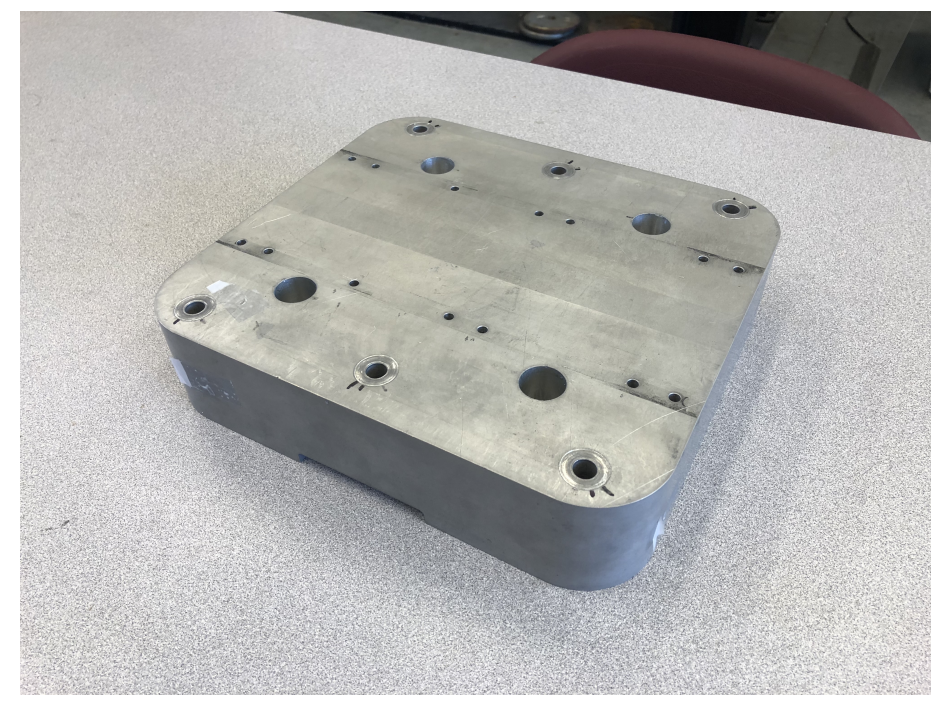

Figure 3.10: Fixture used to attach the test pod to the vibration table.

Like the test pod, the fixture plate has a 6 and 8-bolt pattern available, with the former pattern being used for all experiments performed in this work. The $63.5 \mathrm{~mm}$ x $254 \mathrm{~mm}$ x $279.4 \mathrm{~mm}$ fixture can be mounted to the slip plate of a horizontal shake table setup, or it can be bolted directly to the armature of a vertical shake table setup. This orientation depends on the type of dynamic test being performed, and the axes that need to be tested. Like the test pod, witness marks can be used on the fasteners that bolt down the fixture, and inspection of these markings is recommended before and after each test to check for loose hardware. The two "notches" of the fixture 
serve as grips (see Figure 3.11), and must face down when the fixture is bolted to the shake table.

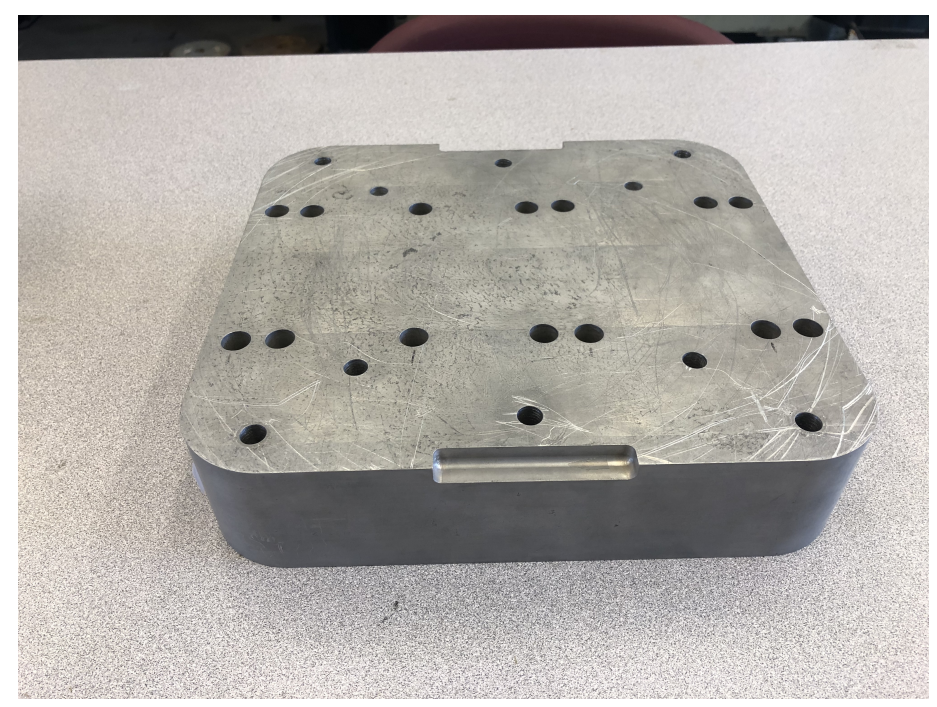

Figure 3.11: Bolt patterns on the bottom side of the fixture plate.

Note that although magnesium offers the benefits of both low weight and high stiffness when compared to other common metals like steel and aluminum, the manufacturing process of magnesium can be dangerous. Machining magnesium for example forms chips and dust that can ignite when exposed to a heat source, which may lead to a fire. ${ }^{33}$ Safe practices and precautions should be used when machining magnesium parts, and the work should only be done by a trained technician.

The fixture plate CAD model was a fairly straightforward design, applying the measured dimensions to create the main body (see Figure 3.12). Magnesium properties were assigned to the CAD model, and all thru-holes, bores, and grip notches were omitted to simplify the CAD design. Despite the omitted features, the mass was evaluated as $7.53 \mathrm{~kg}(16.59 \mathrm{lbs})$ in SolidWorks, which is still relatively close to the measured mass of $7.51 \mathrm{~kg}$ for the fixture plate. 

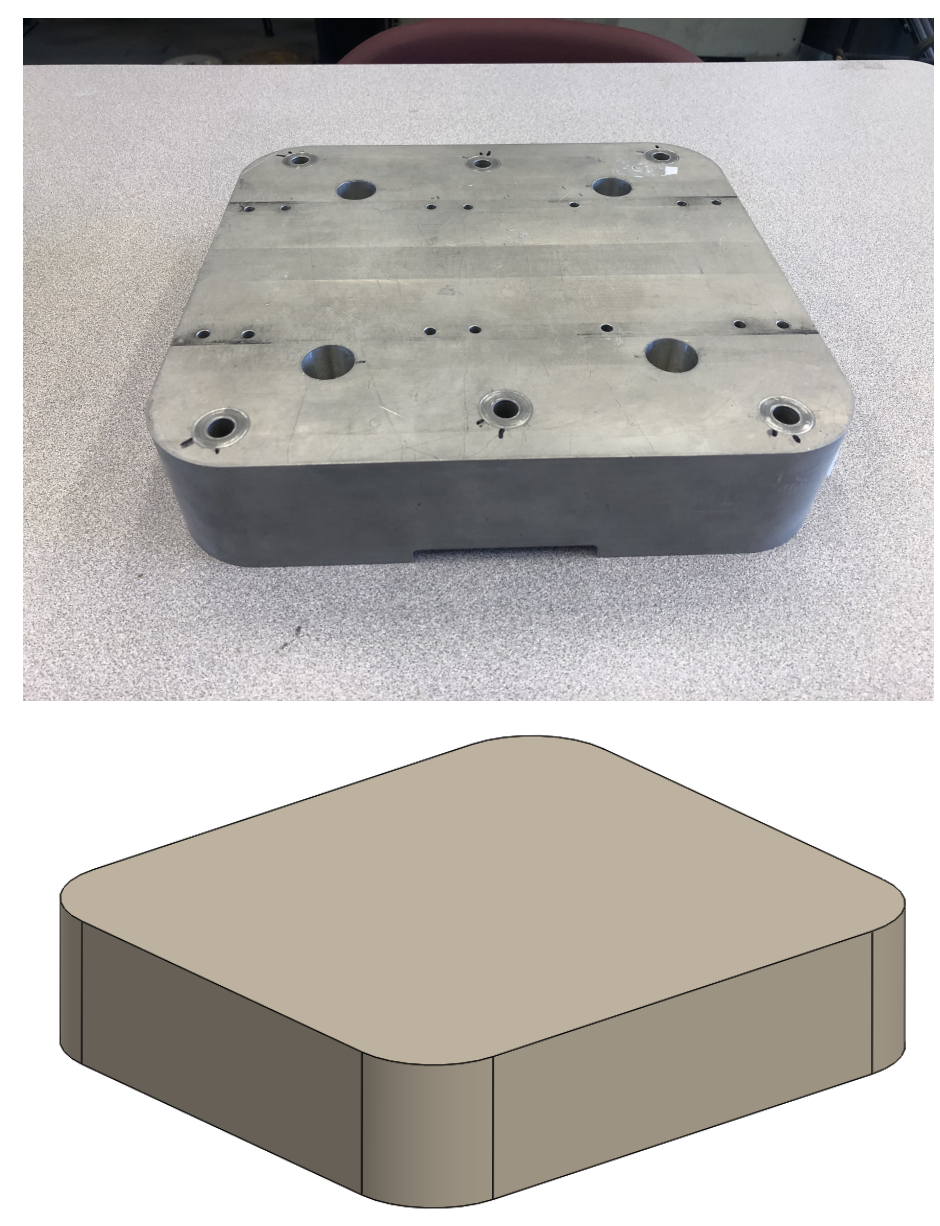

Figure 3.12: Fixture plate (top) and the corresponding CAD model (bottom). 


\subsubsection{Assembly}

With the CAD models completed for the test pod, mass model, and fixture plate, the corresponding assemblies were created to replicate the setups to be used during the vibration test runs. Position constraints were used to align all the components in an assembly, as they would in the real setup.

For the "loaded" test pod CAD assembly, the z-axis (along length) of the mass model was aligned with that of the test pod, and the adjacent walls between the test pod and mass model were set to be parallel with respect to one another (see Figure 3.13).
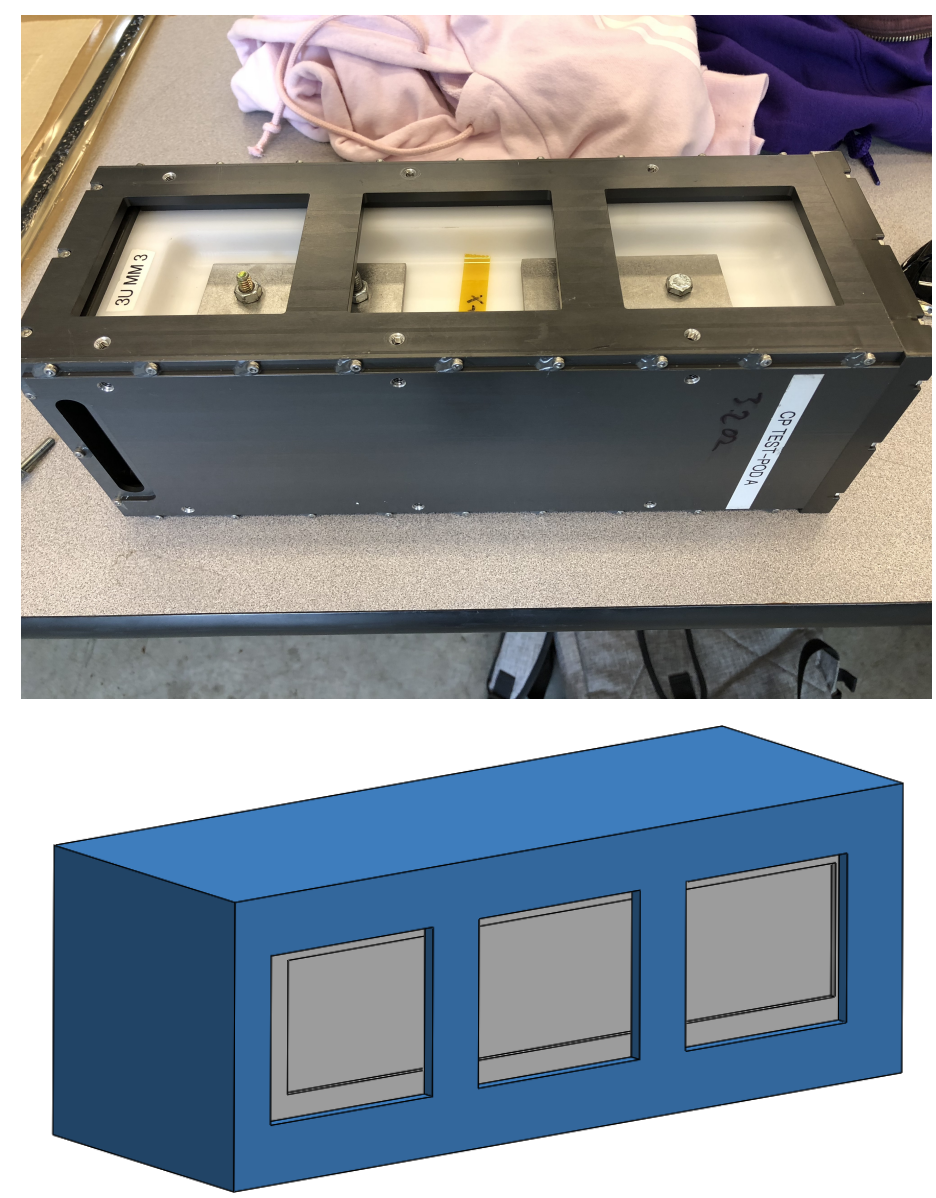

Figure 3.13: Loaded test pod (top) and the corresponding CAD assembly (bottom). 
To properly set the z-position of the mass model within the test pod, a constraint was created between the inner wall of the test pod cap and the face of the mass model (see Figure 3.14). These faces were set to be coincident, which now defined the location of the mass model in the z-axis.

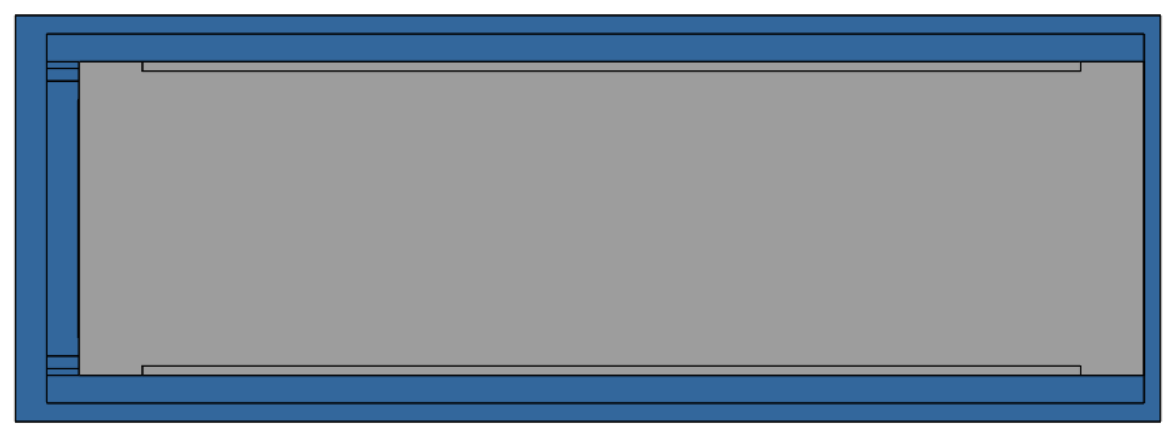

Figure 3.14: Cross-section of the CAD assembly showing the mass model is coincident with the inner cap wall (right side) of the test pod.

To constrain the position of the mass model in the $\mathrm{x}-\mathrm{y}$ plane, the walls of the mass model were set to be coincident with the faces of the test pod guide rails (see Figure 3.15). This prevents the mass model from rotating about its z-axis, and sets the location of the mass model in the x-y plane. With all axes restricted, the mass model was now fully constrained in the test pod CAD assembly.

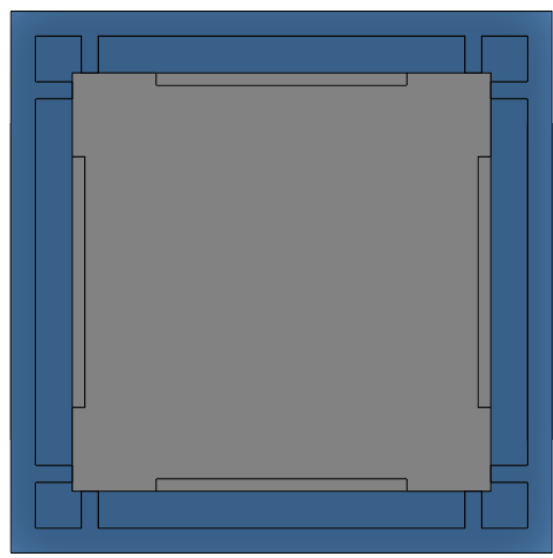

Figure 3.15: Cross-section of the CAD assembly showing the mass model is coincident with the guide rails of the test pod. 
Additional constraints were applied to the CAD assembly, where the bottom panel of the test pod was set to be coincident and centered with the top face of the fixture plate (see Figure 3.16). Note that the position of the test pod along the z-axis is slightly offset to the right of the fixture plate. This is due to the layout of the bolt pattern between the test pod and fixture plate, which cannot be adjusted. The offset was measured on the real parts, and applied to the CAD assembly as a distance constraint. With this final positioning, the test pod was now fully constrained to the fixture plate in the CAD assembly. The loaded test pod CAD assembly was built in the exact same way, but now to include the mass model along with the aforementioned constraints (see Figure 3.17).
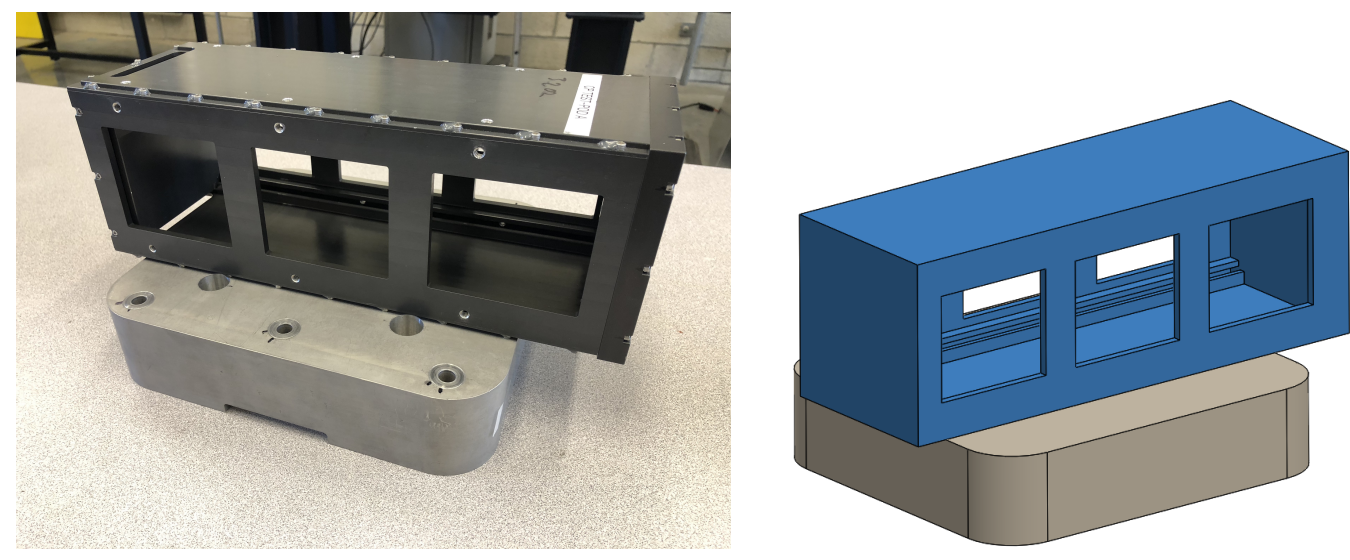

Figure 3.16: CAD assembly of the empty test pod with the fixture plate.
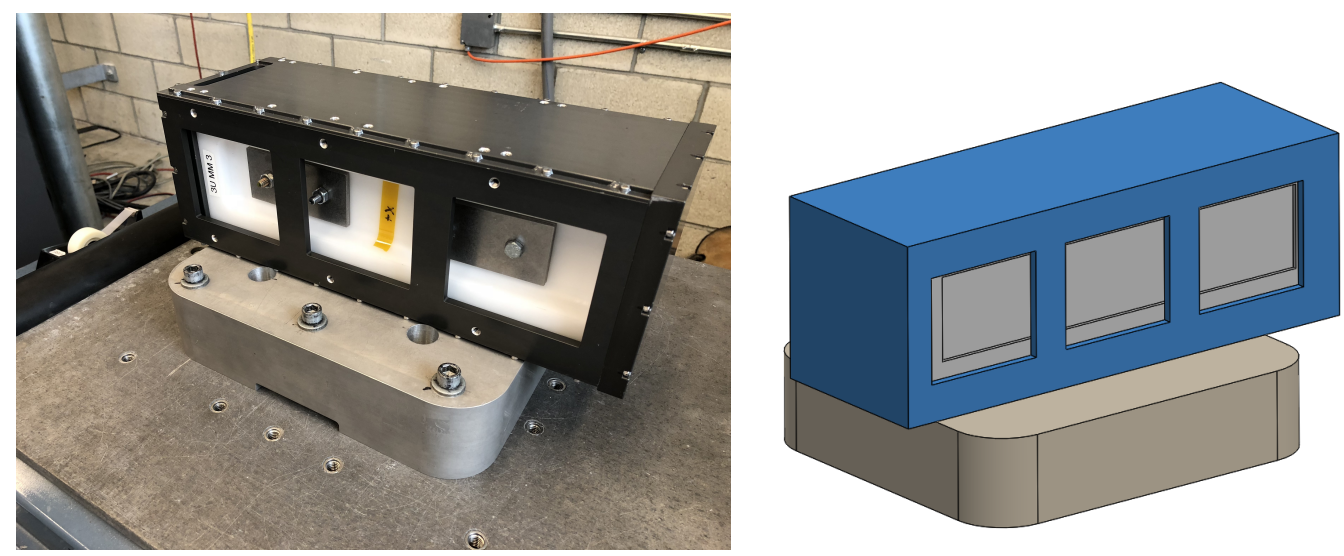

Figure 3.17: CAD assembly of the loaded test pod with the fixture plate. 


\subsection{Idea Behind a Concept Structure}

A concept structure was created to test the application of the FEA model template to be created, while also providing ideas that may help solve design challenges that have been observed in the past. It was adopted from an early revision of a Cal Poly SLO PolySat structure (see Figure 3.18), and focus was placed on re-designing components in order to help minimize manufacturing lead times, and facilitate assembly of the structure.
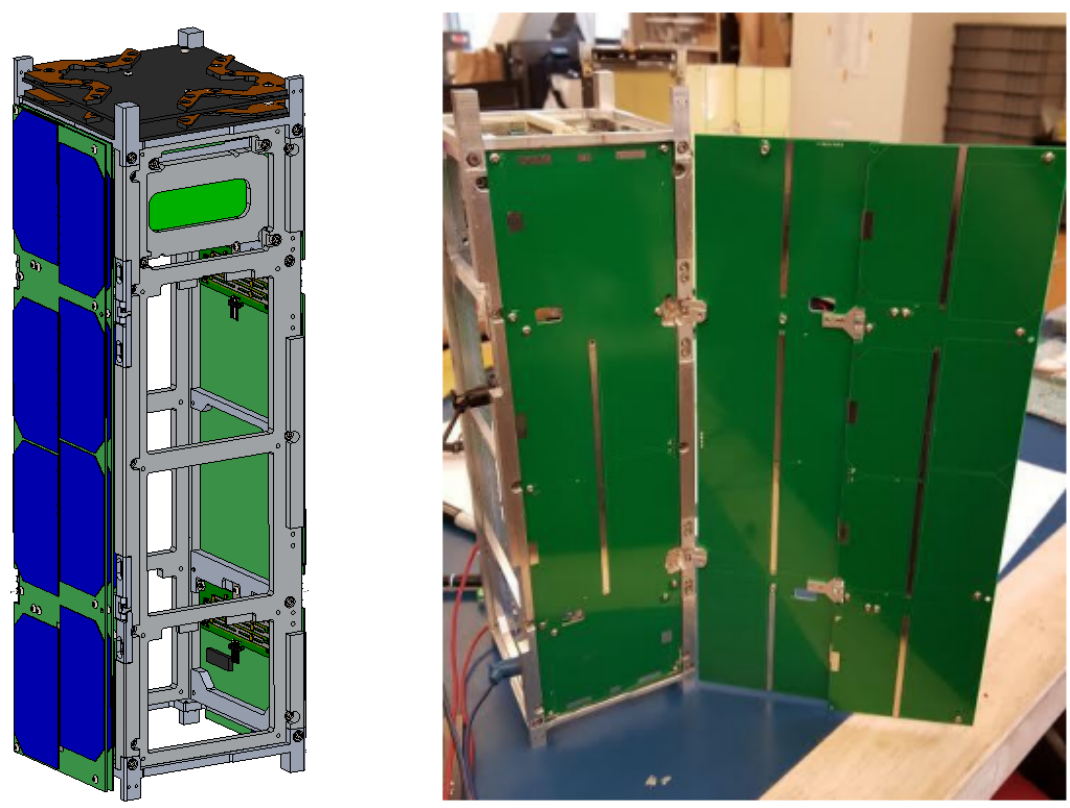

Figure 3.18: CAD assembly (left) of a Cal Poly SLO PolySat structure with deployable solar panels ${ }^{70}$ (right).

The design of a CubeSat can be quite intricate, depending on the payload and the capabilities required to sustain the given payload. This can complicate the structural design of a CubeSat, which can be costly, especially when revisions need to be made late in manufacturing and assembly. Removing all the non-metallic components of the CubeSat structure of Figure 3.18, note that the structure is assembled using many different components that are bolted together (see Figure 3.19). 

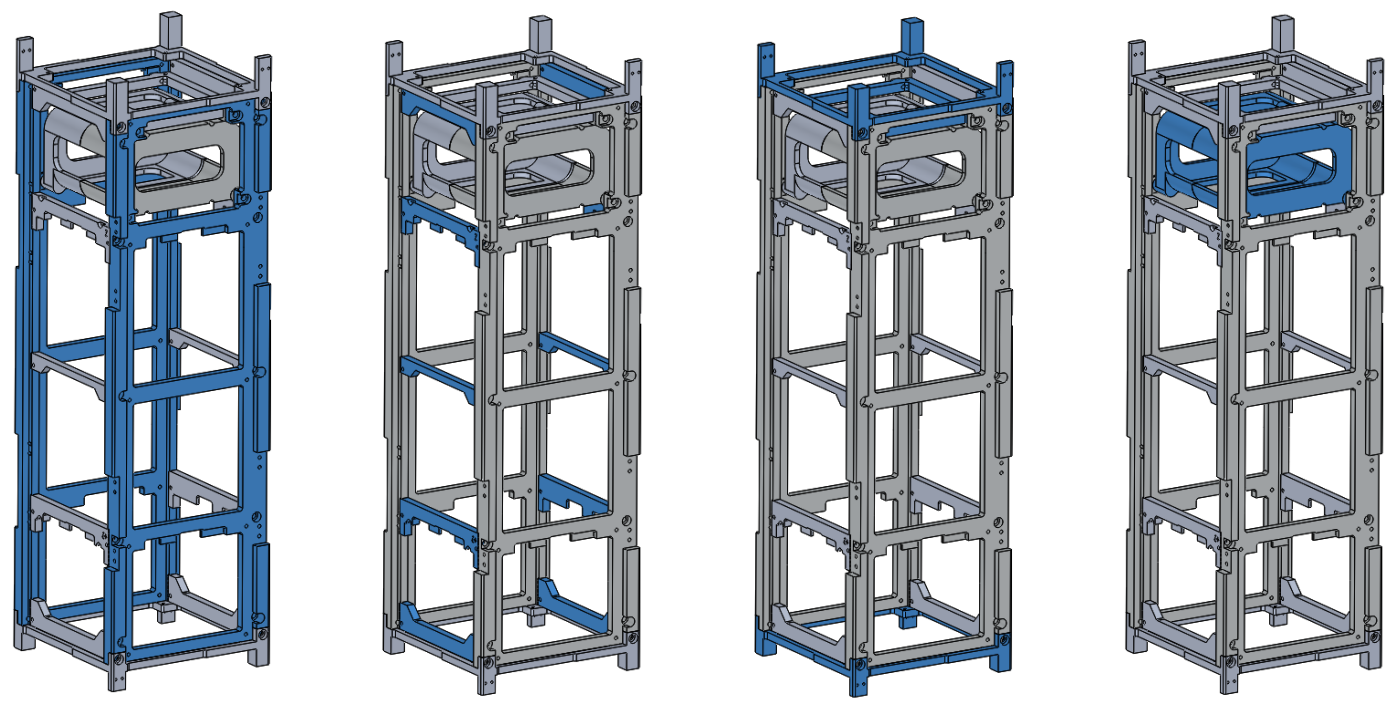

Figure 3.19: Main components of the PolySat structure.

The top of this particular structure is generally reserved for circuit boards, which serve as the "brain" of the CubeSat (revert to Figure 3.18). A battery compartment sits right below the circuit boards, which helps provide power to the subsystems of the CubeSat. The remaining open space is allocated for payloads, and maximizing this space makes a structure more versatile for payload integration.

From first-hand experience, the components of a CubeSat structure can be difficult to make, and require significant machining time. Assembly of a CubeSat structure with many components can also be cumbersome, and removing internal components (e.g. payloads) during fit checks and troubleshooting requires disassembly of most of the structure, which can be a time-consuming process. Taking these factors into consideration, the CubeSat CPX concept structure was created to help work towards a solution of these on-going CubeSat design challenges. 


\subsubsection{CubeSat CPX}

The driving factors of the CubeSat CPX design are modularity and machinability of the structure (see Figure 3.20). One of the first things to note is that the design reduces the number of components needed for the structure, while maximizing the space allowed for payloads. Minimizing the number of components is meant to facilitate assembly and simplify tolerance stacking, which is critical for a proper fit of all components. With the exception of the top and bottom stand-off components adopted from the PolySat structure of Figure 3.19, the components were re-designed and simplified for the CPX concept. Components were designed using mostly planar features, as contours and odd geometries can add significant machining time.

The mass of a CubeSat is critical for flight qualification, and payloads must be accounted for in the CubeSat design. The structure alone can add significant weight, and not designing around the intended payload can push to the total weight of the CubeSat beyond the limit established by the CubeSat standard. So ideal CubeSats structures are designed to be light, yet strong enough to support heavy payloads.
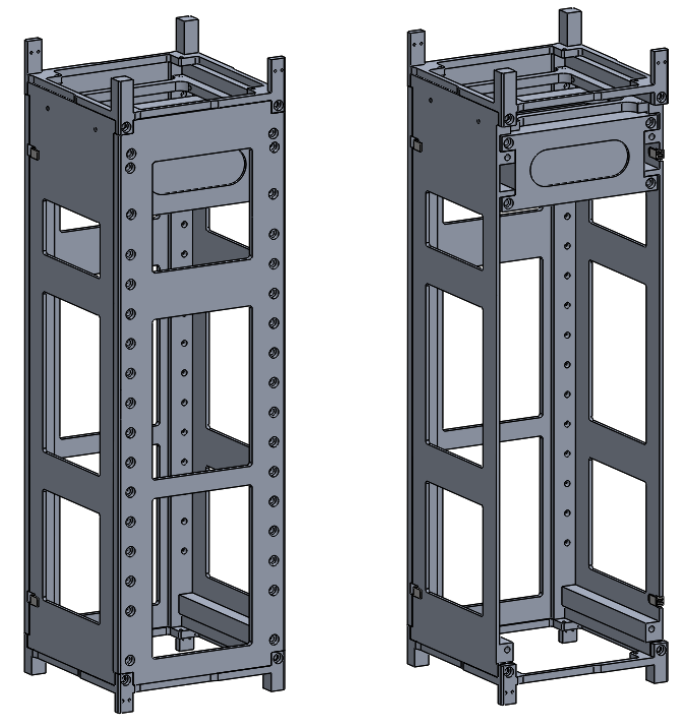

Figure 3.20: CAD assembly of the CubeSat CPX structure. 
The mounting panels of CPX are designed to be the main anchor points of payloads. While payloads are generally constrained to all four sides of a CubeSat structure, the thick mounting panels of CPX can provide sufficient stiffness to support a payload on their own (see Figure 3.21). More than one bolt pattern could also be implemented into the mounting panel design, as final assembly may require an adjustment to the position of the payload in order to make room for cables and other hardware not accounted for in a CAD assembly.

The panels are designed to be flat and symmetric for machining. With proper fixturing, the single panel allows for better control of tolerances, and reduces the number of fixtures that are otherwise needed to make different parts with various geometries. This single panel option can be cost-effective and time saving for manufacturing and assembly of the overall structure.
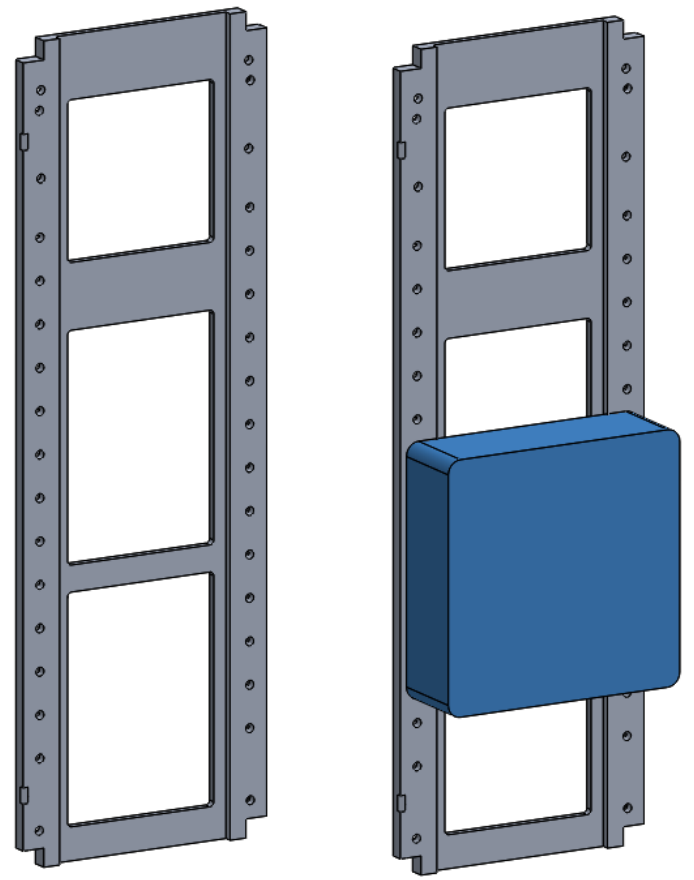

Figure 3.21: Mounting panel allows various installation positions for a payload. 
CPX can also accommodate various payload sizes and orientations, adding versatility to its application in CubeSat missions. Payloads are not restricted to the internal square perimeter of the structure, where rectangular payloads can be bolted directly to the mounting panels, and heavier payloads can still be attached to all four side panels of the CPX structure for added support (see Figure 3.22).

With internal space already limited by the metal structure and payload of a CubeSat, integration of the electronic sub-systems can prove to be challenging during assembly. Routing of cables/wires can be especially difficult, as components like the battery bracket and payload can restrict the line of sight to the two extreme panels of the CubeSat. As depicted in Figure 3.22, using the mounting panels as the main anchor points would allow room for cables to be routed along the center of the structure and even between payloads, with less strain on the wire leads and connection points.

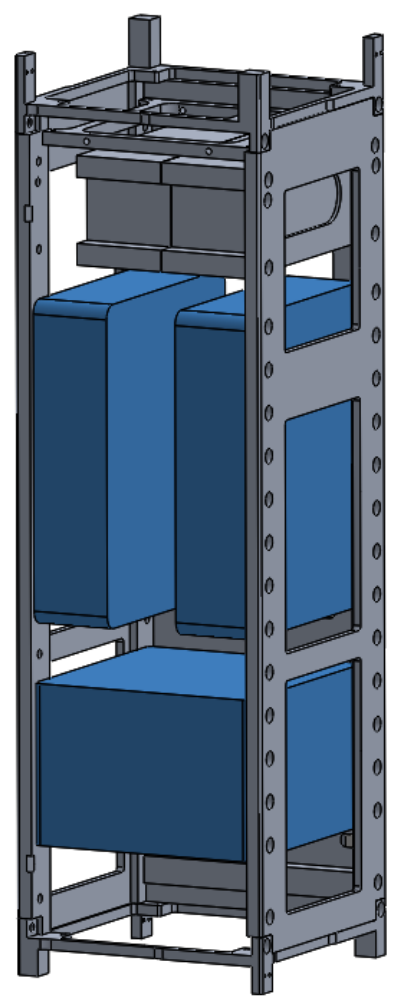

Figure 3.22: CPX can accommodate various payload sizes and mounting configurations. 
The four main panels of the CPX structure were designed to assemble independently. The reason for this is to facilitate access to the internal systems of the CubeSat, without having to disassemble the entire structure. Independent side panels can make troubleshooting less of a hassle, as a single panel can be removed and replaced once repairs have been made, with no real need to completely dismount a payload from the structure (see Figure 3.23).

While the mounting panels are designed to be thick for added stiffness, it should be noted that the remaining two panels are thinner. This was done to compensate for the thick mounting panels in order to maximize the volume allocated to payloads. Most of the internal faces of structure are also flat and free of extruded features in order to facilitate payload integration. However, one main drawback of this design is that the thin walls could be more susceptible to bending moments and buckling during vibration. To get around this issue, while still maintaining the flat internal surfaces of the structure, one option is that the payload enclosure design allow for fastener attachments to its sidewalls. The mounting panel would anchor the payload, and the payload would in turn support the mid-sections of the CPX thin panels.

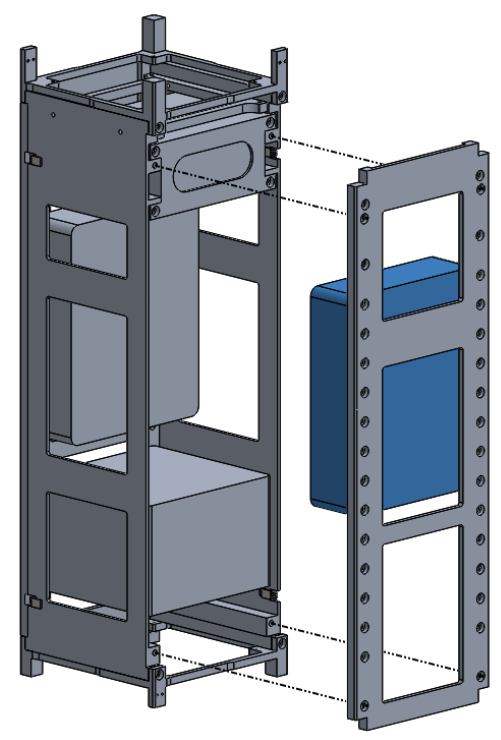

Figure 3.23: Single panels of CPX can be removed for easy access. 
Solar cells can be integrated to supplement power, but the relatively small surface area of CubeSats limits the number of cells that can be installed. To get around this, many structure designs make use of deployable panels (revert to Figure 3.18) for added surface area. However, inherent challenges to such designs include providing adequate protection of the delicate (and expensive) solar cells while in a stowed position, and developing a deployment mechanism with a slim profile that will keep all panels in a compact configuration prior to deployment. Designing slim hinges can be especially challenging, and their mounting location to the CubeSat structure also adds restrictions to the layout of the solar cells. As one possible solution, CPX includes hinges that are placed near the two extremes of the structure and far from the payload compartment to avoid them becoming an obstacle during payload installation (see Figure 3.24). For example, one "leaf" of a sample hinge can be fastened on the inside of the thin side panel, using a shallow recess cutout on the panel to help constrain the in-plane rotation of the hinge. This design approach takes advantage of the panel thickness to minimize the protrusion of the hinge for compact solar panels.

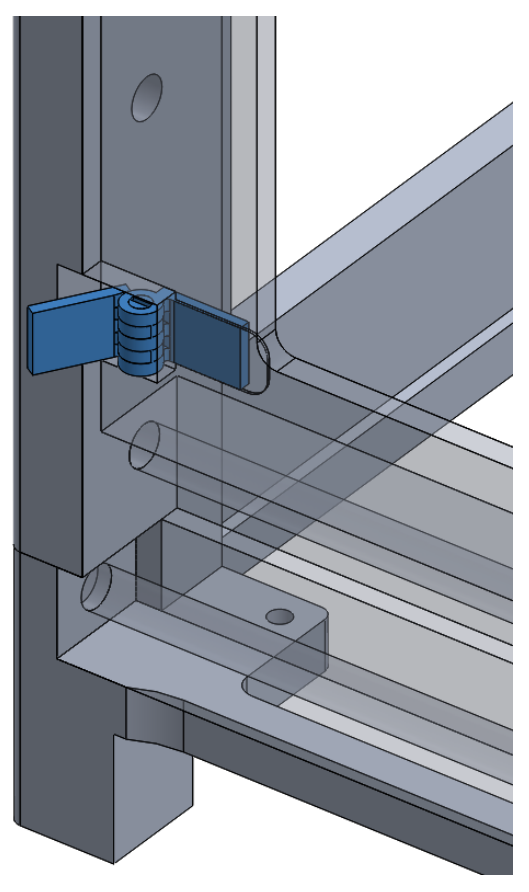

Figure 3.24: Slim-profile hinges sit away from the payload area. 
Batteries are the main source of power for CubeSats, and adequate protection must be provided to avoid accidents that may jeopardize their function. Brackets are used enclose the battery pack, and the design of the inner chamber should allow for sufficient clearance as to not press on the battery (see Figure 3.25). A compressed battery due to poor assembly and/or thermal constriction of the metal bracket can lead batteries to fail and possibly explode.
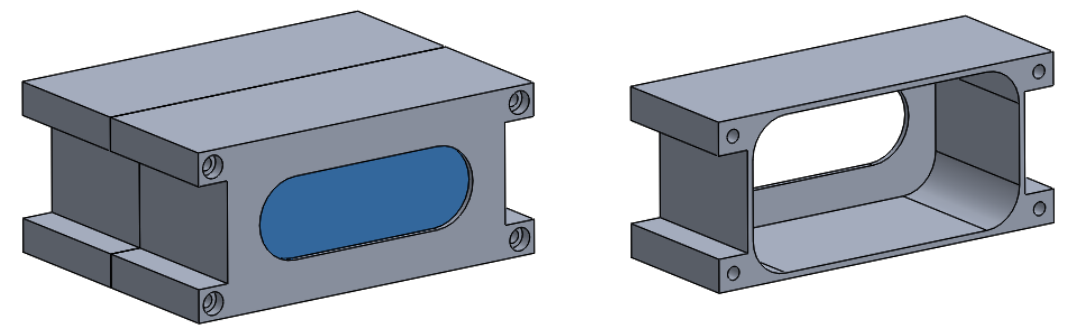

Figure 3.25: CPX battery pack is held together with four fasteners.

With the battery pack assembled, the design of the brackets allows the battery pack to slide securely into guides of the thin panels (see Figure 3.26). In addition, the battery pack only needs to be fastened to one mounting panel, where this design is meant to facilitate overall assembly and troubleshooting. Also note that the hinges sit in the empty space between the guides, and away from the payload area.
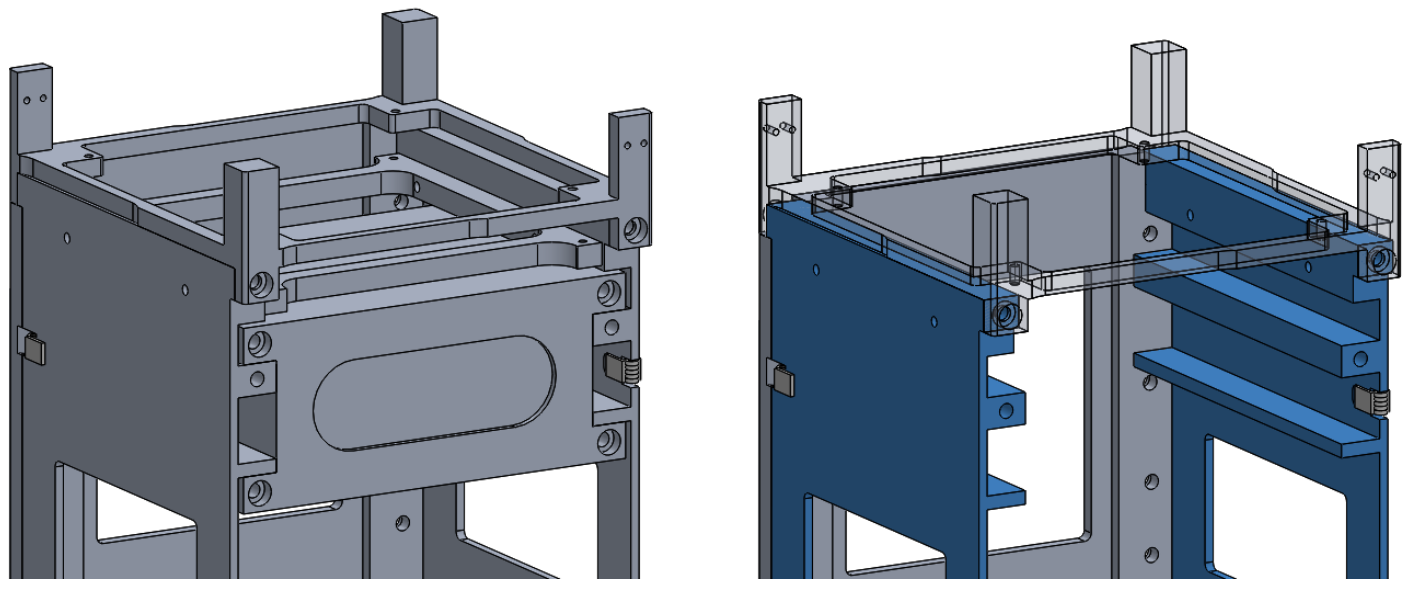

Figure 3.26: Battery pack slides in and bolts to one mounting panel. 


\subsubsection{Assembly}

CubeSat CPX was assembled with the test pod in the same manner described in Section 3.2.4, replacing the mass model for the CPX structure in the CAD assembly (see Figure 3.27). As opposed to the symmetric mass model where the orientation of the z-axis is the same, the CPX structure does not have this symmetry. For this assembly, the battery pack of CPX was chosen to face the cap of the test pod, and the orientation was kept consistent for all FEA modeling.

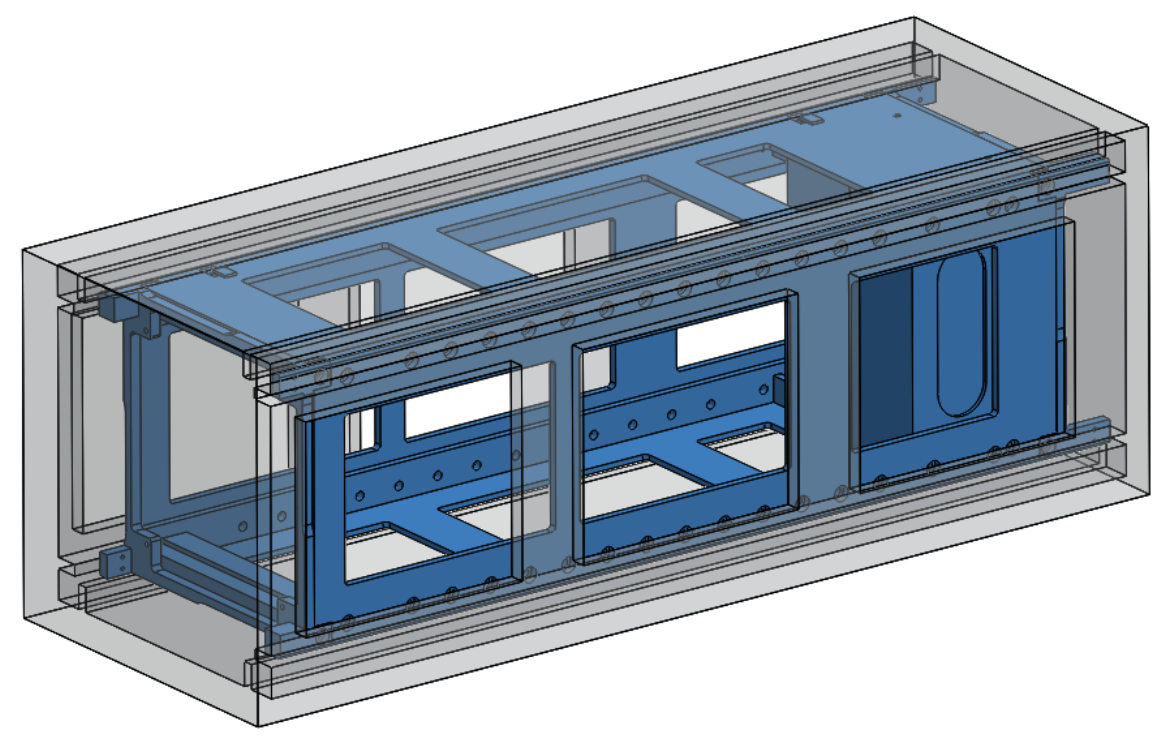

\section{Figure 3.27: CAD assembly of the test pod and CubeSat CPX structure.}

As previously mentioned, the CubeSat design standard requires stand off features at both ends of a CubeSat structure, as these are meant to be the main clamping areas of the satellite for P-Pod, or test pod, integration (see Figure 3.28). The battery pack was chosen to face the cap of the test pod in order to simulate a "worst case" scenario, where the concentrated mass would sit on the large overhang area of the fixture plate assembly (see Figure 3.29). The unbalanced mass distribution with inconsistent support was expected to amplify the dynamic response for analysis. 

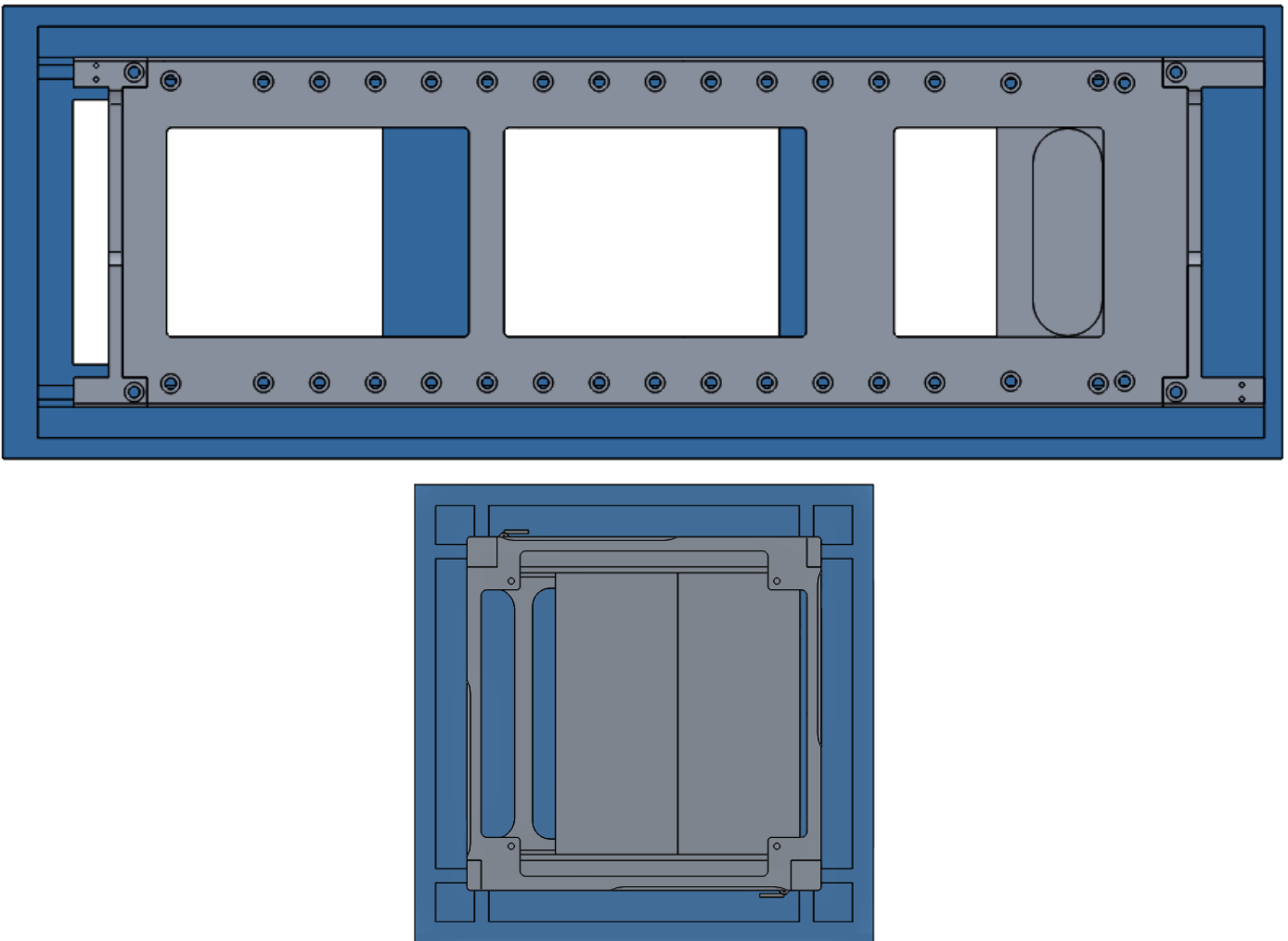

Figure 3.28: Cross-section views of the test pod and CPX assembly.

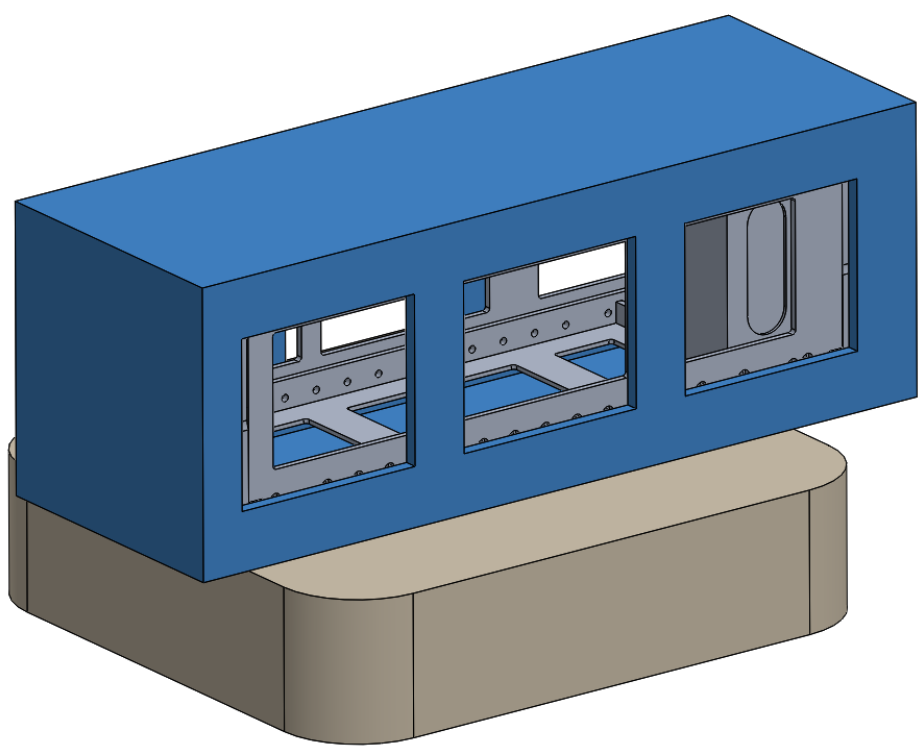

Figure 3.29: CAD assembly of the loaded test pod with the fixture plate. 
Chapter 4

EXPERIMENTAL TESTING, RESULTS, AND DISCUSSION

\subsection{Vibration Table}

All vibrations tests for this experiment were performed using an Unholtz-Dickie Corp. vibration table available at Cal Poly SLO (see Figure 4.1). Also referred to as a shaker or slip table, the vibration table is a robust and precise method of performing dynamic tests, and can be programmed to run vibration profiles including sine sweeps and random vibration. The vibration table is an electro-dynamic system which uses electricity to drive an armature in a linear motion. Vibration isolators built around the moving armature help dampen cross vibrations that may interfere with the system response, and the large steel base of the vibration table helps stabilize the machine during operation. The vibration table model available at Cal Poly SLO can be used in a horizontal or vertical configuration, depending on the type of test to be performed.

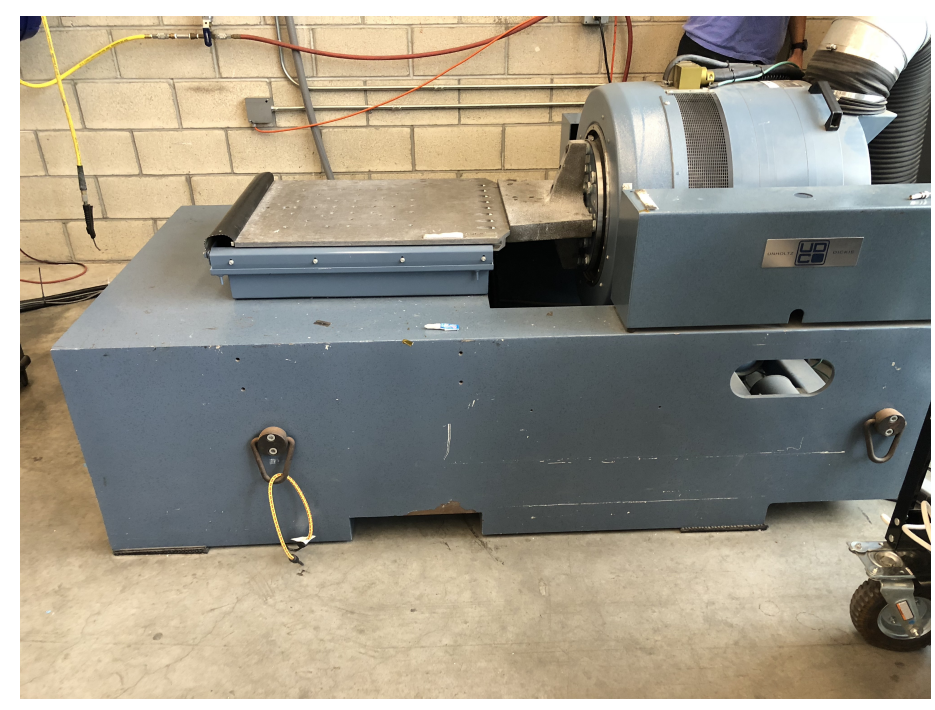

Figure 4.1: Unholtz-Dickie Corp. vibration slip table (horizontal configuration). 
The moving armature is bolted directly to a large slip plate which rests on a lubricated granite slab (see Figure 4.2). The lubricating oil not only minimizes friction, but also "transfers" the stiffness of the granite slab to the slip plate through the adhesion forces of the oil acting between the stationary granite slab and moving slip plate. ${ }^{77}$

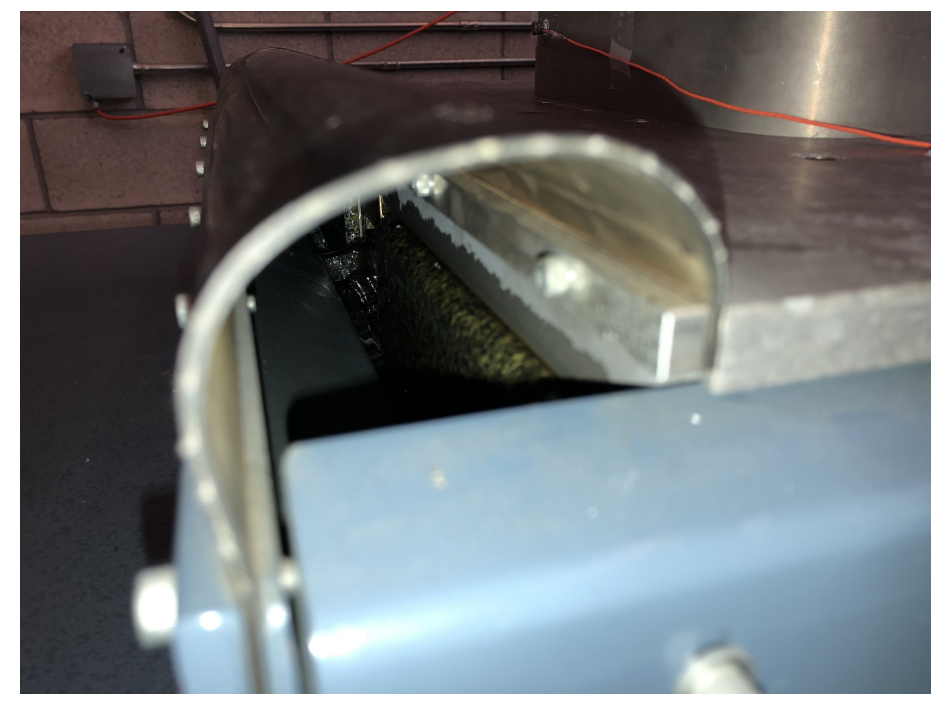

Figure 4.2: Slip plate slides on a granite slab using oil lubrication to minimize friction and improve stiffness.

The control system interface of the vibration table is used to set test parameters and monitor the system response in real time (see Figure 4.3). Important properties like the mass of the test object and other main components in the setup (e.g. fixtures, large hardware, etc.) must be accounted for and entered into the control system, as the program uses this information to calculate the necessary amps needed to drive the armature at the desired acceleration for example. If all the significant masses are not added to the controller, the system cannot compensate for the added weight and may run at a lower than desired input, display an error, or not run at all.

Aside from the mass, other parameters including frequency range, sampling rate, and desired units must be set before running the test. The response data can also be saved to an output file and exported to programs like Matlab or Excel for further 
processing. Operating the vibration table and controller requires special training, and as such, only qualified technicians are allowed to operate the equipment. The CubeSat lab fortunately has qualified technicians, and were most generous in setting time aside to assist in the setup and execution of all experiments performed here.

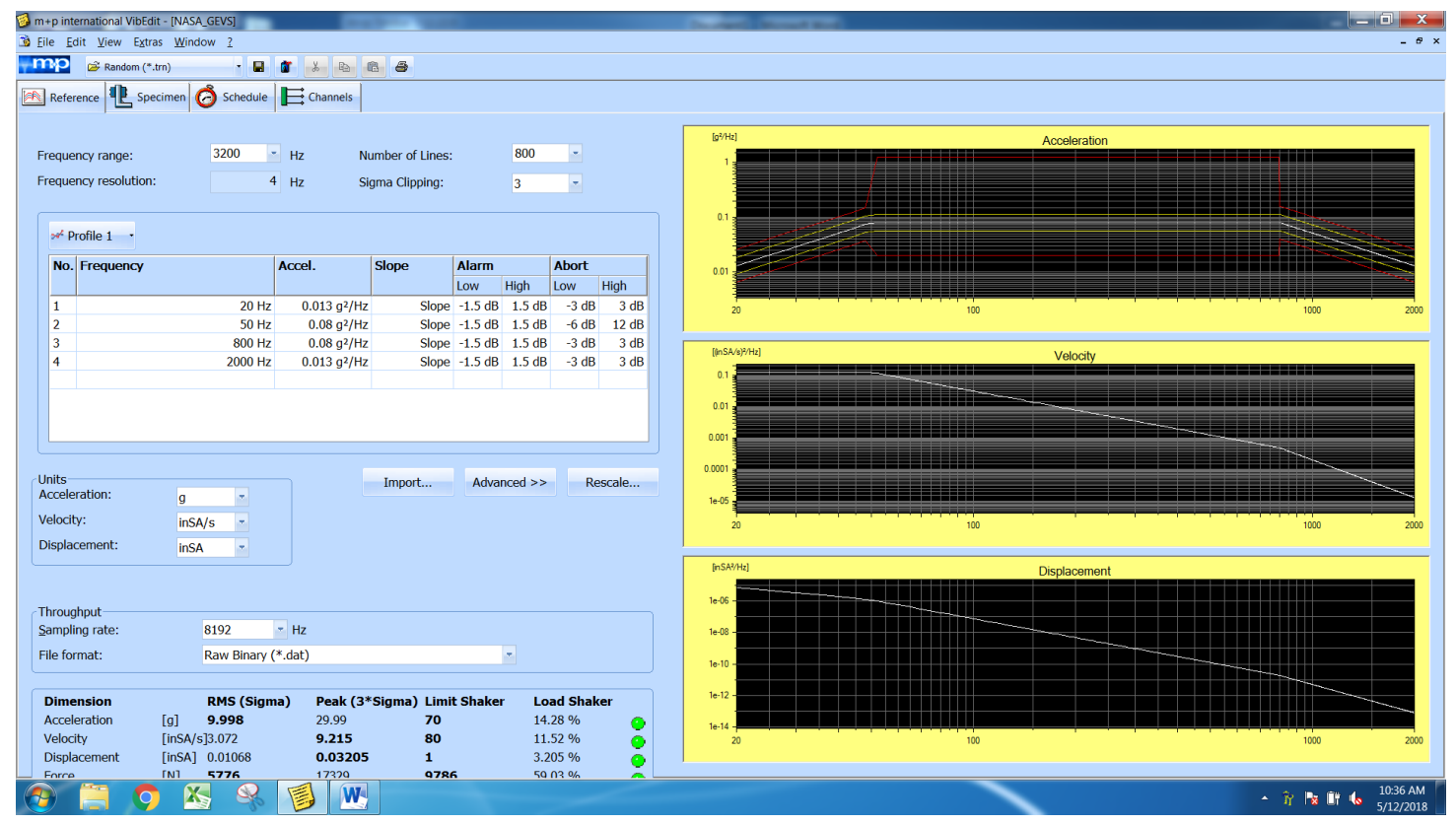

Figure 4.3: Slip table control interface.

In general, dynamic testing should make use of light yet stiff materials, and as was discussed for the fixture plate, magnesium alloy is a material that provides these benefits. This is the reason why the moving components of most vibration tables are made of magnesium, due to the attractive properties of the material. The low weight and high stiffness helps avoid introducing equipment natural frequencies that should not be pronounced in system response of the test object. Dynamic test equipment like vibration tables are designed to have minimal effects on the system response, and using materials like magnesium is one of the techniques used to achieve this. The test setup assembly is also important, as the more components that sit between the test object and the slip table, the more degrees of freedom (and weight) that are 
available to cause noise and throw off the accuracy of the response. For example, if bolts on the fixture plate were to become loose during a vibrations test, the stiffness of the system would change, affecting the system response. Now suppose that an additional "bracket" was needed to attach the test pod to the fixture and these bolts were torqued incorrectly. This would now amplify the error caused by the loose bolts of the fixture. So, a simple yet rigid setup should be used when possible. 


\subsection{Measurement Hardware}

To measure the dynamic response of a vibrating system, measuring instruments need to be integrated into the setup in order to monitor the behavior of the system. Aside from monitoring the test object, it is good practice to also have a reference measurement to verify the desired input to the system. For non-contact measurements, a laser Doppler vibrometer (LDV) uses reflected beams off the surface of the vibrating object to measure the amplitude and frequency response of the system. For contact measurements, an accelerometer can be attached directly to the test object to measure the acceleration of the system response (see Figure 4.4), and are available in single or triaxial models. Using an LDV over an accelerometer has benefits such as contact not being required, measuring objects with restricted access, and objects that are too small, hot, or cold for physical attachments. ${ }^{47}$ However, a drawback of LDV's is their relatively high cost (compared to accelerometers), but the benefits can outweigh the cost based on the application intended. Benefits of accelerometers include their durability, ease of installation, accuracy, long life span, and large dynamic range. ${ }^{72}$ For all experiments here, $\mathrm{PCB}$ accelerometers were used to gather all response data.

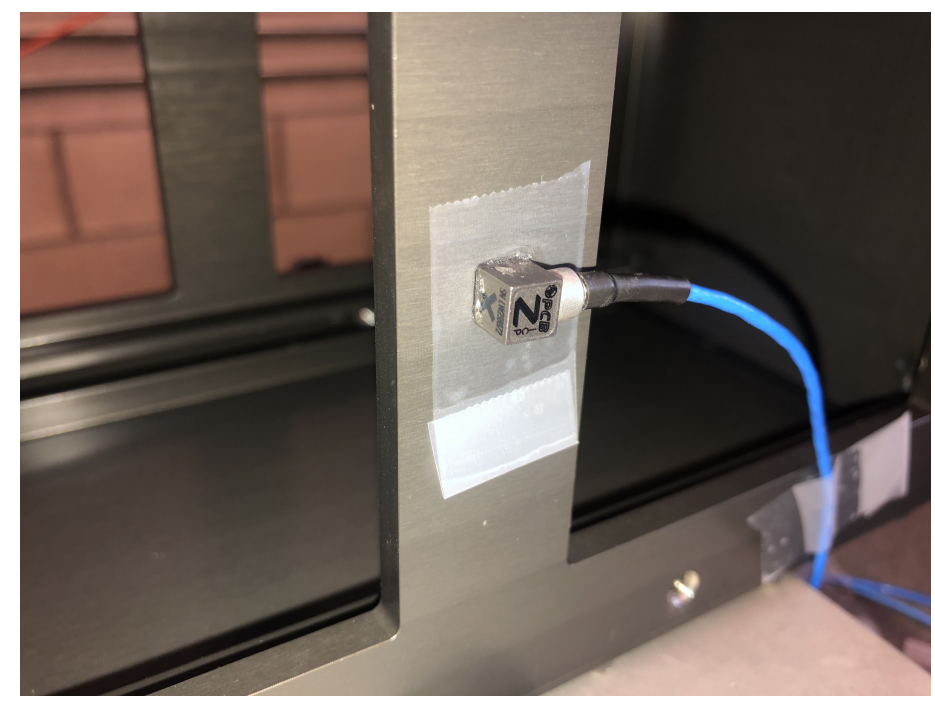

Figure 4.4: Tri-axial accelerometer measures the dynamic response. 
In principal, an accelerometer functions as a single degree of freedom (1-DOF) springmass-damper system, where a mass $m$ applies a stress to a bed of piezoelectric (PE) material due to the acceleration $a$ acting on the accelerometer (see Figure 4.5). The PE material then generates a voltage that is proportional to the applied acceleration, ${ }^{16}$ and the voltage is then translated to read as acceleration on the system controller interface. As any other structure, accelerometers each have their own natural frequencies that are inherent by design. This fact should be considered when setting up a dynamic test since the size, mounting location, and orientation of the accelerometer may affect the accuracy of the system response.

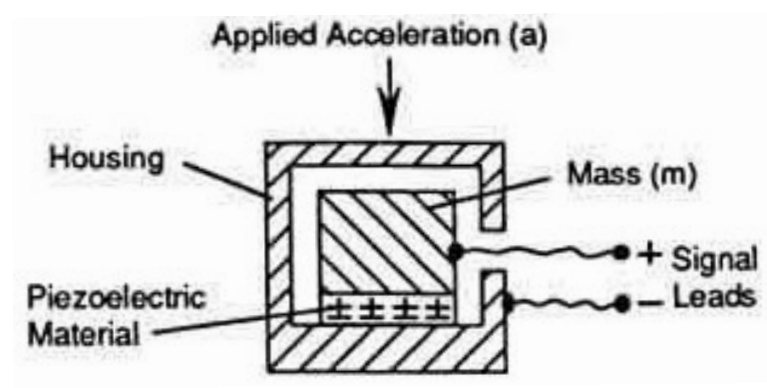

Figure 4.5: Basic internal structure of a typical accelerometer. ${ }^{15}$

Mounting of an accelerometer is important when setting up a vibrations test, as this determines the "stiffness" of the accelerometer and how well it can read the dynamic response of the system. The more rigid the accelerometer is attached to the vibrating object, the better it can pick up on the details of the response (see Figure 4.6). Several mounting options are available, with some accelerometer models designed to be used with specific mounting attachments. The type of mounting will depend on the type of accelerometer used, the level of precision needed, and whether or not the mounting surface is to remain untarnished after testing. A stud mount is the ideal mounting method, as the accelerometer is bolted directly to the vibrating object for maximum response sensitivity. However, the drawback of using this method is that a stud must be drilled into the test object, which is not suitable for many applications. 


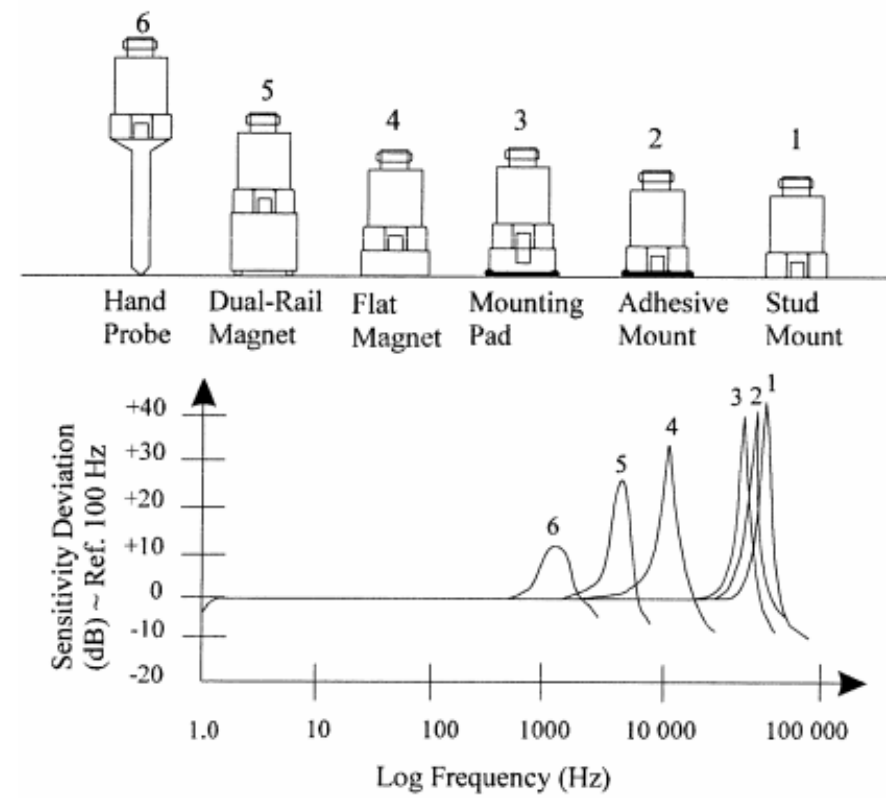

Figure 4.6: Sensitivity of accelerometer using different mounting methods. ${ }^{52}$

As seen in Figure 4.6, hand probing is the least invasive method of mounting an accelerometer, but this method sacrifices the sensitivity and accuracy of the response. Also note that although stud mounts are the most sensitive, adhesive mounts and mounting pads have similar sensitivities and yet are non-destructive forms of mounting. Again, the type of mounting selected will depend on the application and accuracy desired (see Appendix C for more details).

For all experiments here, an adhesive mount method was used to attach all accelerometers of the vibration test setup. As property of the CubeSat lab, the test pod, mass model, and fixture plate are used in different types of testing and analysis not limited to vibrations. So it was imperative that these structures remain unaltered throughout the course of these experiments. 


\subsection{Assembly}

\subsubsection{Test Pod Configuration}

Two assembly configurations were used to gather data for the dynamic response of the test pod and mass model system. In the first configuration, testing was performed with the test pod alone (see Figure 4.7), and the second configuration was tested with the mass model loaded in test pod. It was necessary to analyze the behavior of the empty test pod first, as this information was essential in validating the results of the FEM models.
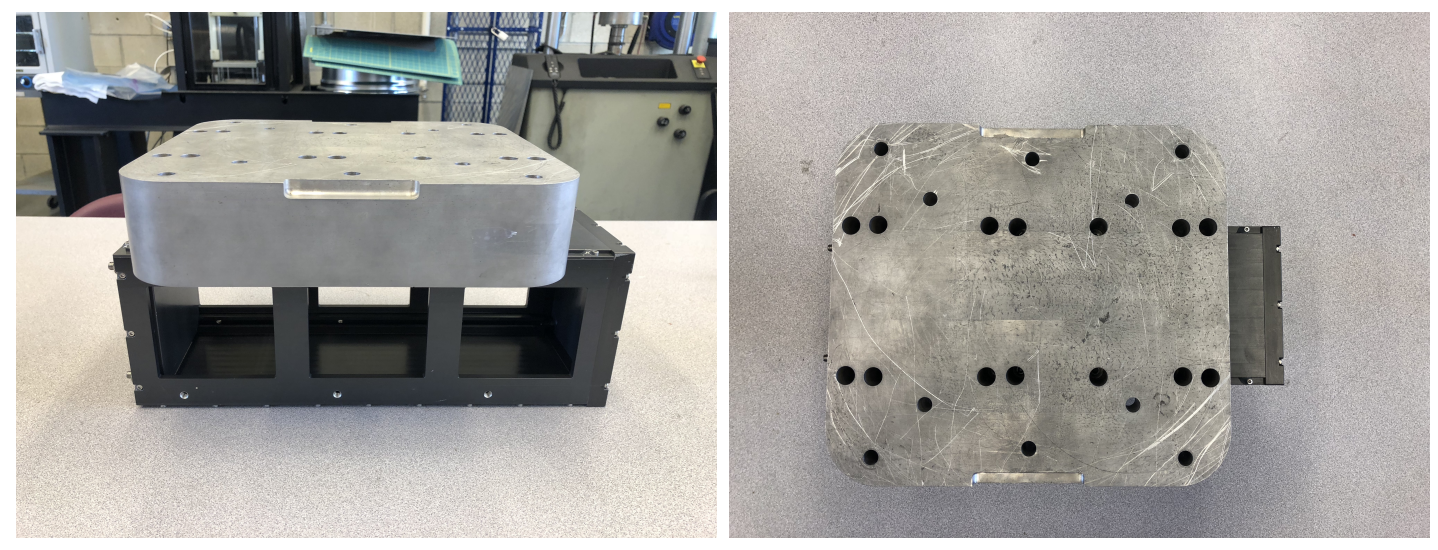

Figure 4.7: The test pod is bolted thru underside of the fixture plate.

Before mounting the test pod to the fixture plate, all fasteners and staking of the test pod were inspected. The screws that hold down the cap of the test pod were inspected and torqued to the $1.13 \mathrm{~N}-\mathrm{m}(10 \mathrm{in}-\mathrm{lbf})^{22}$ specification to ensure that the lid was securely held in place. The spring plungers that press on the internal floating plate of the test pod were also adjusted to prevent the floating plate from rattling. Finally, the jam nuts on the spring plungers were also torqued to the specification of $3.39 \mathrm{~N}-\mathrm{m}$ (30 in-lbf $)^{22}$ to keep the spring plungers from loosening during the test. 
The test pod was bolted to the fixture plate using the 6-bolt pattern, which can only be accessed from the underside of the fixture plate as seen in Figure 4.7. Six hex-socket screws (see Figure 4.8) were each torqued to the specification of $2.6 \mathrm{~N}-\mathrm{m}$ (23 in-lbf) ${ }^{22}$ which secured the test pod to the fixture plate. No staking was applied to the six hex-socket screws, as it is difficult to clean off

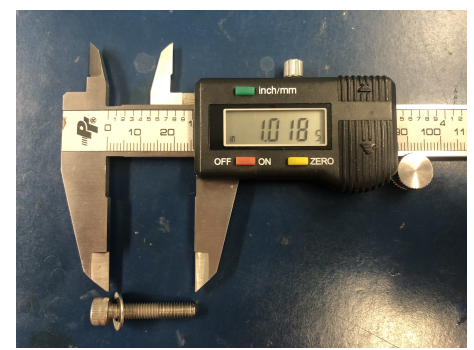

Figure 4.8: Small screws used for the test pod assembly. staking in the deep bores of the fixture plate.

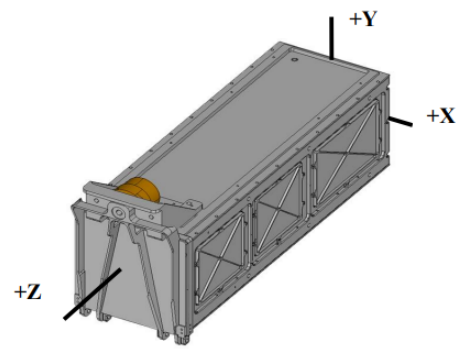

Figure 4.9: P-Pod axes. $^{22}$

The mass and major dimensions of the test pod and fixture plate were recorded prior to assembly, as these parameters were needed to build the CAD models. Based on the CubeSat standard, the z-axis of a CubeSat is defined along the longitudinal (length of body) axis of the satellite, and this same orientation standard applies to P-Pods and test pods (see Figure 4.9). For these experiments, the z-axis was chosen as the primary axis of interest for the dynamic response of the system. With the test pod fully assembled, it was ready for vibration table integration (see Figure 4.10).

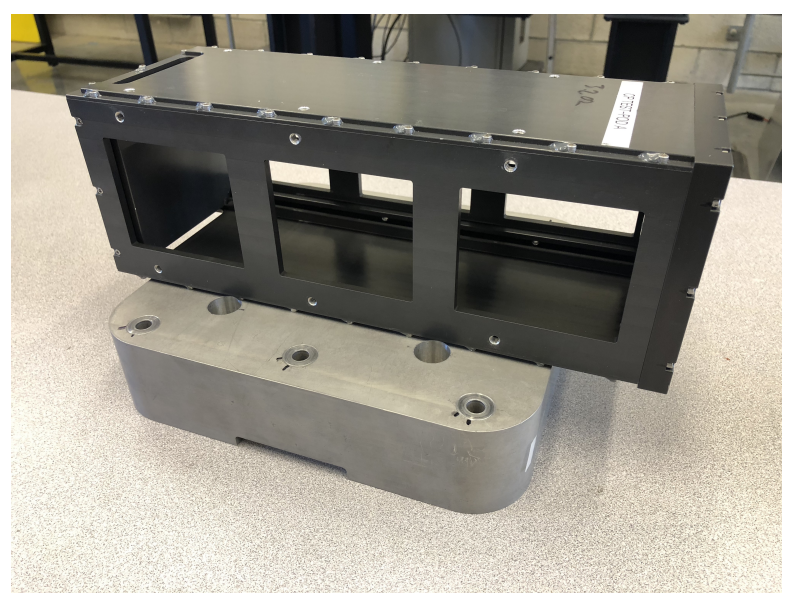

Figure 4.10: Bolted test pod assembly. 


\subsubsection{Test Pod with Mass Model Configuration}

For the second assembly configuration, the mass model was integrated with the test pod (see Figure 4.11) to compare the dynamic response of the system with and without the loaded mass model. Like the first configuration, the longitudinal z-axis was the primary axis of concern for this setup. Testing in the z-axis direction is important as CubeSats have more degrees of freedom available for movement, as opposed to the more restricted $\mathrm{x}$ and $\mathrm{y}$ directions. Furthermore, since much of the sensitive equipment of CubeSats (e.g. thin circuit boards) are assembled in the $\mathrm{x}-\mathrm{y}$ plane of the structure, such equipment is more susceptible to damage from transverse loading in the z direction (see Appendix A.7 for more details). As such, CubeSats are carefully inspected before and after testing to ensure components are still intact, and to verify the CubeSat system still functions as intended.

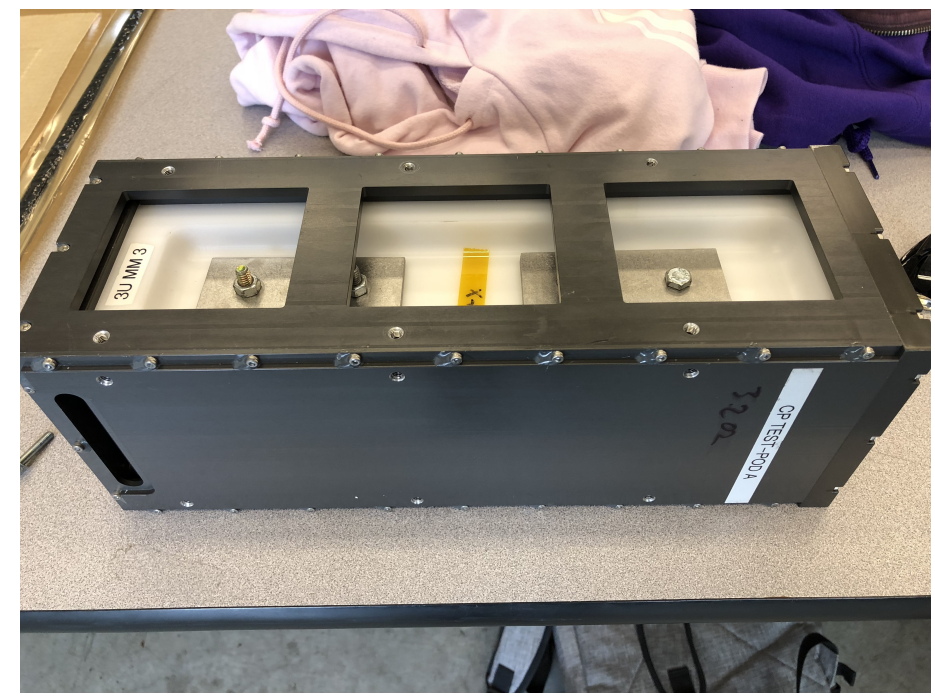

Figure 4.11: Test pod cap is carefully replaced once the mass model has been loaded.

The mass and main dimensions of the mass model were recorded prior to test pod integration, as this information was needed for the CAD models. With the mass model loaded in the test pod, it was necessary to torque the cap screws to the specification 
of $1.13 \mathrm{~N}-\mathrm{m}$ (10 in-lbf). ${ }^{22}$ This is critical in the assembly, as the cap bears the inertia of the mass model during the vibration test and keeps the mass model from being ejected, especially when testing in the z direction.

Another key part of the mass model integration was the adjustment of the spring plungers. With the loaded mass model sitting against the floating plate of the test pod, a sufficient clamping force needs to be applied to the mass model to keep it as rigid as possible during the vibration test. A procedure is defined in the test pod user guide for proper spring plunger adjustment of a loaded test pod, but since a less fragile CubeSat substitute was being used, the spring plungers were adjusted to bottom out, as permitted by the test pod user guide. ${ }^{22}$ Viewing windows are available on the test pod and were used to monitor the adjustment of all the spring plungers (see Figure 4.12). The loaded test pod was then bolted to the fixture plate in the exact same manner discussed in Section 4.3.1.

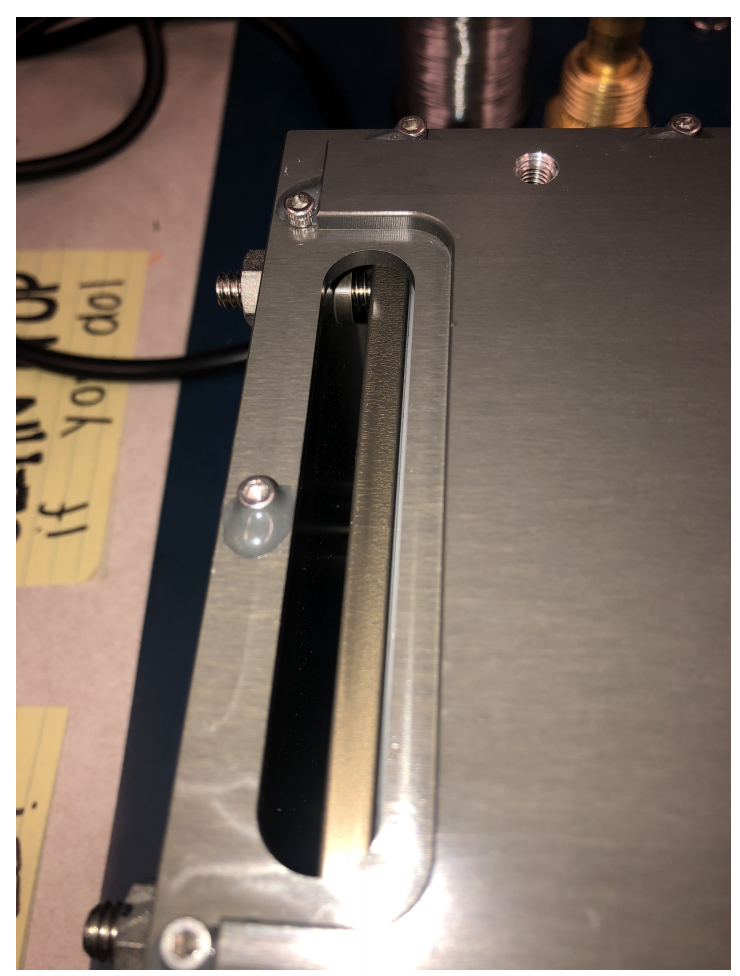

Figure 4.12: Spring plungers are adjusted once the mass model is loaded. 
With the loaded test pod assembled, it was ready for vibration table integration. The slip plate of the vibration table has various bolt patterns to accommodate test objects of different sizes, and this is one of the main appeals of using vibration tables for dynamic testing. The fixture plate was designed around one of the bolt patterns that centers the fixture plate with respect to the slip table, as

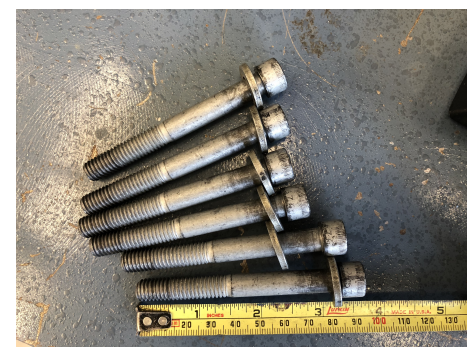

Figure 4.13: Large bolts used for the fixture assembly. this aligns the fixture with the line of action of the reciprocating armature during testing. Six large hex-socket bolts (see Figure 4.13) were used to clamp the fixture down to the slip plate, and they were torqued to the specification of $21.7 \mathrm{~N}-\mathrm{m}$ (16 ft-lbf). ${ }^{22}$ No staking was applied to the six large bolts, as these tests were performed for modeling purposes only.

The z-axis of the test pod and mass model configuration can be flipped in either direction, as long as the axis aligns with motion of the slip table. However, the orientation of the setup was kept consistent by letting the test pod cap face toward the armature of the vibration table in all tests performed (see Figure 4.14).

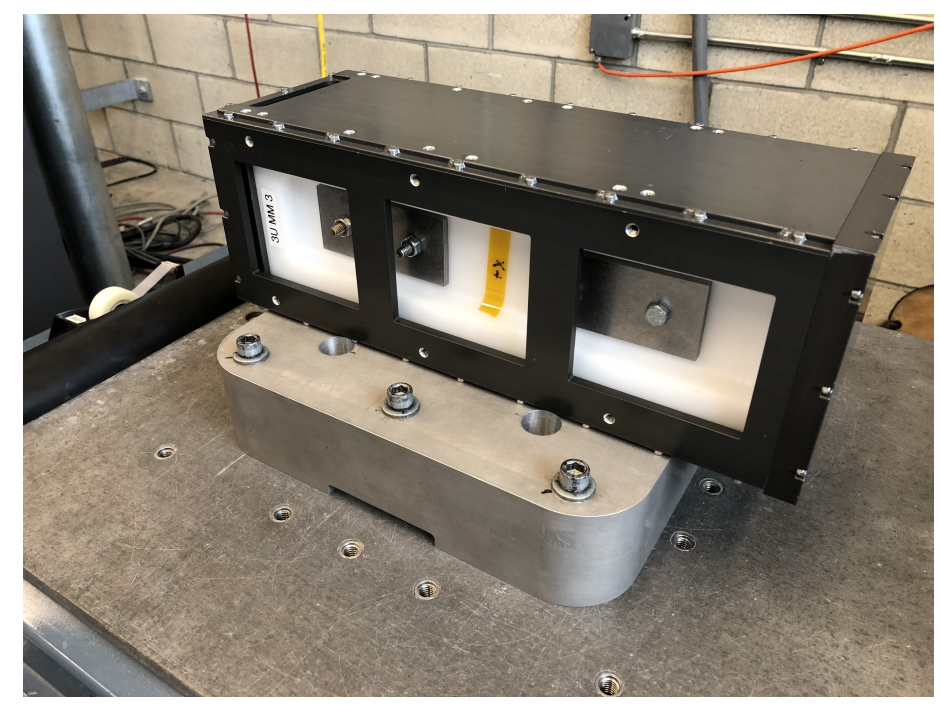

Figure 4.14: Bolted test pod and mass model assembly. 


\subsection{Testing}

\subsubsection{Dry Run (fixture plate)}

The main objective of these experiments was to analyze the system response and identify the natural frequencies of the test pod and mass model. As previously mentioned, every component used in a vibration test setup will each have their own unique set of natural frequencies, and the combination of these frequencies influence the response of the system as a whole. For this reason, testing was done using a "bottom-up" approach where the fixture plate was tested first in a dry run (see Figure 4.15), followed by testing with the test pod attached, and finally with the mass model loaded in the test pod.
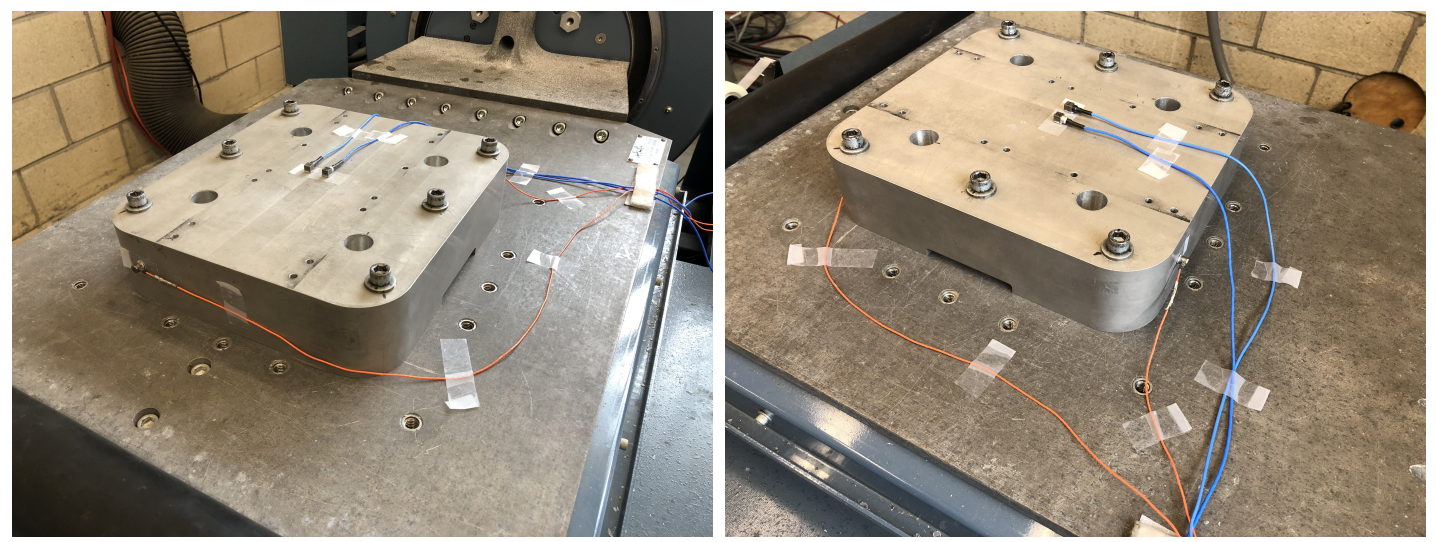

Figure 4.15: Triaxial (blue) and single-axis control (orange) accelerometers are attached to taped surfaces using an adhesive.

Two types of vibration tests, sine sweep and random vibration, were performed on all setups. In a sine sweep test, the controller "sweeps" through a specified range of frequencies and displays peaks in the system response as indicators of a natural frequency (revert to Section 2.1.1 on resonance). For all the sine sweep tests performed here, the frequency was set to sweep from 20 to $2000 \mathrm{~Hz}$, with a sweep rate of 3 oct/min, and a 2-minute duration for each test, as recommended by NASA GEVS. ${ }^{40}$ 
These are standard parameters that are used to qualify CubeSats, and as such, the same levels were applied in these tests.

For random vibration tests, a random input profile must be programmed and the controller then generates a signal which excites many frequencies of the system, all at the same time over the specified frequency range. This results in the simultaneous excitation of natural frequencies pertaining to different components in the system, as opposed to sine sweeps where each resonance frequency is excited separately. ${ }^{60}$ NASA GEVS specifies the acceleration spectral density (ASD) levels and frequency range that should be used for random vibration testing (see Table 4.1), and notes different profiles based on the mass of the object being tested. CubeSats are qualified using these levels, and so these same levels were applied to all random tests performed here for 1 minute using the $22.7 \mathrm{~kg}$ (50 lb) profile, as seen in the plot of Table 4.1.

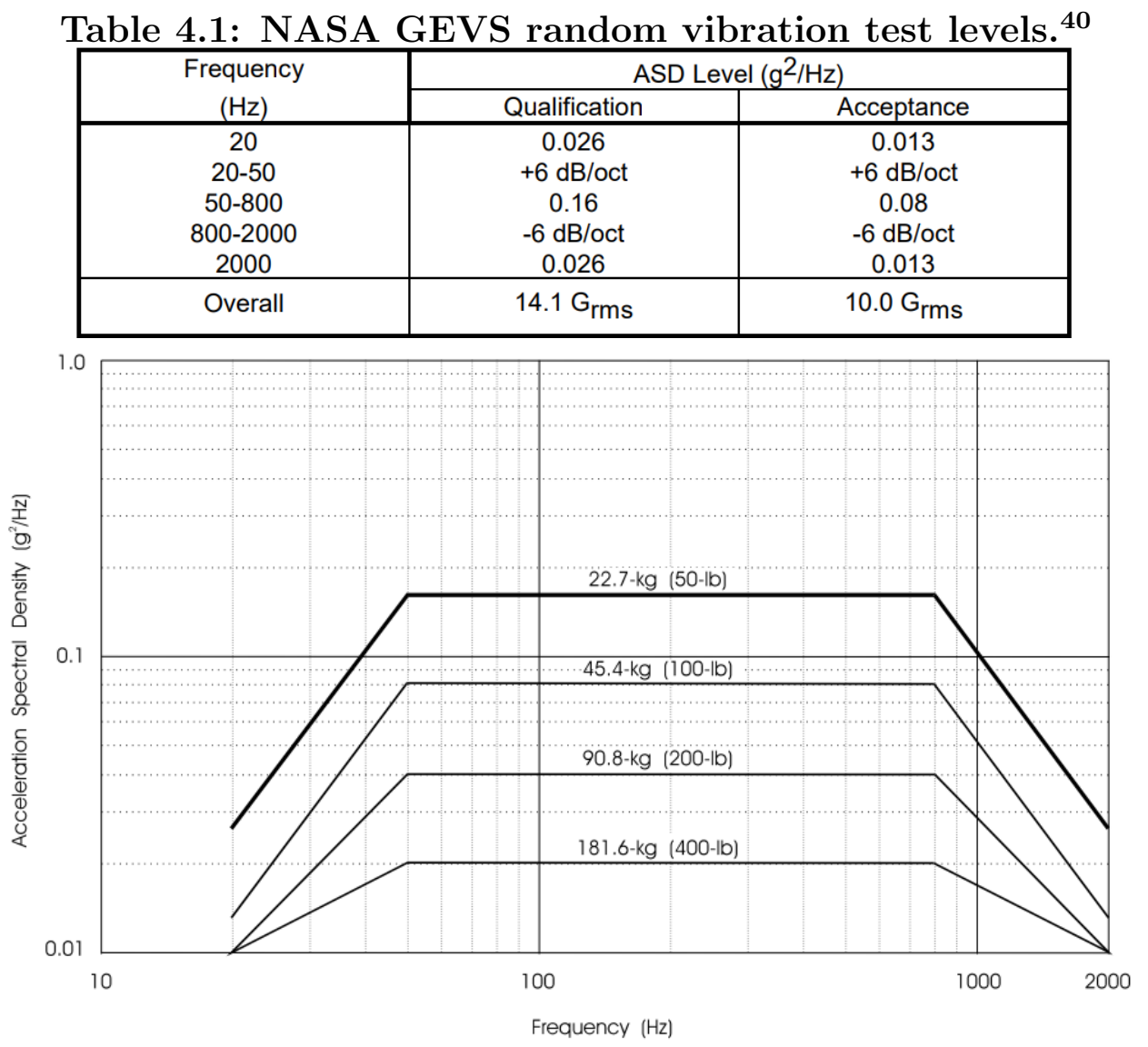




\subsubsection{Sine Sweep}

Using an input of $0.5 \mathrm{~g}\left(\mathrm{~m} / \mathrm{s}^{2}\right)$, the sine sweep test showed a slight peak in the system response at approximately $1300 \mathrm{~Hz}$ (see Figure 4.16). Beyond this point, the triaxial accelerometers begin to diverge simultaneously, while the control accelerometers remain fairly constant throughout most of the response. Hence, the control accelerometers helped verify the $0.5 \mathrm{~g}$ input to the system. From previous experiments performed using this vibration table, a system response is usually observed between 1000 and $1500 \mathrm{~Hz}$, which suggests that this is an inherent natural frequency of the vibration table machine. Using this setup, noise has also been shown to be pronounced beyond $1500 \mathrm{~Hz}$, which may help explain why the triaxial accelerometers diverge after this point. While a peak is pronounced in the response of Figure 4.16, the magnitude is not sufficient to cause concern. Extensive work has already been done on the fixture design, with its first natural frequency occurring beyond the 2000 $\mathrm{Hz}$ range. So, the fixture is not the sole culprit of the peak in the response.

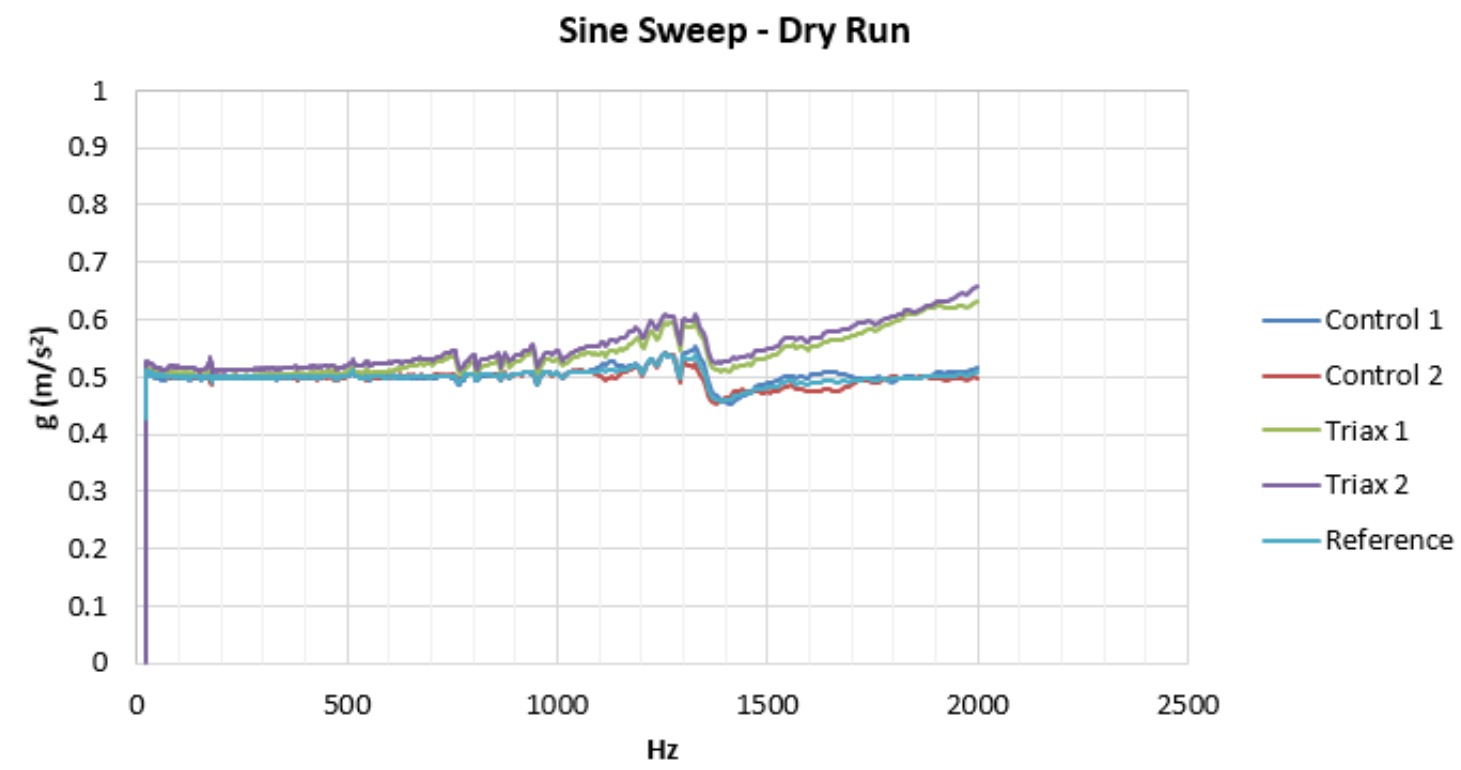

Figure 4.16: Triaxial and control accelerometers verify the $0.5 \mathrm{~g}$ input. The reference pertains to the feedback from the vibration table controller. 


\subsubsection{Random Vibration}

Using the NASA GEVS test levels suggested in Table 4.1, the random vibration test showed that all responses fall within the limit set by the specification (see Figure 4.17). Both triaxial accelerometers had more pronounced responses in the z-axis, but with no significant peaks. All other axes had much less pronounced responses that fell well below the NASA GEVS limit, showing that the vibration was not as intense in the transverse directions, which is expected for the relatively rigid fixture plate. So from the sine sweep and random vibration data, it was confirmed that the fixture plate has no significant natural frequencies below $2000 \mathrm{~Hz}$ that may influence the response of the test pod and mass model, which is the ideal case.

The plots of the two control accelerometers were omitted from Figure 4.17 to reduce clutter in the plot, but were verified nonetheless and showed to closely match the reference data from the vibration table controller.

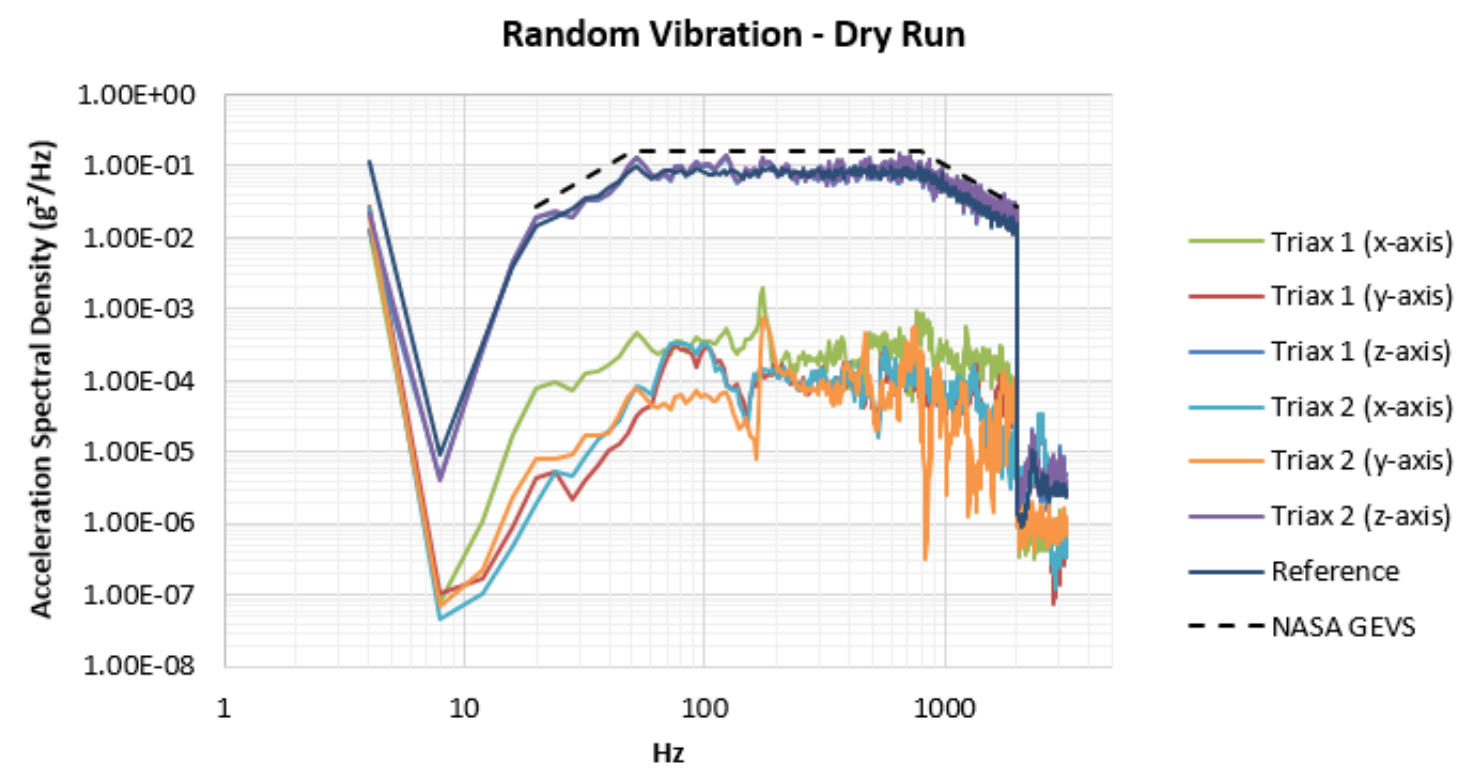

Figure 4.17: All fixture plate responses fall within NASA GEVS random vibration limits. 


\subsubsection{Test Pod}

For the test pod assembly, two sine sweeps were performed along with an intermediate random vibration test. This is standard practice in CubeSat testing to detect if any significant changes have occurred in the system response after the more violent random vibration test has been performed. Ideally the system response should stay relatively the same, but significant irregularities in the second sine sweep can indicate that something has changed in the system or setup, such as loosening of fasteners or plastic deformation in the setup, for example. In such cases, the setup would need to be inspected, troubleshooted, and retested as necessary for qualification.

For these tests, the two single-axis control accelerometers were attached to the fixture plate at the same locations as the dry run, and triaxial accelerometers were attached to a "beam" and cap of the test pod (see Figure 4.18). All surfaces were taped before attaching accelerometers with the use of an adhesive, as the tape protects the surfaces and facilitates clean up. Tape was also used to tie down accelerometer lead cables, as these cables are sensitive to movement and can affect the system response.

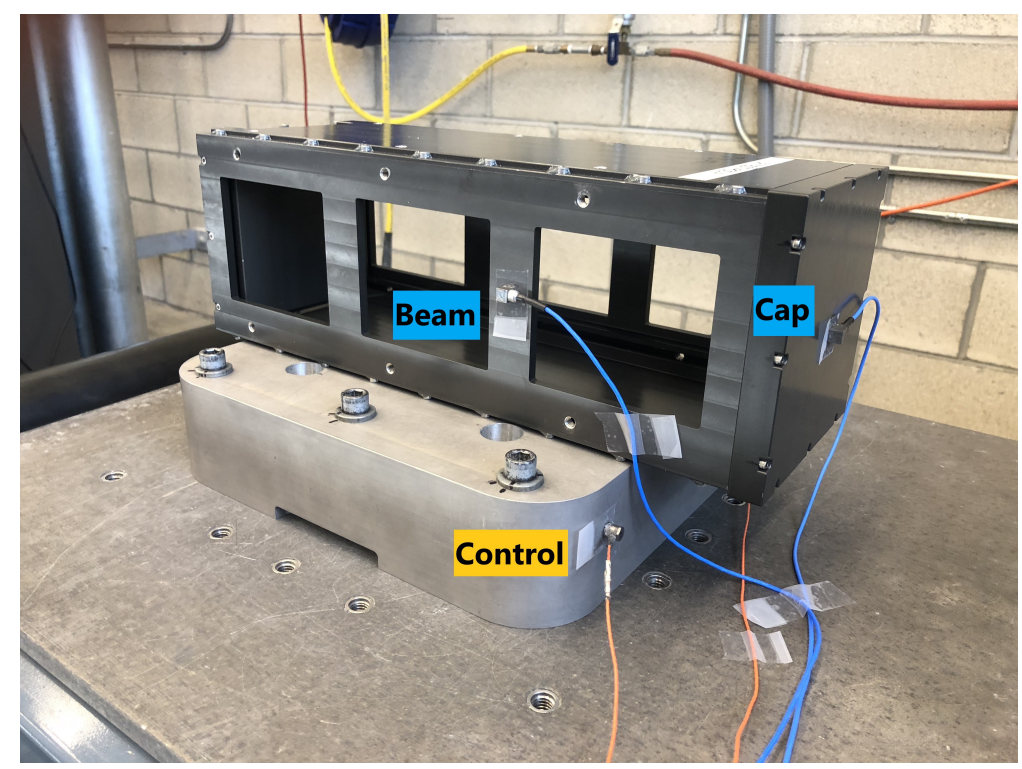

Figure 4.18: Triaxial accelerometers on the beam and cap of the test pod. 


\subsubsection{Pre Sine Sweep}

Using a $0.5 \mathrm{~g}$ input for the first sine sweep test, the response had prominent peaks at approximately 550, 1120, and $1810 \mathrm{~Hz}$ (see Figure 4.19). Upon closer inspection of the largest peak at $550 \mathrm{~Hz}$, note that two different peaks can be identified that are close to each other. This may be in part due to the effects of anti-resonance, which is the destructive interference between a driving force (e.g. vibration table) and another oscillating body (e.g. test pod). The largest peak corresponds to approximately 10 g, which is roughly 16 times the largest response observed for the fixture plate alone. This may be due to the thin hollow-box nature of the test pod, which makes it more susceptible to displacements (see Appendix A.7 for more details). Up to the 1500 $\mathrm{Hz}$ range, note that the beam has slightly more prominent peaks as opposed to the cap of the test pod. Beyond $1500 \mathrm{~Hz}$ however, the response of the beam dies down yet the cap response increases by more than one order of magnitude, possibly due to transverse loading and the increased noise levels generally observed in this range.

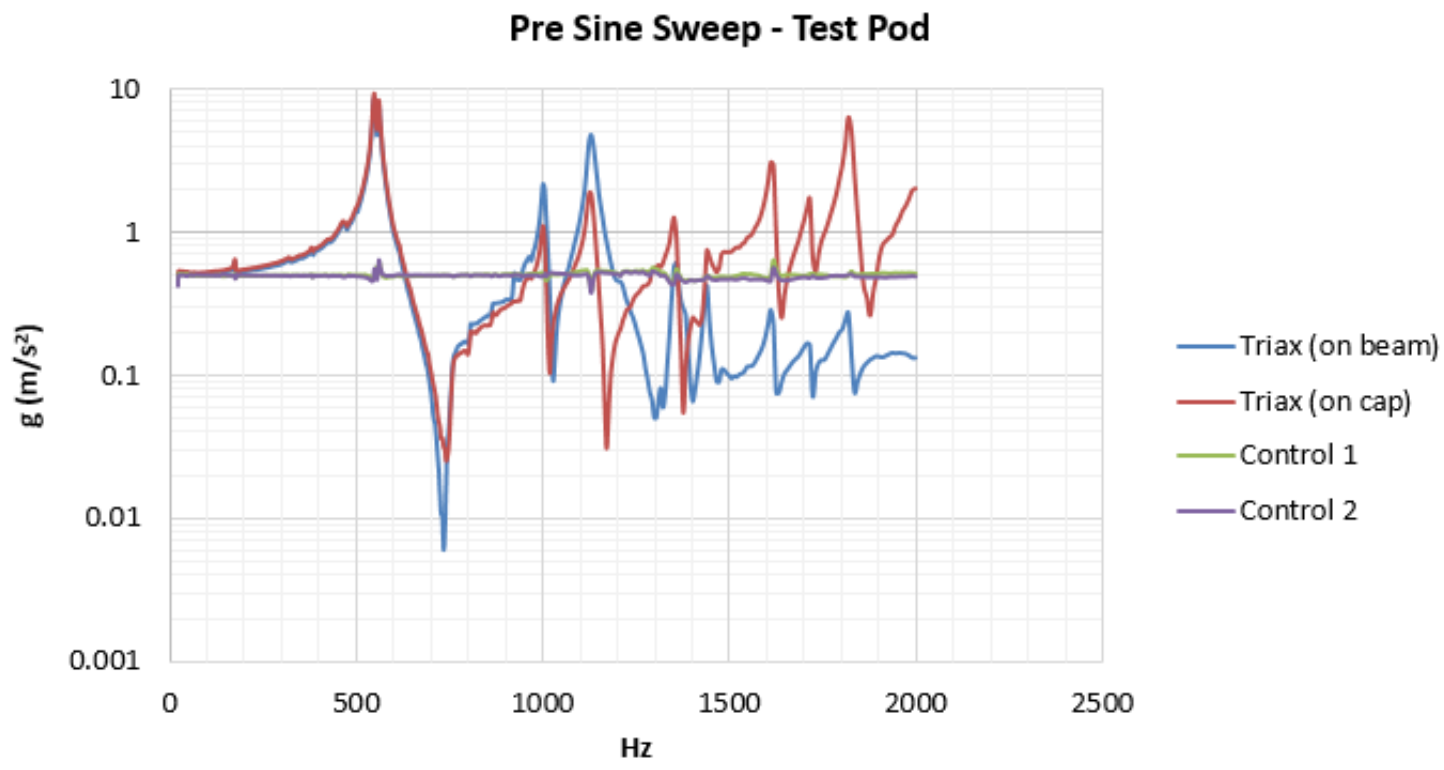

Figure 4.19: Test pod has as the largest response at approximately 550 $\mathrm{Hz}$, and control accelerometers verify the $0.5 \mathrm{~g}$ input. 


\subsubsection{Random Vibration}

The response of the test pod stays within the NASA GEVS limit up to approximately $400 \mathrm{~Hz}$, at which point all responses begin to surpass the GEVS baseline with peaks of significant magnitude (see Figure 4.20). Note that two prominent peaks can be identified in the response, corresponding to approximately $520 \mathrm{~Hz}$ and $1100 \mathrm{~Hz}$. These two peaks closely correspond to the first two peaks of the previous sine sweep test, which is further evidence that the test pod has natural frequencies at these two points. Also note that the response becomes more chaotic beyond the $1200 \mathrm{~Hz}$ range, possibly due the increased levels of noise in this range. While the response in all axes exceeds the NASA GEVS baseline after $400 \mathrm{~Hz}$, the standard does provide a plus/minus tolerance profile (not shown) for qualification. While the responses are relatively high, they are not of real concern since the test pod is meant to function with a loaded CubeSat, and not on its own.

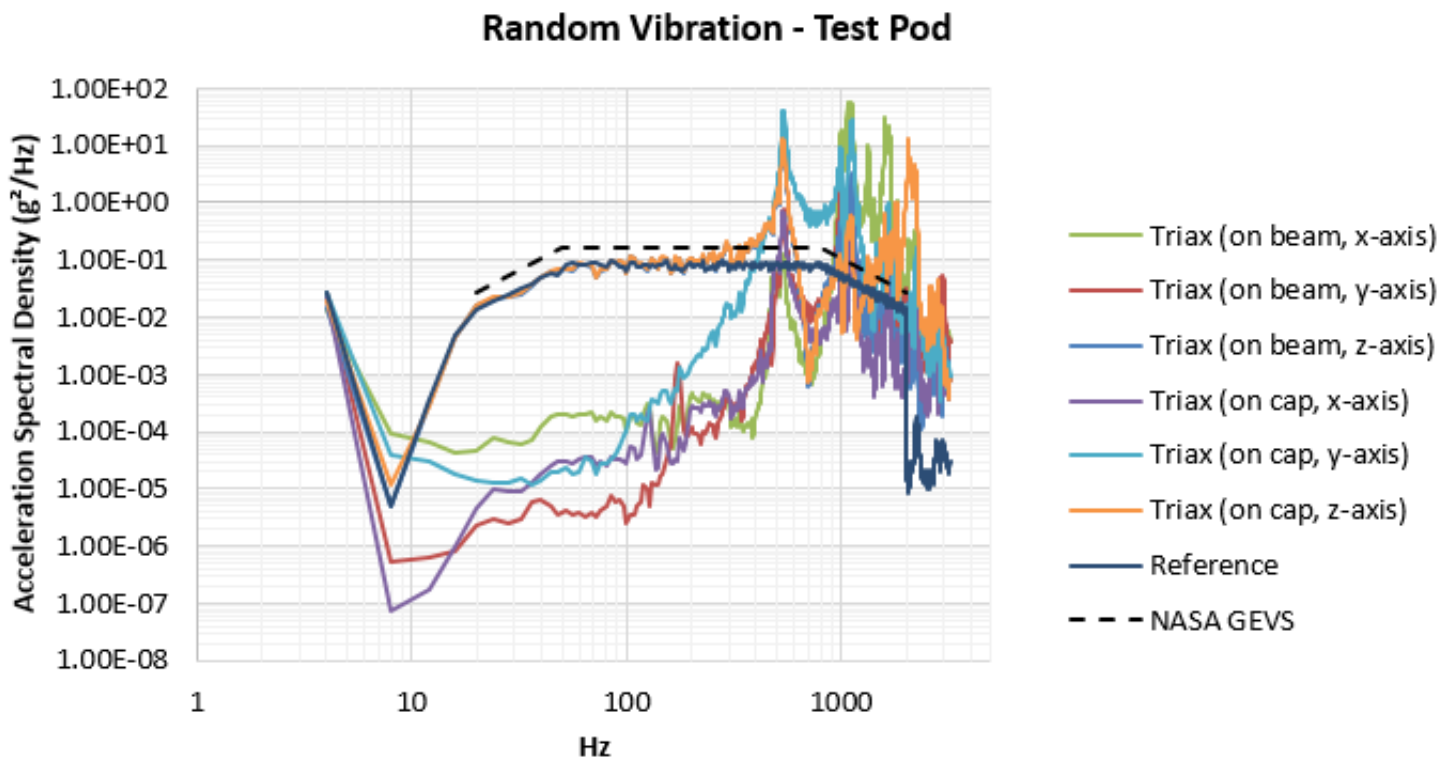

Figure 4.20: Random vibration of test pod has a peak response at $520 \mathrm{~Hz}$ and $1100 \mathrm{~Hz}$. 


\subsubsection{Post Sine Sweep}

Compared to the pre sine sweep data, the post sine sweep response for the test pod had no significant irregularities following the random vibration test. The two prominent peaks still correspond to $550 \mathrm{~Hz}$ and $1120 \mathrm{~Hz}$, just as before (see Figure 4.21). The peak amplitudes also stayed relatively the same, indicating no real changes occurred to the system or setup during the random vibration test. These were positive results, as this is concrete evidence that the test pod has natural frequencies at approximately $550 \mathrm{~Hz}$ and $1120 \mathrm{~Hz}$. The controls also verified the $0.5 \mathrm{~g}$ input to the vibration table controller.

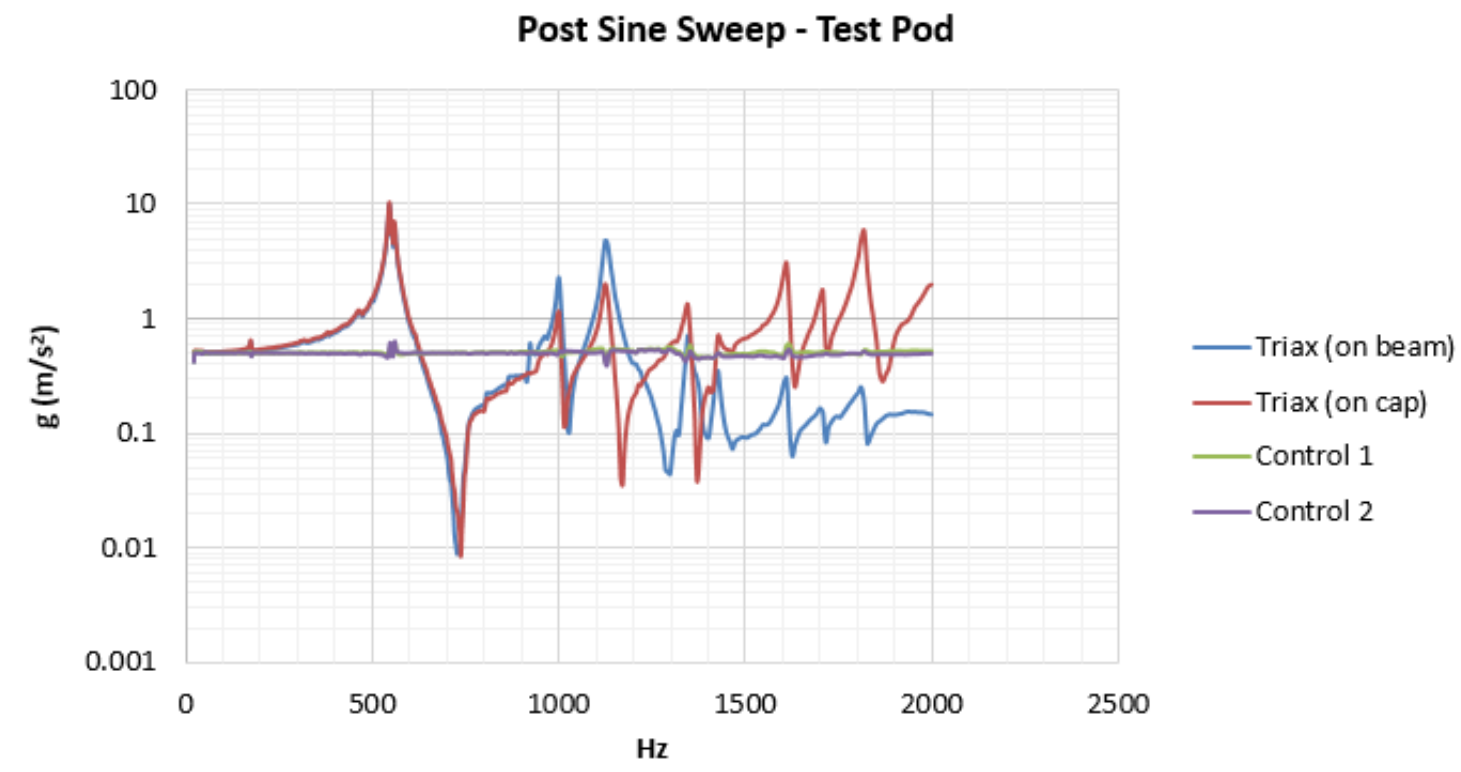

Figure 4.21: Test pod response is similar to that of the pre sine sweep test, indicating no real changes occurred during the random vibration test. 


\subsubsection{Test Pod with Mass Model}

With the mass model integrated in the test pod, testing was repeated in the same manner as was done for the empty test pod assembly, with two sine sweeps and an intermediate random vibration test. Control accelerometers were kept at the same locations of the fixture plate, and triaxial accelerometers were attached to the test pod and mass model (see Figure 4.22).

While the triaxial accelerometers were attached in a different orientation, the axes were aligned in the controller interface to match with the z-axis of the test pod coordinate system, as was done for all previous tests. Also, the ideal case would be to attach the triaxial accelerometer to the main body of the mass model, but it is difficult to securely attach components to the slippery surface of Delrin using adhesives alone. For this reason, it was decided that a steel bracket of the mass model would be the next best location to attach the triaxial accelerometer.

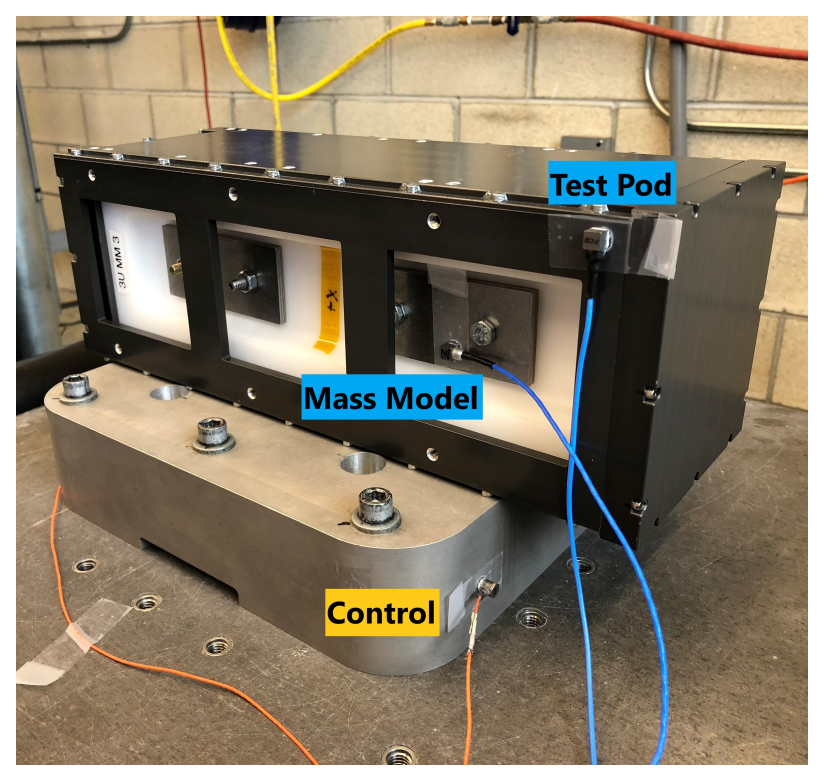

Figure 4.22: Triaxial accelerometers on the test pod and mass model. 


\subsubsection{Pre Sine Sweep}

With an input of $0.25 \mathrm{~g}$ for the first sine sweep test, the test pod and mass model shared a peak response at approximately $450 \mathrm{~Hz}$, with the mass model response being more pronounced at this frequency (see Figure 4.23). Comparing this to the response of the empty test pod in Figure 4.19, it is evident that the first peak response of the test pod as now "shifted" to the left, bringing the first natural frequency of the test pod down from $550 \mathrm{~Hz}$ to $450 \mathrm{~Hz}$. Recalling that $\omega=\sqrt{k / m}$, it can be deduced that the added weight of the mass model to the system is in part a culprit for the smaller natural frequency of the test pod.

The second shared peak occurs around $1190 \mathrm{~Hz}$, with equal magnitudes, but at a less degree than the first peak. The test pod also had a lone response close to $1600 \mathrm{~Hz}$, and while this response resembles the same peak frequency of the empty test pod in Figure 4.19, the existence of a natural frequency at this point is still questionable due to the increased noise levels beyond the $1500 \mathrm{~Hz}$ threshold.

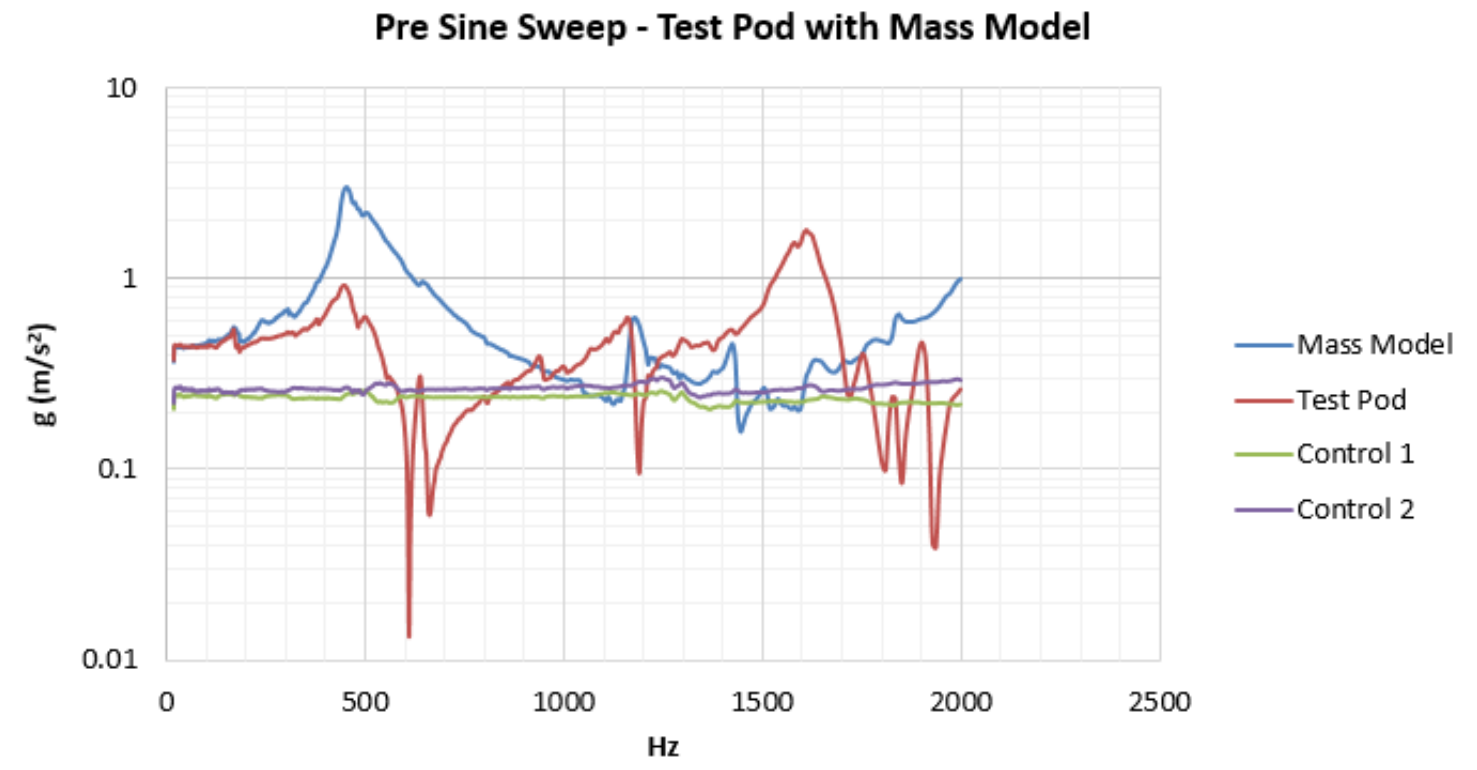

Figure 4.23: The mass model has a more pronounced response than the test pod during the pre sine sweep test. 


\subsubsection{Random Vibration}

The response of the test pod and mass model stayed within the NASA GEVS limits, with a single pronounced peak at approximately $400 \mathrm{~Hz}$ (see Figure 4.24). This peak corresponds to the z-axis of the mass model, which saw the largest excitation during the random vibration test. Note that this behavior corresponds to the previous sine sweep test of Figure 4.23, where the mass model also had a more pronounced response than the test pod at the frequency of $450 \mathrm{~Hz}$. Comparing to the empty test pod response of Figure 4.20, the first peak response of the test pod (z-axis) has gone down from $520 \mathrm{~Hz}$ to approximately $380 \mathrm{~Hz}$ in this test. Again, this shift of the peak frequency indicates that the added weight of the mass model is partly responsible for reducing the natural frequency of the test pod. Also note that although the responses become increasingly chaotic after $1000 \mathrm{~Hz}$, the magnitudes remain within the NASA GEVS limits, as opposed to the response of the empty test pod. So, the inertial forces of the mass model help stabilize the system response.

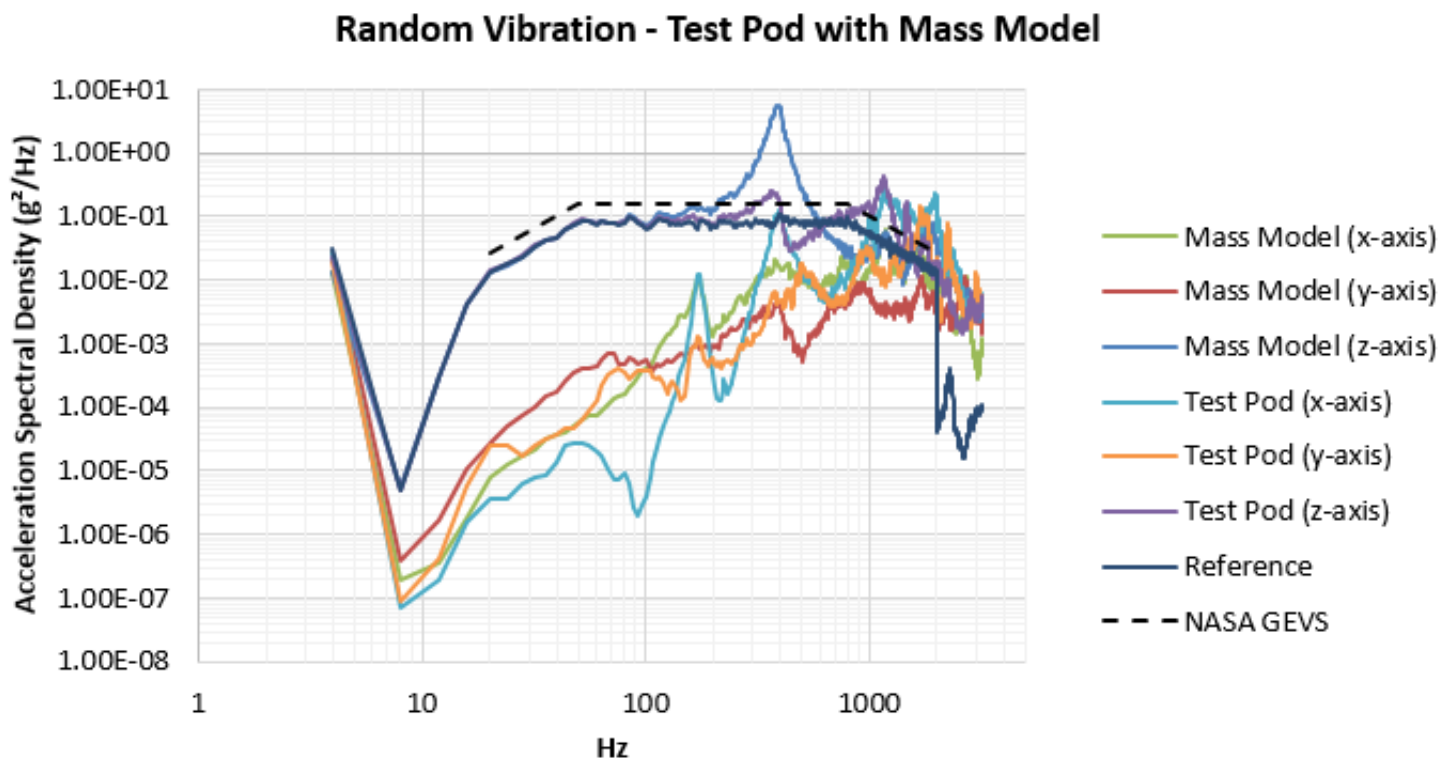

Figure 4.24: Random vibration response of test pod and mass model. 


\subsubsection{Post Sine Sweep}

Compared to the pre sine sweep response of the test pod and mass model, no major changes were observed in the post sine sweep response following the random vibration test (see Figure 4.25). While the magnitude of the first peak response (450 Hz) of the mass model was smaller by about $1 \mathrm{~g}$ when compared to the pre sine sweep data, the peaks of the response stayed relatively the same overall, indicating that the system saw no significant changes during the random vibration test. With these results, it was determined that the test pod and mass model system has natural frequencies at approximately $450 \mathrm{~Hz}$ and $1190 \mathrm{~Hz}$.

Note that the magnitudes of anti-resonance are less pronounced throughout the post sine sweep response. This is possibly due to less destructive interference between the vibration table and the loaded test pod, as a result of more synchronous motion.

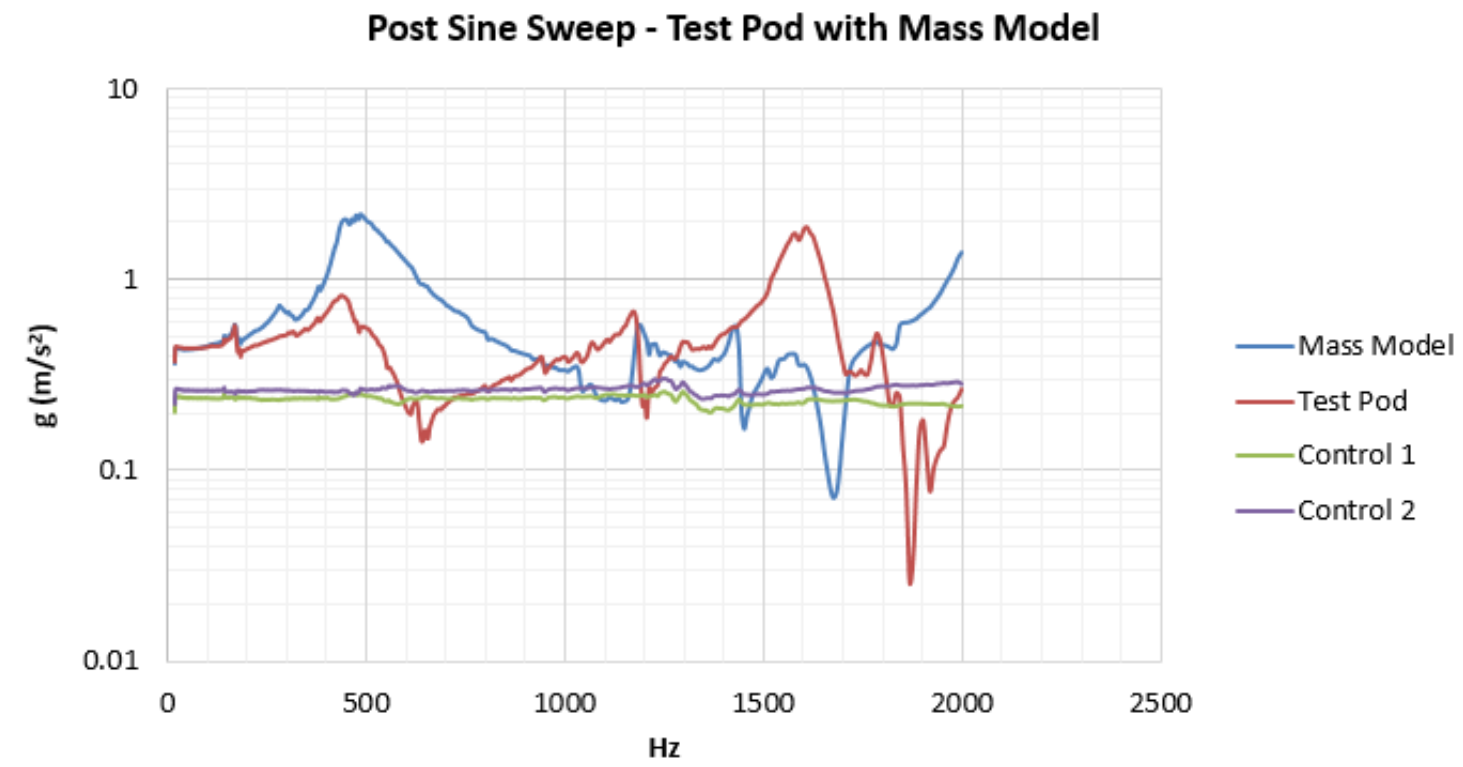

Figure 4.25: Response of the test pod and mass model are similar to that of the pre sine sweep test, indicating no real changes occurred during the random vibration test. 


\subsection{Post-Process of Experimental Data}

With vibration testing completed, all data was collected, organized, and formatted to produce the plots presented in this chapter. This data was used to validate the FEM models to follow, and key test results will be discussed again in Chapter 6 .

In addition, response plots were generated from the data to determine the damping state, or damping ratio $\zeta$, of the empty and loaded test pod setups used in the experiments. However, as all output data was in the frequency domain, it was necessary to first convert this data to a time domain, as this allows the use of techniques discussed in Section 2.1.2 for approximating $\zeta$ of a dynamic system. By applying

$$
u_{n}(t)=e^{-\zeta_{n} \omega_{n} t}\left(\cos \omega_{n} t+\frac{\zeta_{n}}{\sqrt{1-\zeta_{n}^{2}}} \sin \omega_{n} t\right)
$$

with the approximated $\zeta$ value, the time-domain response plots were generated for the empty and loaded test pod using the first natural frequency of their corresponding pre-sine sweep data. The response of the empty test pod decays exponentially, and exhibits an underdamped behavior (see Figure 4.26), as was expected from the estimated $\zeta$ value $(\sim 0.007)$ being less than 1 . Similarly, the loaded test pod also exhibited an underdamped behavior, as expected from the estimated $\zeta$ value for the test pod $(\sim 0.114)$ and mass model $(\sim 0.088)$. Also note that the difference in $\zeta$ value affects the amplitude of the response, as a larger $\zeta$ will reduce the magnitude of the peak response. From the loaded test pod overlay (see Figure 4.27), it is clear that the mass model has a more pronounced response than the test pod for the same natural frequency of the system. Many factors can affect this damping behavior, including the different material properties and physical constraints of the test pod and mass model. Nonetheless, this information gives insight to how CubeSats respond to dynamic stimulation, in comparison to the P-pod or test pod housing. 


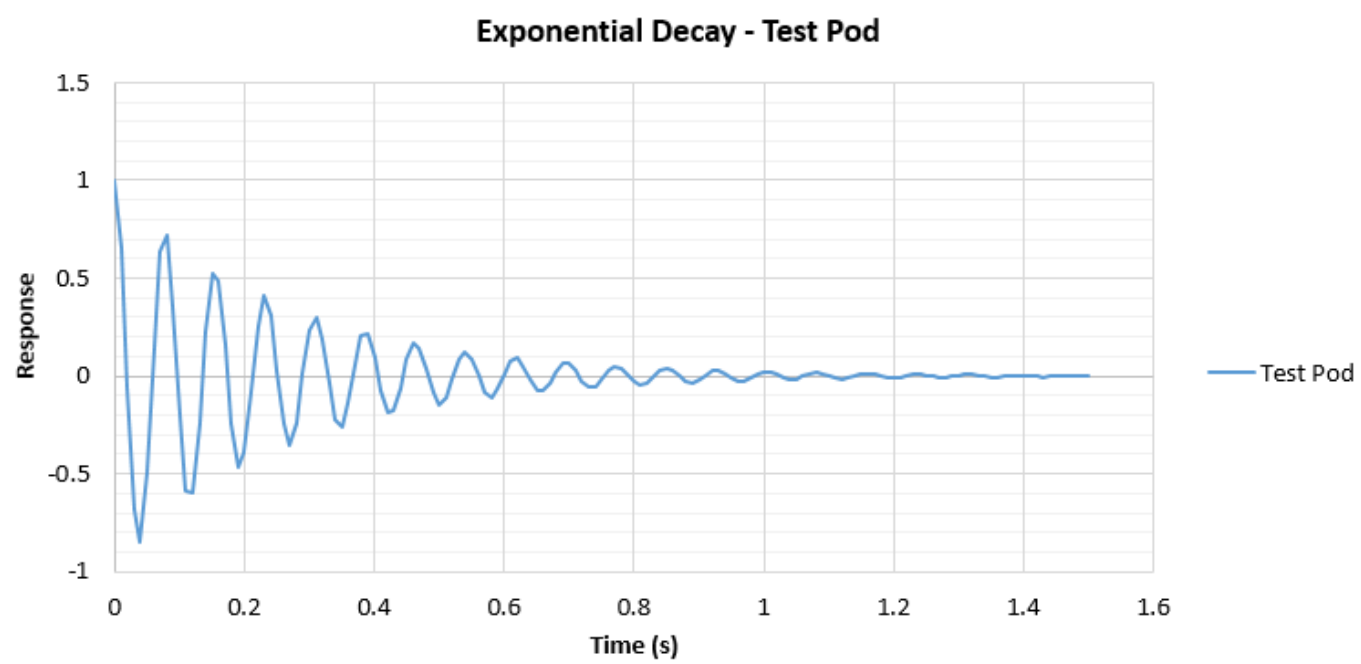

Figure 4.26: Time-domain response of the empty test pod.

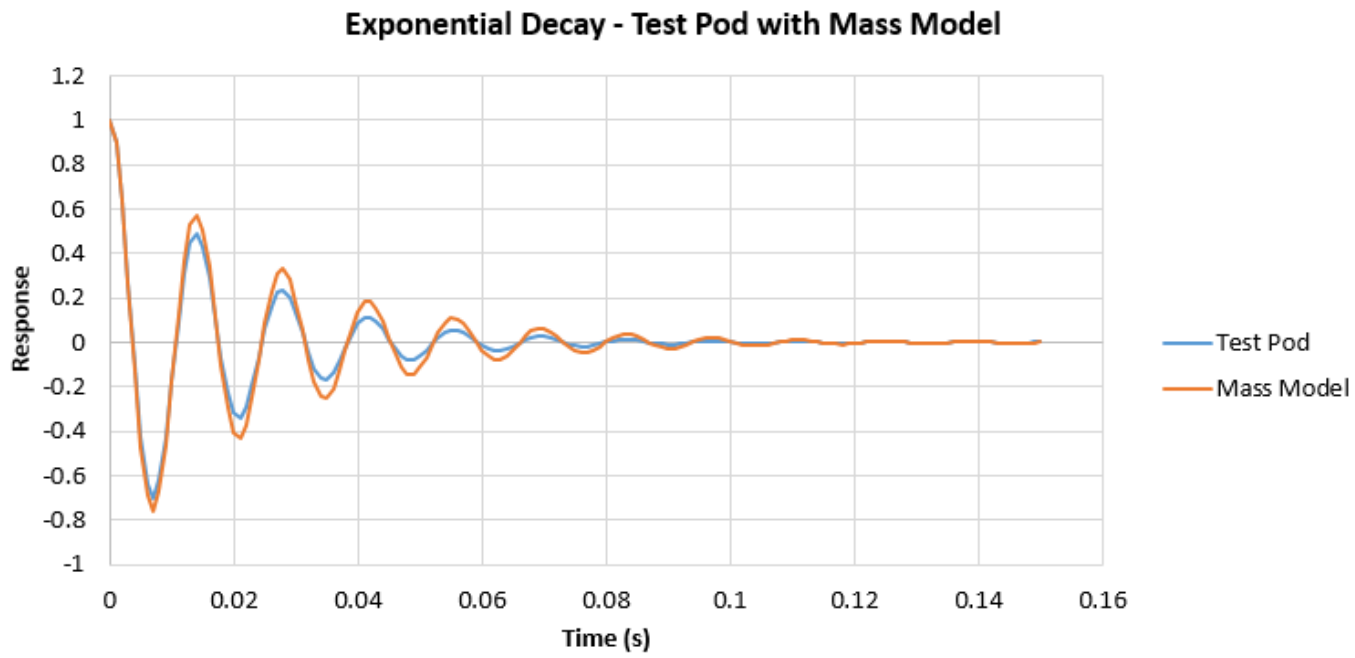

Figure 4.27: Time-domain response of the loaded test pod. 
Chapter 5

FINITE ELEMENT ANALYSIS AND DISCUSSION

\subsection{Early Work}

Prior to building the FEM models, early development first started with using the dynamic analysis tools of the MSC Adams View software. This program is a multibody dynamics simulator, which can be used to study the loads and forces acting on a mechanical system. ${ }^{53}$ Adams View can be integrated with Adams Flex to make a flexible body in order to extract information like deformations, stresses, and mode shapes of the system.

CAD geometry models where imported into Adams, and position constraints where applied to arrange all components in a similar configuration to the actual setup used during testing (see Figure 5.1). Material properties were applied to all components, including the armature CAD bodies which were provided by Unholtz-Dickie Corp. All masses were evaluated in the Adams model to ensure that they reflected the measurements taken during testing, and the information provided by the vendor.

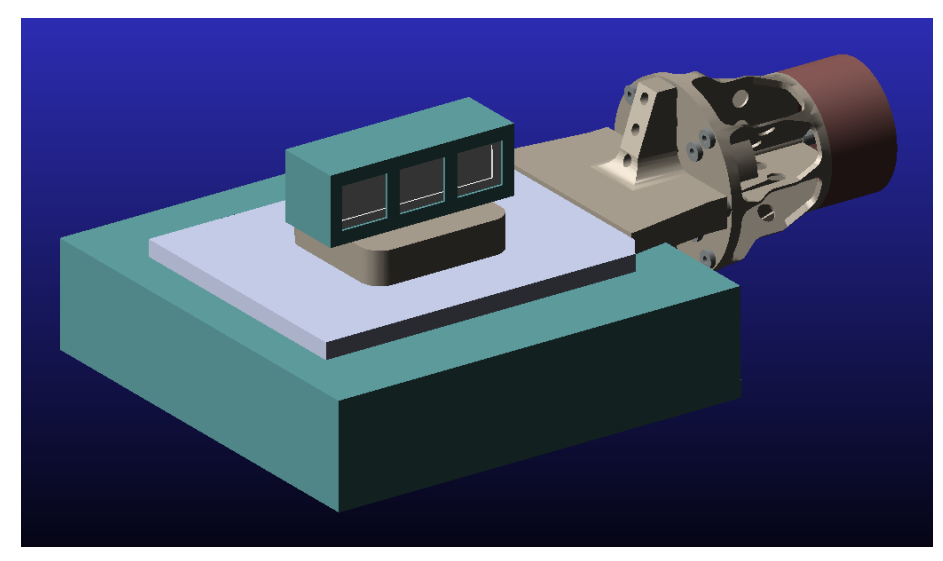

Figure 5.1: Adams View model of the vibration table setup. 
The main challenges became apparent when setting up the interactions and boundary conditions of the model in a way that was representative of the real setup. Planar and translational joints were used to control the motion of the armature assembly, and friction was assumed negligible between the large block base and the slip plate (see Figure 5.2). However, there was no direct way to fix the surface of one body to that of another in the tool options of the program. This in turn made it difficult to set up a fixed constraint that would act only at the test pod and fixture plate interface, without locking both parts as two rigid bodies entirely. This same challenge also applied to the slip plate and fixture plate interface.

Another approach to model the interaction between the test pod and fixture plate was to use flexible connectors known as bushings. These tools can be used to represent the linear force acting over a distance between two separate parts, as shown for the test pod interface (yellow) and slip table interface (red) of Figure 5.2. However, the stiffness and damping properties of each bushing need to be defined for all three axes. This is difficult to obtain through analytical means only, especially when trying to analyze the damping behavior of the system (see Appendix A.2 for more details).

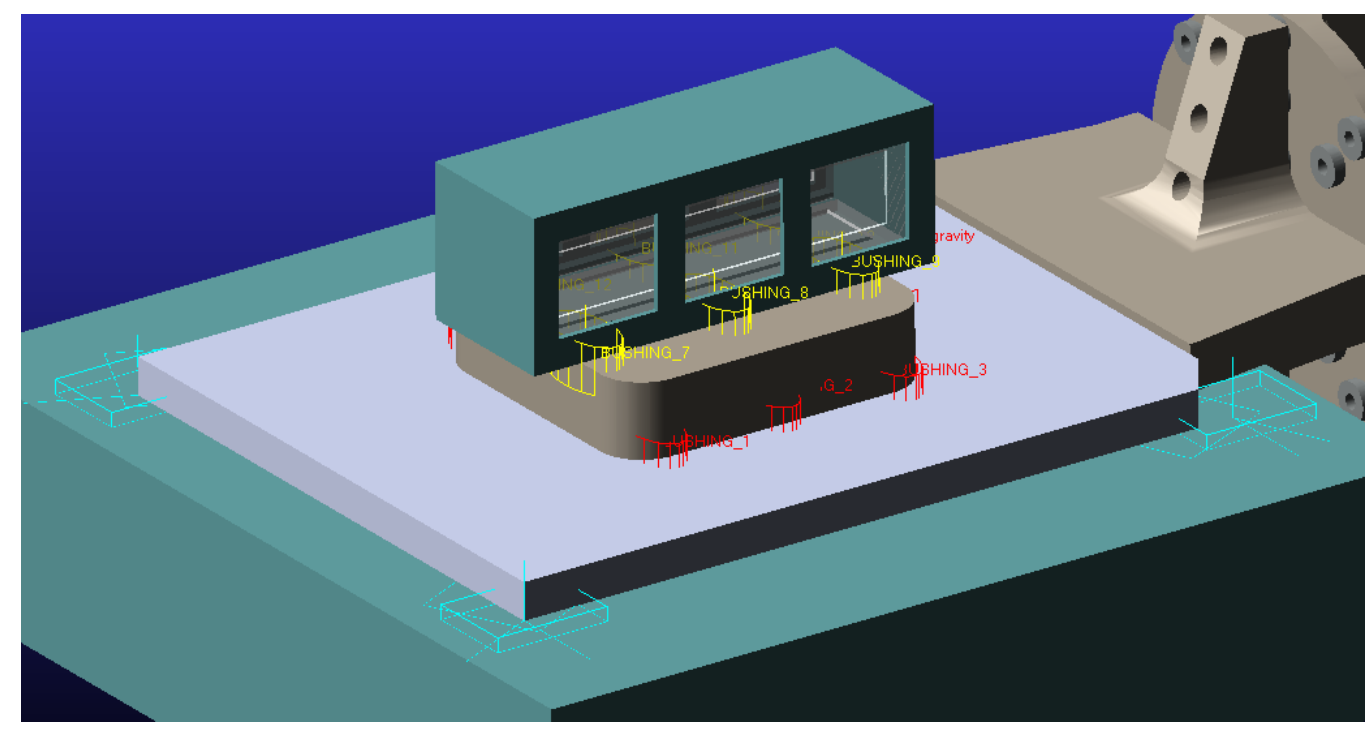

Figure 5.2: Adams model showing planar joints on the large base, and bushing connections at the test pod (yellow) and slip plate (red) interface. 
To gather readings from the model, output channels were set at specified markers on the test pod and mass model (see Figure 5.3). The channels were placed to reflect the location of the accelerometers during the vibration tests of the test pod and mass model assembly.

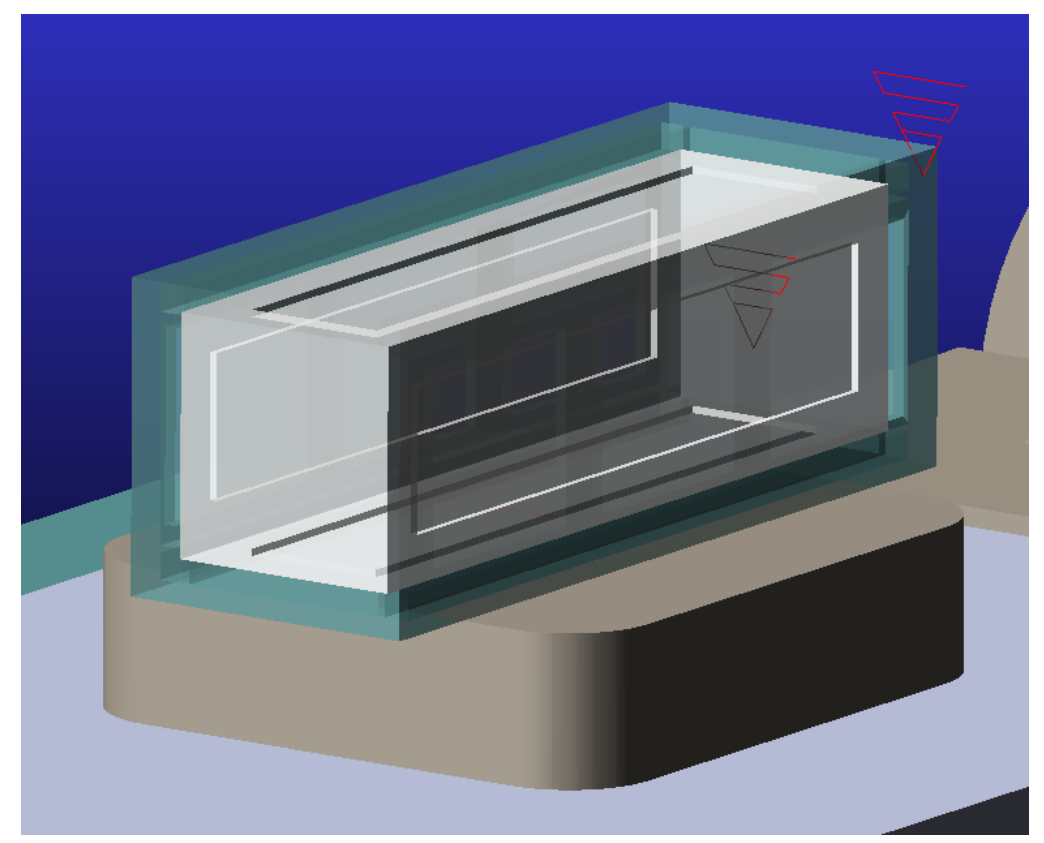

Figure 5.3: Adams model with output channels on the corner of the test pod (transparent) and side panel of the mass model.

For the boundary conditions of the mass model, the intent was to fix one side of the mass model to the internal surface of the test pod cap, but this proved to be difficult for the reason previously mentioned with respect to joining adjacent surfaces. Spring connectors were also used to imitate the spring plunger and floating plate mechanism of the test pod (see Figure 5.4). Similar to the bushing element, stiffness and damping coefficients need to be defined for each spring. Although a high stiffness and damping value could be set, constraining the lateral motion of the mass model to keep it within the bounds of the test pod overcomplicated the Adams assembly. 

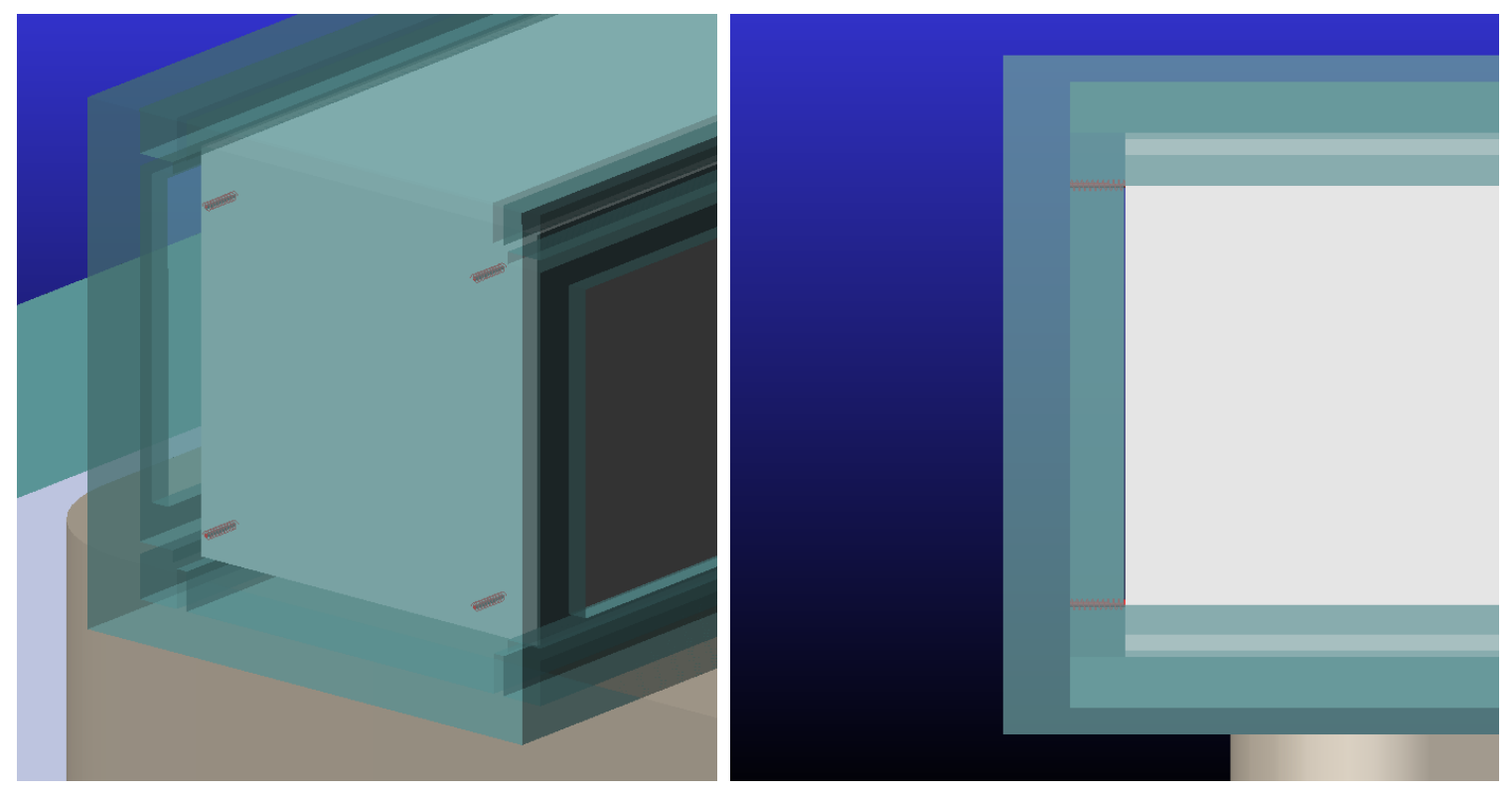

Figure 5.4: Adams model with translational spring-damper connections between the test pod (transparent) and mass model.

As fixed rigid bodies, the test pod and mass model have no degrees of freedom available to solve for deformation and subsequent modes of vibration. So as a final attempt, the test pod was converted to a flexible body (see Figure 5.5), where Adams uses a built-in MSC Nastran subroutine to dicretize the body. It should be noted that although this coding differs to that used by most FEA programs, MNF files generated by FEA programs can also be imported into Adams for analysis.

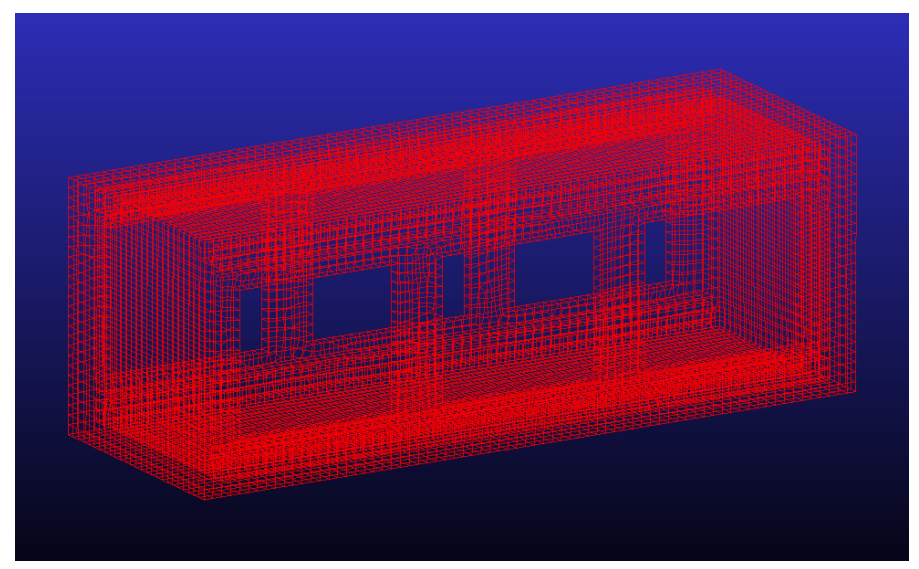

Figure 5.5: Adams model of the test pod as a flexible body. 
To simplify the model, the vibration table armature assembly was omitted from the analysis, using only the test pod and fixture plate (see Figure 5.6).

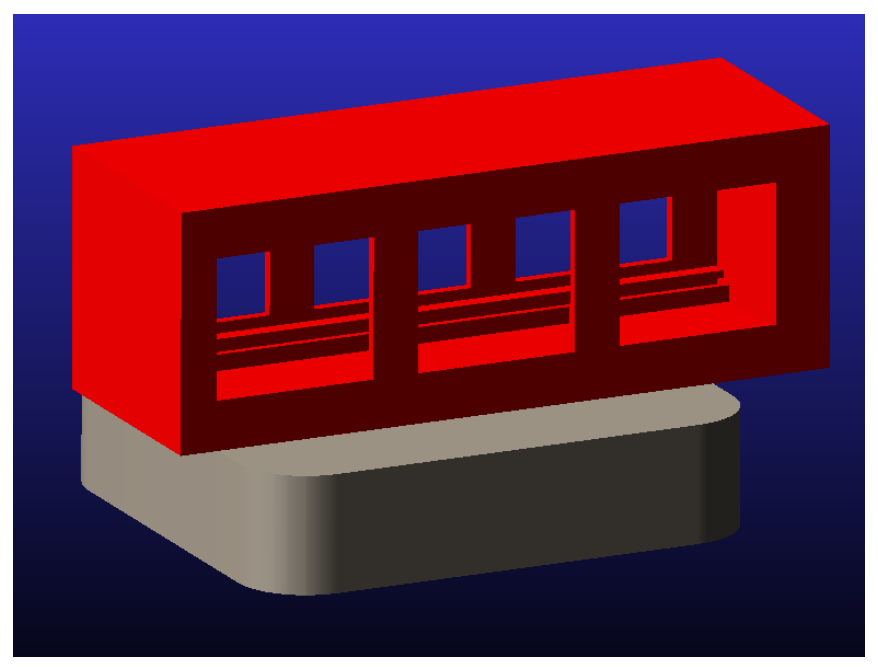

Figure 5.6: Adams model of the test pod and fixture plate.

Six fixed joints were created to represent the approximate locations of the screws used in the testing assembly (see Figure 5.7). Although these type of joints do not constrain surfaces, they can constrain the nodes of adjacent bodies at the selected datum coordinate system. A translational joint (not shown) was also used to restrict all degrees of freedom of the fixture, except along the z-axis.

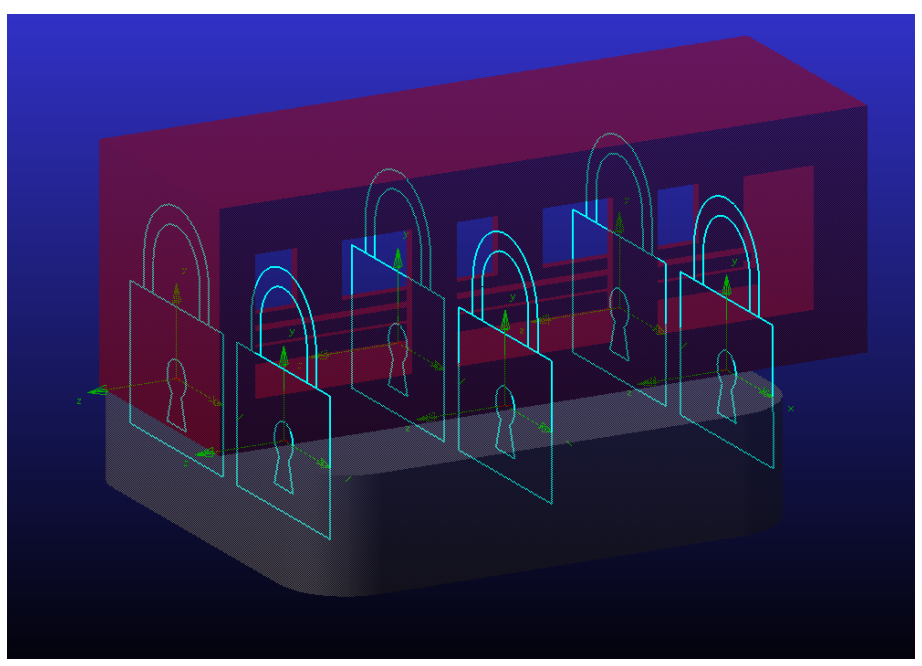

Figure 5.7: Fixed joints are used to constrain the test pod to the fixture. 
Similar to the accelerometer locations used during the vibration tests, output channels were placed at the beam and cap of the test pod (see Figure 5.8). A reference output channel was also added at the center of the fixture in order to confirm a $0.5 \mathrm{~g}$ input along the z-axis of the assembly (see Figure 5.9).

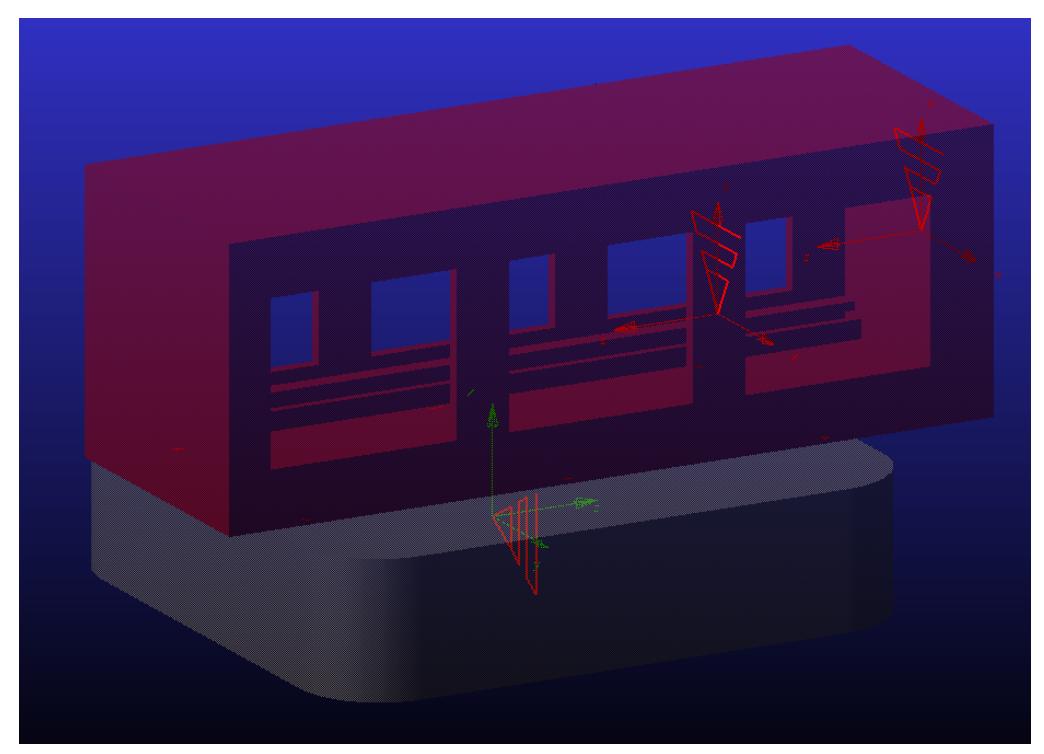

Figure 5.8: Output channels at the cap and beam of the test pod. A reference output is also located at the center of the fixture.

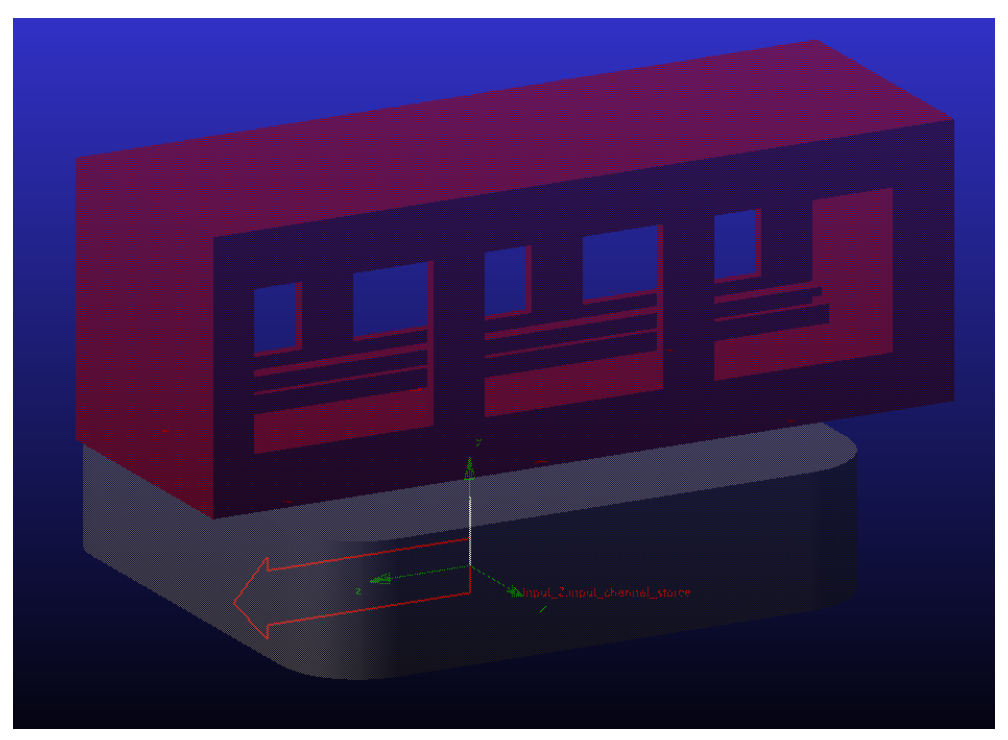

Figure 5.9: $0.5 \mathrm{~g}$ input at the bottom surface of the fixture plate. 
Running a sine sweep analysis in Adams from 0-2000 Hz with the $0.5 \mathrm{~g}$ input, the assembly has a peak response at approximately $600 \mathrm{~Hz}$ (see Figure 5.10). This frequency is in close proximity to the first peak response observed in the experimental data of the test pod $(550 \mathrm{~Hz})$. So although a crude assembly with simplified constraints was used for the analysis, the Adams model predicts the first peak response with reasonable accuracy.

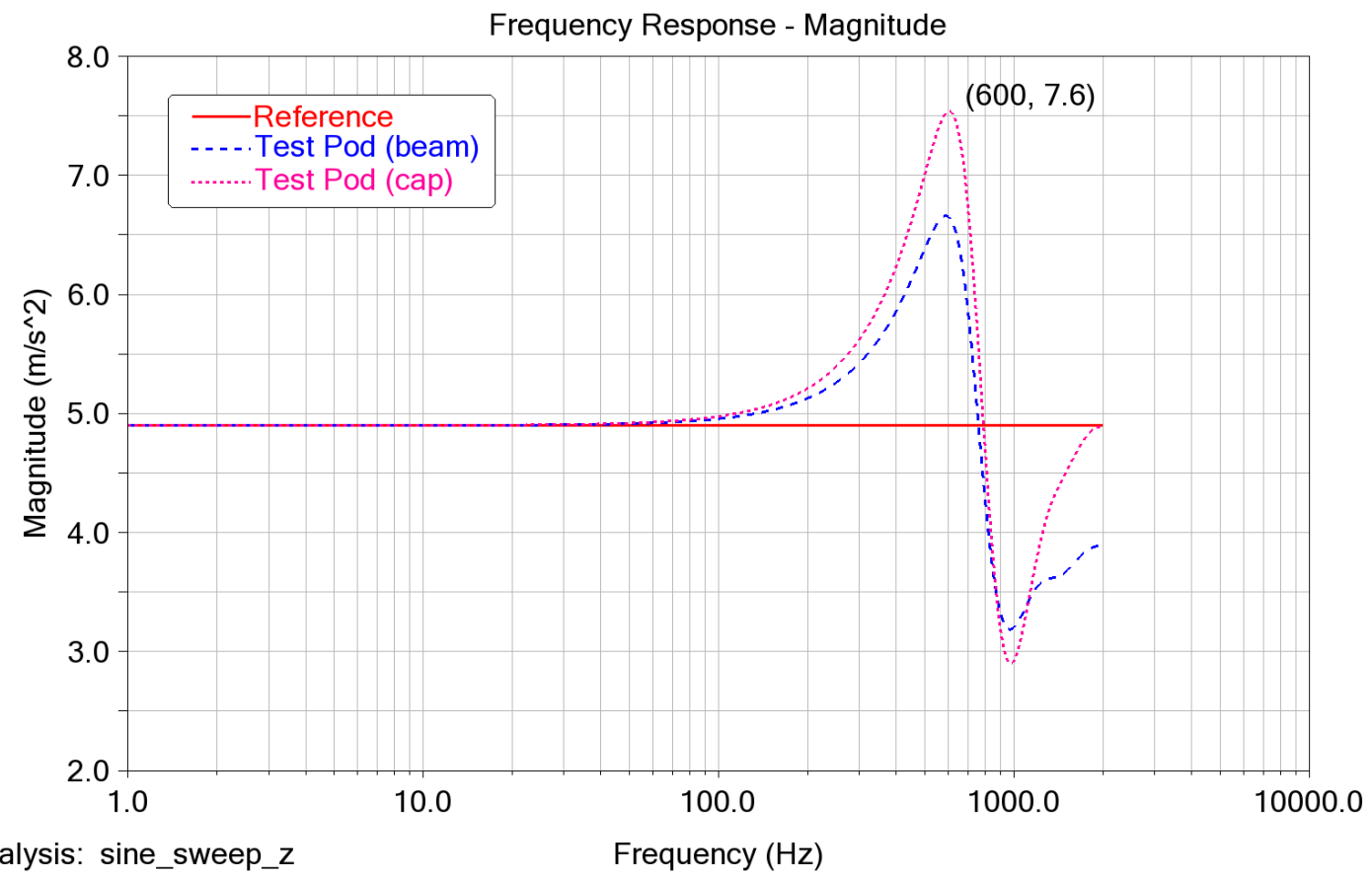

Figure 5.10: Adams model of the test pod assembly has a peak response near $600 \mathrm{~Hz}$.

While it proved challenging to build upon these models to fully realize the scope of this work, the successful modeling of the test pod assembly in Adams provided some validation to the CAD models, constraints, and boundary conditions being used to represent the real system. With positive results observed in Adams, work continued to build upon this model using more robust finite element software. 


\section{$5.2 \quad$ Finite Element Analysis}

Abaqus is a commercial software program used for finite element analysis (FEA) of many different structures and systems. Various packages are available including Abaqus Standard, Explicit, and CAE (Complete Abaqus Environment). Abaqus Standard uses traditional implicit integration to solve general-purpose FEA problems, Abaqus Explicit applies more involved explicit integration to solve systems with highly non-linear behavior, and Abaqus CAE provides a visualization interface as part of the post-processing for an FEA model. ${ }^{6}$ The Abaqus subroutine applies the finite element method to discretize a body into small elements that can be individually analyzed to determine the behavior of the body as a whole. In addition, results like stress and displacements can be displayed in the body for a visual understanding of the system behavior (see Figure 5.11).

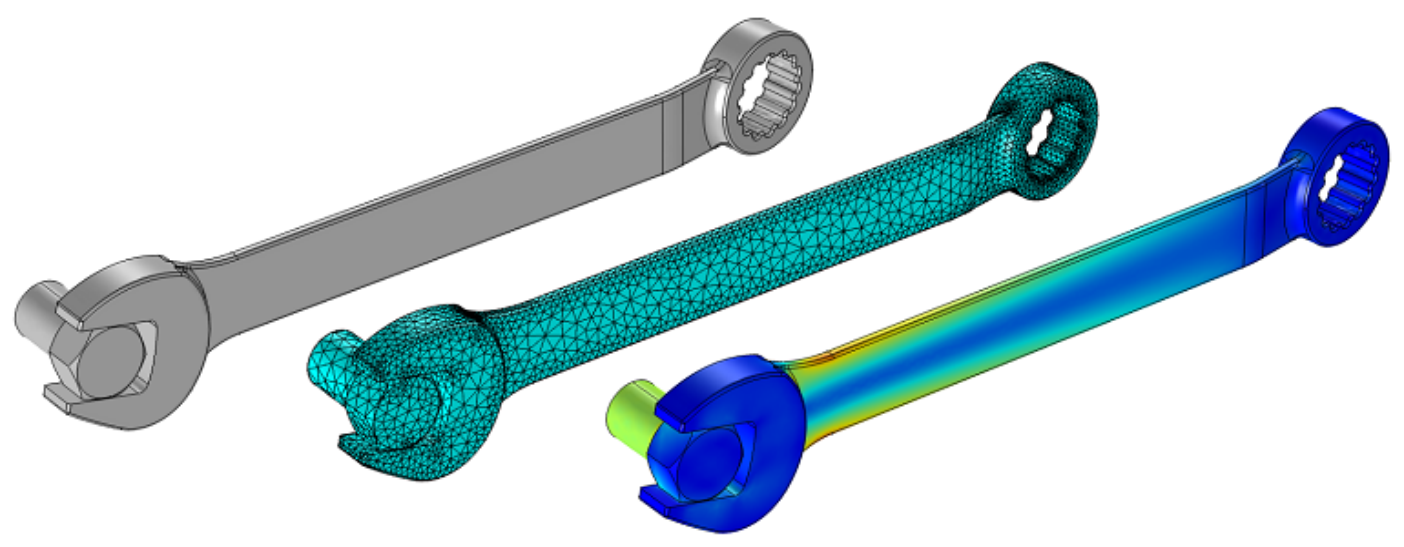

Figure 5.11: FEA model of a wrench and bolt with stress distributions. ${ }^{57}$ 
To determine the best approach for building the FEA models in Abaqus, special factors were considered about the test setup, and CubeSats in general. First, due to the relatively small displacements that are generally observed in the dynamic testing of CubeSats, it can be assumed that the stiffness of the test pod assembly does not change over the time span of a vibration test, not to mention the more rigid fixture plate. Recalling from FEM that $\{F\}=[K]\{d\}$, the stiffness matrix $[K]$ of the system can represent anywhere from a few to several million linear algebraic expressions, depending on the size of the FEA model. So if the stiffness is non-changing, the matrix needs to be solved only once throughout the analysis, as opposed to the much more time-consuming iterative process of a stiffness matrix that changes with time (non-linear behavior). ${ }^{76}$

Second, the test pod and fixture plate are composed of aluminum and magnesium, respectively, which are both isotropic materials that exhibit linear behavior within their elastic range. Since the deformations of CubeSats are assumed to be small during testing, then it can be deduced that the geometry shape and material properties will not change significantly. Note that although the mass model is composed of Delrin, which is a thermoplastic that generally exhibits non-linear behavior, it is assumed in the FEM models that the mass model is constrained and fixed to the test pod as a way to simplify the analysis. So the mass model is assumed to follow, but not necessarily mimic, the behavior of the test pod.

Based on these main points, a linear analysis using Abaqus Standard was chosen to the develop the FEA models for the test pod, mass model, fixture plate, CPX, and their corresponding assemblies. 


\subsubsection{Test Pod}

To begin setting up the FEA models in Abaqus, the test pod CAD geometry was imported from SolidWorks. In Abaqus, partitions are used to break up a complex body into smaller sections in order to simplify the analysis. This also provides added control of the dicretization process, where different properties can be assigned to different regions of a body. For the test pod, the partitions were created in way that generated a reasonably uniform pattern of discretization in the body geometry (see Figure 5.12).
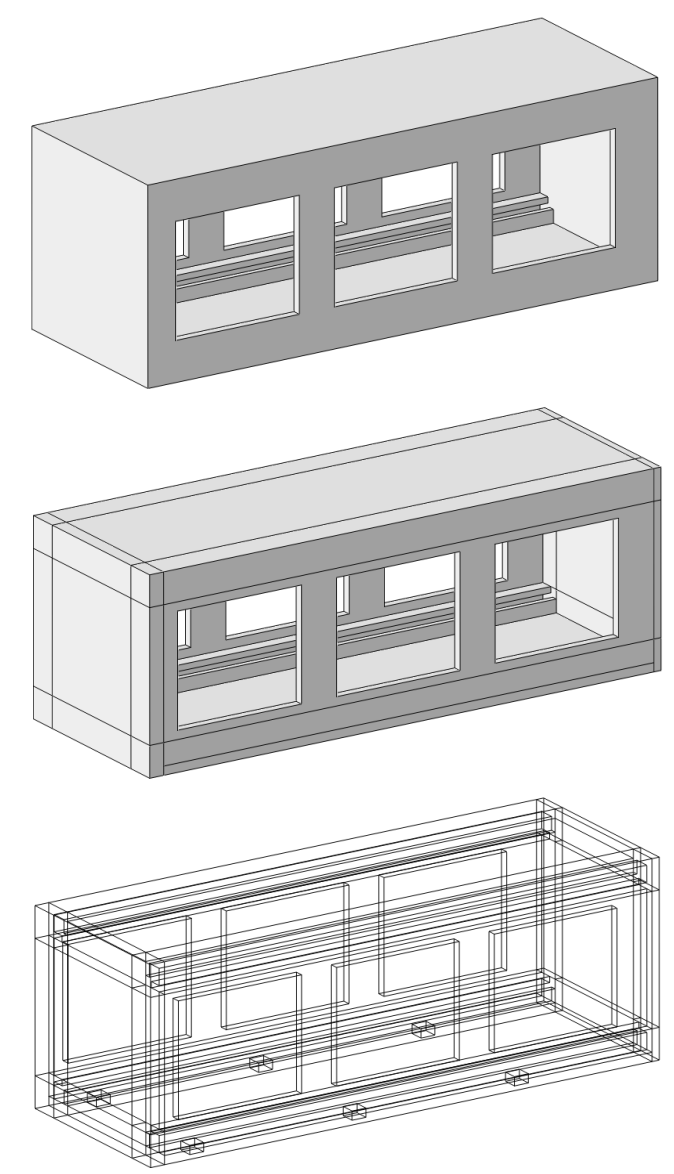

Figure 5.12: Test pod CAD (top), FEA partitions (middle), and wireframe view (bottom). 
Note from the wireframe view of Figure 5.12 that six small partitions are visible at the base of the test pod. These were created in the FEA model to represent the locations of the six screws used to assemble the test pod to the fixture plate (see Figure 5.13). During vibration, loads around the screw holes of the thin test pod behave differently, as opposed to the remaining surface of the test pod base. As these screw areas are the attachment points of the assembly, the FEA partitions were created to reflect these boundary conditions.

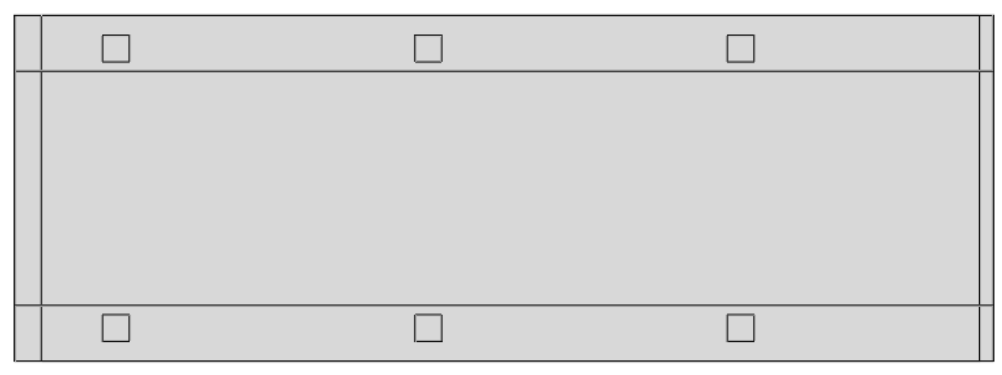

Figure 5.13: Small square partitions on the bottom panel of the test pod FEA model.

\begin{abstract}
Although one partition layout was used for the test pod, mass model, and fixture plate, there are many ways of partitioning a body and is a skill that is learned with experience over time while working with various FEA models. The partitions used for all FEA models here were adequate enough to carry out the analysis with satisfactory results.
\end{abstract}




\subsubsection{Mass Model}

Like the test pod, the mass model CAD geometry was imported into Abaqus for development of the FEA model. The mass model CAD was designed as a simple and symmetric body, and as such, the amount of partitions required for the FEA model was minimal (see Figure 5.14). The square panels at both ends of the mass model were kept free of partitions as these faces sit flush with the test pod cap and floating plate, with the assumption that the load distribution is uniform throughout the surface of the two panels.

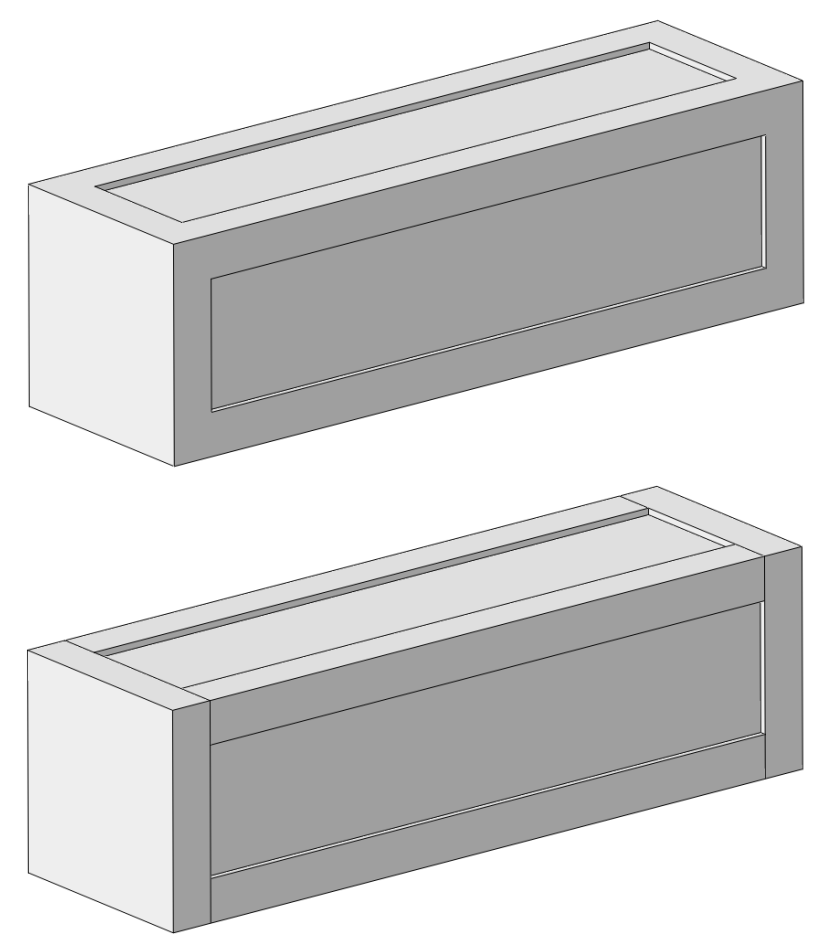

Figure 5.14: Mass model CAD (top) and FEA partitions (bottom). 


\subsubsection{Fixture Plate}

The fixture plate CAD model required few partitions due to the simple and symmetric shape of the body (see Figure 5.15). Partitions were added to the rounded corners of the FEA model to localize any irregular-shaped elements that would be needed to form the contours of the rounded corners (recall FEM elements have straight edges). The fixture plate was assumed to be rigid enough to not need any additional partitions for particular boundary conditions, as the thick and stiff body of the fixture plate sees minute deformations during a vibrations test, even with the loaded test pod attached.
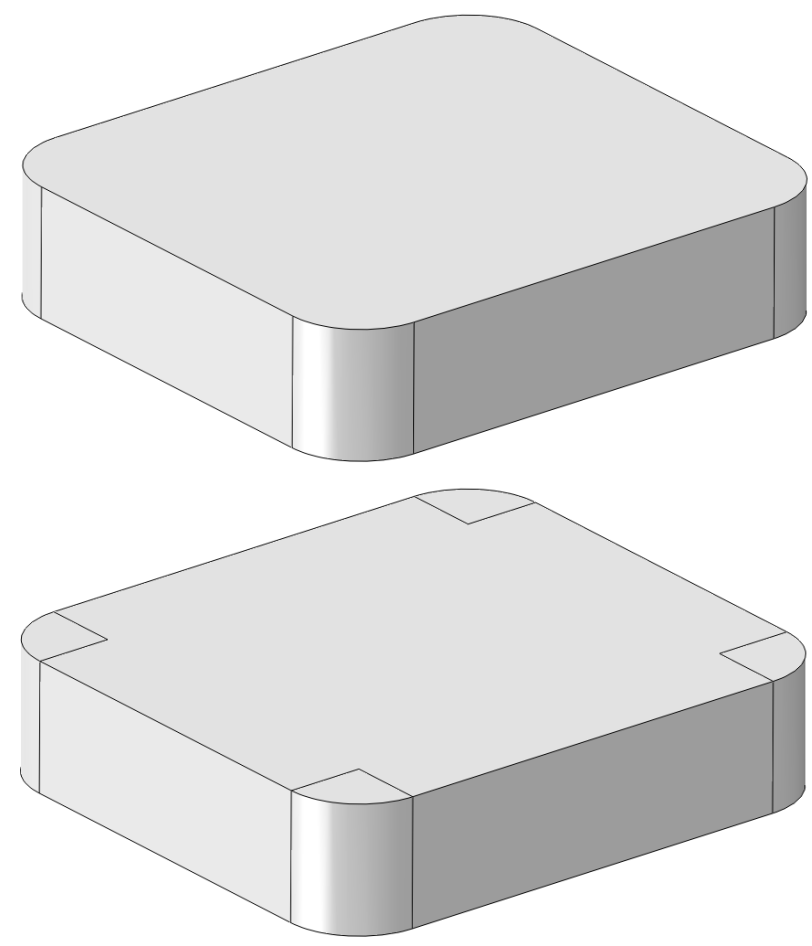

Figure 5.15: Fixture plate CAD (top) and FEA partitions (bottom). 


\subsubsection{Assembly}

The FEA models of the test pod, mass model, and fixture plate (see Figure 5.16) were assembled using constraints similar to those used in the SolidWorks CAD assembly (revert to Section 3.2.4). Note that position constraints are not enough to define an FEA assembly, as relationships between elements of different components must also be established in the assembly model.
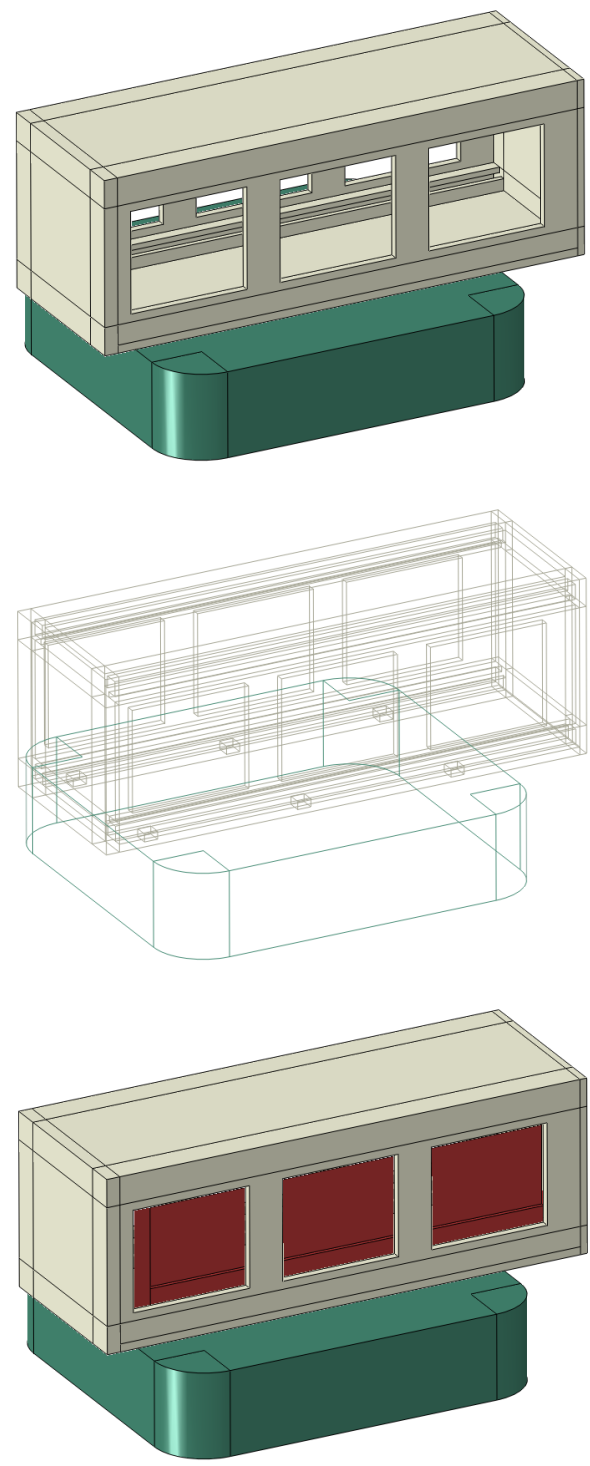

Figure 5.16: FEA model of the empty test pod assembly (top), wireframe view (middle), and loaded test pod assembly (bottom). 


\subsubsection{Interactions and Constraints}

Interactions and constraints are used to establish relationships between the elements and nodes of different bodies in an FEA assembly. There are numerous types of interactions and constraints available in Abaqus, and are applied based on the nature of the model and the type of boundary conditions that need to be established. Such relationships that can be defined between two or more bodies include contact pressure, friction, coupling, heat transfer, and fluid films. For the case of the test pod assembly here, only contact and friction conditions were considered in the FEA assembly.

A surface-to-surface contact interaction was used to define the relationship between the adjacent surfaces (elements) of the test pod and mass model (see Figure 5.17). A contact interaction property must also be defined with the interaction to define the relative tangential and normal motions of the surfaces in contact. For this, recall that friction exhibits non-linear behavior (see Appendix A.4 for more details) and complicates the analysis due to the numerous equilibrium points of oscillation that it introduces to a vibration system. Since the mass model was set to be fixed in the test pod body, it was assumed that friction would be negligible in the FEA model. For these reasons, a frictionless property was chosen for the surface-to-surface interaction.

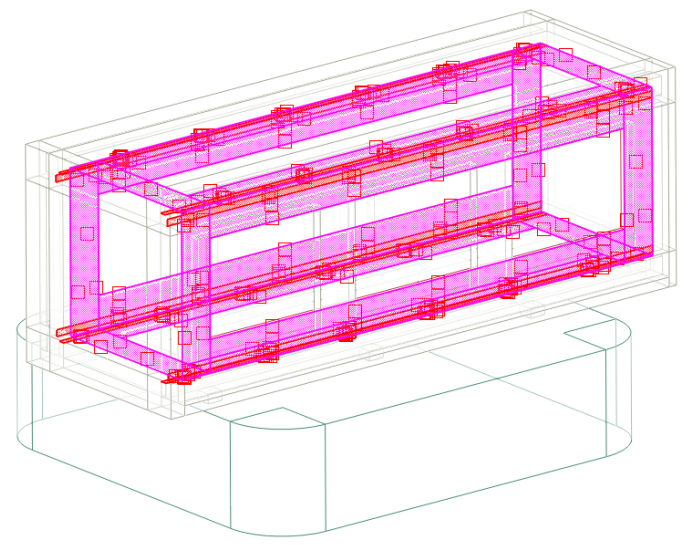

Figure 5.17: Interaction between the mass model (purple) and test pod (red) surfaces of the FEA assembly. 
Tie constraints are surface-based, where the nodes of one surface become "tied" to the nodes of a nearby surface. Such constraints can be used to model welds and bolted joints, where translational and rotational motion can be transferred from one body to another. As such, a tie constraint was used to fix the small screw partitions of the test pod to the top face of the fixture plate (see Figure 5.18). In addition, a second tie constraint was used fix the mass model panel to the internal surface of the test pod cap (see Figure 5.19).

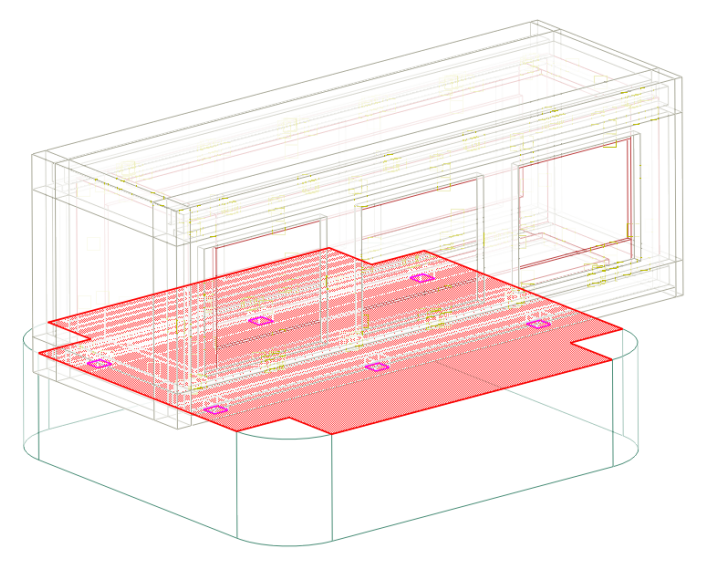

Figure 5.18: Tie constraint between the small partitions of the test pod (purple) and the top surface of fixture plate (red).

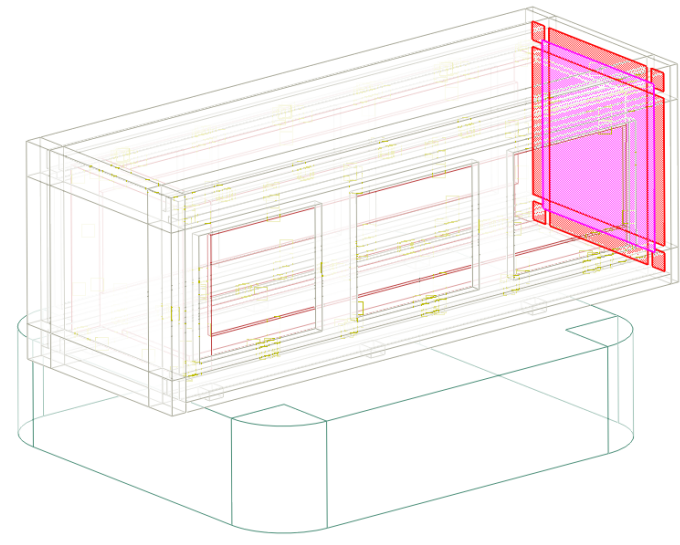

Figure 5.19: Tie constraint between the face of the mass model (purple) and the internal surface of the test pod cap (red). 


\subsubsection{Meshing}

The discretization process of a body in FEA is referred to as meshing, and like partitioning, is a skill that can be improved thru experience while working with various FEA systems and models. Several properties are used to define the mesh of a body, where the selected element shape, type, and size are crucial in developing an adequate mesh that can achieve accurate results from the analysis. The mesh influences the behavior of the elements, and consequently, determines the behavior of the body or system as a whole.

\subsubsection{Element Shape}

Recall from finite element theory (revert to Section 2.2) that various element shapes are available for the analysis of an FEM model, each suited to a particular type of loading and dimension of the FEM model. Since the test pod, mass model, and fixture plate are considered solid bodies, 3D element shapes were chosen to build their corresponding FEA models (see Figure 5.20).

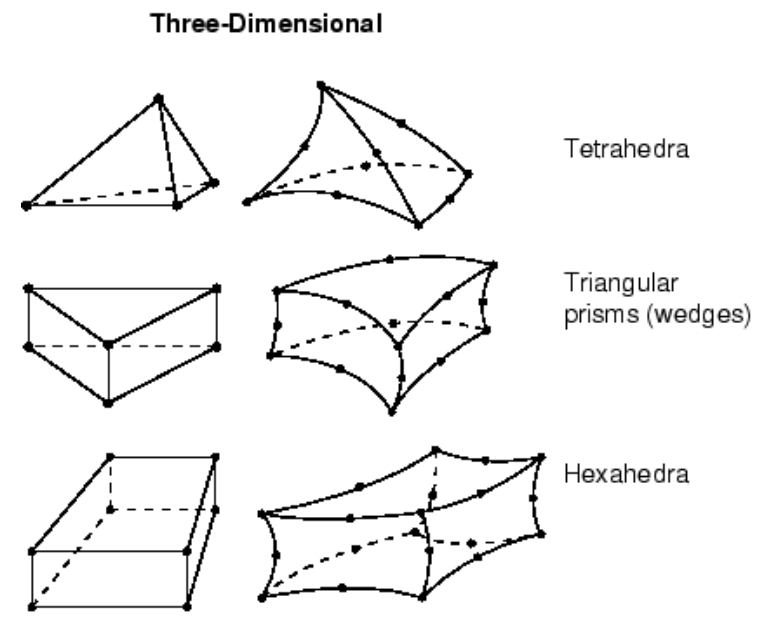

Figure 5.20: Various 3D element shapes that can be used in FEA models. ${ }^{11}$ 
Quadratic (10-noded) tetrahedral elements are quite versatile, and can be applied to many types of FEA models, including those with complex geometries. They can also model bending motion to an accurate degree, ${ }^{20}$ which makes them one of the favored elements in FEA. While this element has its benefits, more of these elements are required to model a body due to its shape, which in turn requires more computation time to solve the model. In addition, since deformations from bending are not significant in the test pod assembly, tetrahedral elements were not chosen for the FEA models.

Hexahedral ("brick") elements are another example of 3D elements (see Figure 5.21) that are commonly used for FEA models of solid bodies. They are robust elements, and can be more accurate than tetrahedral elements in many cases due to their mathematical formulation. ${ }^{20}$ However, with the exception of quadratic hexahedrals, brick elements cannot model bending and may suffer from shear locking, ${ }^{85}$ which falsely makes the FEA model stiffer than what it should be (see Appendix B.3.3 for more details). Fortunately, since bending is not significant in the test pod assembly during vibration, it does not need to be modeled in the FEA assembly. So, with no significant bending and the relatively simple geometries of the four structures, linear hexahedral elements were chosen to build the FEA models for the test pod, mass model, CPX, and fixture plate.

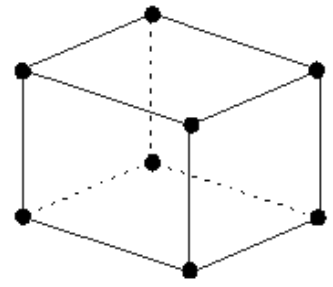

(a) Linear element (8-node brick, C3D8)

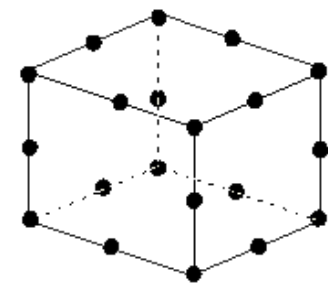

(b) Quadratic element (20-node brick, C3D20)

Figure 5.21: Linear vs Quadratic hexahedral elements. ${ }^{36}$ 


\subsubsection{Element Type}

There are several options that accompany FEM elements, and are based on the element shape and application of the FEA model. For brick elements, two family types are applicable for the dynamic analysis of the test pod assembly. The first family is referred to as solid (continuum) elements, which are standard volume elements used in Abaqus (see Figure 5.22). They are used for general FEA applications with single homogeneous materials, and where distortions in the model are assumed to be minimal. ${ }^{7}$ However, these type of brick elements are still sensitive to changes in their shape, and require a significant amount of computations due to the integration subroutine used to solve each element. This can be a time-costly option, especially for large FEA models with many elements.
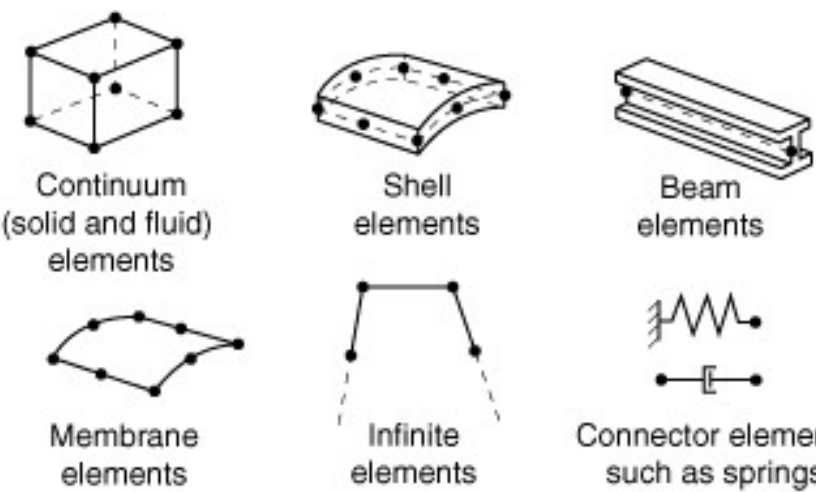

elements
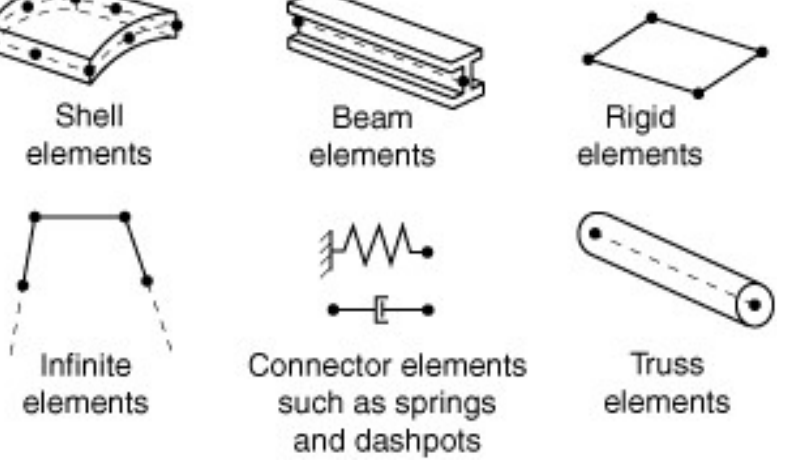

Figure 5.22: Various element types that can be used in FEA models. ${ }^{8}$

Continuum (solid) shell elements are another family type available for brick elements in Abaqus. Shell elements, as shown in Figure 5.22, are excellent for modeling bending, but are restricted to 2D planes. Brick elements can be used for 3D models, but suffer from shear locking while under bending. On the other hand, continuum shell elements combine the benefits of conventional shell elements, while maintaining the full 
response of 3D solid elements ${ }^{29}$ (see Figure 5.23). Continuum shell elements discretize a solid 3D body, but assign a thickness to the "shell" of the body using the nodal geometry. This approach is more computationally efficient than solid (continuum) elements, and is ideal for uniform bodies and thin-walled structures ${ }^{29}$ like the test pod. For these reasons, the continuum shell element type was chosen for the linear brick elements of the test pod, mass model, CPX, and fixture plate FEA models.

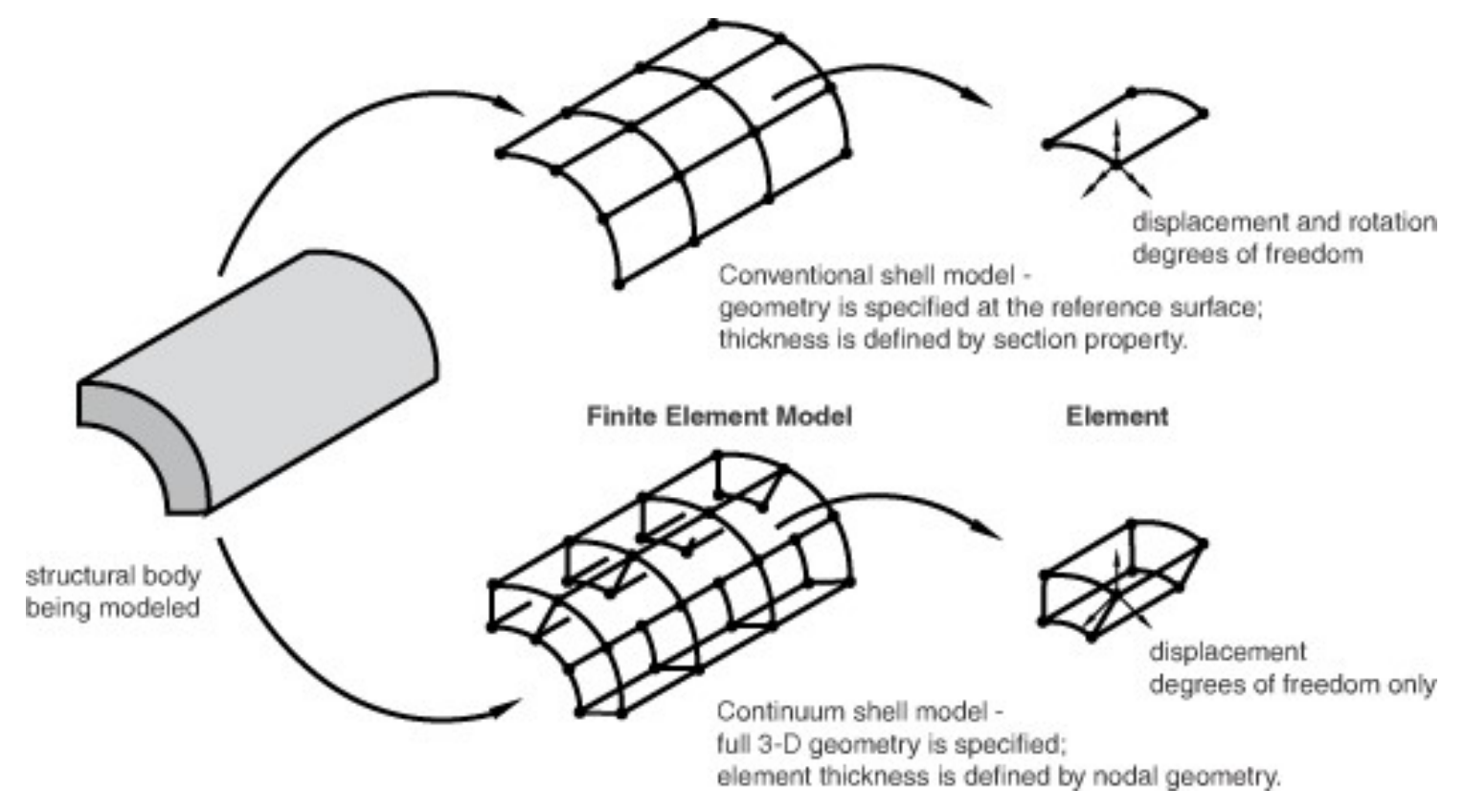

Figure 5.23: Conventional vs Continuum (solid) shell elements available in Abaqus. ${ }^{9}$

\subsubsection{Element Size}

Finally, the size of the elements, or seed size, determines the level of accuracy that can be achieved from the FEA model. To determine a suitable element size, a mesh convergence study is performed on the model to determine where a node parameter (e.g. stress) begins to converge regardless of the number of elements used in the model. Since the FEA models were built for a dynamic analysis, convergence studies were performed using the first set of natural frequencies (see Appendix D for more 
details). Repeating the study for all three components, it was determined that an element size of $4 \mathrm{~mm}, 5 \mathrm{~mm}$, and $7 \mathrm{~mm}$ be used for the test pod, mass model, and fixture plate, respectively.

With the element shape, type, and size now defined, the FEA models of the test pod, mass model, and fixture plate were successfully meshed (see Figure 5.24) and assembled accordingly.
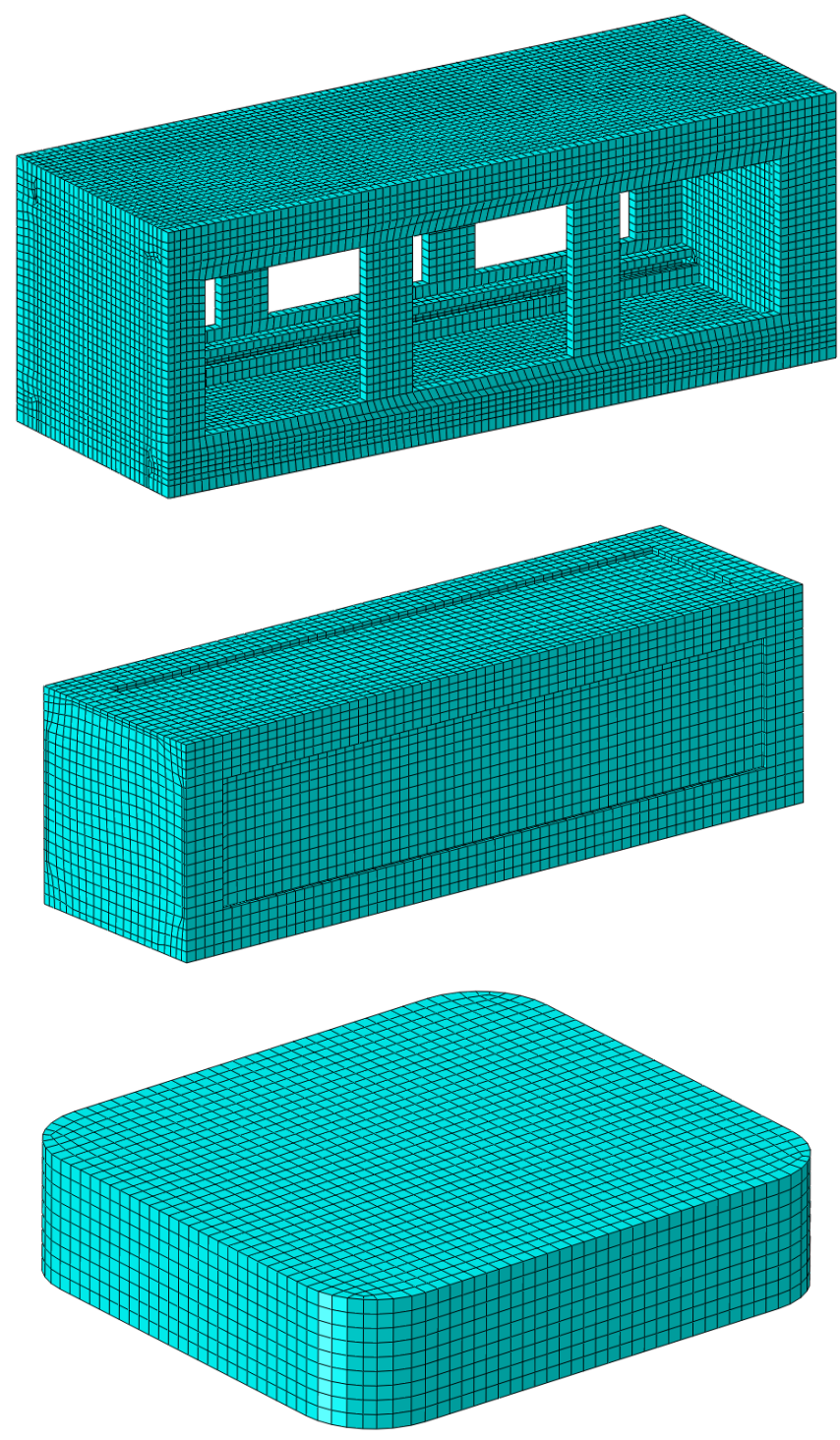

Figure 5.24: Meshed FEA models of the test pod (top), mass model (middle), and fixture plate (bottom). 


\subsubsection{Additional FEA Model Parameters}

\subsubsection{Properties}

Material properties must be assigned to the meshed bodies of the FEA models, and recalling that the test pod, mass model, and fixture plate are composed of aluminum, Delrin, and magnesium, respectively, properties were assigned accordingly using the values of Table 5.1.

Table 5.1: List of material properties used for FEA models.

\begin{tabular}{|c|c|c|c|}
\hline Material & $\begin{array}{c}\text { Mass Density } \\
\mathbf{k g} / \mathbf{m}^{\mathbf{3}}\left(\mathbf{l b} / \mathbf{~ i n}^{\mathbf{3}}\right)\end{array}$ & $\begin{array}{c}\text { Young's Modulus } \\
\mathbf{G P a}\left(\mathbf{1 0}^{\mathbf{6}} \mathbf{p s i}\right)\end{array}$ & Poisson's Ratio \\
\hline Aluminum & $2770(0.10)$ & $71(10.0)$ & 0.33 \\
\hline Magnesium & $1700(0.06)$ & $45(6.5)$ & 0.35 \\
\hline Delrin & $1410(0.05)$ & $2.9(0.4)$ & 0.30 \\
\hline
\end{tabular}

\subsubsection{Loads}

The process of extracting the natural frequencies of an FEA model ignores any loading applied to the system, and instead relies on the boundary conditions (and any initial conditions) set in the FEA model. As such, no loads were applied during the natural frequency and random vibration analysis of the test pod assemblies. However, the damping behavior of the FEA test pod assembly was also analyzed, where an instantaneous $1 \mathrm{~N}$ concentrated force was applied in order to observe the system response of the FEA assembly. 


\subsubsection{Steps}

The frequency step in Abaqus extracts the eigenvalues of the FEA model to calculate the natural frequencies and their corresponding mode shapes. This step was used to determine the natural frequencies of the empty test pod, test pod with mass model, and test pod with CPX assemblies. Their mode shapes were also inspected to identify the modes that had the most prominent displacement along the z-axis of the assembly.

The random response step is a linear perturbation procedure used to predict the response of a system using non-deterministic excitation, and identifies the frequencies with the highest energy levels over a specified frequency range. The random vibration profile used in this step is based on the same NASA GEVS profile used during all experimental vibration testing of this work (revert to Section 4.4). This step was used the identify the frequencies of each assembly with the highest energy levels, in order to validate the results of the previous frequency step.

The modal dynamics step is used to analyze the transient response of a system using modal superposition ${ }^{10}$ (see Appendix A.6 for more details). This step was used to analyze the damping behavior of the three FEA test pod assemblies when perturbed by the instantaneous $1 \mathrm{~N}$ concentrated force. Similar to the experimental decay response plots (revert to Section 4.5), plots were generated from the FEA models to compare the damping behavior of the test pod, mass model, and CubeSat CPX. 


\subsubsection{Boundary Conditions}

An encastre boundary condition was applied to the bottom panel of the fixture plate to anchor the test pod FEA assembly during the vibration analysis (see Figure 5.25). This type of boundary condition prevents translational and rotational motions in all three axes, which essentially locks the bottom panel of the fixture plate. It is assumed that the fixture plate remains fixed to the moving slip plate throughout a vibrations test, so the encastre boundary condition was used to reflect this constraint throughout the three aforementioned steps created in Abaqus.

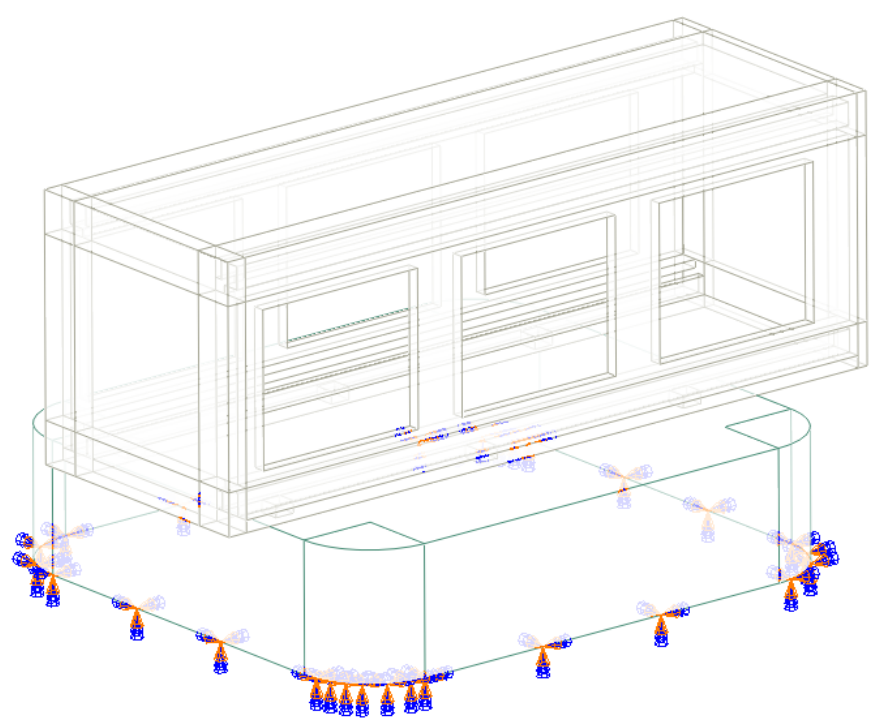

Figure 5.25: Encastre boundary condition applied to the base of the FEA fixture plate. 


\subsubsection{Supplementary FEA}

ANSYS is another commercial FEA program available for multi-physics applications where materials, boundary conditions, connections, and mesh generation are set up in a similar fashion to Abaqus. Some notable differences between the two programs however, is that ANSYS has a built-in material library, and can also keep track of the unit system being used in a model. The latter is especially beneficial, as a model built in Abaqus entrusts the user to enter values that are consistent with one unit system. ANSYS also has a default meshing tool that does not require partitions like those used in Abaqus, although ANSYS does have a manual partition tool available. This automated partition feature can save time and effort in generating an adequate mesh for an FEA body. Taking the test pod for example, the mesh generated in ANSYS (see Figure 5.26) was slightly more uniform compared to the mesh generated in Abaqus (revert to Figure 5.24), yet both performed almost identically after running a quick analysis. So while ANSYS was not the main FEA program used for the dynamic analysis of the test pod assembly, it did serve to verify the preliminary models built in Abaqus in an effort to further validate the FEA setups.

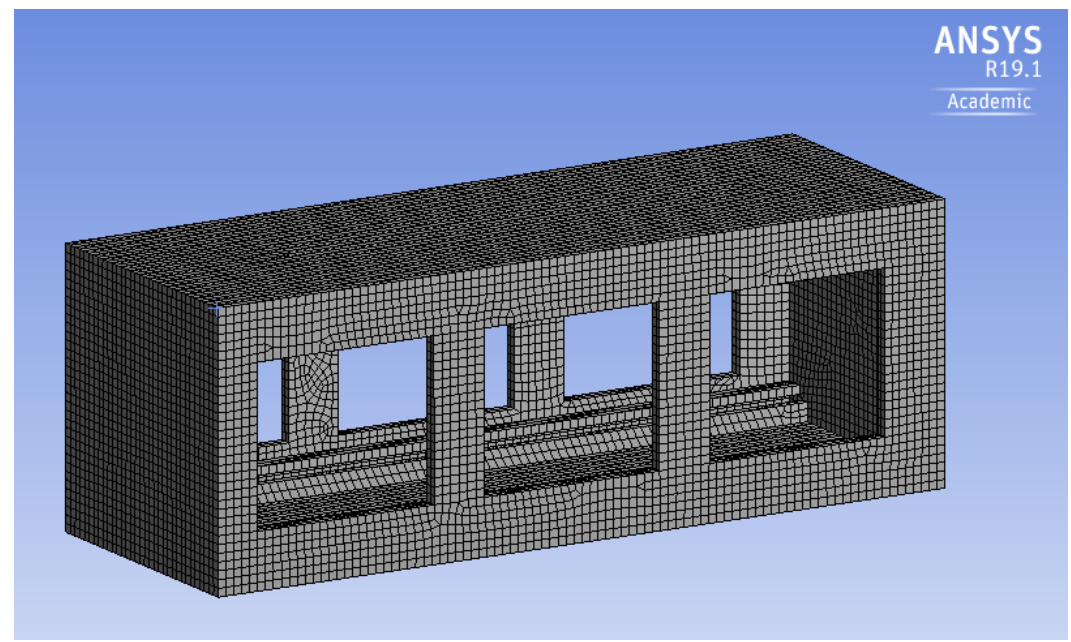

Figure 5.26: ANSYS model of the test pod with an auto-partitioned mesh. 


\subsection{Preliminary Modeling}

\subsubsection{Cantilever Slender Beam}

A $0.010 \mathrm{~m} \times 0.10 \mathrm{~m} \times 1 \mathrm{~m}$ aluminum cantilever slender beam was modeled in Abaqus and ANSYS, and results were then compared to the classical beam theory solution using Matlab. From Table 5.2, it is evident that the first two natural frequencies of both FEA programs converge to the classic solution.

Table 5.2: Mode 1 and 2 of a cantilever slender beam.

\begin{tabular}{|c|c|c|c|}
\hline Mode & $\begin{array}{c}\text { Abaqus } \\
(\mathbf{H z})\end{array}$ & $\begin{array}{c}\text { ANSYS } \\
(\mathbf{H z})\end{array}$ & $\begin{array}{c}\text { Classic Beam Theory } \\
(\mathbf{H z})\end{array}$ \\
\hline 1 & 8.24 & 8.24 & 8.18 \\
\hline 2 & 51.62 & 51.61 & 51.25 \\
\hline
\end{tabular}
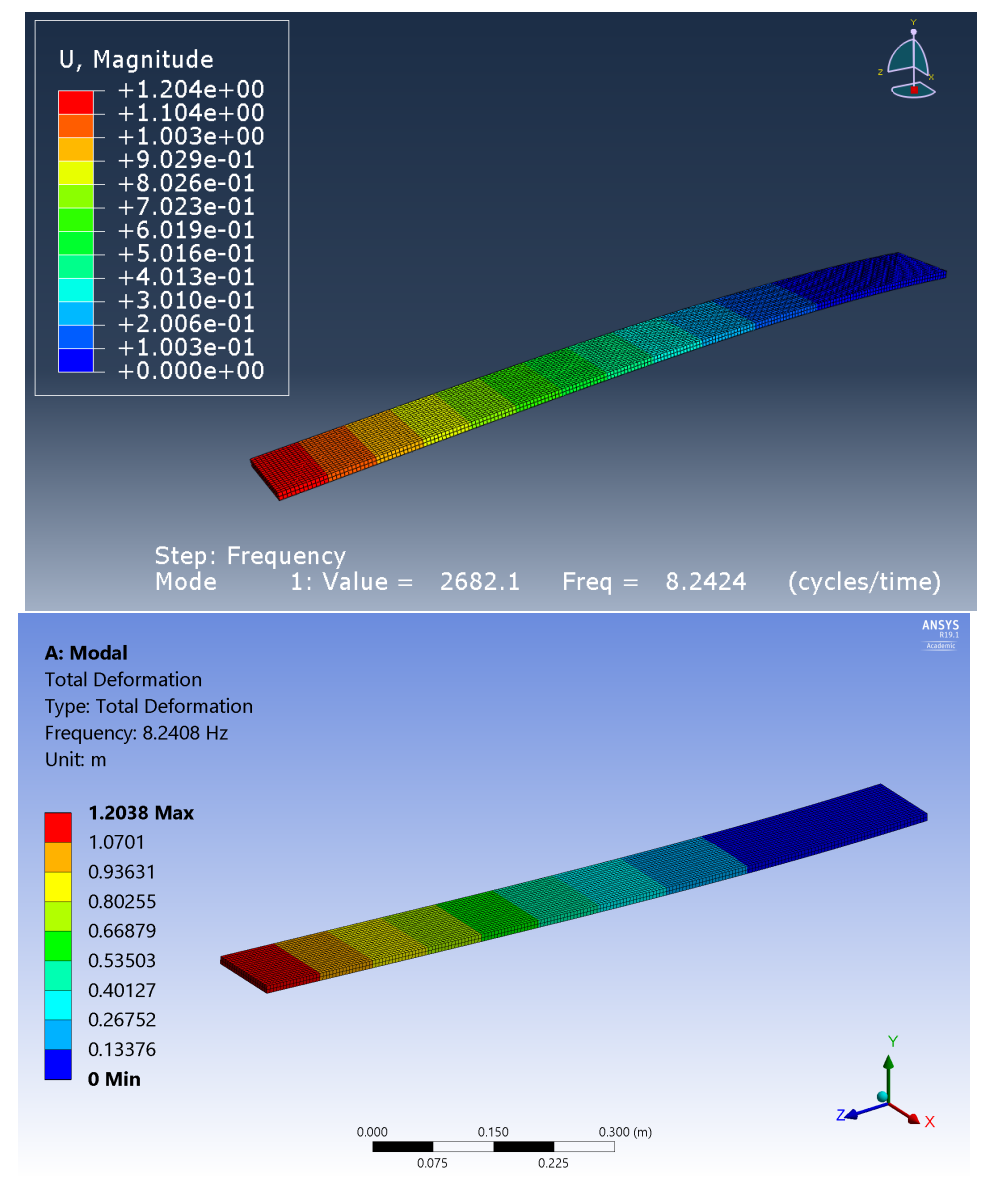


\subsubsection{Cantilever Solid Square Beam}

Resembling a long and solid test pod, a $0.1295 \mathrm{~m}$ x $0.1295 \mathrm{~m}$ x $1.295 \mathrm{~m}$ aluminum cantilever solid square beam was modeled in Abaqus and ANSYS, and results were then compared to the classical beam theory solution using Matlab. From Table 5.3, it is evident that the first natural frequency of both FEA programs converge to the classic solution.

Table 5.3: Mode 1 of a cantilever solid square beam.

\begin{tabular}{|c|c|c|c|}
\hline Mode & $\begin{array}{c}\text { Abaqus } \\
(\mathrm{Hz})\end{array}$ & $\begin{array}{c}\text { ANSYS } \\
(\mathrm{Hz})\end{array}$ & $\begin{array}{c}\text { Classic Beam Theory } \\
(\mathrm{Hz})\end{array}$ \\
\hline 1 & 63.06 & 63.02 & 63.15 \\
\hline
\end{tabular}
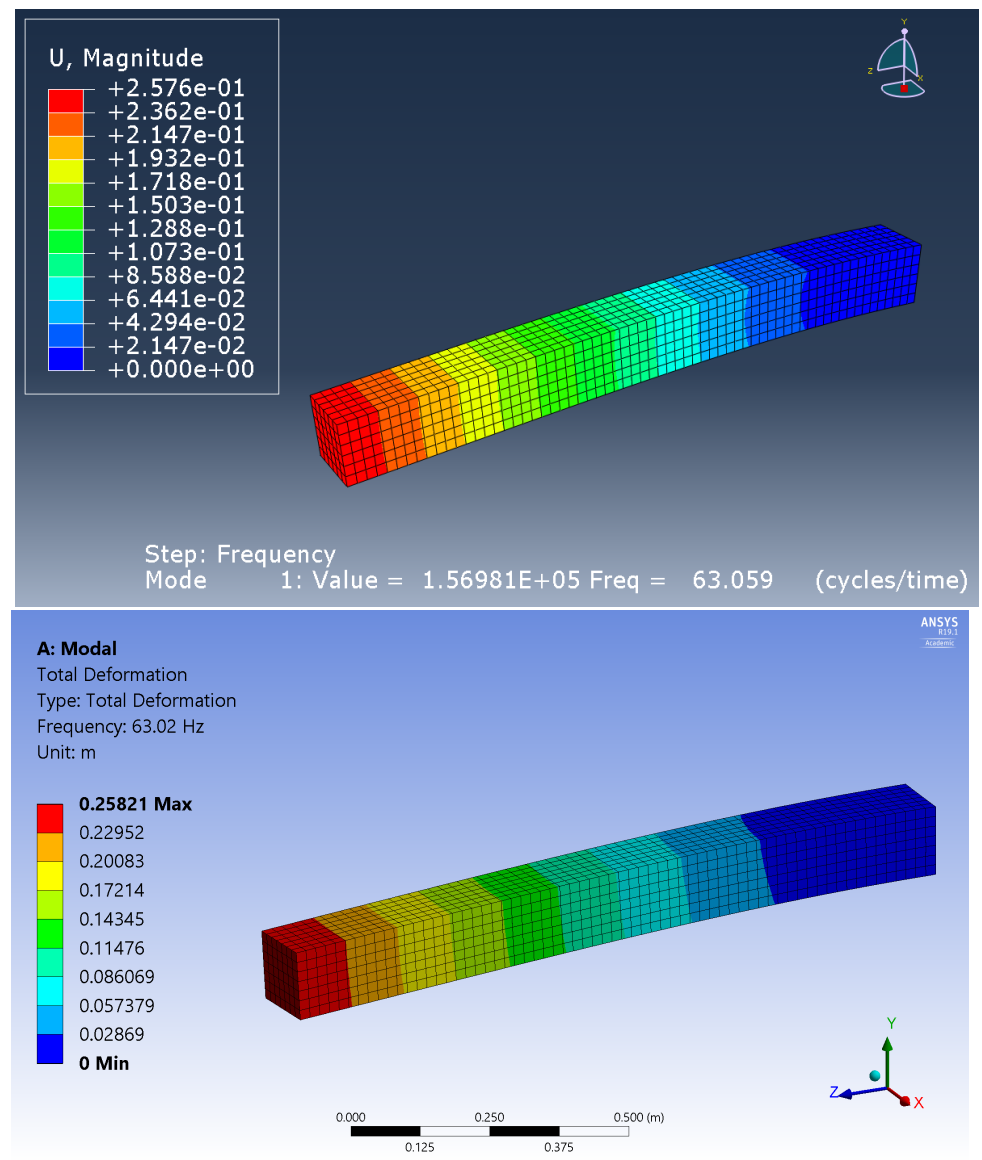


\subsubsection{Cantilever Hollow Square Beam}

Resembling a long test pod, a $0.1295 \mathrm{~m}$ x $0.1295 \mathrm{~m}$ x $1.295 \mathrm{~m}$ aluminum cantilever hollow square beam with a wall thickness of $0.0059 \mathrm{~m}$ was modeled in Abaqus and ANSYS, and results were then compared to the classical beam theory solution using Matlab. From Table 5.4, it is evident that the first natural frequency of both FEA programs converge near the classic solution. Note that although the classic solution assumes open ends, the approximation for a closed body is still fairly accurate.

Table 5.4: Mode 1 of a cantilever hollow square beam.

\begin{tabular}{|c|c|c|c|}
\hline Mode & $\begin{array}{c}\text { Abaqus } \\
(\mathrm{Hz})\end{array}$ & $\begin{array}{c}\text { ANSYS } \\
(\mathrm{Hz})\end{array}$ & $\begin{array}{c}\text { Classic Beam Theory } \\
(\mathrm{Hz})\end{array}$ \\
\hline 1 & 80.34 & 80.31 & 85.33 \\
\hline
\end{tabular}

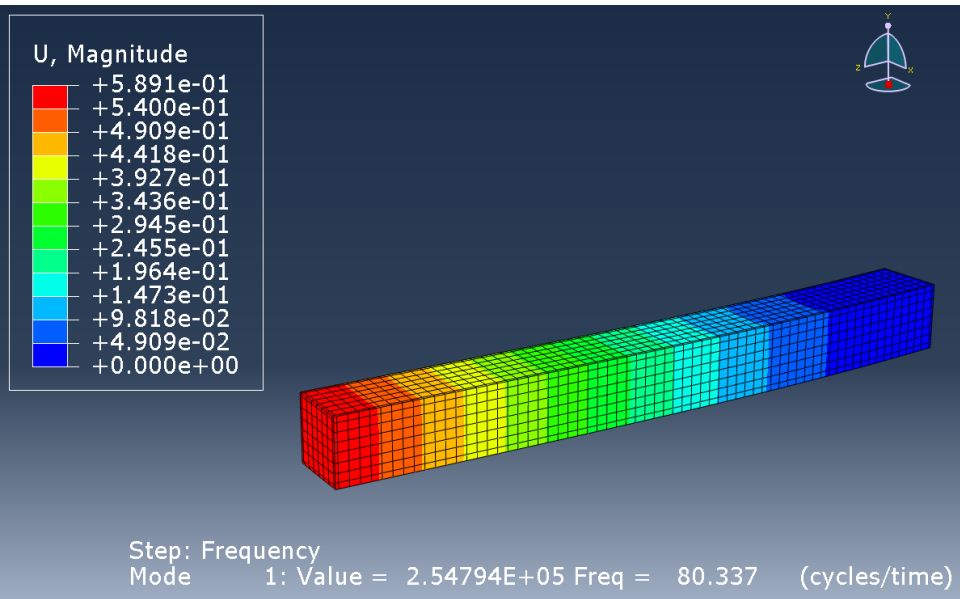

A: Modal

Total Deformation

Type: Total Deformation

Frequency: $80.311 \mathrm{~Hz}$

Unit: $m$
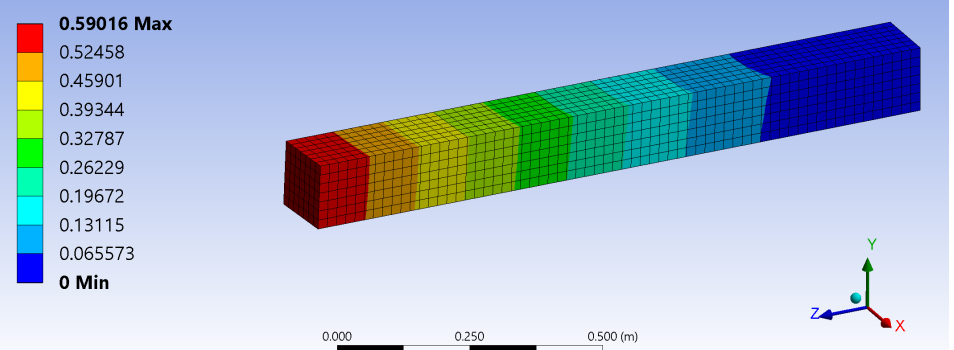

$0.000 \quad 0.250 \quad 0.500(\mathrm{~m})$ 


\subsection{Complete FEA assembly of the Test Pod and Mass Model}

With preliminary models cross-checked and validated, an added level of confidence was established in the FEA setup of the test pod assembly. With all test parameters set in the FEA model, the meshed test pod, mass model, and fixture plate were assembled into the final FEA assembly (see Figure 5.27). The frequency, random response, and modal dynamics steps were executed, with the final results to be discussed in Chapter 6.

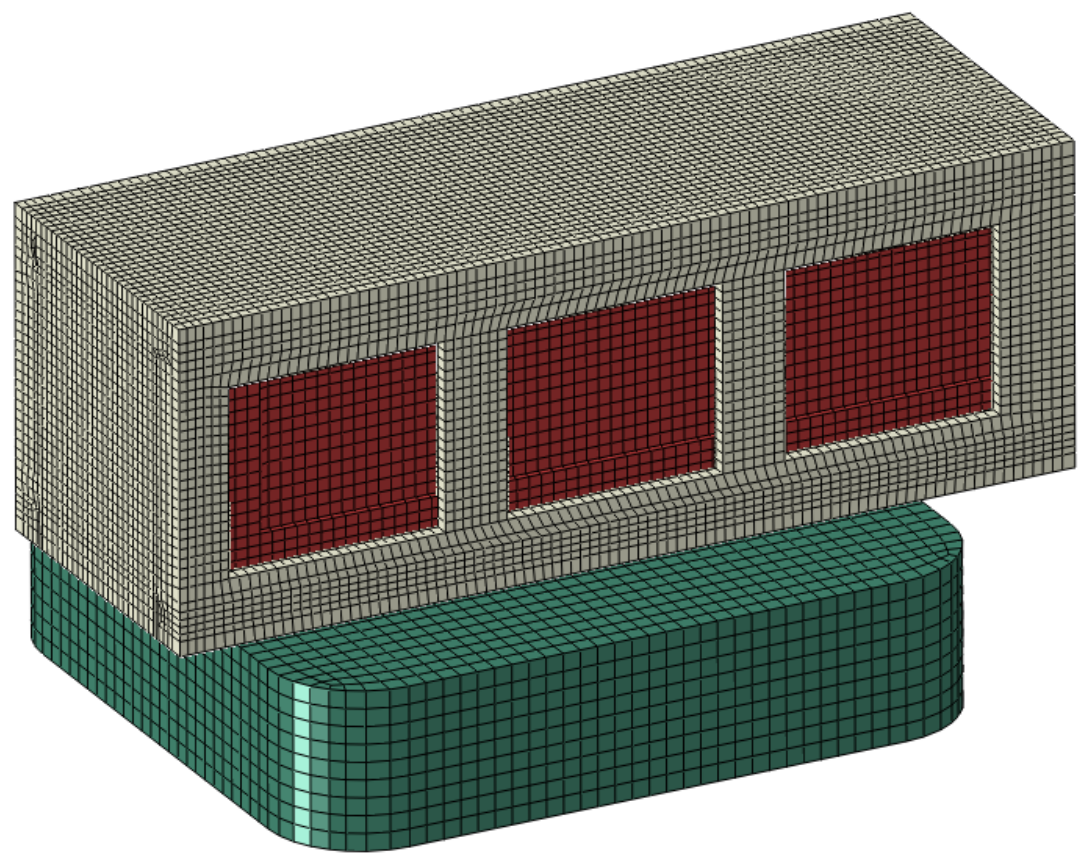

Figure 5.27: Meshed FEA assembly of the test pod and mass model. 


\subsection{Complete FEA assembly of the Test Pod and CubeSat CPX}

Intricate geometry features of a body can add significant processing time to an FEA program, as finer elements are required to properly dicretize such features, which in turn increases the number of elements of the body and thus the number of calculations. In order to simplify the analysis of CPX, all screw holes and fillets were removed from the CAD assembly before importing to Abaqus (see Figure 5.28). Such features also complicate the partitions that need to be created to achieve an adequate mesh, and so omitting them allowed the generation of a relatively uniform mesh, which is generally ideal in FEA modeling.
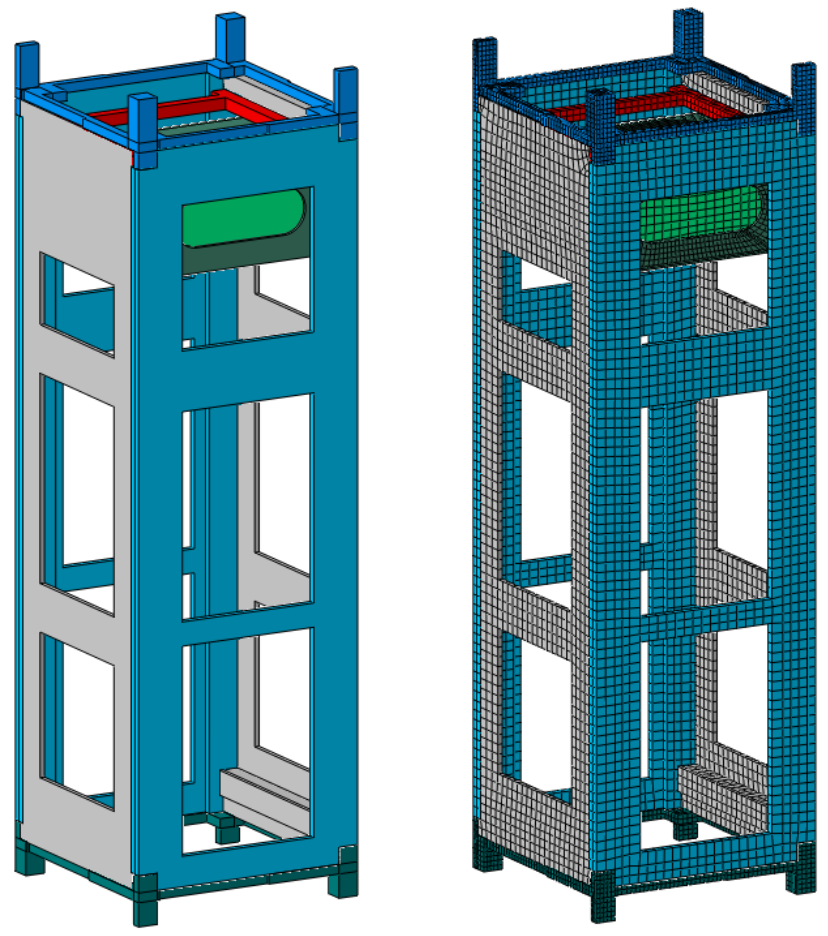

Figure 5.28: Simplified CPX structure (left) and mesh generation (right).

In order to stabilize the vibrations of the thin mid-section of the CPX structure, a block was added to the FEA assembly to represent a payload (see Figure 5.29). Also, batteries add significant mass to a CubeSat structure, and so they were modeled as 
one piece (green) held within the battery brackets of the structure. As most CubeSat structures are made from aluminum, these same material properties were applied to the payload block and battery piece for modeling purposes.

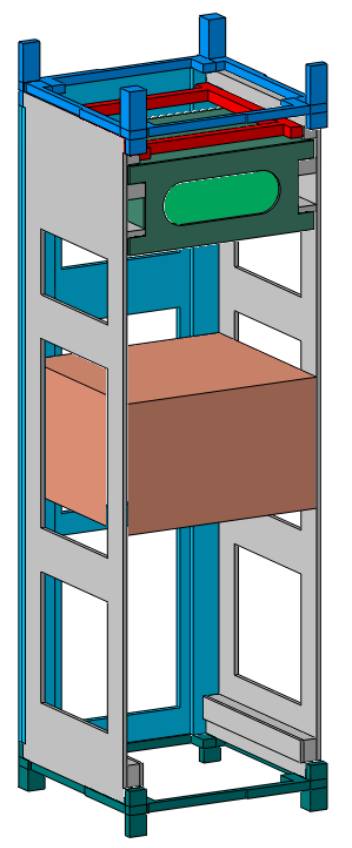

Figure 5.29: Payload block is used to add rigidity to the CPX structure.

The interactions, constraints, boundary conditions, and other FEA test parameters where set in the same manner as the FEA assembly of the loaded test pod, but in this case replacing the mass model with the CPX structure (see Figure 5.30). However, as CPX is assembled from several components, the structure was first constrained to form one single assembly before constraining it to the test pod. Nodal ties were used only at contact surfaces that were assumed to have the highest clamping force (i.e. fastener areas). Otherwise, using nodal ties at all contact surfaces of CPX would make the structure too stiff and add error to the FEA simulation. Completing the full FEA assemblies for two CPX configurations (see Figure 5.30 and 5.31), the same frequency, random response, and modal dynamics steps were executed, with all FEA results to be discussed next in Chapter 6 . 


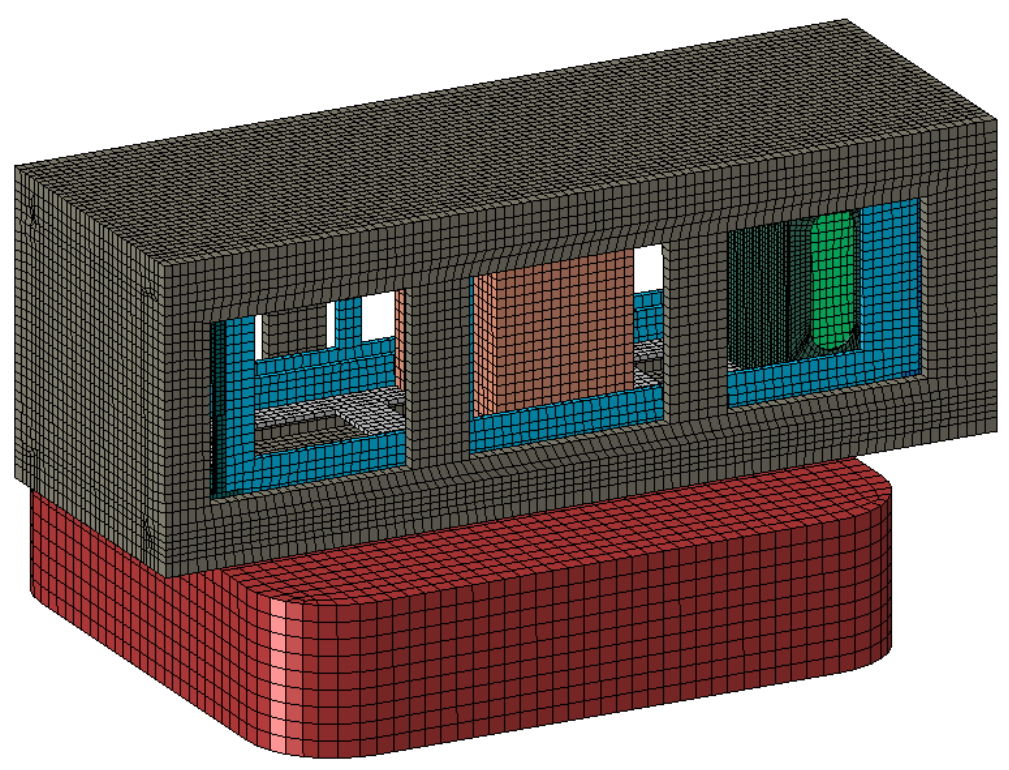

Figure 5.30: Meshed FEA assembly of the test pod and CubeSat CPX.

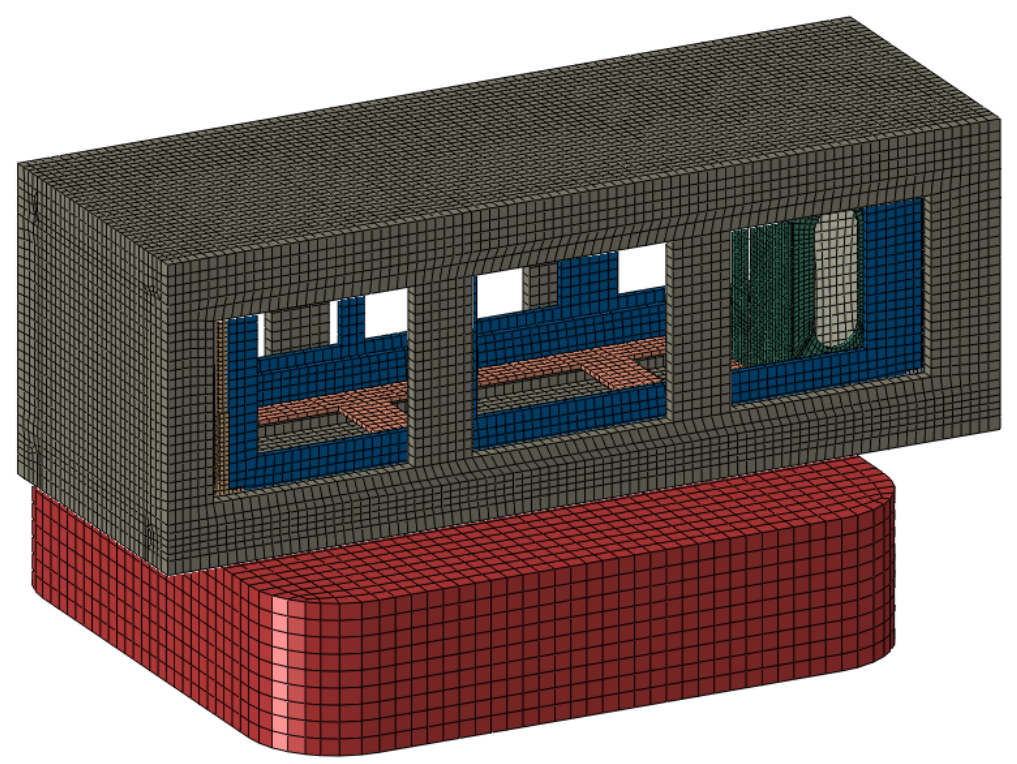

Figure 5.31: Meshed FEA assembly of the test pod and CubeSat CPX (no payload). 
Chapter 6

FEA RESULTS AND THEIR COMPARISON TO EXPERIMENTAL DATA

\subsection{Results of the FEA models}

To validate the FEA models, experimental results obtained from the vibration tests were compared to the FEA results. It was necessary to first analyze the results for the test pod and mass model, as this was the experimental data gathered first hand. In addition, since the CPX FEA assembly is based on the test pod/mass model FEA template, the final evaluation of the CPX structure design depended highly on the FEA performance of the test pod and mass model.

The FEA natural frequencies, mode shapes (see Figure 6.1), and transient free response will first be discussed for the test pod, mass model, and CPX. These results will then be compared to experimental results, followed by a final evaluation of the CPX structure design for proof of concept.

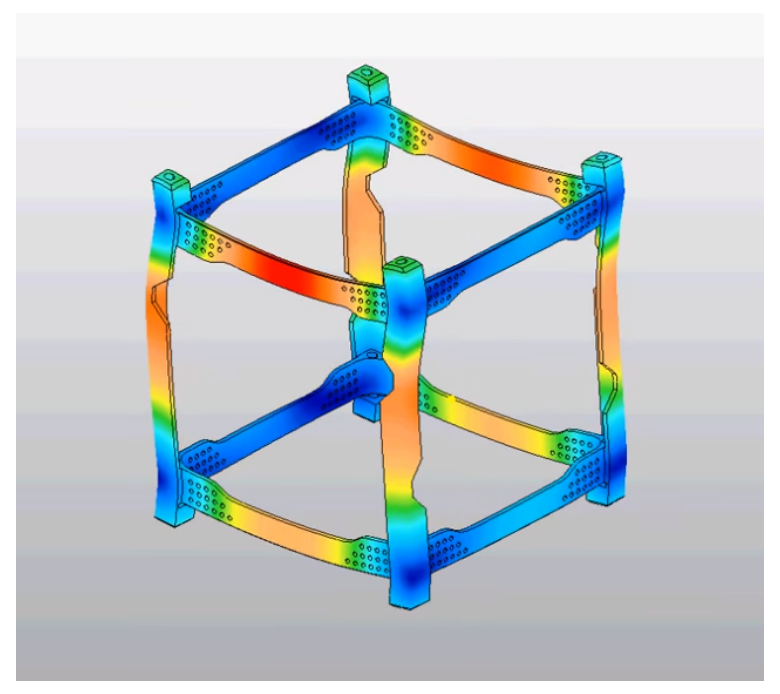

Figure 6.1: Example of an FEA mode shape for a 1U CubeSat frame. ${ }^{30}$ 


\subsubsection{Natural Frequencies}

\subsubsection{Test Pod}

From the frequency step, the first seven modes and natural frequencies were successfully extracted from the empty test pod FEA assembly (see Figure 6.2). The number of modes was limited to the range of $2000 \mathrm{~Hz}$, as this is generally the upper limit of vibration tests performed for CubeSats.

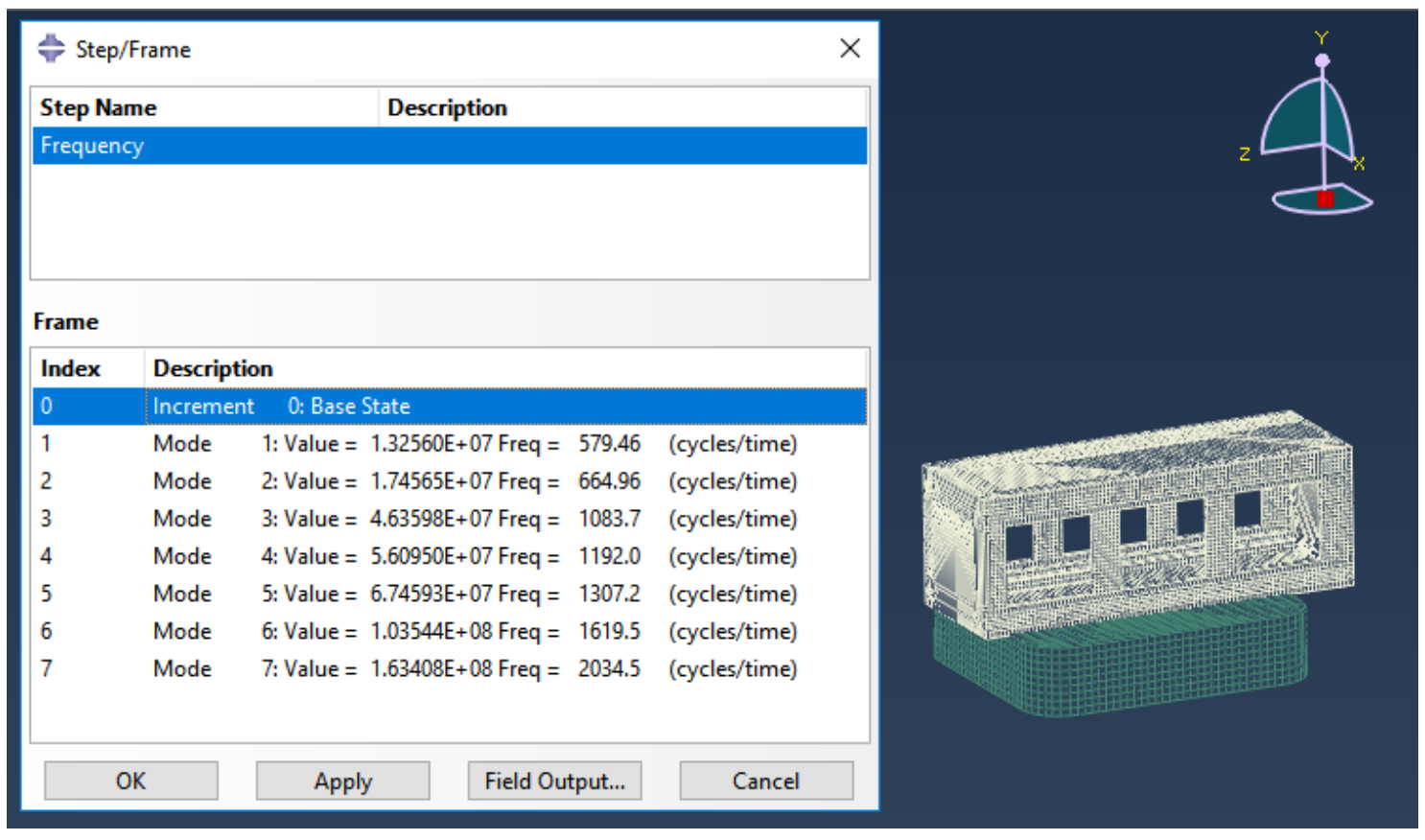

Figure 6.2: Natural frequencies of the empty test pod FEA assembly.

It should be noted that the natural frequencies depend on the nature of the entire assembly, and not solely on the response of the test pod alone. As proof, two separate studies were performed to test this conclusion. In the first study, the fixture plate was removed entirely from the FEA assembly. In the second study, the test pod was constrained to the fixture plate using the full bottom panel of the test pod, instead of the small screw partitions. From Table 6.1, it is clear that the fixture plate and the 
type of constraint used between the test pod and fixture plate both have a significant effect on the system response.

Table 6.1: Comparison of natural frequencies in the two studies performed for the empty test pod FEA assembly.

\begin{tabular}{|c|c|c|c|}
\hline Mode & $\begin{array}{c}\text { Current model } \\
(\mathbf{H z})\end{array}$ & $\begin{array}{c}\text { Study 1 } \\
\text { No fixture plate } \\
(\mathbf{H z})\end{array}$ & $\begin{array}{c}\text { Study 2 } \\
\text { Bottom panel of test pod } \\
\text { fully constrained } \\
(\mathbf{H z})\end{array}$ \\
\hline 1 & 579 & 608 & 774 \\
\hline 2 & 665 & 681 & 782 \\
\hline 3 & 1084 & 1097 & 1167 \\
\hline 4 & 1192 & 1270 & 1549 \\
\hline 5 & 1307 & 1328 & 1560 \\
\hline 6 & 1620 & 1636 & 1859 \\
\hline 7 & 2035 & 2117 & 2356 \\
\hline
\end{tabular}

Due to the overhangs of the test pod over the fixture plate, these sections are not as rigid and can instead behave as a set of "cantilever" beams, which makes them more susceptible to modes of vibration. This may help explain why the system responds to lower frequencies with the added fixture plate, as seen in Table 6.1.

Constraining the entire bottom panel of the test pod adds stiffness to the test pod, which increases the frequency response of the test pod. Using such a constraint would be more in line with a welded or "fused" assembly, which is not the case in the experimental setup. So, the structural nature of the thin test pod makes it sensitive to other components in the assembly, and to the type of constraints being used. 


\subsubsection{Test Pod with Mass Model}

From the frequency step, the first nine modes and natural frequencies were successfully extracted from the loaded test pod FEA assembly (see Figure 6.3), again limiting the number of modes based on the frequency limit of vibration tests for CubeSats.

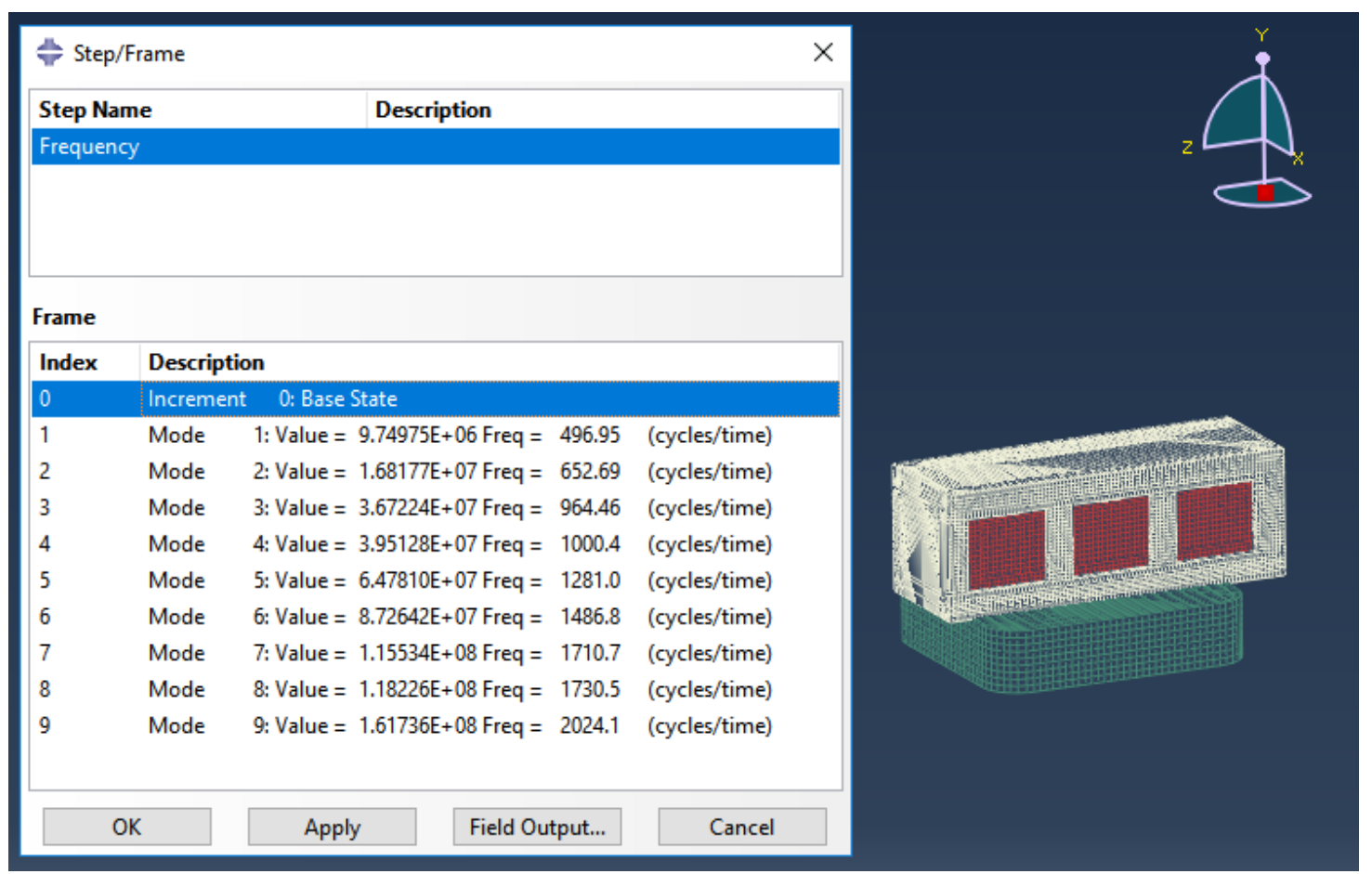

Figure 6.3: Natural frequencies of the test pod with mass model FEA assembly.

Comparing this set of natural frequencies with those of Figure 6.2, the system now responds to lower natural frequencies due in part to the added weight of the mass model that has been fixed to the test pod (recall $\omega_{n}=\sqrt{k / m}$ ). Similar to the empty test pod assembly, removing the fixture plate from the model or altering the constraint of the test pod would affect the response of the loaded test pod assembly. 


\subsubsection{Test Pod with CubeSat CPX}

One of the first notable differences between CPX and the mass model is that CPX has significantly more natural frequencies according to the FEA model (see Figure 6.4). Recall that the mass model CAD was designed as one solid body, unlike the CPX structure which is assembled using several components. Multiple components in an assembly adds degrees of freedom to the system, which in turn increases its sensitivity to dynamic excitation. In addition, the mass distribution of the CPX structure and payload also affects the response, as opposed to the symmetric and solid mass model.

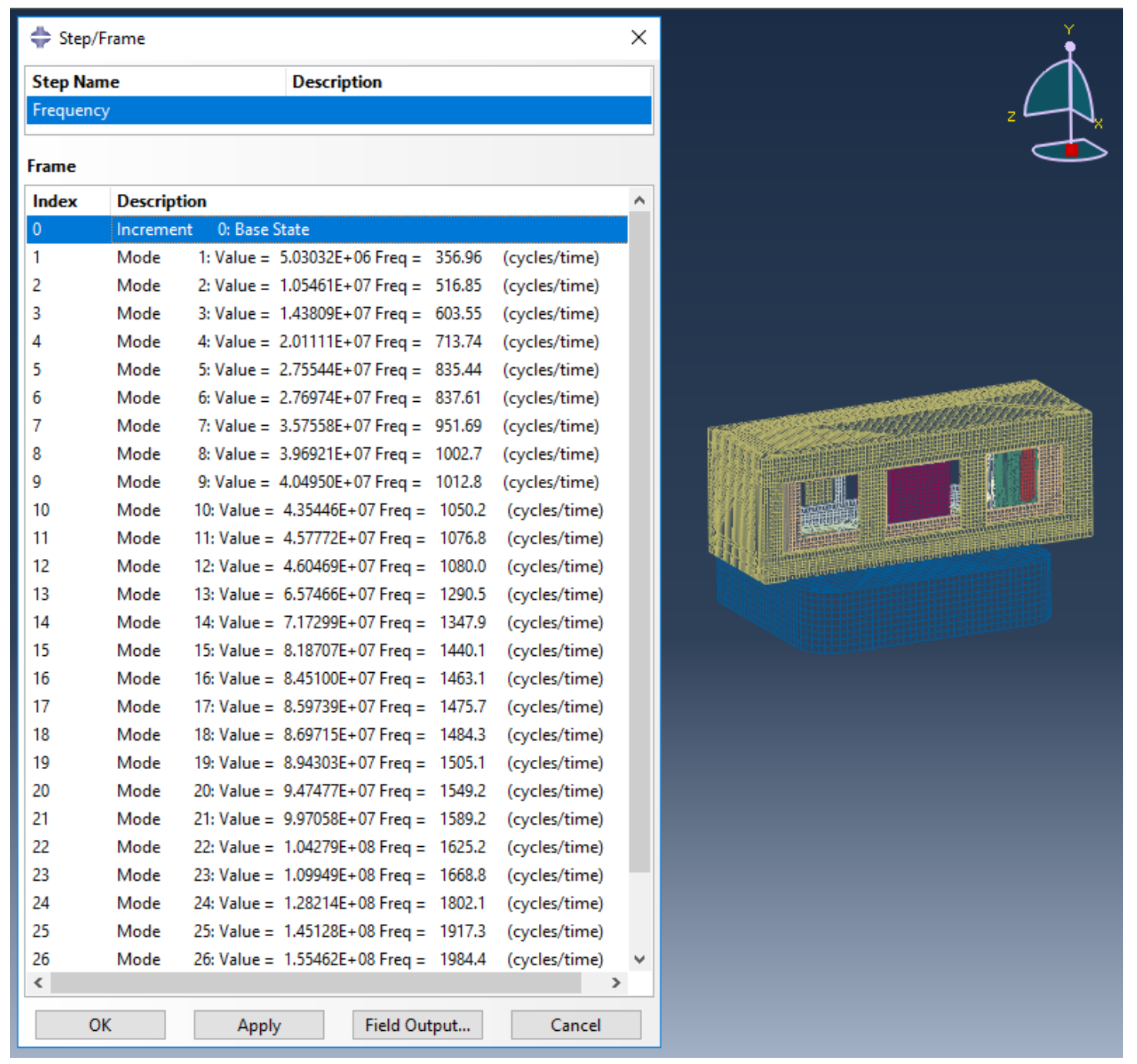

Figure 6.4: Natural frequencies of the test pod with CPX FEA assembly. 
Removing the payload block from the CPX structure, the FEA model shows an excitation of additional modes in the structure for the same frequency range (see Figure 6.5). This is due in part to the thin walls of the structure mid section, as the absence of a payload no longer supports these areas, making them more susceptible to deformation. Without the added support of a payload, additional degrees of freedom at the mid section add natural frequencies to the overall CPX structure.

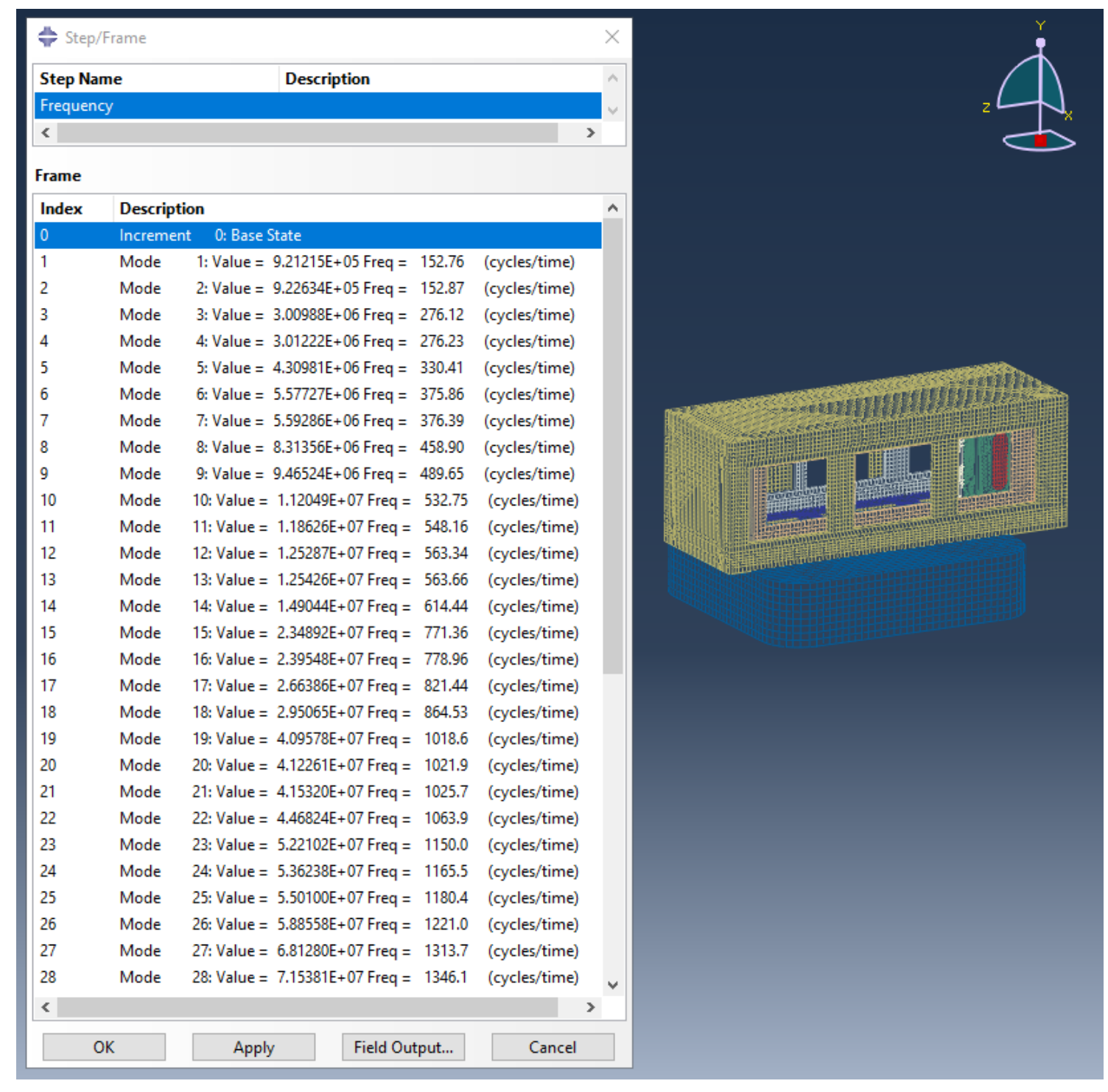

Figure 6.5: Natural frequencies of the test pod with CPX (no payload) FEA assembly. 


\subsubsection{Mode Shapes}

\subsubsection{Test Pod}

From the random response step of the empty test pod FEA assembly, the second mode of vibration at $665 \mathrm{~Hz}$ has the largest acceleration spectral density (ASD) along the zaxis (see Figure 6.6). In other words, mode 2 has the largest energy level in the z-axis for the specified frequency spectrum. Furthermore, using the mode shape tool of the natural frequency step, mode 2 at $664.96 \mathrm{~Hz}$ has the largest displacement magnitude in the z-axis (see Figure 6.7), which agrees with the results of the random response step. Since mode 2 has the most prominent effect along the z-axis (compared to the other six modes), it is expected that the empty test pod would see a peak response near $665 \mathrm{~Hz}$ when excited in the z direction.

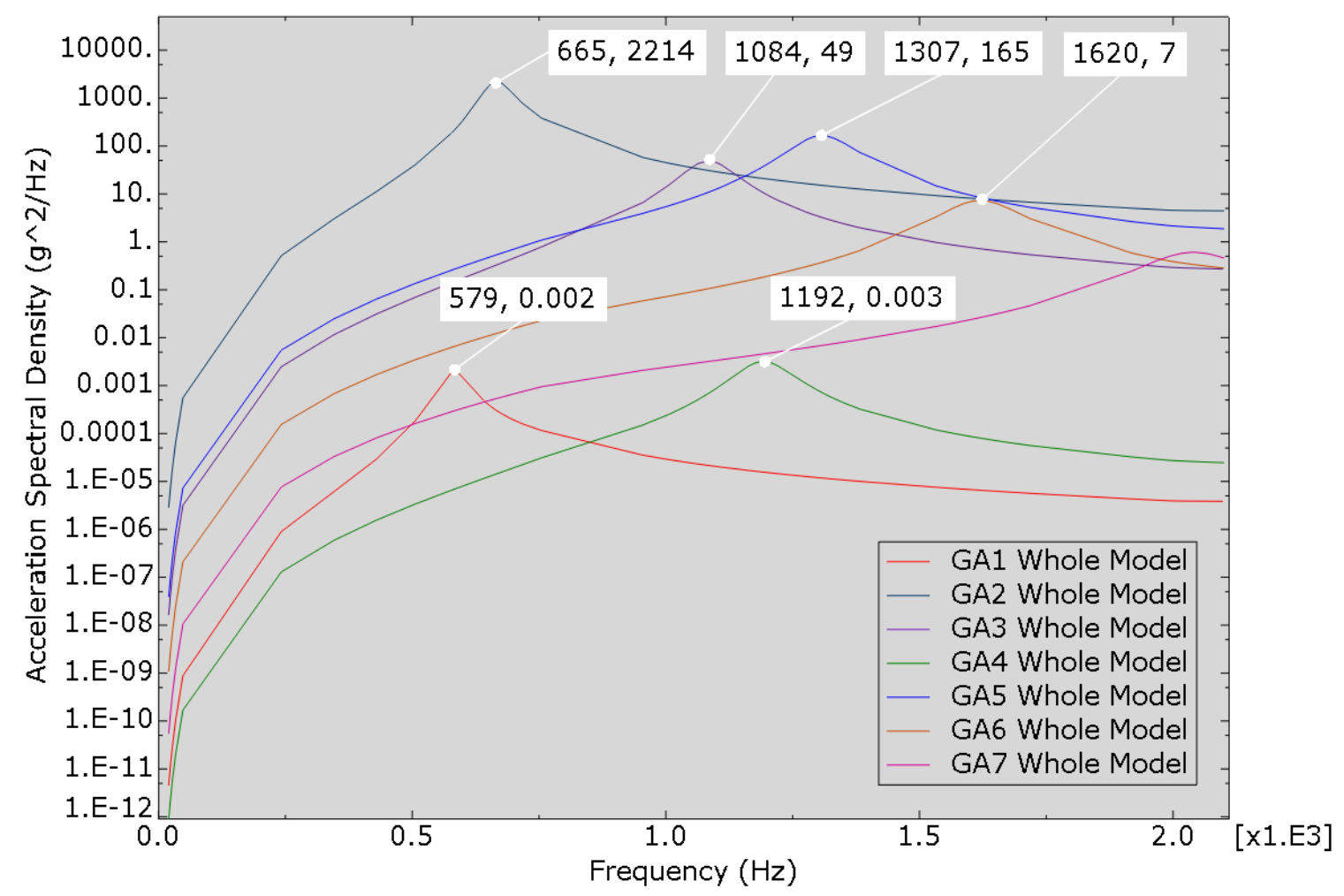

Figure 6.6: General acceleration (GA) of Mode $2(665 \mathrm{~Hz})$ has the highest ASD response in the z-axis of the empty test pod FEA assembly. 


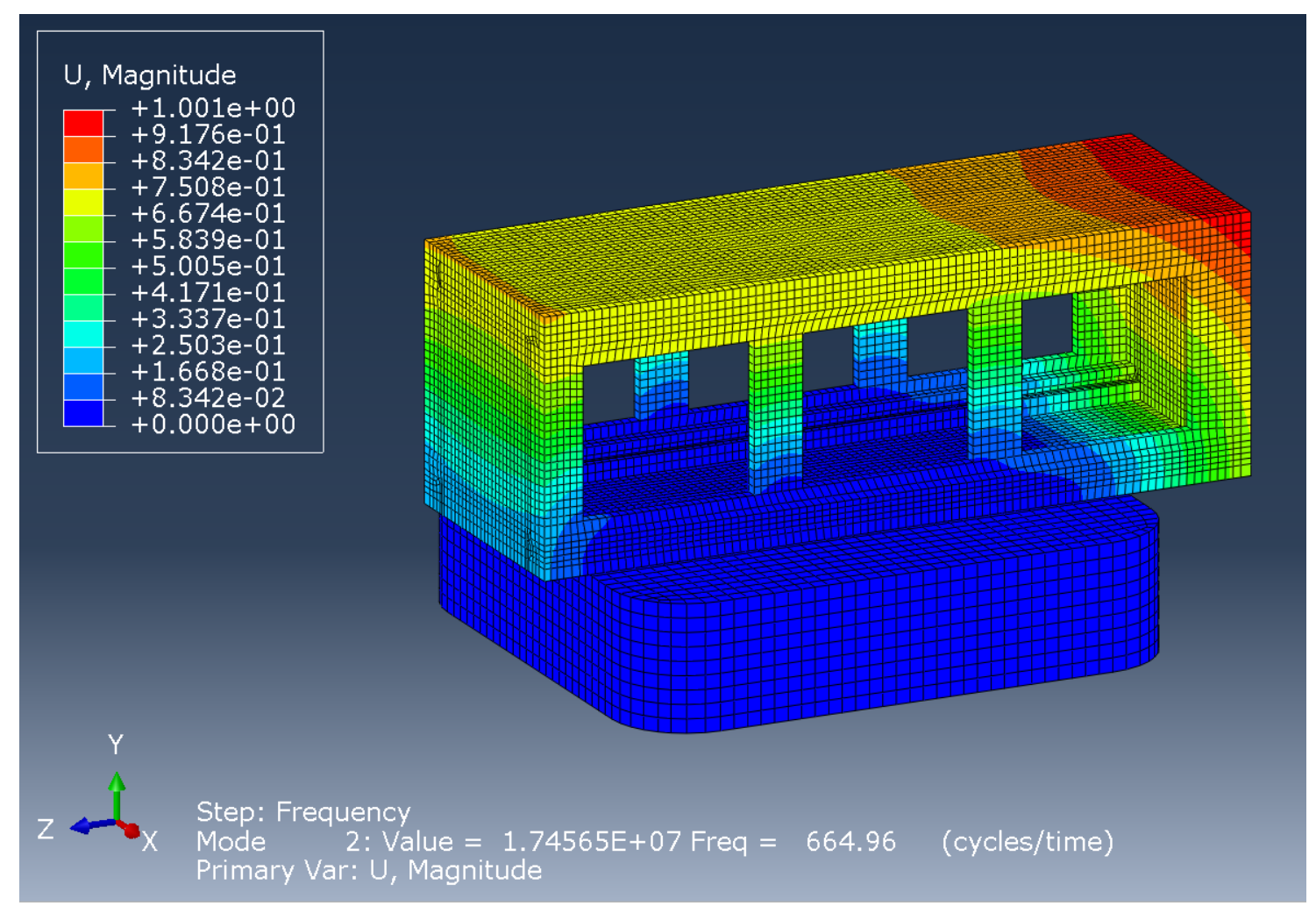

Figure 6.7: Mode $2(664.96 \mathrm{~Hz})$ amplifies the displacement (U) in the z-axis of the empty test pod FEA assembly.

From Figure 6.7, note that the displacement is more pronounced (red elements) on the side where the test pod overhang is large. The lack of support at this end of the test pod makes it more susceptible to deformation, while areas fully supported by the fixture (blue elements) have minimal deformation, if any. 


\subsubsection{Test Pod with Mass Model}

From the random response step of the loaded test pod FEA assembly, the first mode of vibration at $497 \mathrm{~Hz}$ has the largest ASD along the z-axis (see Figure 6.8). Note that with the mass model, the prominent mode of vibration along the $\mathrm{z}$ axis shifts from Mode 2 to Mode 1 when compared to the response of the empty test pod. Furthermore, using the mode shape tool, mode 1 at $496.95 \mathrm{~Hz}$ has the largest displacement magnitude in the z-axis (see Figure 6.9), which agrees with the results of the random response step. Since mode 1 has the most prominent effect along the z-axis (compared to the other eight modes), it is expected that the loaded test pod would see a peak response near $497 \mathrm{~Hz}$ when excited in the z direction.

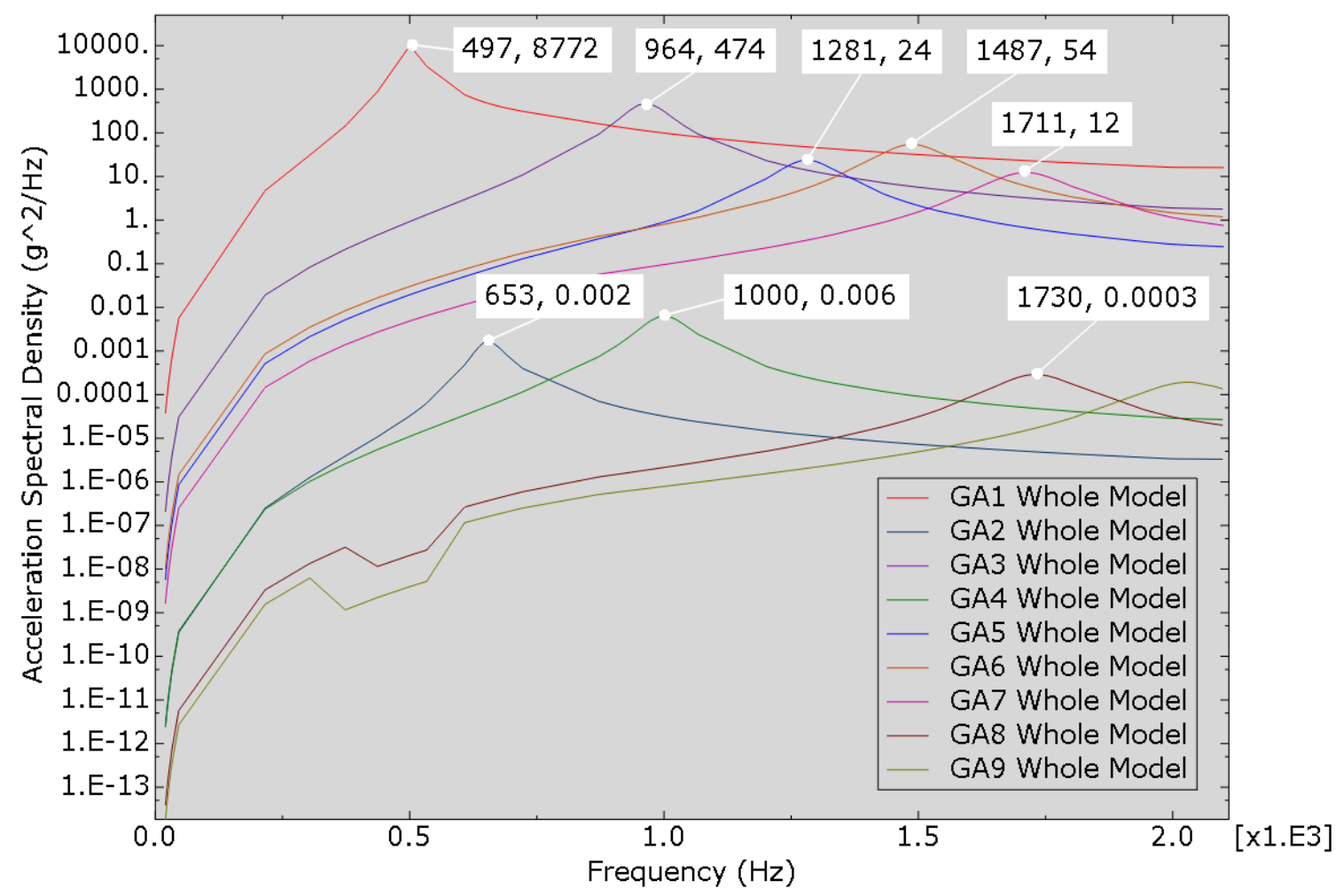

Figure 6.8: General acceleration (GA) of Mode $1(497 \mathrm{~Hz})$ has the highest ASD response in the z-axis of the test pod with mass model FEA assembly. 


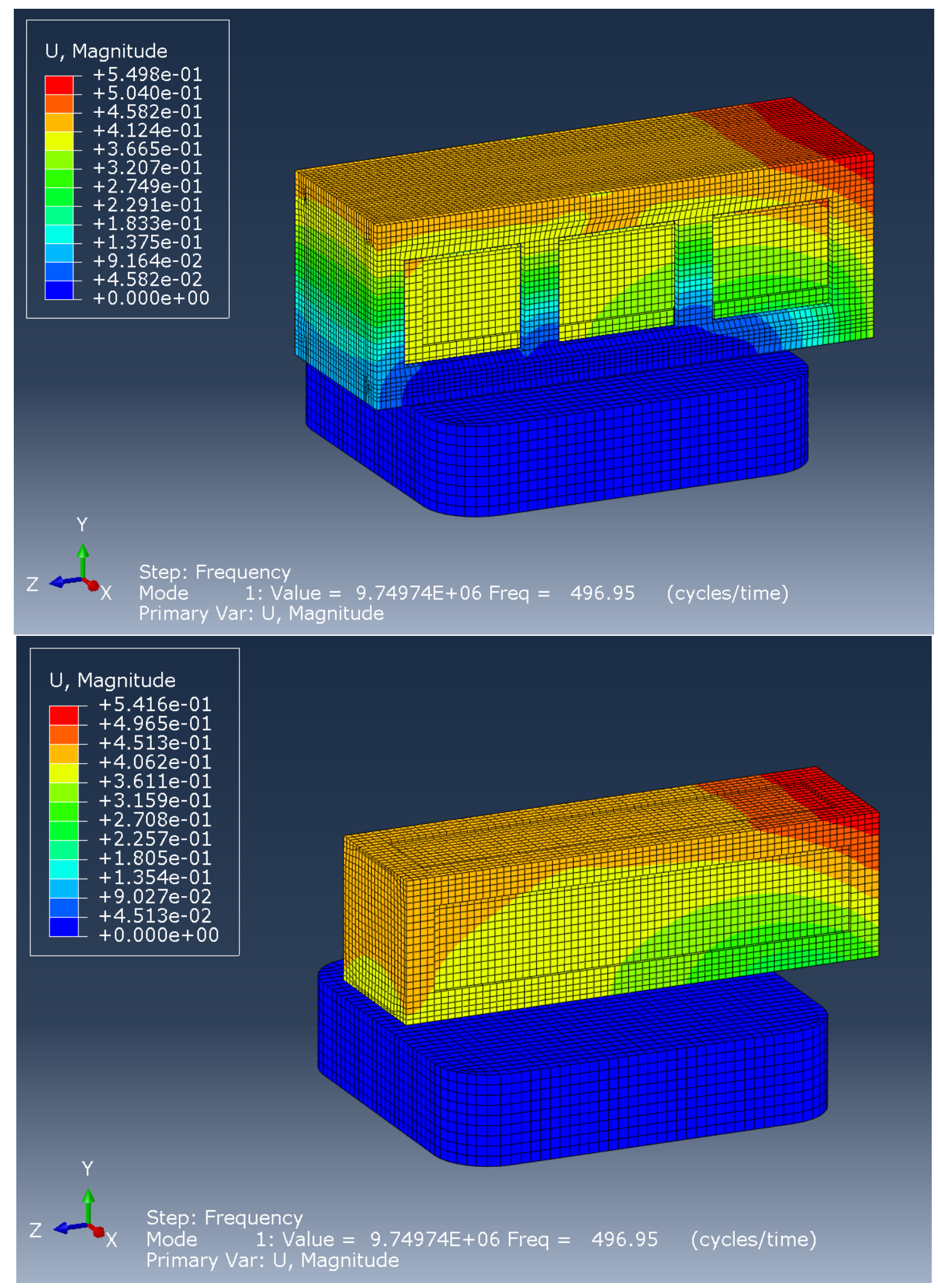

Figure 6.9: Mode $1(496.95 \mathrm{~Hz})$ amplifies the displacement $(\mathrm{U})$ in the z-axis of the test pod with mass model FEA assembly. 


\subsubsection{Test Pod with CubeSat CPX}

From the random response step of the test pod and CPX FEA assembly, the second mode of vibration at $517 \mathrm{~Hz}$ has the largest ASD along the z-axis (see Figure 6.10). Note that with CPX, the prominent mode of vibration along the $\mathrm{z}$ axis is the same as the empty test pod, unlike with the mass model where it changed to mode 1 . Using the mode shape tool, mode 2 at $516.85 \mathrm{~Hz}$ has the largest displacement magnitude in the z-axis (see Figure 6.11), which agrees with the results of the random response step. Since mode 2 has the most prominent effect along the z-axis (compared to the other eight modes), it is expected that the test pod and CPX assembly would see a peak response near $517 \mathrm{~Hz}$ when excited in the z direction. Although only the first 9 modes were plotted in Figure 6.10, all other modes from the frequency step $(+20$ modes) were inspected to determine that the largest peaks exist within these 9 modes.

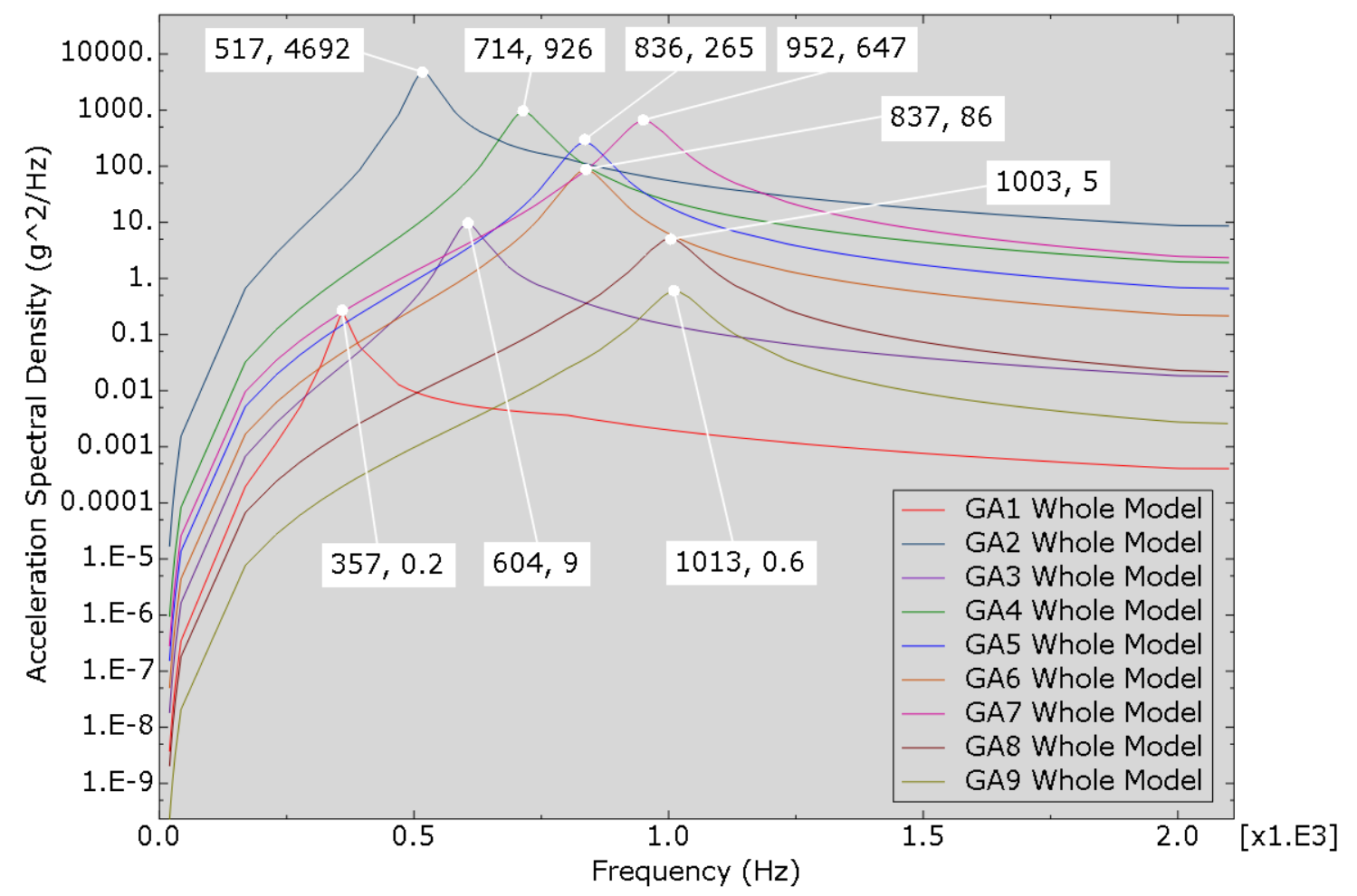

Figure 6.10: General acceleration (GA) of Mode $2(517 \mathrm{~Hz})$ has the largest ASD response in the z-axis of the test pod with CPX FEA assembly. 


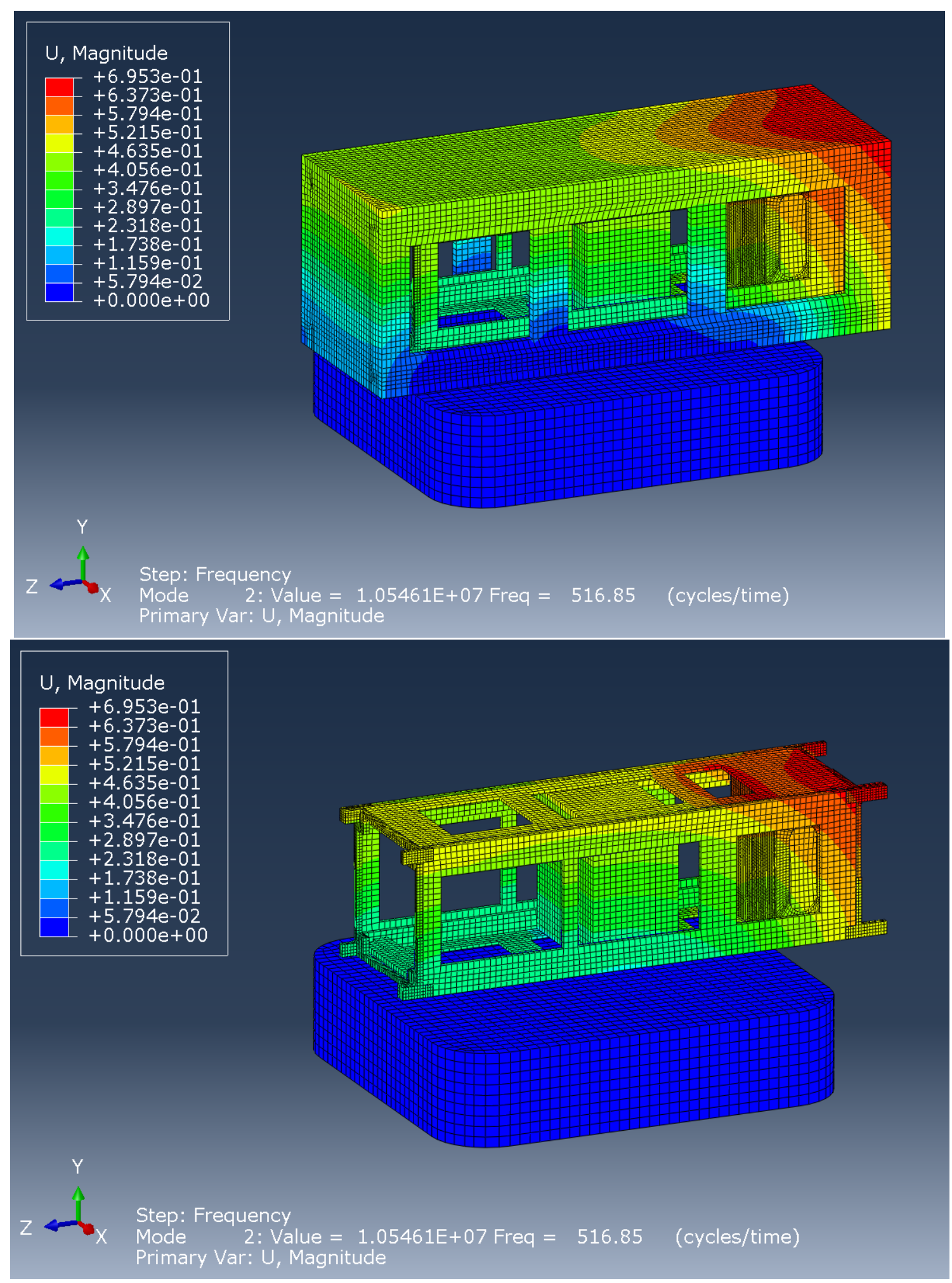

Figure 6.11: Mode $2(516.85 \mathrm{~Hz})$ amplifies the displacement $(\mathrm{U})$ in the z-axis of the test pod and CPX FEA assembly. 
For the second configuration of CPX (no payload), mode 11 at $549 \mathrm{~Hz}$ now has the largest ASD along the z-axis (see Figure 6.12). Note that without a payload, the prominent mode of vibration along the $\mathrm{z}$ axis "shifts" to the right from mode 2 to 11. With the mode shape tool, mode 11 at $548.16 \mathrm{~Hz}$ has the largest displacement magnitude in the z-axis (see Figure 6.13), which agrees with the results of the random response step. Since mode 11 has the most prominent effect along the z-axis, it is expected that the test pod and CPX (no payload) assembly would see a peak response near $548 \mathrm{~Hz}$ when excited in the z direction. Although only the first 12 modes were plotted in Figure 6.12, all other modes from the frequency step (+20 modes) were inspected to determine that the largest peaks exist within these 12 modes.

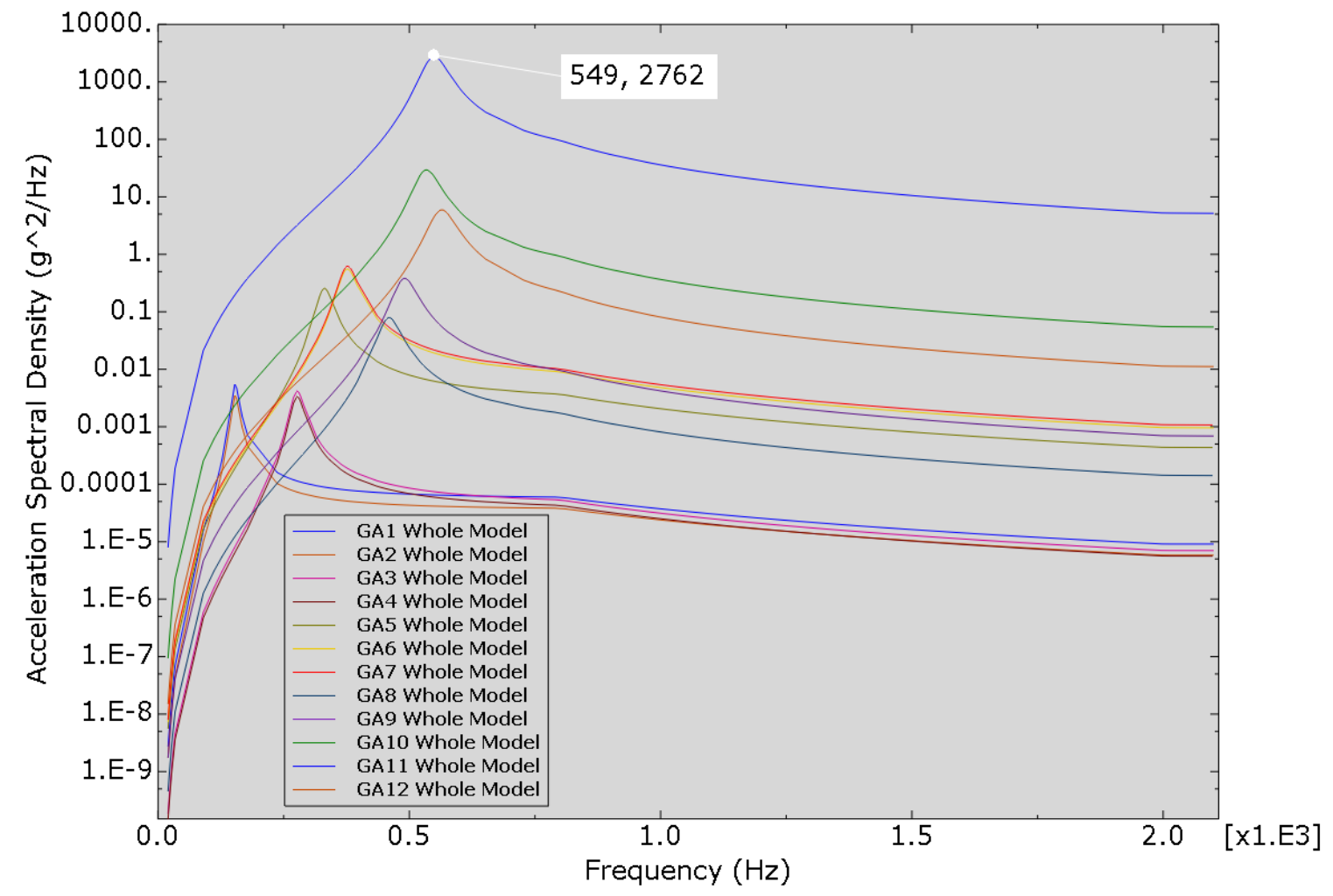

Figure 6.12: General acceleration (GA) of Mode $11(549 \mathrm{~Hz})$ has the largest ASD response in the z-axis of the test pod with CPX (no payload) FEA assembly. 

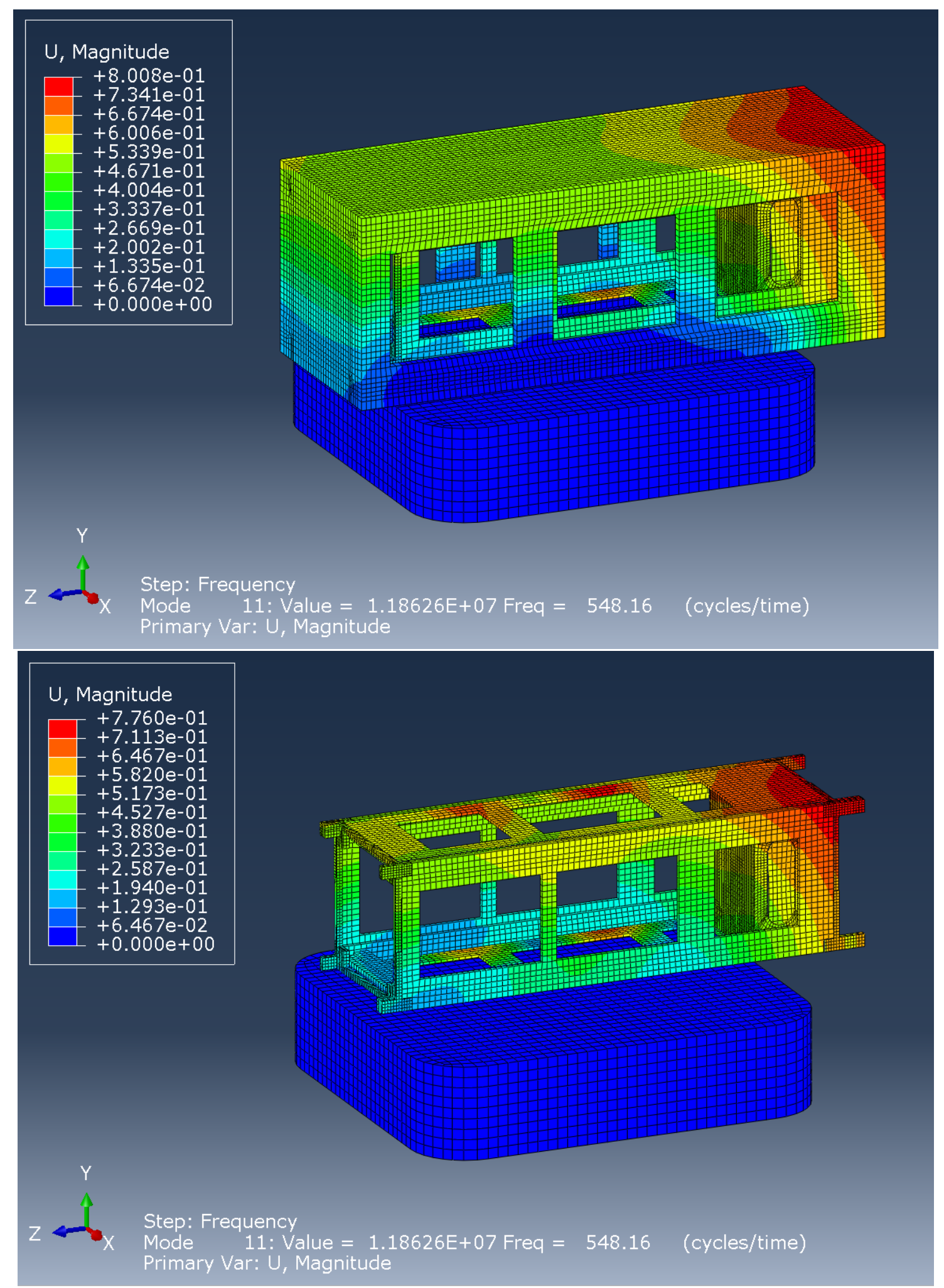

Figure 6.13: Mode $11(548.16 \mathrm{~Hz})$ amplifies the displacement (U) in the z-axis of the test pod and CPX (no payload) FEA assembly. 
From Figure 6.11 and 6.13, the effects of vibration are evident on the CPX structure. The thin panel areas near the battery pack, along with the stand-off protrusions (red elements), have the largest displacements. Therefore, any sensitive equipment installed on or near these areas would see the highest levels of deformation and stress. Further analysis and testing would be recommended to ensure that the integrity and function of such equipment remain intact at the natural frequencies highlighted here for the CPX concept design.

It should be made clear that the test pod, mass model, and fixture are proven structures, and their levels of deformation are small enough to not raise concern. However, CPX is not a proven structure, and the results in this section are meant to serve as starting points for further evaluation of the design. 


\subsubsection{Damped Response}

\subsubsection{Test Pod}

From the modal dynamics step with an applied $1 \mathrm{~N}$ instantaneous load (+z direction) at the top edge of the test pod assembly (red dot in Figure 6.14), note how stresses are propagated throughout the assembly. As expected, the highest levels of stress for a lateral load are found at the weakest points of the structure, which are the four vertical "beams" of the test pod.

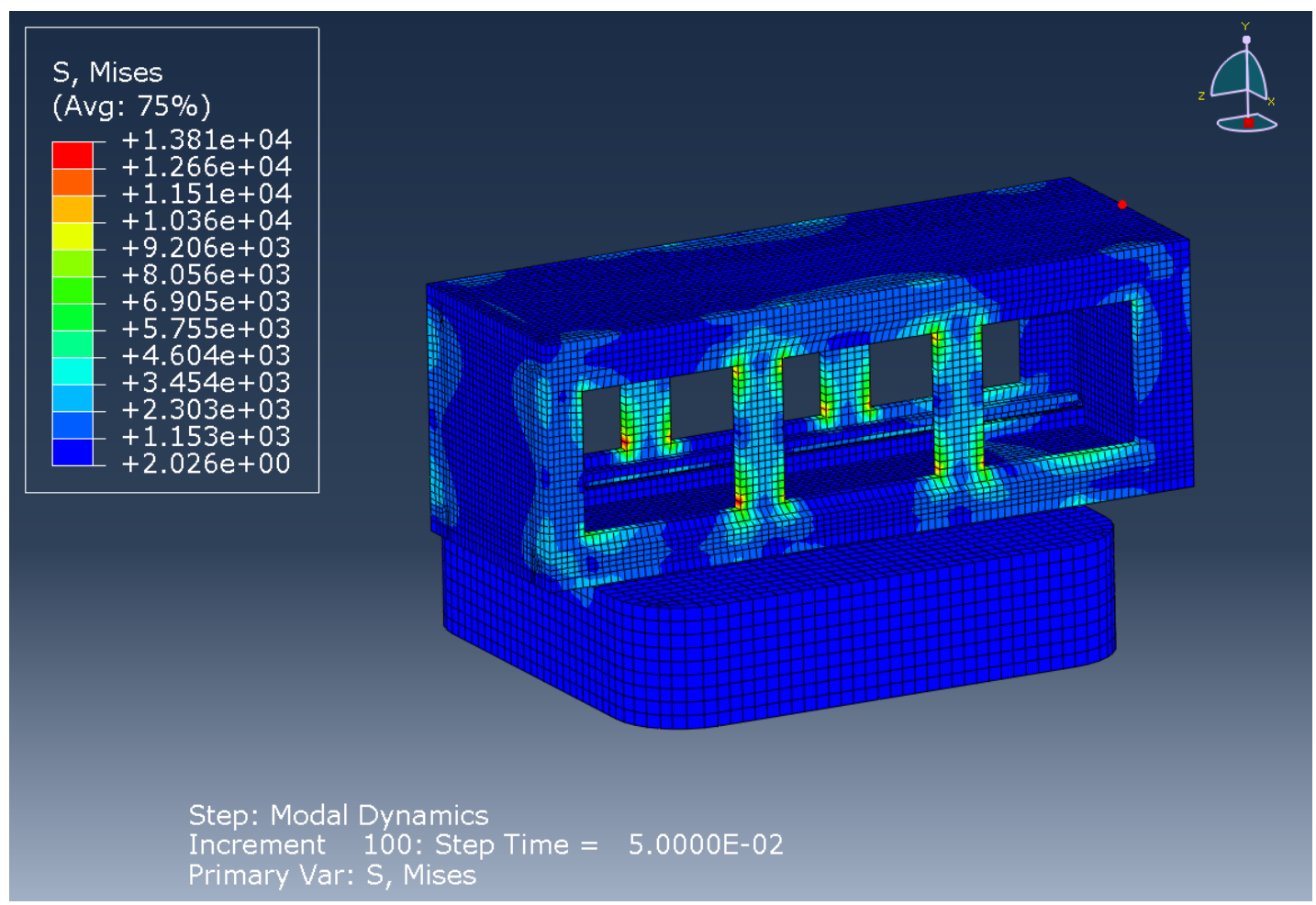

Figure 6.14: Stress areas of the empty test pod under a $1 \mathrm{~N}$ lateral load.

Plotting the transient response of the first mode, the empty test pod assembly decays exponentially and exhibits an underdamped behavior (see Figure 6.15). The stress distribution on the bottom panel of the test pod is also depicted in Figure 6.16, outlining the larger levels of stress at the screw and fixture overhang areas. 


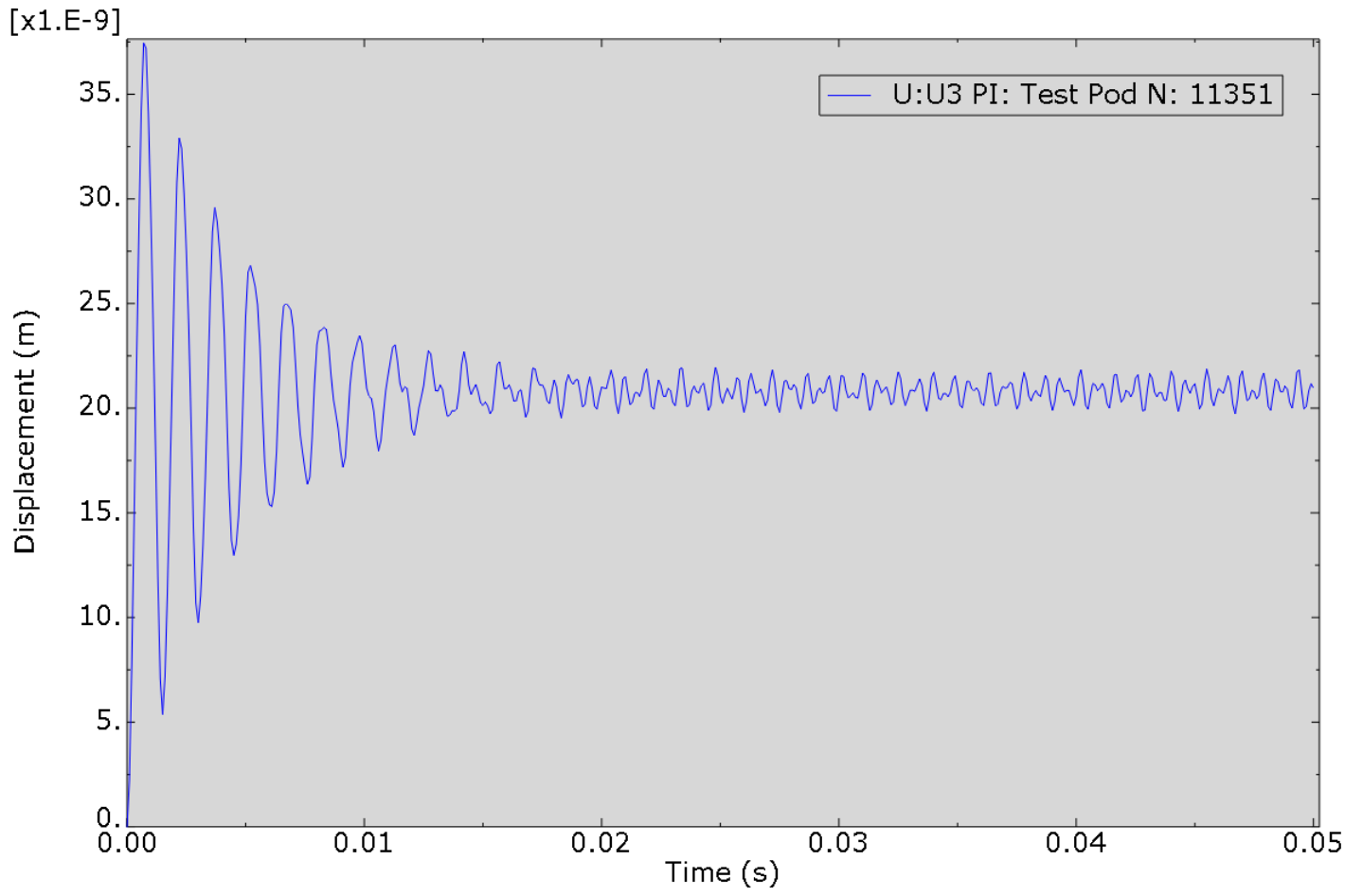

Figure 6.15: Underdamped response of the empty test pod FEA assembly.

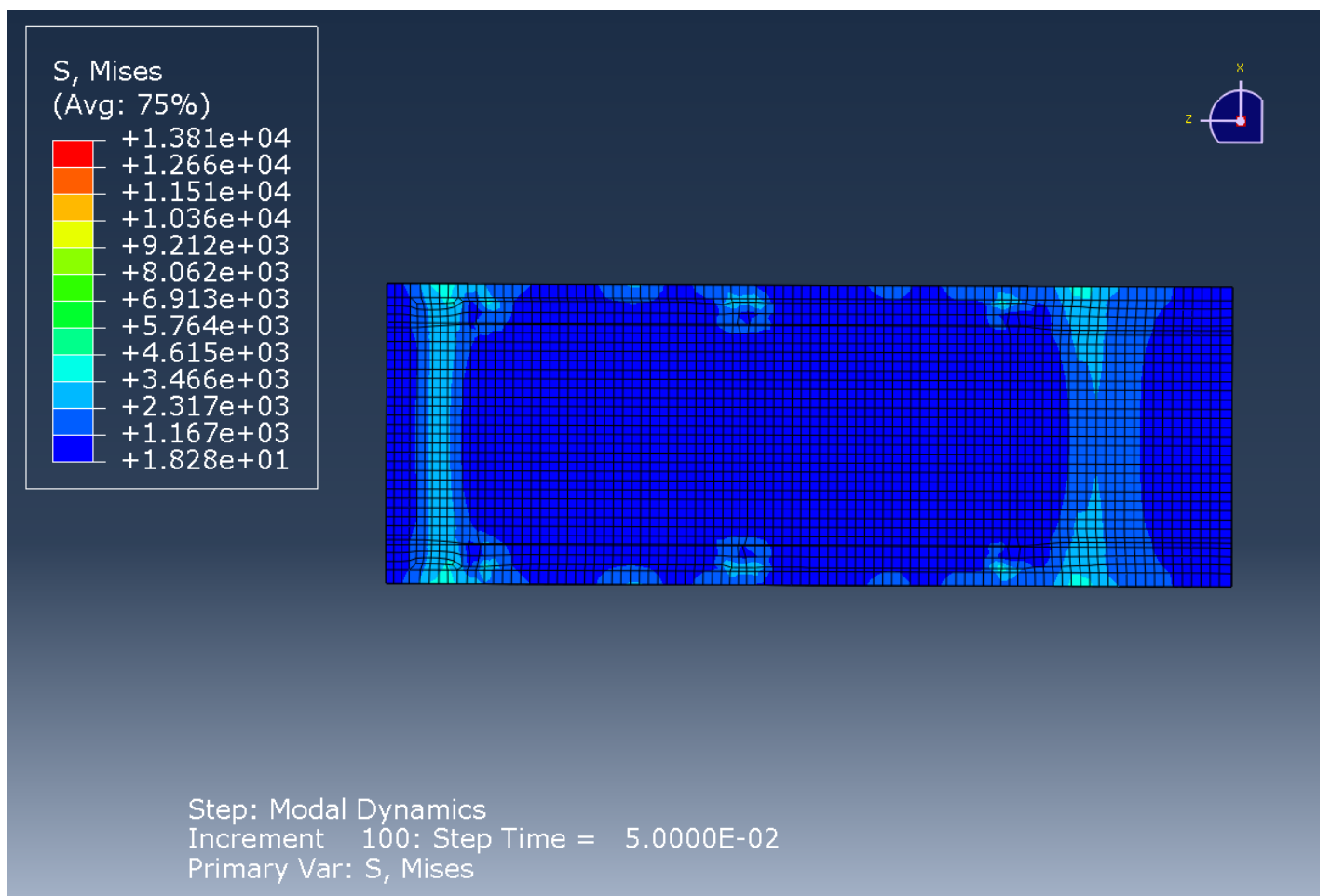

Figure 6.16: Stresses at the six screw locations and overhang areas of the empty test pod assembly. 


\subsubsection{Test Pod with Mass Model}

Applying the same $1 \mathrm{~N}$ load, the stress distribution stays roughly the same when compared to the empty test pod assembly (see Figure 6.17). This is expected as the force being applied to the assembly is relatively small.

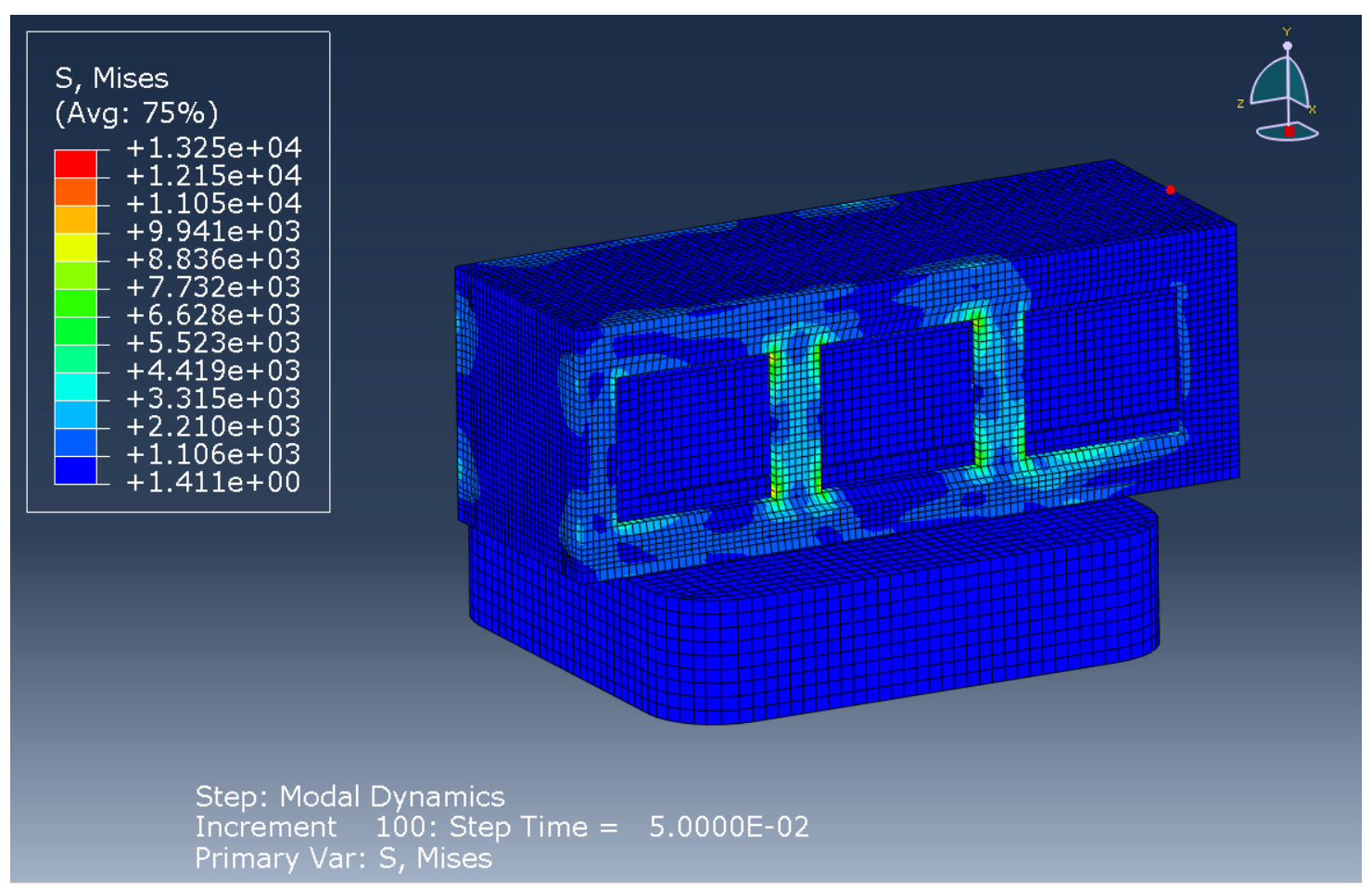

Figure 6.17: Stress areas of the test pod with mass model FEA assembly.

Like the empty test pod assembly, the transient response of the assembly exhibits an underdamped behavior (see Figure 6.18). Note that the peak amplitudes of the test pod are less pronounced than the mass model, due to inherent damping of the different materials and assembly constraints. Stresses around the screw areas are also concentrated (see Figure 6.19), and such a load would tend to "peel back" the test pod from the fixture plate. So the two screws closest to the load would take a significant part of the initial loading, as depicted on the right side of Figure 6.19. 


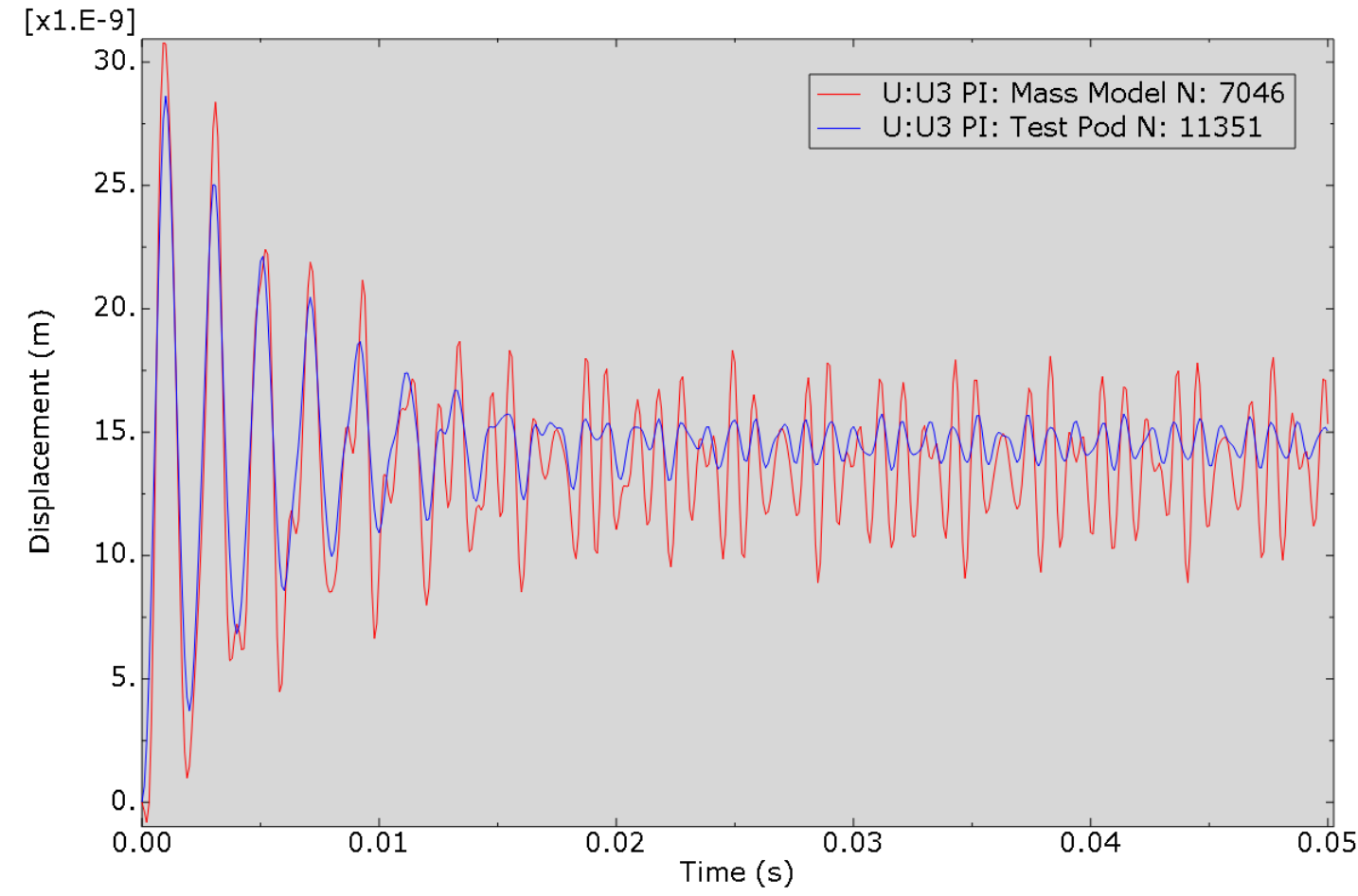

Figure 6.18: Underdamped response overlay of the test pod with mass model FEA assembly.

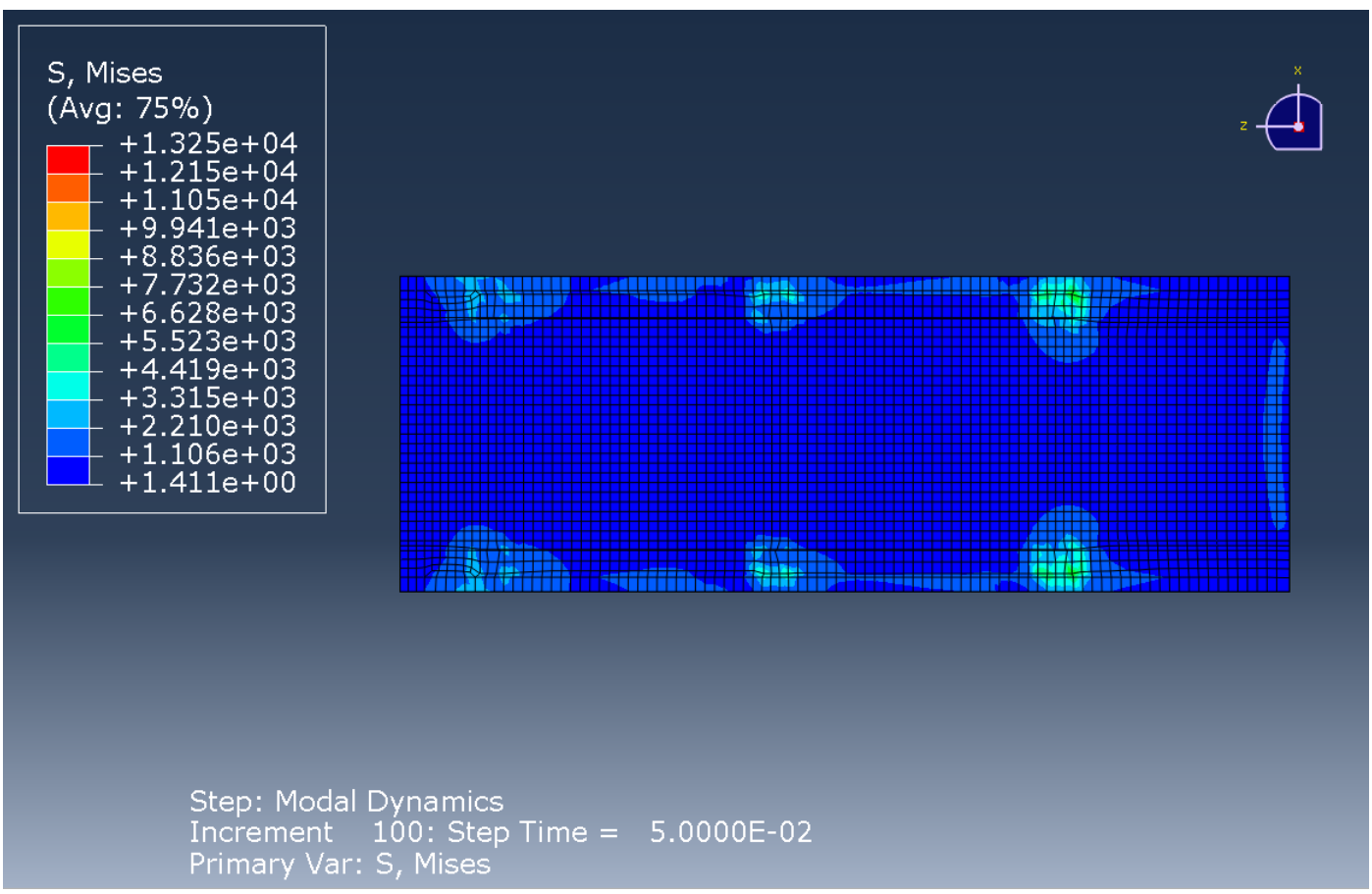

Figure 6.19: The screws closest to the applied load see the highest stress levels in the test pod with mass model FEA assembly. 
Using an overlay plot of the transient response for the empty and loaded test pod FEA assembly, it is clear each system has an equilibrium point of oscillation, but not necessarily at the same location (see Figure 6.20). The transient response of the loaded test pod also persists for a slightly longer period of time, compared to the empty test pod. Again, it can be assumed that the damping ratio $\zeta$ is less than one for the underdamped response of the empty and loaded test pod assemblies. From Figure 6.20, it is expected that the transient response of both assemblies would die off soon before reaching steady-state (see Appendix A.6 for more details).

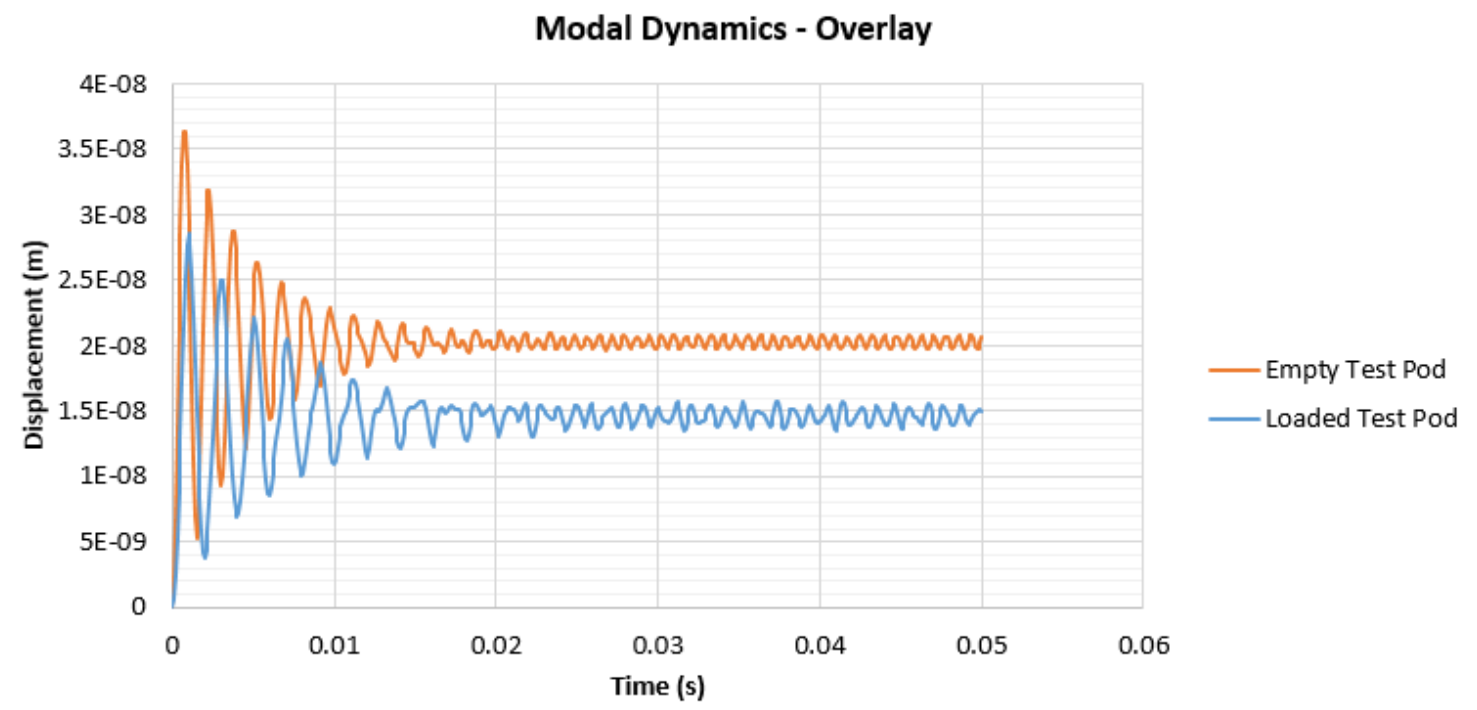

\section{Figure 6.20: Underdamped response overlay of the empty and loaded test pod FEA assembly.}

While structural failure is not expected for the test pod, mass model, and fixture plate under current CubeSat test standards, it is worth noting the stressed areas and how the assembly tends to deform under loading, as this can help with future equipment designs and setups. 


\subsubsection{Test Pod with CubeSat CPX}

Applying the same $1 \mathrm{~N}$ load, the stress distribution stays roughly the same as the empty and loaded test pod assembly containing the mass model (see Figure 6.21). This is expected as the force being applied to the assembly is relatively small.

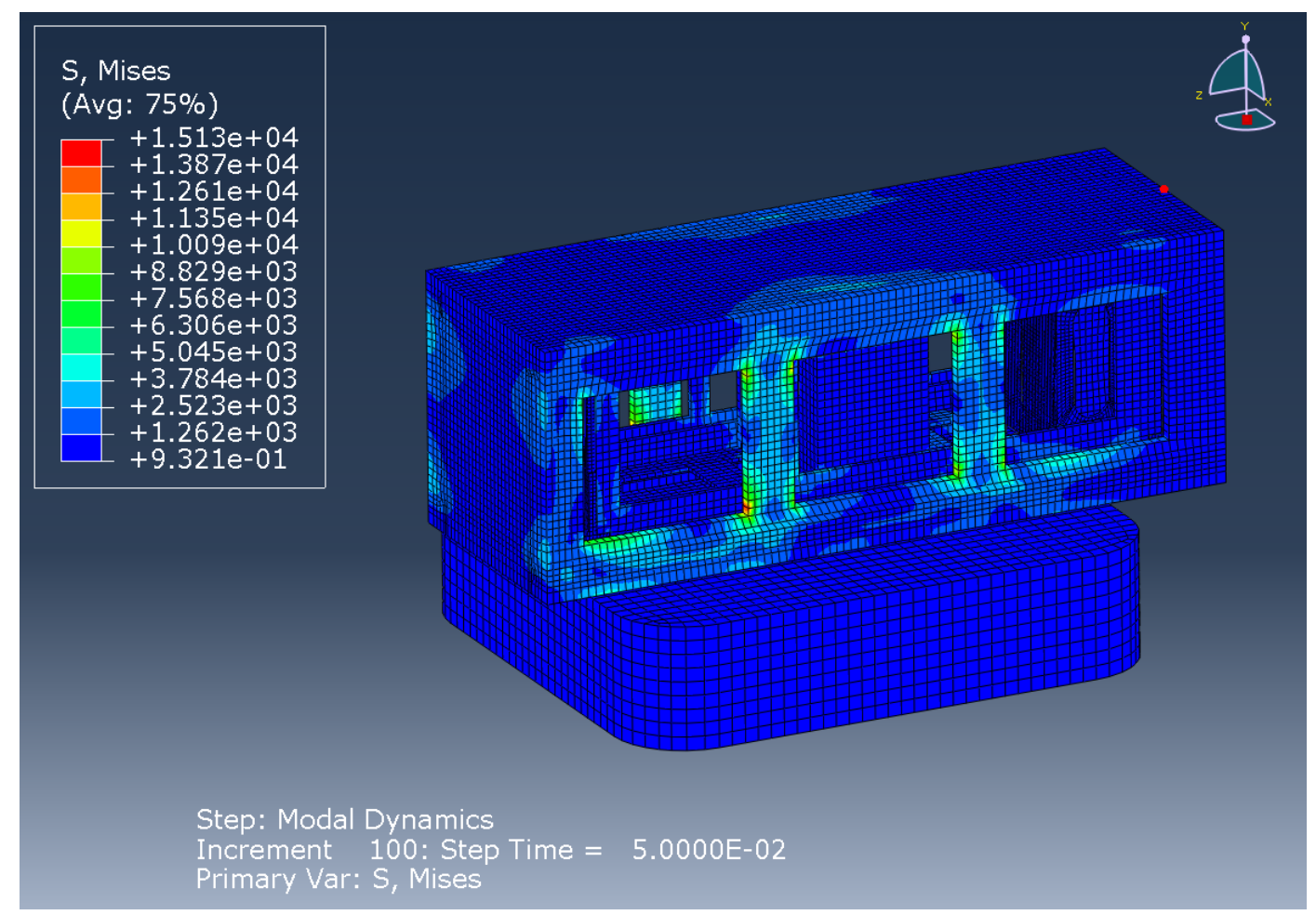

Figure 6.21: Stresses of the test pod and CPX assembly under a $1 \mathrm{~N}$ load.

The transient response of the CPX assembly exhibits an underdamped behavior (see Figure 6.22). The peak amplitudes of the test pod are less pronounced than CPX, due to the damping characteristics of the different structures and their constraints. The equilibrium point of oscillation between the test pod and CPX appear to have an offset, possibly due to the unbalanced mass distribution of the CPX structure. As expected, stresses around the screw areas are concentrated (see Figure 6.23), similar to the mass model FEA assembly. The CPX configuration with no payload has a similar damping behavior, so it is omitted to avoid redundancy. 


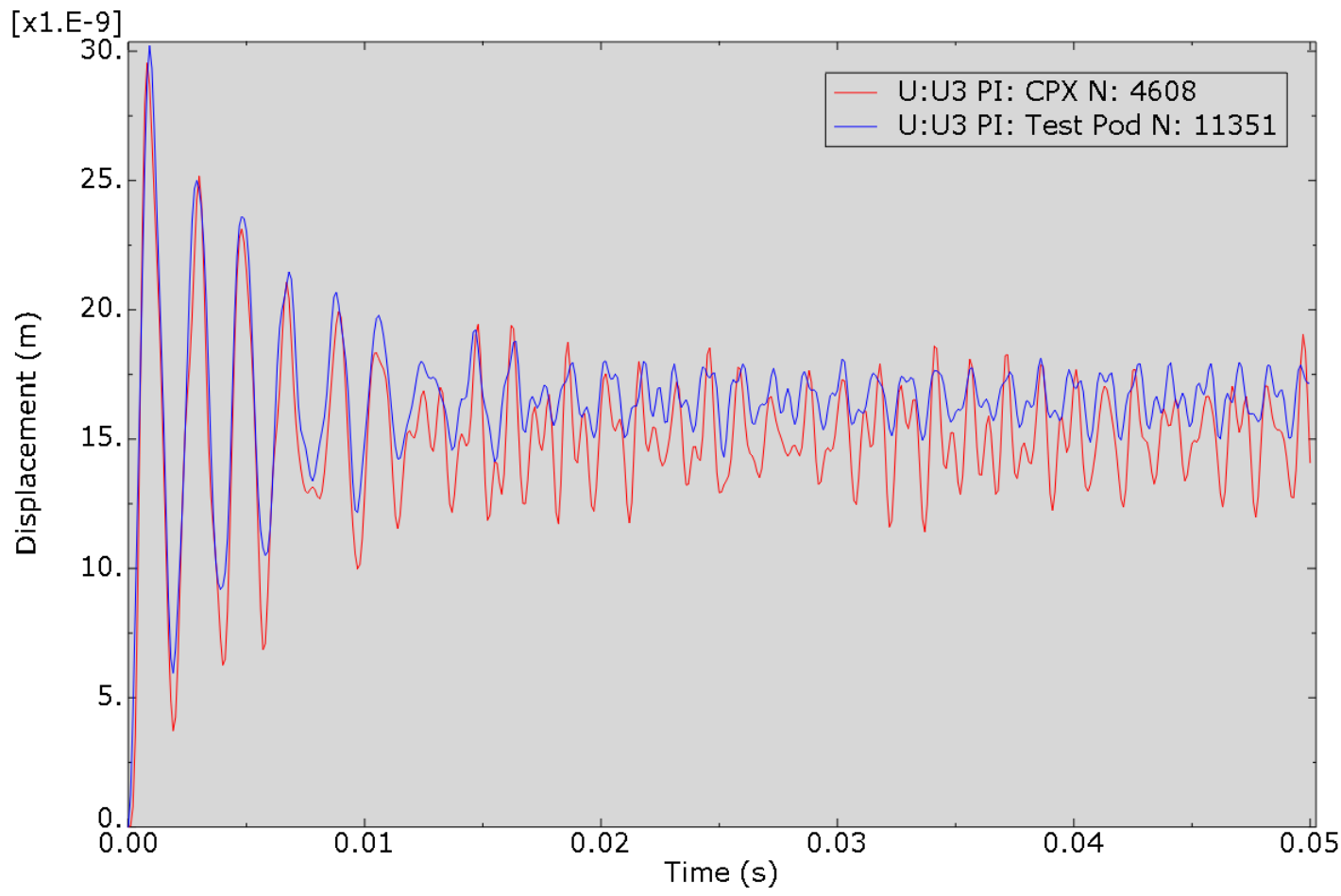

Figure 6.22: Transient response overlay of the test pod and CPX assembly.

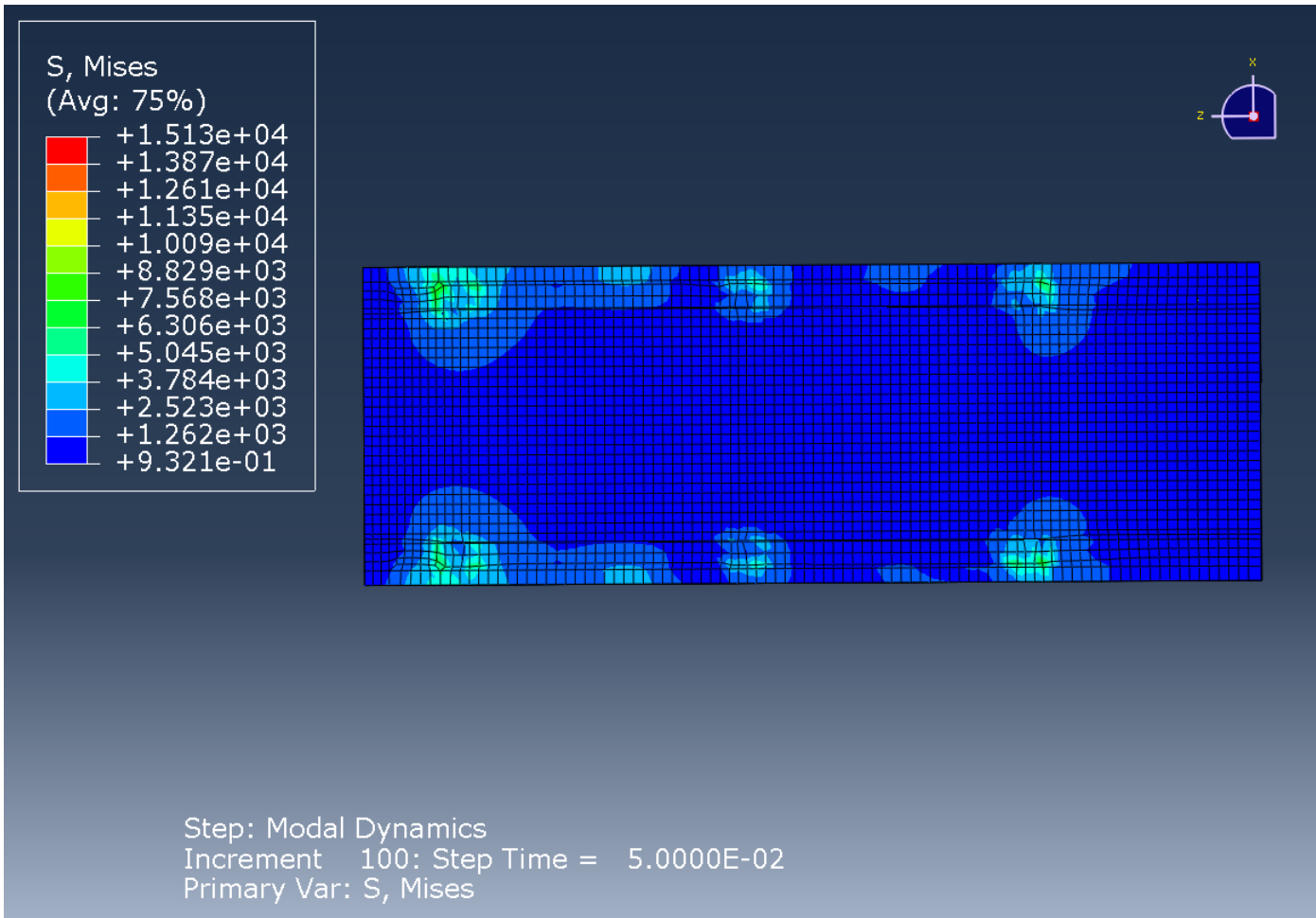

Figure 6.23: Stresses at the six screw locations of the test pod and CPX assembly. 


\subsection{Comparison of Experimental and FEA results}

\subsubsection{Test Pod}

Referring back to the sine sweep test data of the empty test pod assembly (Section 4.4.2.1), prominent peaks are now labeled to distinguish and identify the corresponding frequencies and amplitudes (see plot of Figure 6.24).

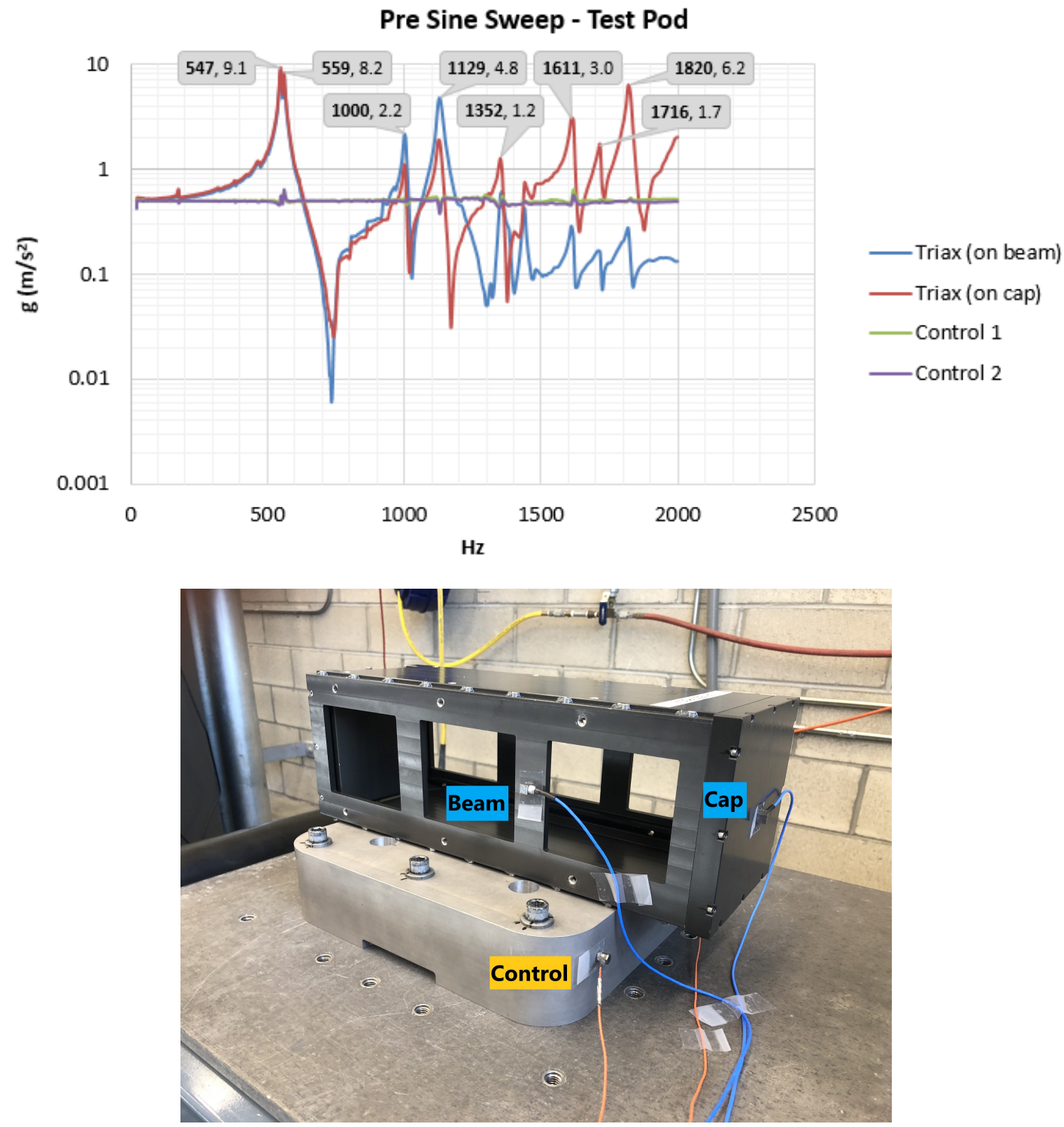

Figure 6.24: Notable peaks from the sine sweep empty test pod data. 
Taking the natural frequencies of the most prominent peaks and comparing to the values extracted from the frequency step in Abaqus (Section 6.1.1.1), the results show that the built FEA model approximates the first six natural frequencies of the test pod with an average error of $7 \%$ for the first six modes (see Table 6.2).

Table 6.2: Comparison of natural frequencies between experimental and FEA results for the empty test pod.

\begin{tabular}{|c|c|c|c|}
\hline Mode & Experimental $\mathbf{( H z )}$ & Abaqus $(\mathbf{H z})$ & Adams $(\mathbf{H z})$ \\
\hline 1 & 547 & 579 & 600 \\
\hline 2 & 559 & 665 & - \\
\hline 3 & 1000 & 1084 & - \\
\hline 4 & 1129 & 1192 & - \\
\hline 5 & 1352 & 1307 & - \\
\hline 6 & 1611 & 1620 & - \\
\hline 7 & 1716 & 2035 & - \\
\hline 8 & 1820 & - & \\
\hline
\end{tabular}

Recall that $1500 \mathrm{~Hz}$ is the rough threshold for a CubeSat vibration analysis due to the increased levels of noise that are present beyond this point when testing with current experimental equipment. So from these results, the FEA model successfully simulates the general natural frequencies of the empty test pod assembly.

Referring back to the random vibration test data of the empty test pod assembly (Section 4.4.2.2), prominent ASD peaks are also labeled to identify the corresponding frequencies and amplitudes with respect to the z-axis (see Figure 6.25). The FEA random response of Section 6.1.2.1 is also reprinted for comparison (see Figure 6.26). As previously mentioned, the response of the sine sweep and random vibration experimental data are correlated, and suggest that the empty test pod assembly has natural frequencies near $532 \mathrm{~Hz}$ and $1124 \mathrm{~Hz}$ when the assembly is excited in the Z-direction. 


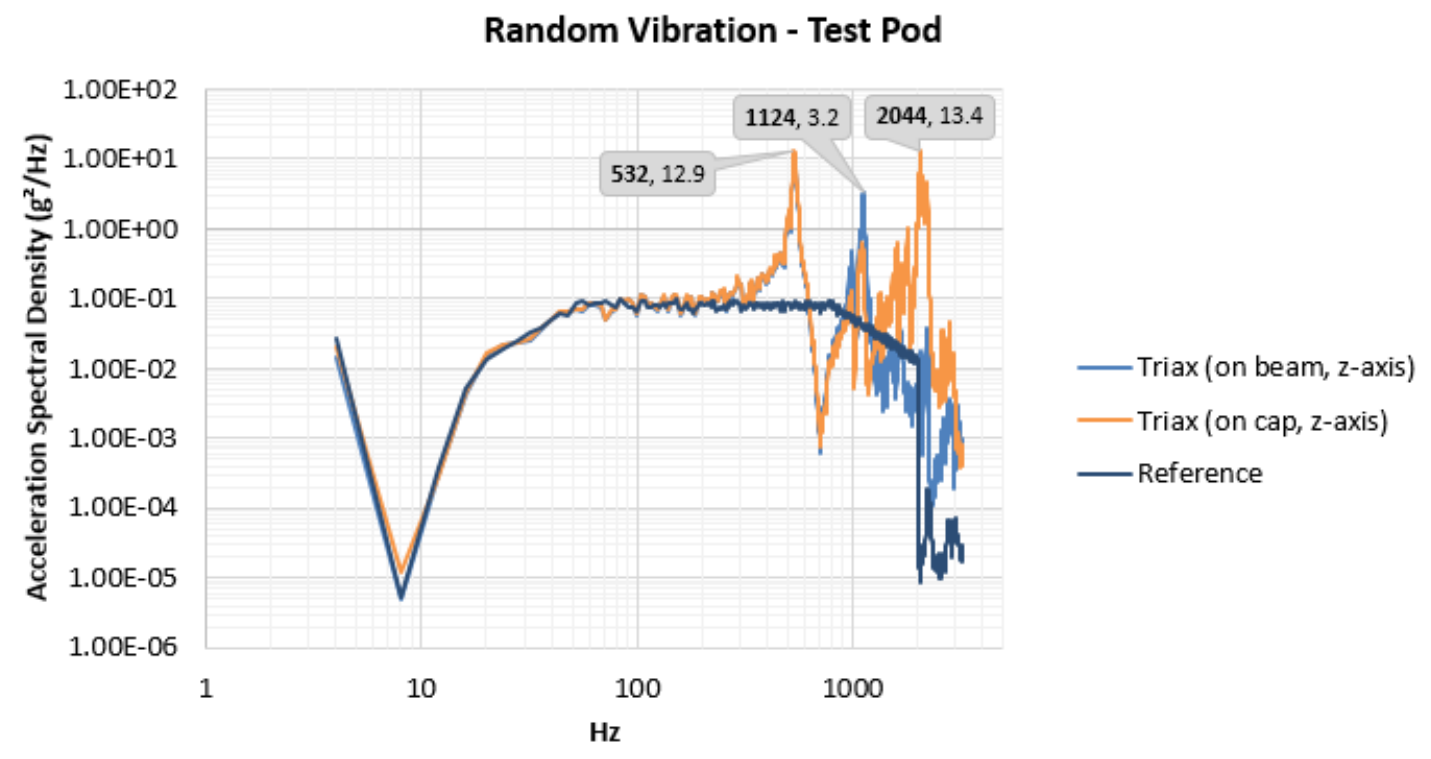

Figure 6.25: Prominent ASD peaks are labeled in the experimental data of the empty test pod.

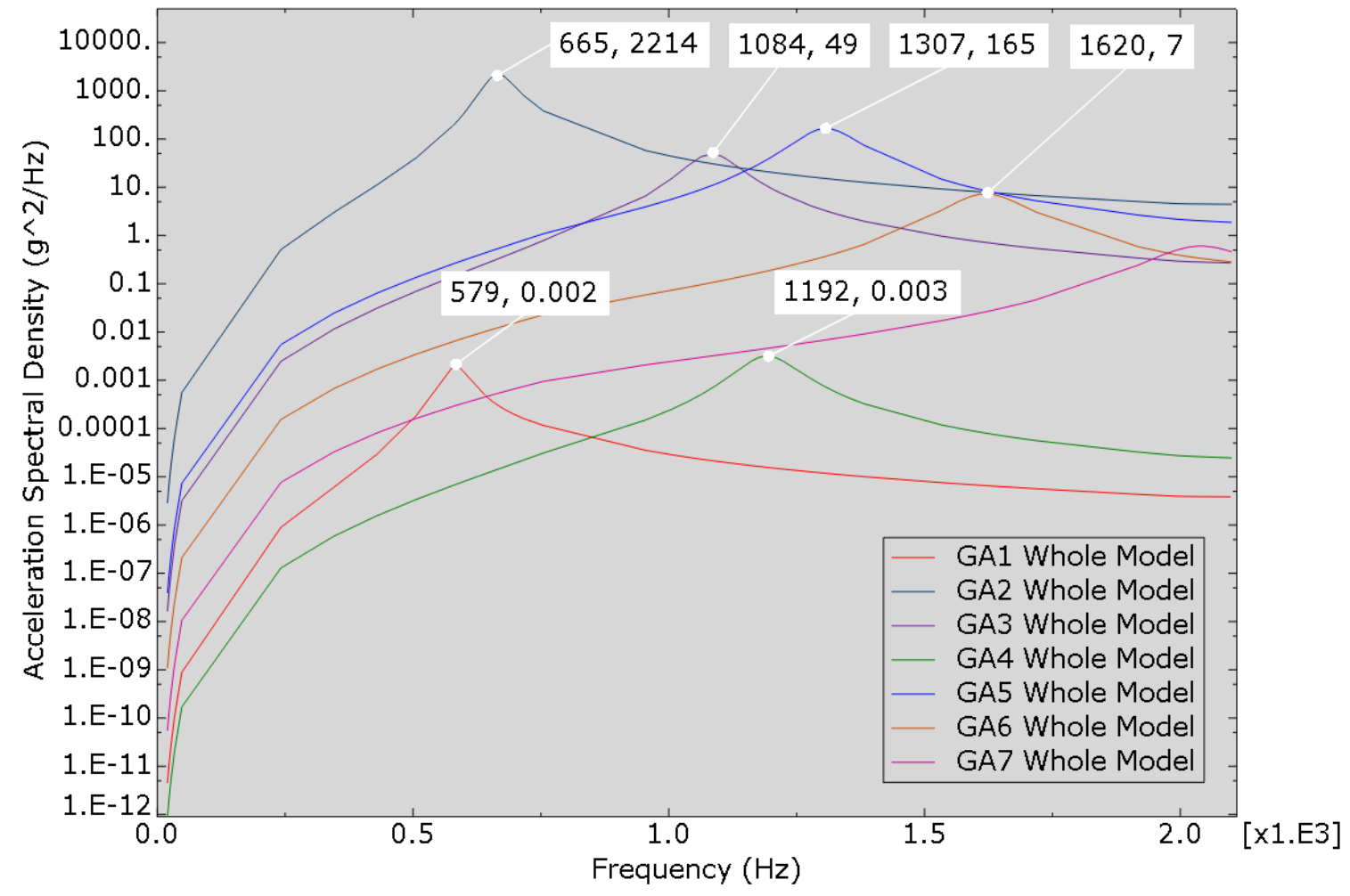

Figure 6.26: ASD peaks (first seven modes) of the FEA empty test pod model (reprinted for comparison). 
Taking the first two prominent ASD peaks of Figure 6.25, note that the FEA model predicts their corresponding frequencies with reasonable accuracy (see Table 6.3).

Table 6.3: Two prominent ASD peaks in the random vibration of the empty test pod.

\begin{tabular}{|c|c|c|}
\hline ASD Peak & Experimental $(\mathrm{Hz})$ & Abaqus $(\mathrm{Hz})$ \\
\hline 1 & 532 & 579 \\
\hline 2 & 1124 & 1192 \\
\hline
\end{tabular}

Although these two ASD peaks are not the most prominent in the FEA model (revert to Figure 6.26), factors like structural damping can be effecting the amplitudes, as this is a complex physical parameter that is difficult to replicate in FEA. A combination of other factors including the boundary conditions, mesh, and contact interactions could also be affecting the amplitudes of the FEA random response. Fine tuning the FEA model can help improve the accuracy in order to achieve amplitudes that are more in line with the experimental random vibration results. 


\subsubsection{Test Pod with Mass Model}

Referring back to the sine sweep test data of the loaded test pod assembly (Section 4.4.3.1), prominent peaks are now labeled to distinguish and identify the corresponding frequencies and amplitudes (see plot of Figure 6.27).

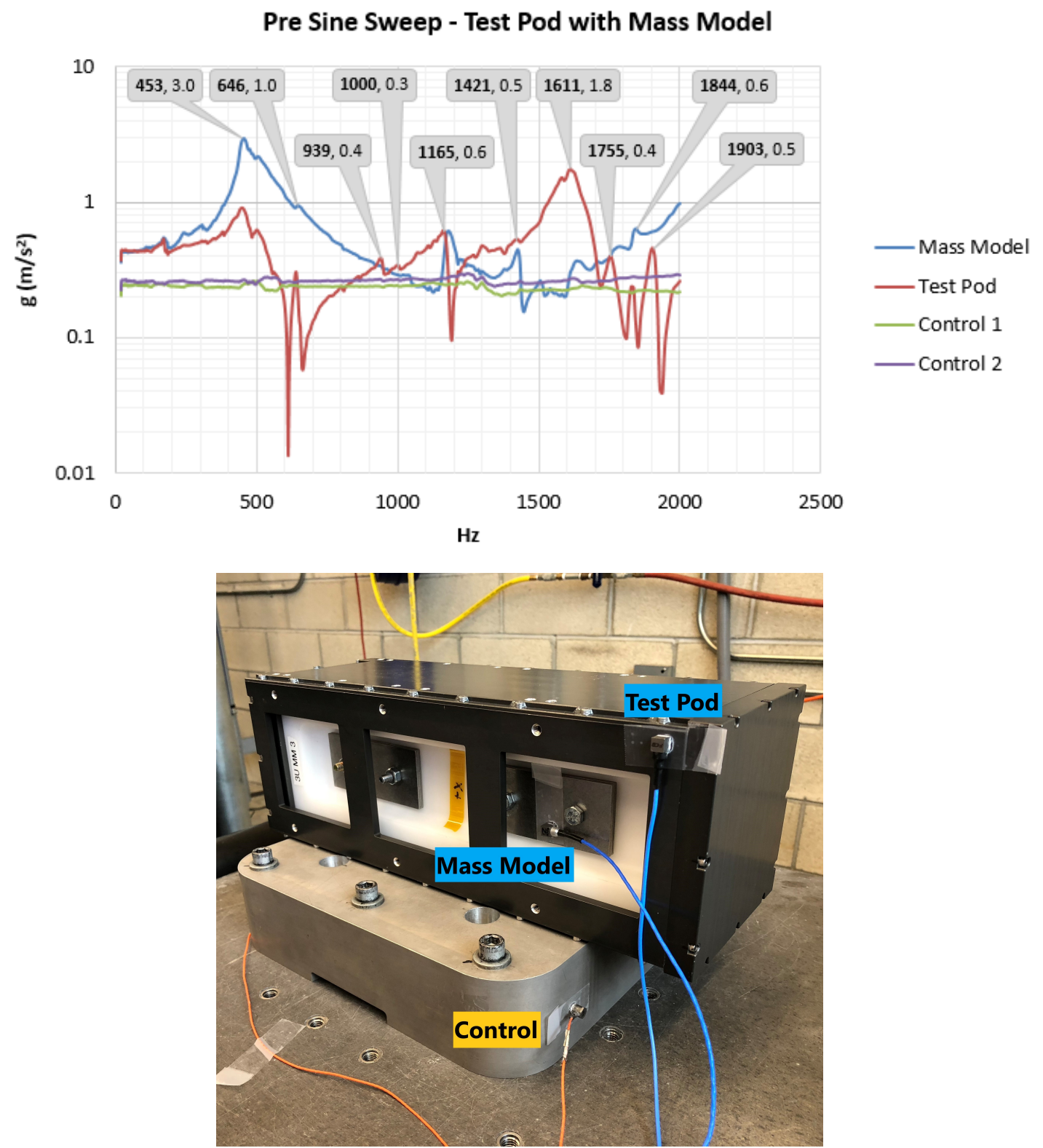

Figure 6.27: Notable peaks from the sine sweep loaded test pod data. 
Taking the natural frequencies of the most prominent peaks and comparing to the values extracted from Abaqus (Section 6.1.1.2), the results show that the built FEA model approximates the first eight natural frequencies of the loaded test pod with an average error of $4 \%$ for the first eight modes (see Table 6.4).

Table 6.4: Comparison of natural frequencies between experimental and FEA results for the loaded test pod.

\begin{tabular}{|c|c|c|}
\hline Mode & Experimental $(\mathbf{H z})$ & Abaqus $(\mathbf{H z})$ \\
\hline 1 & 453 & 497 \\
\hline 2 & 646 & 653 \\
\hline 3 & 939 & 964 \\
\hline 4 & 1000 & 1000 \\
\hline 5 & 1165 & 1281 \\
\hline 6 & 1421 & 1487 \\
\hline 7 & 1611 & 1711 \\
\hline 8 & 1755 & 1731 \\
\hline 9 & 1844 & 2024 \\
\hline 10 & 1903 & -- \\
\hline
\end{tabular}

Considering the $1500 \mathrm{~Hz}$ threshold due to noise, the results show that the FEA model successfully simulates the general natural frequencies of the loaded test pod assembly.

Referring back to the random vibration test data of the loaded test pod assembly (Section 4.4.3.2), prominent ASD peaks are also labeled to identify the corresponding frequencies and amplitudes with respect to the z-axis (see Figure 6.28). The FEA random response of Section 6.1.2.2 is also reprinted for comparison (see Figure 6.29). As previously mentioned, the response of the sine sweep and random vibration experimental data are correlated, but it should be noted that their first peak frequency differs by roughly $50 \mathrm{~Hz}$. Nonetheless, the data suggests that the loaded test pod assembly has natural frequencies near $400 \mathrm{~Hz}$ and $1100 \mathrm{~Hz}$ when the assembly is excited in the z-direction. 


\section{Random Vibration - Test Pod with Mass Model}

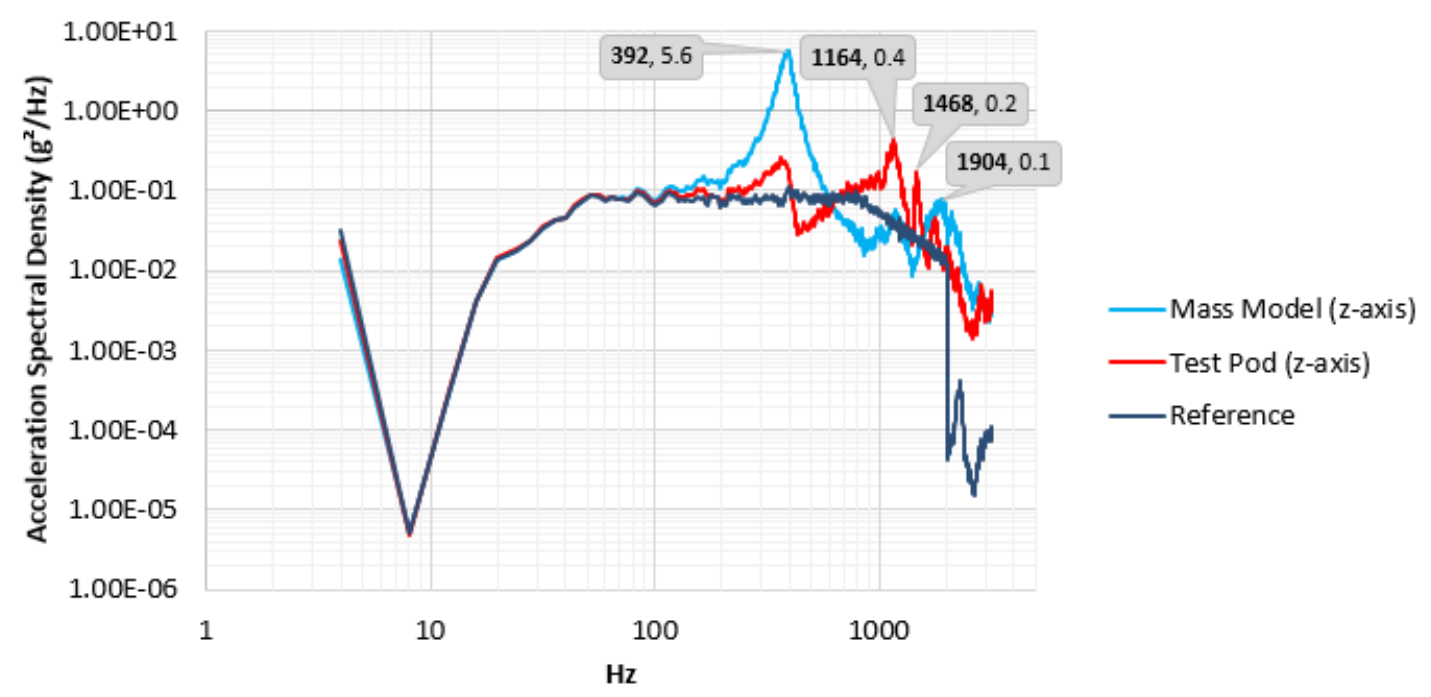

Figure 6.28: Prominent ASD peaks are labeled in the experimental data of the loaded test pod.

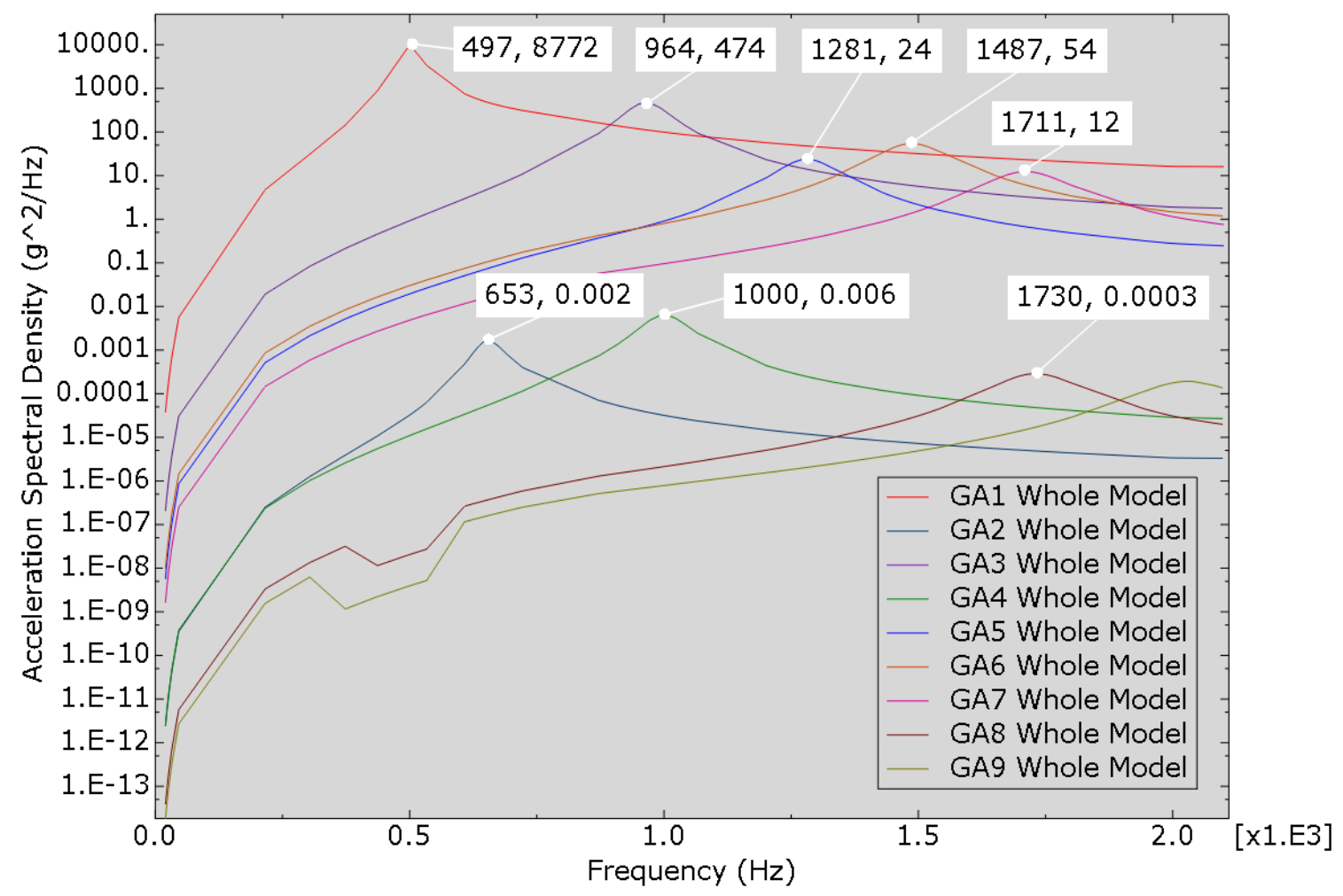

Figure 6.29: ASD peaks (first nine modes) of the FEA loaded test pod model (reprinted for comparison). 
Taking the first three prominent ASD peaks of Figure 6.28, note that the FEA model slightly overestimates their corresponding frequencies (see Table 6.5).

Table 6.5: Three prominent ASD peaks in the random vibration of the loaded test pod.

\begin{tabular}{|c|c|c|}
\hline ASD Peak & Experimental (Hz) & Abaqus (Hz) \\
\hline 1 & 392 & 497 \\
\hline 2 & 1164 & 1281 \\
\hline 3 & 1468 & 1487 \\
\hline
\end{tabular}

Although the frequencies of the three ASD peaks are slightly overestimated in the FEA model, their amplitude pattern is more in line with the experimental results (revert to Figure 6.29). Again, factors like damping and other system parameters can be tuned in the FEA model to improve the frequency prediction of the ASD peaks.

While tuning is recommended for the FEA model, multiple sets of experimental data should also be collected using different test runs. This would help reduce the variance in the data in order to more accurately identify the true natural frequencies of the test pod and mass model assembly. These frequencies can then be used as a concrete baseline for optimizing the FEA models.

Although the geometries of the test pod, mass model, and fixture plate were simplified for the FEA analysis, the simple bodies still prove to be useful in the dynamic analysis of the test pod assembly. While constraints and boundary conditions are more complex in the test pod and vibration table assembly (e.g. contact pressures, friction, cross vibrations, etc.), a simplified FEA model can still yield relatively accurate results that are representative of the real system. 


\subsubsection{Test Pod with CubeSat CPX}

Since CubeSat CPX is a concept design, there is no experimental data to use for a direct comparison. However, as the CPX structure shares some geometric similarities with the Cal Poly PolySat structure discussed in Section 3.3 (see Figure 6.30), vibration test results for this structure were pulled from the CubeSat lab data bank. The experimental data of the PolySat structure was used to identify possible correlations that may exist between the two structures if CPX were to be manufactured and tested.
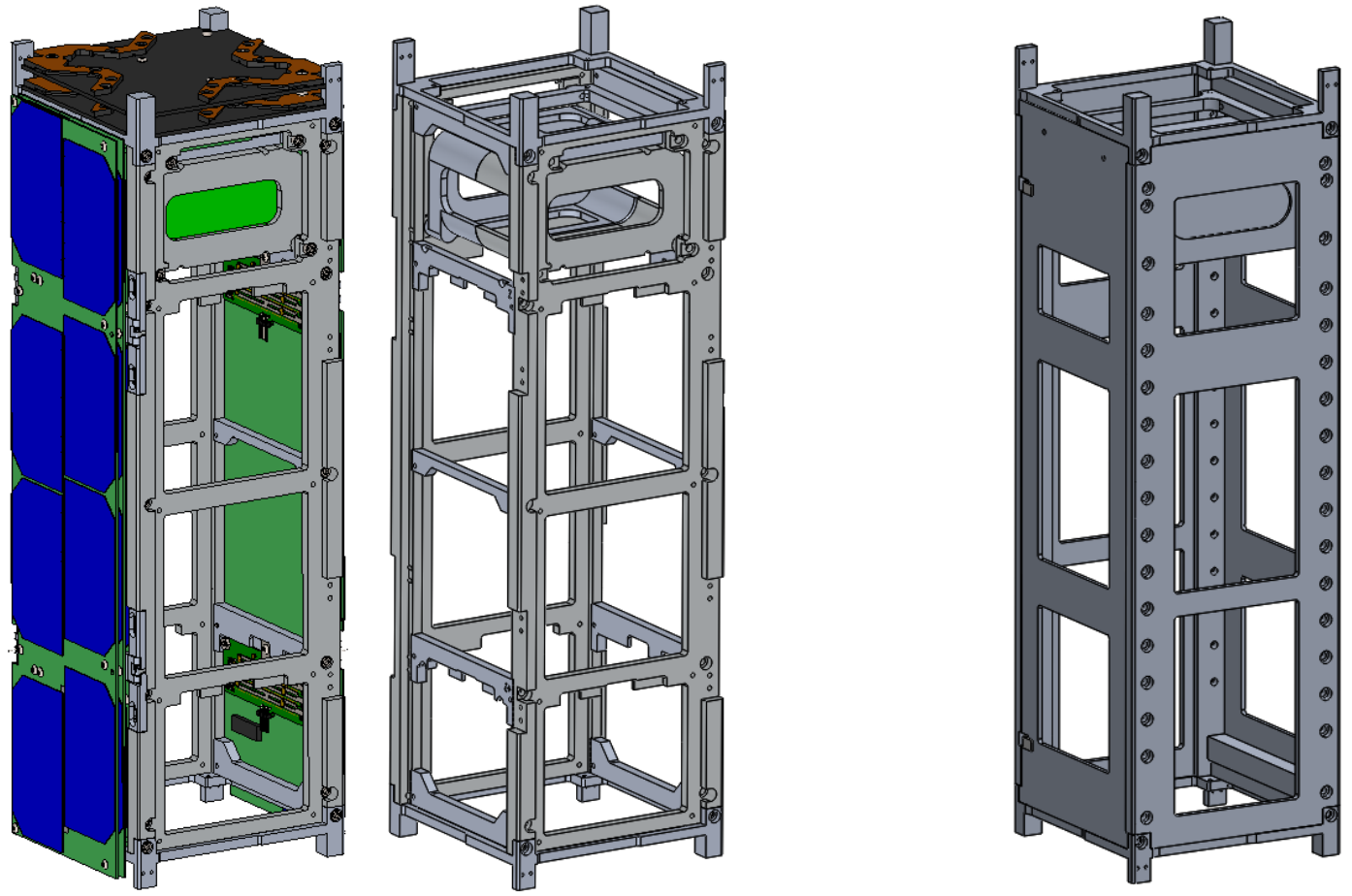

Figure 6.30: PolySat structure ${ }^{23}$ (left) and CubeSat CPX (right). 
From the sine sweep test data (z-axis) of the PolySat structure (see Figure 6.31), note that the two most prominent natural frequencies in the response occur within $550 \mathrm{~Hz}$, with the largest peak amplitude at approximately $350 \mathrm{~Hz}$.

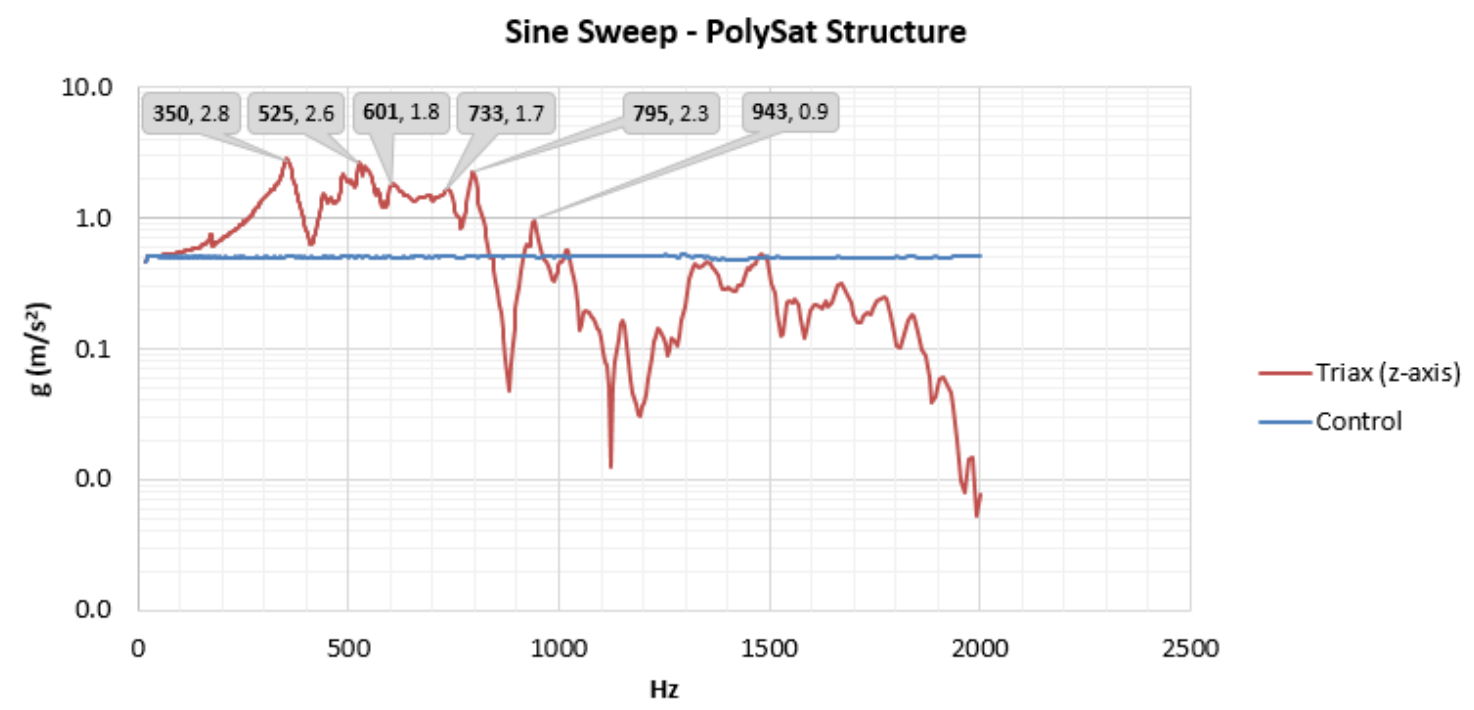

Figure 6.31: Notable peaks in the sine sweep data of the PolySat structure.

Comparing these values to the natural frequencies extracted from the CPX FEA model (Section 6.1.1.3), the two structures share similar natural frequencies up to approximately $800 \mathrm{~Hz}$ (see Table 6.6). Recall that not including a payload in the FEA CPX structure significantly increases the number of natural frequencies, in part due to the unsupported thin panels of the structure midsection.

Table 6.6: Natural frequencies of the PolySat and CPX structures.

\begin{tabular}{|c|c|c|c|}
\hline \multirow{2}{*}{ Mode } & Experimental (Hz) & \multicolumn{2}{|c|}{ Abaqus (Hz) } \\
\cline { 2 - 4 } & PolySat Structure & CPX & CPX (no payload) \\
\hline 1 & 350 & 357 & 153 \\
\hline 2 & 525 & 517 & 153 \\
\hline 3 & 601 & 604 & 276 \\
\hline 4 & 733 & 714 & 276 \\
\hline 5 & 795 & 835 & 330 \\
\hline 6 & 943 & 838 & 376 \\
\hline
\end{tabular}


From the random vibration test data of the PolySat structure (see Figure 6.32), note that the most prominent ASD peak (with respect to the z-axis) occurs at approximately $360 \mathrm{~Hz}$. These results correlate with the sine sweep data test data of Figure 6.31, which is supporting evidence that the PolySat structure has a natural frequency at approximately $360 \mathrm{~Hz}$ when excited in the z-direction.

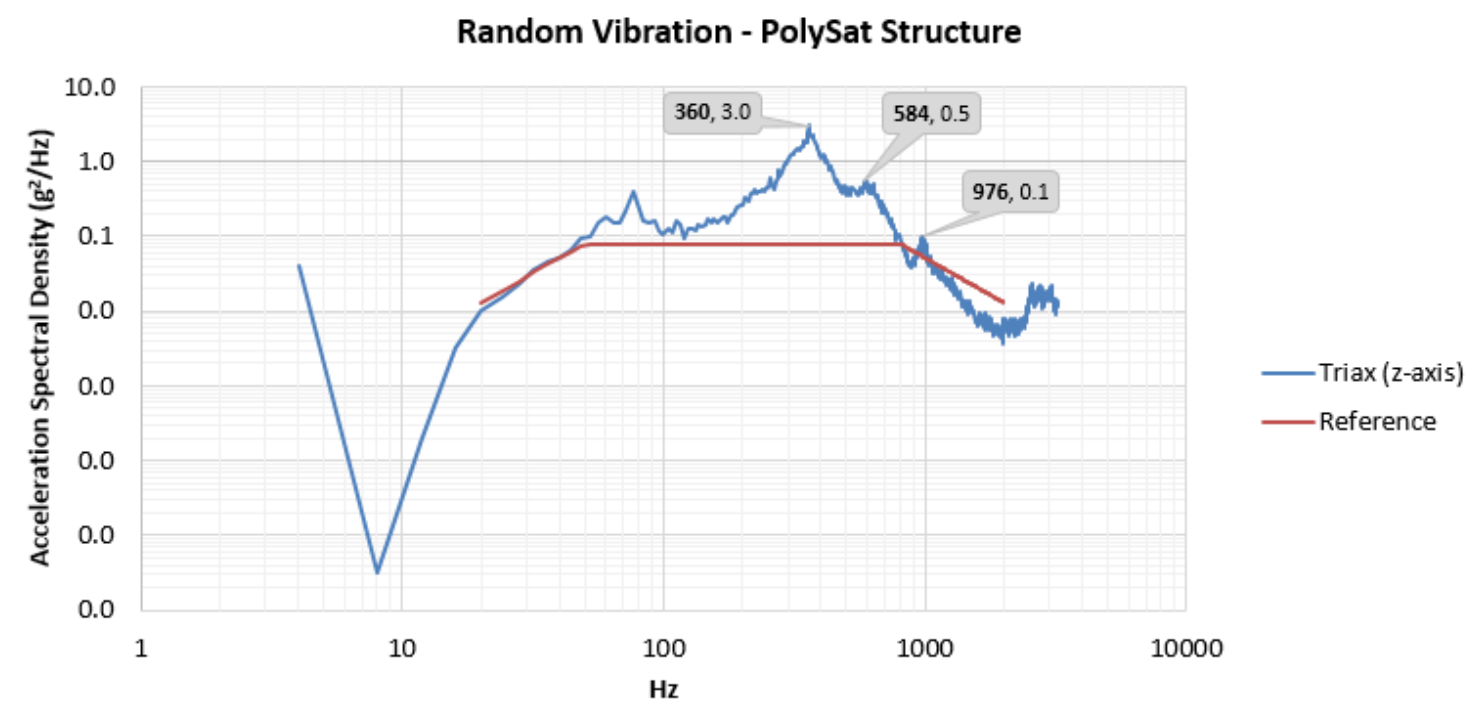

Figure 6.32: Notable peaks in the random vibration data of the PolySat structure.

Comparing the random vibration data of the PolySat structure to the random response of the CPX structure of Section 6.1.2.3 (see Figure 6.33), note that the two structures share an ASD peak frequency near $360 \mathrm{~Hz}$ (z-axis), but their amplitudes at this frequency are quite different. Again, this may be tied to the accuracy of the system parameters in the FEA model. Three prominent ASD peaks for the two structures are noted in Table 6.7 for comparison. 


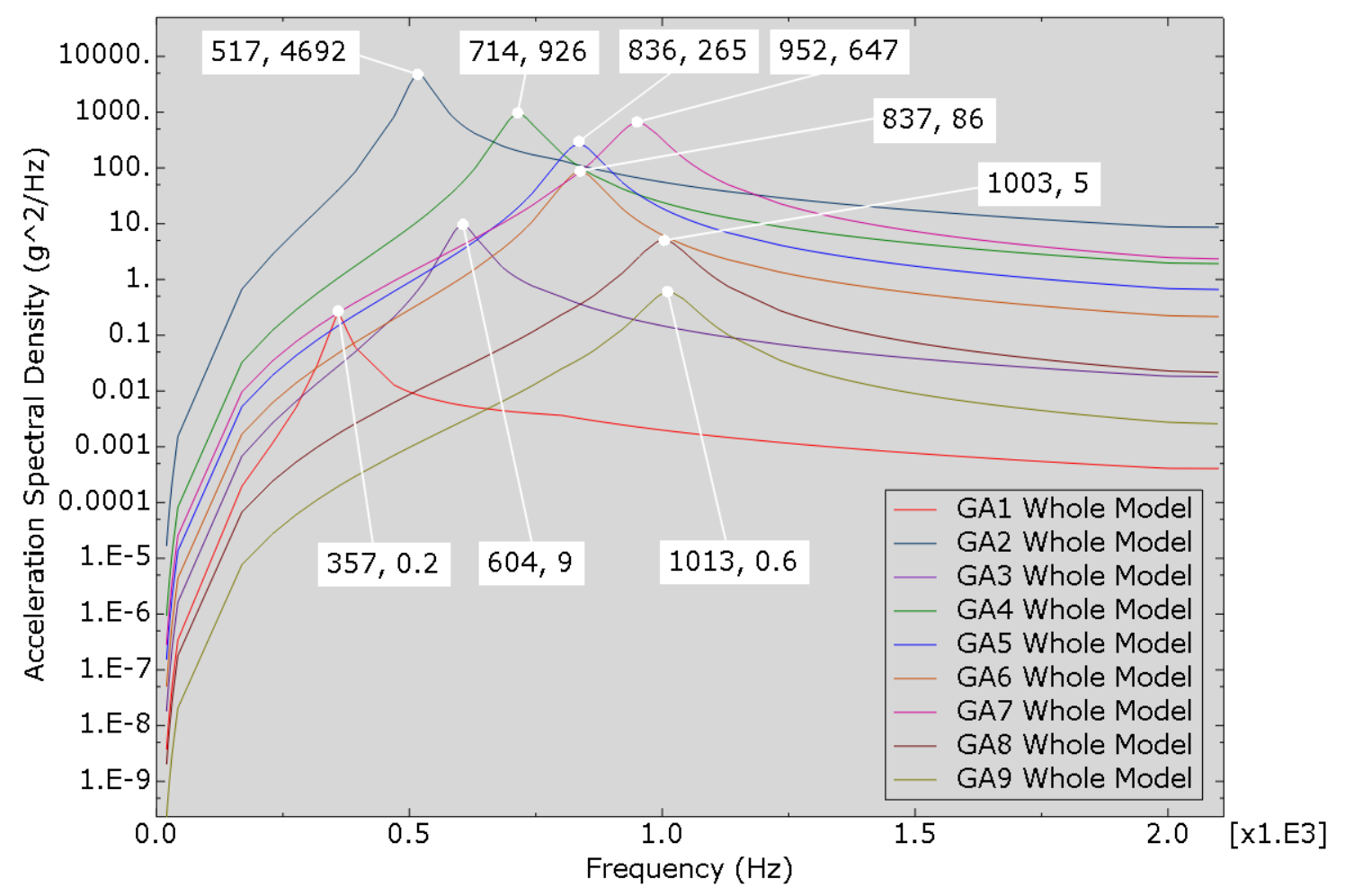

Figure 6.33: ASD peaks (first nine modes) of the test pod with CPX FEA assembly (reprinted for comparison).

Table 6.7: Prominent ASD peaks of the PolySat and CPX structures.

\begin{tabular}{|c|c|c|c|}
\hline \multirow{2}{*}{ Peak } & Experimental (Hz) & \multicolumn{2}{|c|}{ Abaqus (Hz) } \\
\cline { 2 - 4 } & PolySat Structure & CPX & CPX (no payload) \\
\hline 1 & 360 & 357 & 376 \\
\hline 2 & 584 & 604 & 614 \\
\hline 3 & 976 & 1003 & 1019 \\
\hline
\end{tabular}

So from the FEA frequency and random response, the evidence suggests that although the CPX and PolySat structures are different in design, enough similarities exist that the CPX response falls within reasonable range of what would be expected in vibration test data of such a structure. The results presented in this chapter are meant to serve as an example to show that the FEA models can help predict the dynamic behavior of a CubeSat assembly prior to manufacturing and integration. 


\section{Chapter 7}

\section{CONCLUSION}

\subsection{Summary}

CubeSats withstand a wide range of dynamic loads during the launch phase, and must undergo rigorous testing to qualify for launch vehicle integration. It is important to understand the dynamic behavior of CubeSat assemblies to ensure the structure and subsystems will remain intact and functional upon release into outer orbit. So in this work, an FEA dynamic analysis was performed on a pseudo P-Pod (test pod), pseudo CubeSat (mass model), fixture plate, and concept structure (CPX) in order to generate the natural frequencies, mode shapes, and damping behavior observed in vibration test data. Focus was placed on the z-axis of the test pod, which runs along the longitudinal direction of the structure. Simplified CAD models were created in SolidWorks to represent the four bodies, and were then imported to Abaqus, which is an FEA program that can be used to simulate the dynamic response of a system. The CAD models were meshed and assembled using a set of constraints, interactions, and boundary conditions that resemble those of the real system. With the assemblies completed, vibration test parameters were set using the frequency, random response, and modal dynamic steps in Abaqus. Upon running the frequency analysis, the first 6 natural frequencies were generated for the empty test pod assembly, with an average $7 \%$ error when compared to experimental data. Mode $2(665 \mathrm{~Hz})$ had largest influence on displacement along the z-axis of the empty test pod assembly. Similarly, the analysis was repeated for the test pod and mass model assembly, and the first 8 natural frequencies were generated, with an average $4 \%$ error when compared to 
experimental data. Unlike the empty test pod assembly, Mode $1(497 \mathrm{~Hz})$ had the largest influence on displacement along the z-axis of the loaded test pod assembly. After running the modal dynamic analysis with an applied instantaneous load, both assemblies exhibited a response that was in line with that of an underdamped system, which coincides with the response observed in the experimental data.

The same analysis was performed for the CPX design (see Figure 7.1). Since CPX is a concept structure, no direct experimental data was available. Instead, the CPX response was compared to data of a similar structure designed by PolySat (Cal Poly SLO). The first five natural frequencies of CPX were similar to the PolySat structure up to approximately $800 \mathrm{~Hz}$. The PolySat and CPX structures had a prominent natural frequency near $360 \mathrm{~Hz}$ when excited in the z-direction. From the modal dynamic analysis, the CPX assembly exhibited an underdamped response, which coincides with the response observed in general CubeSat vibration test data.
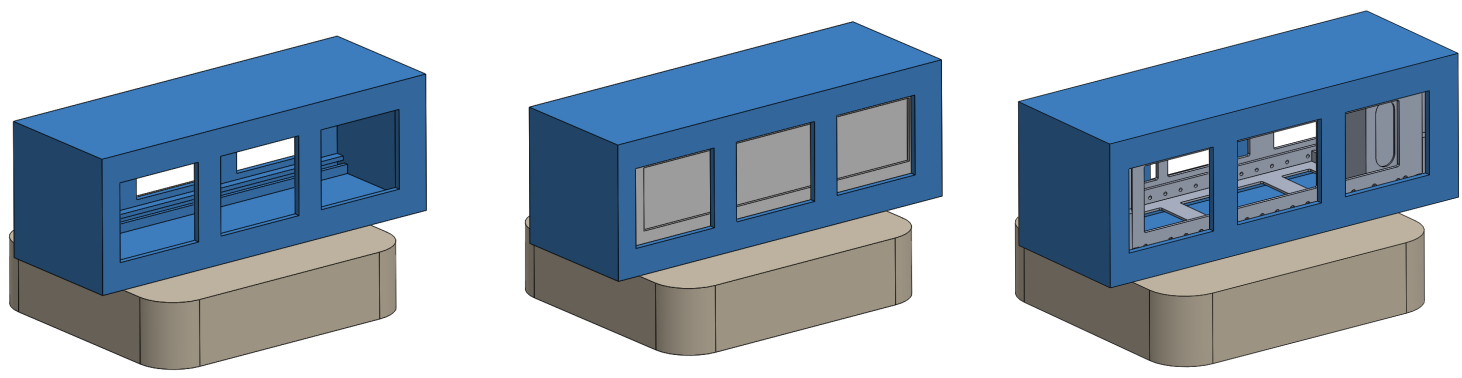

Figure 7.1: Test pod (left), mass model (center), and CPX assemblies (right) evaluated in this work. 


\subsection{Learnings}

Using an FEA approach to approximate the dynamic behavior of a system can be of great benefit when designing the components of such a system. Despite the complexity that an assembly might present in the system, a simplified FEA model can still yield results that approximate the real system response. Constraints and boundary conditions can have significant effects on the response, and while they can be simplified in the FEA model, they should still be representative of the real system. The dynamic behavior of a system is not limited to the response of a particular or dominating component, but is instead a combination of the component responses and the interactions between them.

While FEA can be performed on specific components of a CubeSat for structural analysis (see Figure 7.2), these results do not always reflect true launch conditions. The CubeSat structure, P-Pod, and launch vehicle all introduce additional factors in the response that are not present in the sole analysis of a component. While it is difficult to create a model that incorporates all the dynamic factors of a launch, future work can continue to build upon the FEA models presented here to achieve results that approach the true dynamic response of a CubeSat.

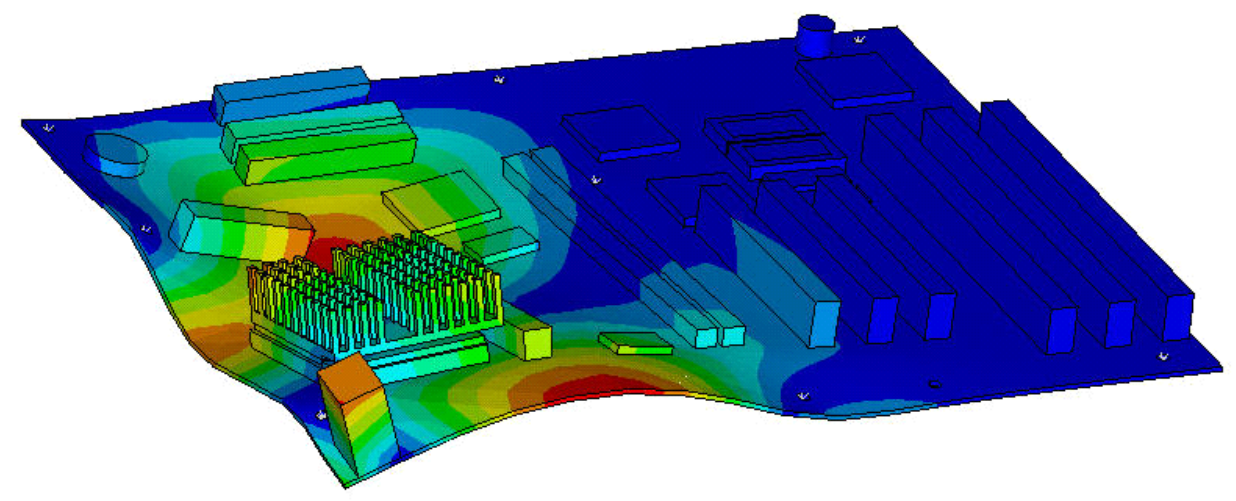

Figure 7.2: CubeSat circuit boards under vibration loads are susceptible to bending and losing components due to poor soldering. ${ }^{63}$ 


\subsection{Notes and Recommendations}

The fixture plate used for testing and FEA modeling of the test pod assemblies allowed for overhang due to its small size. From the studies of Section 6.1.1.1, recall that the fixture plate influences the natural frequencies of the test pod. So to achieve more accurate results from future vibration tests and FEA models, it is recommended that a wider fixture be used (see Figure 7.3). This will remove the overhangs, add stability to the assembly, and provide uniform support throughout the entire bottom panel of the test pod.

The placement of accelerometers during vibration tests should be kept consistent throughout vibration testing. It is recommended that markings and/or photos be used to keep track of the accelerometer attachment points throughout all tests. Cross vibrations are an inherent side effect of vibration tables, so using consistent setups will help minimize the unknowns that are affecting the accuracy of the system response.

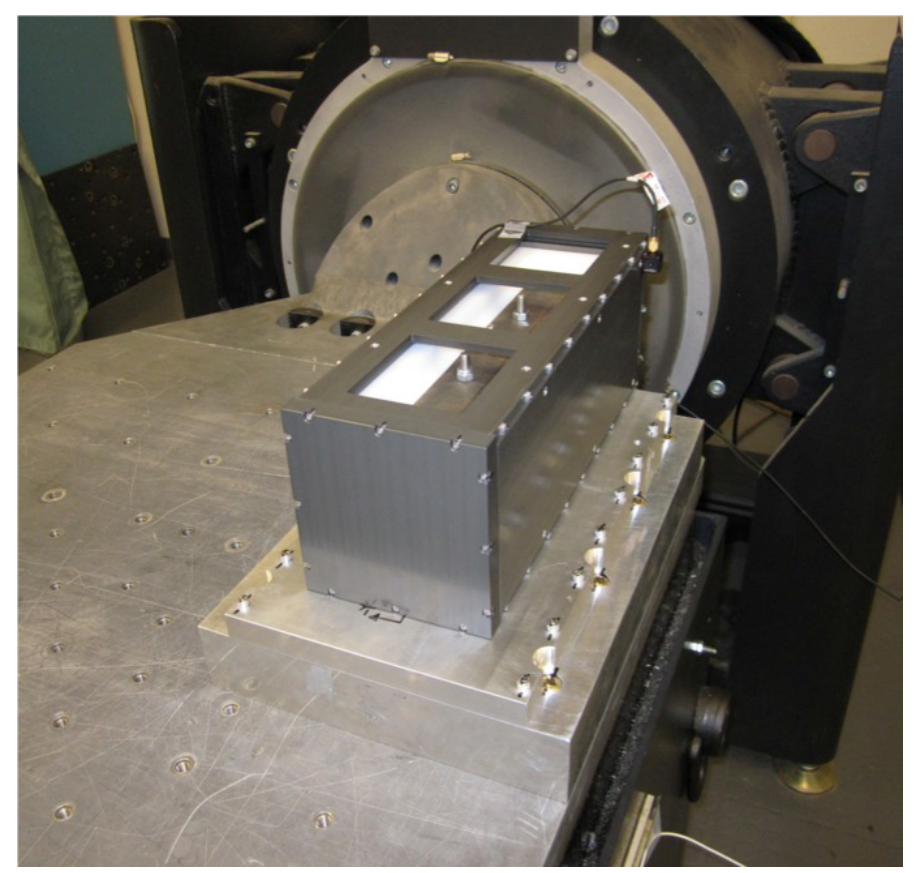

Figure 7.3: Optional fixture plate that can be used with the test pod. Note that the test pod base is fully supported by this fixture plate. ${ }^{22}$ 


\subsection{Next Steps}

While this analysis was limited to using pseudo structures and a concept structure design, the results of the test pod, mass model, and CPX are still representative of a P-Pod and CubeSat assembly. Furthermore, the FEA analysis can be extended and improved by using the CAD models of the P-Pod and the CubeSat (see Figure 7.4) being considered for a mission. While the P-Pod is considered a legacy (proven) structure, including it in FEA models can help identify design and assembly flaws of a CubeSat structure that may become apparent after manufacturing, assembly, and testing have been completed.

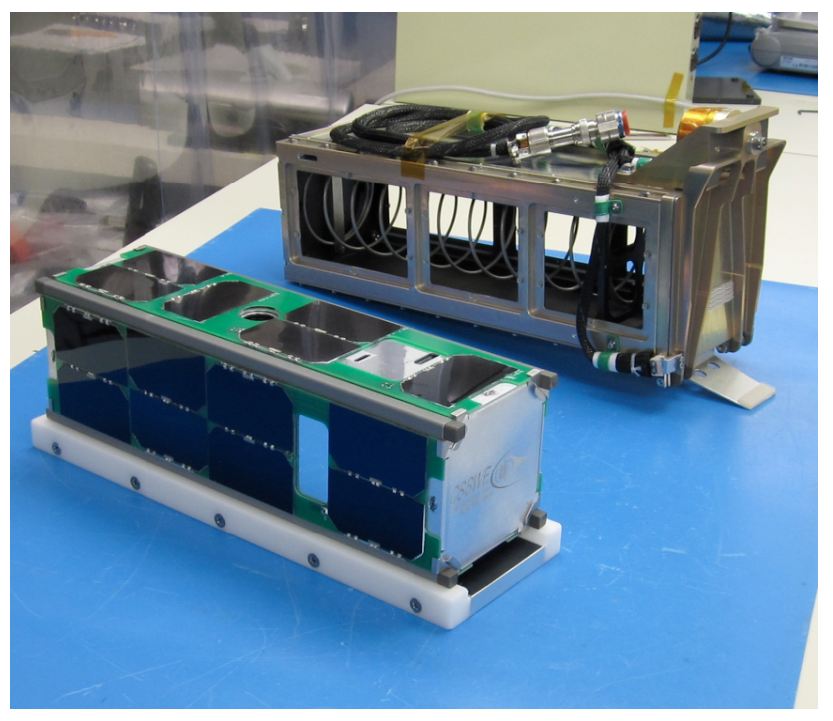

Figure 7.4: CubeSat and P-Pod prior to integration. ${ }^{27}$

With sufficient validation, the CPX concept can be manufactured and tested, so a direct comparison can be made to the FEA model presented in this work. A prototype of CPX can also help determine if the proposed features of the design are feasible in the long run. Catching flaws early in the design phase of a CubeSat will help save time and cost during development, and lead to an optimized design and assembly that can withstand the rigors of a space launch. 


\section{BIBLIOGRAPHY}

[1] 3d stress components.

http://academic. uprm. edu/pcaceres/Courses/MMII/IMoM-5A.pdf.

[2] 3u cubesat. https://grabcad.com/library/3u-cubesat.

[3] 3u-cubesat. https://grabcad.com/library/3u-cubesat.

[4] 5 again. http://www.winchurchill2.com/stories/2018/03/28/5-again/.

[5] 75 years after infamous tacoma narrows bridge collapse: Not so fast! https://www.txstate.edu/news/news_releases/news_archive/2015/ November-2015/TacomaNarrows110315.html.

[6] Abaqus. https://en.wikipedia.org/wiki/Abaqus, journal=Wikipedia.

[7] Abaqus analysis user's guide (6.14). https://www.sharcnet.ca/Software/Abaqus/6.14.2/v6.14/books/usb/ default.htm?startat=pt06ch28s01alm01.html.

[8] Abaqus analysis user's manual (6.10). https://www. sharcnet.ca/Software/Abaqus610/Documentation/docs/ v6.10/books/usb/default.htm?startat=pt06ch24s01abo23.html.

[9] Abaqus analysis user's manual (6.10). https://www. sharcnet.ca/Software/Abaqus610/Documentation/docs/ v6.10/books/usb/default.htm?startat=pt06ch26s06abo25.html.

[10] abaqus analysis user's manual (6.10). https://www.sharcnet.ca/Software/Abaqus610/Documentation/docs/ v6.10/books/usb/default.htm?startat=pt03ch06s03at12.html. 
[11] Abaqus/CAE user's manual (6.10). https://www. sharcnet.ca/Software/Abaqus610/Documentation/docs/ v6.10/books/usi/default.htm?startat=pt03ch17s05s01.html.

[12] About ELaNa. https://www.nasa.gov/content/about-elana, journal $=$ NASA, publisher $=$ NASA.

[13] About NASA General Environmental Verification Specification. https: //ntrs.nasa.gov/archive/nasa/casi.ntrs.nasa.gov/20030106019.

[14] About NASA's Goddard Space Flight Center. https://www.nasa.gov/centers/goddard/about/index.html.

[15] Accelerometers. https://www.pc-control.co.uk/accelerometers.htm.

[16] Accelerometers. http://www.industrial-electronics.com/DAQ/ industrial_electronics/input_devices_sensors_transducers_ transmitters_measurement/Accelerometers.html.

[17] Advanced nonlinear analysis - linear vs. non-linear analysis in AutoPIPE. https://communities.bentley.com/products/pipe_stress_analysis/ w/pipe_stress_analysis__wiki/21892/f-advanced-nonlinearanalysis-linear-vs-non-linear-analysis-in-autopipe.

[18] An analytical solution to free rectangular plate natural vibrations by beam modes ordinary and missing plate modes. https://www.researchgate.net/publication/310744242_An_ Analytical_Solution_to_Free_Rectangular_Plate_Natural_ Vibrations_by_Beam_Modes__-_Ordinary_and_Missing_Plate_Modes.

[19] Artist concept of a Cubesat in space. 
https : //Www . americaspace.com/2012/02/02/nasa-seeks-proposalsfor-edison-small-satellite-demonstrations/cubesat-full/.

[20] Back to elements - tetrahedra vs. hexahedra.

https://support.ansys.com/staticassets/ANSYS/staticassets/ resourcelibrary/confpaper/2004-Int-ANSYS-Conf-9.PDF.

[21] Beam analysis validation. https://mechanicalc.com/calculators/beam-analysis/validation.

[22] Cal Poly SLO - CubeSat. http://www. cubesat.org/.

[23] Cal Poly SLO - PolySat. http://www.polysat.org/.

[24] Chapter 8 linear strain triangle (overview). https : //www.slideserve.com/bertha/chapter-8-linear-straintriangle-overview.

[25] Chladni figures (speaker driven) (c 38). http: //tsgphysics.mit . edu/front/?page=demo $\cdot$ php\&letnum=C38, journal=TSG@MIT Physics.

[26] Coefficient of friction. http: //www.engineershandbook.com/Tables/frictioncoefficients.htm.

[27] Colorado student space weather experiment. https://en.wikipedia.org/ wiki/Colorado_Student_Space_Weather_Experiment.

[28] Comparison of the mechanical behaviour between stiff and flexible adhesive joints for the automotive industry. https://www.researchgate.net/publication/248922757_Comparison_ of_the_Mechanical_Behaviour_Between_Stiff_and_Flexible_ Adhesive_Joints_for_the_Automotive_Industry. 
[29] Continuum solid shell elements. https://abaqus-docs.mit.edu/2017/ English/SIMACAEELMRefMap/simaelm-c-solidcont.htm.

[30] Cubesat challenge - random vibration analysis. https://www . youtube. com/watch?v=BLpWBsG_NHM.

[31] damped shm. https://pedronfigueiredo.com/damped-shm/.

[32] Determine the jacobian matrix [j] and its determinant for the elements shown in figure p1011.... https://www.transtutors .com/questions/determinethe-jacobian-matrix-j-and-its-determinant-for-the-elementsshown-in-figure-1980186.htm.

[33] Developing a safer way to machine magnesium. https://www.mmsonline.com/articles/developing-a-safer-way-tomachine-magnesium.

[34] Dynamic Response Optimization. http://www . eurostag. be/en/products/eurostag/functions/dynamicresponse/dynamic-response/.

[35] Fem for frames (finite element method) part 1.

$$
\begin{aligned}
& \text { http://what-when-how.com/the-finite-element-method/fem-for- } \\
& \text { frames-finite-element-method-part-1/. }
\end{aligned}
$$

[36] Figure found online, source unknown, credit given to rightful owner of content.

[37] Forced vibration of a cantilever beam (continuous system). http://vlab. amrita. edu/?sub=3\&brch=175\&sim=1081\&cnt=1.

[38] GRIFEX Technology Validation Cubesat Launched with SMAP. https://esto.nasa.gov/news/news_GRIFEXlaunch_2_2015.html. 
[39] GSA to play a major role in galileo satellite launch planned for dec 12. https://www.gsa. europa.eu/newsroom/news/gsa-play-major-rolegalileo-satellite-launch-planned-dec-12.

[40] GSFC-STD-7000 (pdf). https://standards.nasa.gov/standard/gsfc/gsfc-std-7000.

[41] How to write a conclusion for an essay: Guide for beginners. https://justbuyessay.com/blog/essay-conclusion.

[42] Hydraulic shock absorber. http://www.hitachi-automotive.co.jp/en/ products/shock/products/function_structure/index.html.

[43] Interference beats and tartini tones. http://www . animations.physics . unsw. edu.au/jw/beats. htm.

[44] Introduction: System modeling. http://ctms . engin .umich.edu/CTMS/index. php?example=Introductionion=SystemModeling.

[45] Introduction to finite element method/finite element analysis (fem/fea). https://www.mechlectures. com/introduction-to-finite-elementmethodfinite-element-analysis-femfea/.

[46] An investigation of resonance. http://www.math.lsa.umich.edu/ glarose/ classes/diffeq/0ld2/resonance.html.

[47] Laser doppler vibrometer. https://en.wikipedia.org/wiki/Laser_Doppler_vibrometer.

[48] Launch Environment (pdf). http://www.nasa.gov/.

[49] Matlab for all steps of dynamic vibration test of structures. https://www . intechopen.com/books/applications-from- 
engineering-with-matlab-concepts/matlab-for-all-steps-ofdynamic-vibration-test-of-structures.

[50] Mechanical engineering - Theory \& Practice of rotor dynamics. https://nptel.ac.in/courses/112103024/23.

[51] Mechanical vibration Chapter 2: Free vibration of undamped 1-dof linear system. http://www . posterus. sk/?p=14142.

[52] Model 356a03 triaxial icp accelerometer installation and operating manual (pdf). http: //www . pcb.com/products . aspx?m=356a03.

[53] Msc software corporation-adams. https://www.mscsoftware.com/product/adams.

[54] New metamaterial could improve sound wave technologies. https://www.rdmag.com/article/2019/01/new-sensor-could-helpdiagnose-developmental-disabilities-children-0.

[55] On the eigenfrequencies of a cantilever beam with attached tip mass and a spring-mass system. https://www.researchgate.net/publication/ 256801296_On_the_eigenfrequencies_of_a_cantilever_beam_with_ attached_tip_mass_and_a_spring-mass_system.

[56] Overview in the application of fem in mining and the study of case: Stress analysis in pulleys of stacker-reclaimers: Fem vs. analytical. https://www . intechopen. com/books/finite-element-analysisapplications-in-mechanical-engineering/overview-in-theapplication-of-fem-in-mining-and-the-study-of-case-stressanalysis-in-pulleys-of-sta. 
[57] Parameterizing the dimensions of imported cad files. https://www.comsol.jp/blogs/parameterizing-dimensionsimported-cad-files/.

[58] PCB impact hammer for vibration test. https : //www . edaboard. com/showthread.php?152373-PCB-ImpactHammer-for-Vibration-Test.

[59] Poisson's ratio. https://www.engineeringtoolbox.com/poissons-ratio-d_1224.html.

[60] Random vibration-an overview. http://www. emtengineering.com/wpcontent/uploads/2013/04/Barry-Controls-Random-Vibration .pdf.

[61] Second-order spring mass damper: Measuring poles. http://web.mit.edu/hchin/Public/activlab/lab3.html.

[62] Shear locking. http://mokka.ibmdatamanagement.co/shear-locking/.

[63] Shock and vibration finite element analysis of a printed circuit board. https://www . mechsol. com/case-study/shock-and-vibration-finiteelement-analysis-of-a-printed-circuit-board/.

[64] Simple harmonic motion. https://minireference.com/physics/simple_harmonic_motion.

[65] Solid mechanics: what are the differences between plane stress and plane strain conditions? https://www.quora.com/Solid-Mechanics-What-are-thedifferences-between-plane-stress-and-plane-strain-conditions.

[66] Spring plunger (8476a36). https://www.mcmaster.com/8476a36. 
[67] Stochastic simulation and lagrangian dynamics. http://stochasticandlagrangian.blogspot.com/2011/07/what-doesshape-function-mean-in-finite.html.

[68] Structural dynamics and the seismic response control laboratory. http://people.duke.edu/ hpgavin/.

[69] Swarm communications and cubesats: An update on the advantages and challenges of nanosatellites. https://www . allaboutcircuits. com/news/swarm-communicationcubesats-advantages-challenges-nanosatellites-satellite/.

[70] Tesseract cubesat bus with deployable solar panels. https://digitalcommons . calpoly.edu/cgi/viewcontent.cgi?referer=https://www.google.com/ \&httpsredir=1\&article=1013\&context=bkendowments.

[71] Transformation of stress. https://www . engineering.com/Library/ArticlesPage/tabid/85/ ArticleID/149/Transformation-of-Stress . aspx.

[72] Two approaches to measuring acceleration. https://www . sensorsmag. com/components/two-approaches-tomeasuring-acceleration.

[73] Types of oscillations - damped oscillation and undamped oscillation. http://www . circuitstoday.com/types-of-oscillations.

[74] UKube-1. https://earth.esa.int/web/eoportal/satellitemissions/u/ukube-1.

[75] Underdamped second-order system. https://dademuchconnection. wordpress . com/2017/11/14/underdamped-second-order-system/. 
[76] Understanding nonlinear analysis. https://www.solidworks.com/sw/docs/ Nonlinear_Analysis_2010_ENG_FINAL.pdf.

[77] Unholtz-Dickie Corp. website. https://www.udco.com/products/sliptables/st-slip-table-assemblies/.

[78] User:egm6936.s09/curling of paper sheet using shell theory. https://en.wikiversity.org/wiki/User: Egm6936.s09/Curling_of_paper_sheet_using_shell_theory.

[79] A very brief look at driven harmonic motion. http://spiff.rit.edu/ classes/phys312/workshops/w5c/driven_theory.html.

[80] Vibration for the machine design materials pe exam. https://www . engproguides. com/machine-vibration.html.

[81] What is a hammer test or hammer impact test? http: //blog.prosig.com/2013/02/11/what-is-hammer-impact-testing/.

[82] What is shear locking in fem? https://caendkoelsch. wordpress . com/2018/09/21/what-is-shearlocking-in-fem/.

[83] The world's ten longest continuous truss bridges.

https://www. enr.com/articles/38496-the-worlds-ten-longestcontinuous-truss-bridges?v=preview.

[84] D. J. Inman. Engineering vibration. Pearson, 2014.

[85] D. L. Logan. A first course in the finite element method. Cengage Learning, 2012. 


\title{
APPENDICES
}

\author{
Appendix A
}

\section{VIBRATION THEORY}

\section{A.1 Free Vibration}

The field of vibrations is the culmination of various disciplines within engineering including dynamics, strength of materials, and the application of mathematical differential equations. ${ }^{84}$ By applying these principals and the known parameters of a particle (e.g. mass, spring constant), a set of equations can be derived that help describe the motion and behavior of the particle. These equations of motion (EOM) help define the displacement, velocity, and acceleration of the particle, and as such, can even be used to define more complex systems that involve more than one particle at at time.

The spring-mass model is a classic example of vibration where a mass is attached to a spring and allowed to oscillate under its own weight in free vibration (see Figure A.1). By developing a free body diagram (FBD) of the model, it is possible to equate the forces acting on the mass (weight due to gravity) and the restoring force of the spring. 


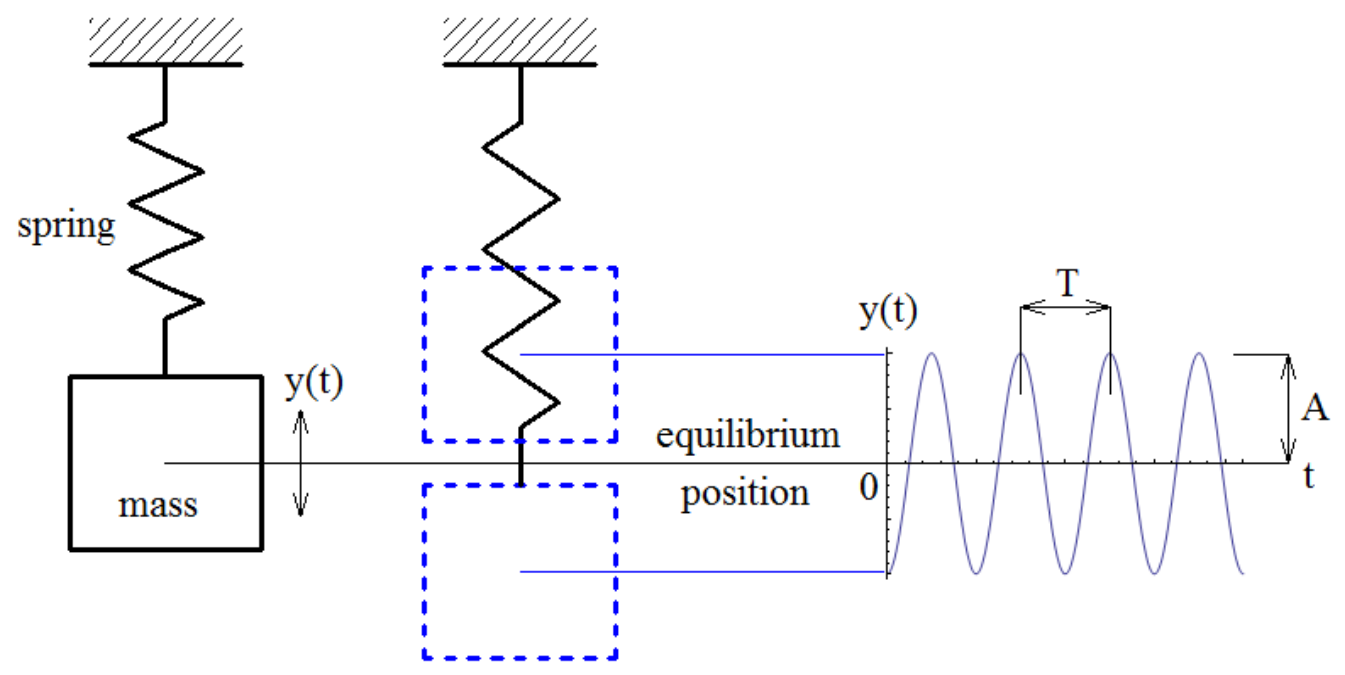

Figure A.1: Free vibration of an undamped 1-DOF linear system. ${ }^{51}$

The restoring force $f_{k}$ of a spring can be defined as

$$
f_{k}=k x
$$

where $k$ represents the stiffness of the spring over a displacement $x$. This is a linear relationship as long as the spring is not stretched beyond its limit of plastic deformation.

If the spring constant $k$ and mass $m$ are known for a system, then the frequency of oscillation can be determined. This is known as the natural frequency of the system, or $\omega_{n}$, and is defined as

$$
\omega_{n}=\sqrt{\frac{k}{m}}
$$

Using a slightly modified spring-mass model (see Figure A.2), 


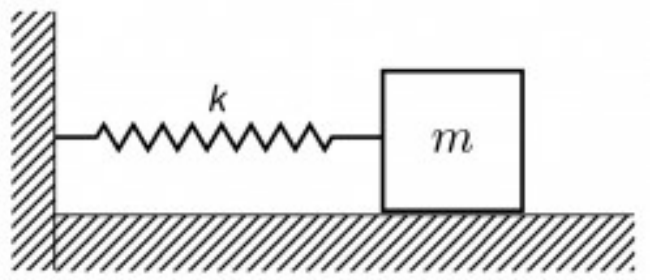

Figure A.2: Spring-mass system with negligible friction. ${ }^{64}$

the forces from the free body diagram of this model can be summed over the $\mathrm{x}-$ direction (assume horizontal) to yield

$$
m \ddot{x}(t)+k x(t)=0
$$

where $\ddot{x}$ represents acceleration as the second derivative of the displacement $x$. Similarly, velocity can also be derived using the first derivative of the displacement.

Once determined, the natural frequency can be used to calculate the period $\mathrm{T}$ of the model

$$
T=\frac{2 \pi}{\omega_{n}}
$$

where $T$ is the time it takes for a cycle to repeat itself (revert to Figure A.1). Furthermore, by applying initial conditions for displacement $x_{0}$ and velocity $v_{0}$, the amplitude, or peak height $A$ (revert to Figure A.1) can be determined by

$$
A=\frac{1}{\omega_{n}} \sqrt{\omega_{n}^{2} x_{0}^{2}+v_{0}^{2}} \text {, where } \quad v_{0}=\text { initial velocity }
$$

The phase $\phi$ represents the initial value of the repeating sine function, and combining with the other system parameters, the solution to the model becomes

$$
x(t)=\frac{1}{\omega_{n}} \sqrt{\omega_{n}^{2} x_{0}^{2}+v_{0}^{2}} \sin \left(\omega_{n} t+\phi\right), \quad \text { where } \phi=\tan ^{-1}\left(\frac{\omega_{n} x_{0}}{v_{0}}\right)
$$

where $\mathrm{x}(\mathrm{t})$ is the free response of the spring-mass model. The oscillatory motion of such a model is known as simple harmonic motion. 


\section{A.2 Damping}

While a system in free vibration may oscillate indefinitely, such models are not as common in nature due to the inherent effects of damping. One type of damping is known as viscous damping, and refers to the resistance of motion due to a viscous element (e.g. oil, water, air) that dissipates energy away from a moving object. ${ }^{84}$ As a result, viscous damping has the effect of decaying the motion of a system in free vibration, ultimately bringing the system to rest as the motion dies out (see Figure A.3).

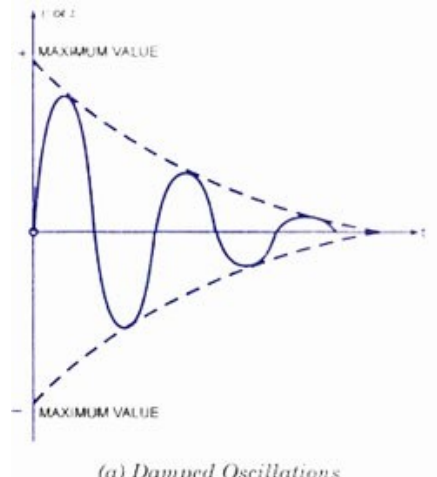

(a) Damped Oscillations

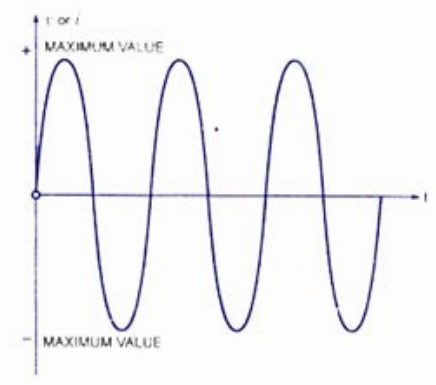

(b) Undamped or Sustained Oscillations

Figure A.3: Damped (left) vs Undamped (right) system. $^{73}$

Dashpots for example use the principal of viscous damping, including the more commonly known shock absorber which is used in vehicle suspension systems and heavy machinery (see Figure A.4). In this device, the piston valve has perforations that allow oil to flow from one side of the valve to the other, and so dissipating energy away from the sliding piston rod. This is the function of shock absorbers as a vehicle hits a bump on the road, where the sudden jolt or "shock" is damped by the flow of oil, and a high-pressured gas restores the piston rod to its neutral position. 


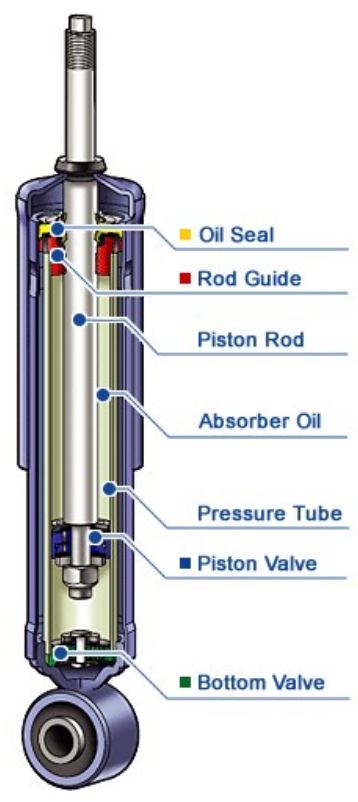

Figure A.4: Shock absorber functions as a viscous damper. ${ }^{42}$

Just like the spring force $f_{k}$ is proportional to displacement, the damping force $f_{c}$ is proportional to the velocity of the moving rod and defined as

$$
f_{c}=c \dot{x}(t)
$$

where $c$ is the damping coefficient related to the viscous element, and $\dot{x}(t)$ representing velocity as the first derivative of the displacement $x$.

Using a modified spring-mass system that now includes damping $c$ (see Figure A.5),

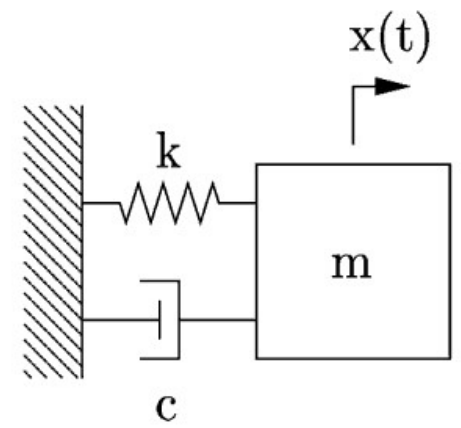

Figure A.5: Spring-mass-damper system. ${ }^{61}$ 
the forces can again be summed in the x-direction to yield

$$
m \ddot{x}(t)+c \dot{x}(t)+k x(t)=0
$$

as the new equation of motion of the spring-mass-damper system. An alternative form of this EOM can be written as

$$
m \lambda^{2}+c \lambda+k=0
$$

and is referred to as the characteristic equation of the system.

By applying the quadratic formula to this form of the EOM, two solutions arise as

$$
\lambda_{1,2}=-\frac{c}{2 m} \pm \frac{1}{2 m} \sqrt{c^{2}-4 k m}
$$

where $\lambda_{1}$ and $\lambda_{2}$ represent the roots of the solution. The discriminant $c^{2}-4 k m$ also indicates the nature of the two roots, and will be discussed below in further detail. It should be noted that it is convenient at this point to define the critical damping coefficient $c_{c r}$ as

$$
c_{c r}=2 m \omega_{n}=2 \sqrt{k m}
$$

and used to define an important parameter known as the damping ratio $\zeta$ where

$$
\zeta=\frac{c}{c_{c r}}=\frac{c}{2 m \omega_{n}}=\frac{c}{2 \sqrt{k m}}
$$

which is a critical parameter in any vibration study or model. Not only does $\zeta$ help determine if the roots of the characteristic equation will be real or complex, but it also determines the damping nature of a given system, which is an otherwise difficult task to achieve with a pure analytic approach.

Coming back to the discriminant $c^{2}-4 k m$, three separate cases are possible. In first case where the discriminant is negative, the roots are a complex conjugate pair. As 
such, $\zeta$ will be less than 1 in this case. The two root solutions can be re-written as

$$
\lambda_{1,2}=-\zeta \omega_{n} \pm \sqrt{1-\zeta^{2}}
$$

and yield

$$
x(t)=A e^{-\zeta \omega_{n} t} \sin \left(\omega_{d} t+\phi\right), \text { where } \omega_{d}=\omega_{n} \sqrt{1-\zeta^{2}}
$$

Here, $x(t)$ is the solution of the underdamped system and $\omega_{d}$ represents the damped natural frequency of the system.

By applying the initial conditions $x_{0}$ and $v_{0}$ to

$$
A=\sqrt{\frac{\left(v_{0}+\zeta \omega_{n} x_{0}\right)^{2}+\left(x_{0} \omega_{d}\right)^{2}}{\omega_{d}^{2}}}, \quad \phi=\tan ^{-1} \frac{x_{0} \omega_{d}}{v_{0}+\zeta \omega_{n} x_{0}}
$$

both the amplitude $A$ and phase $\phi$ can be calculated for a complete solution $x(t)$ of the underdamped system.

In the second case where the discriminant $c^{2}-4 k m$ is positive, the roots are a distinct real pair. As such, $\zeta$ will be greater than 1 for this case. The two root solutions can be re-written as

$$
\lambda_{1,2}=-\zeta \omega_{n} \pm \omega_{n} \sqrt{\zeta^{2}-1}
$$

to yield

$$
x(t)=e^{-\zeta \omega_{n} t}\left(a_{1} e^{-\left(\omega_{n} \sqrt{\zeta^{2}-1}\right) t}+a_{2} e^{+\left(\omega_{n} \sqrt{\zeta^{2}-1}\right) t}\right)
$$

where $x(t)$ is the solution of the overdamped system. By applying the initial conditions $x_{0}$ and $v_{0}$ to

$$
a_{1}=\frac{-v_{0}+\left(-\zeta+\sqrt{\zeta^{2}-1}\right) \omega_{n} x_{0}}{2 \omega_{n} \sqrt{\zeta^{2}-1}}, \quad a_{2}=\frac{v_{0}+\left(\zeta+\sqrt{\zeta^{2}-1}\right) \omega_{n} x_{0}}{2 \omega_{n} \sqrt{\zeta^{2}-1}}
$$


both constants of integration $a_{1}$ and $a_{2}$ can be calculated for a complete solution $x(t)$ of the overdamped system.

In the third case where the discriminant $c^{2}-4 k m$ is zero, the roots are a repeated pair. As such, $\zeta$ will be equal to 1 for this case. The roots then become

$$
\lambda_{1}=\lambda_{2}=-\omega_{n}
$$

to yield

$$
x(t)=\left(a_{1}+a_{2} t\right) e^{-\omega_{n} t}
$$

where $x(t)$ is the solution of the critically damped system. Again, by applying the initial conditions $x_{0}$ and $v_{0}$ to

$$
a_{1}=x_{0}, \quad a_{2}=v_{0}+\omega_{n} x_{0}
$$

both constants of integration $a_{1}$ and $a_{2}$ can be calculated for a complete solution $x(t)$ of the critically damped system.

As noted in the previous three cases, the damping ratio $\zeta$ determines whether a system is underdamped $(\zeta<1)$, overdamped $(\zeta>1)$, or critically damped $(\zeta=1)$ (see Figure A.6). For undamped systems, $\zeta$ is simply zero and it can be verified by letting $\zeta$ be zero in the solution $x(t)$ of an underdamped system (Equation A.14). It can be shown that the equation will reduce to the solution of an undamped system (Equation A.6). 


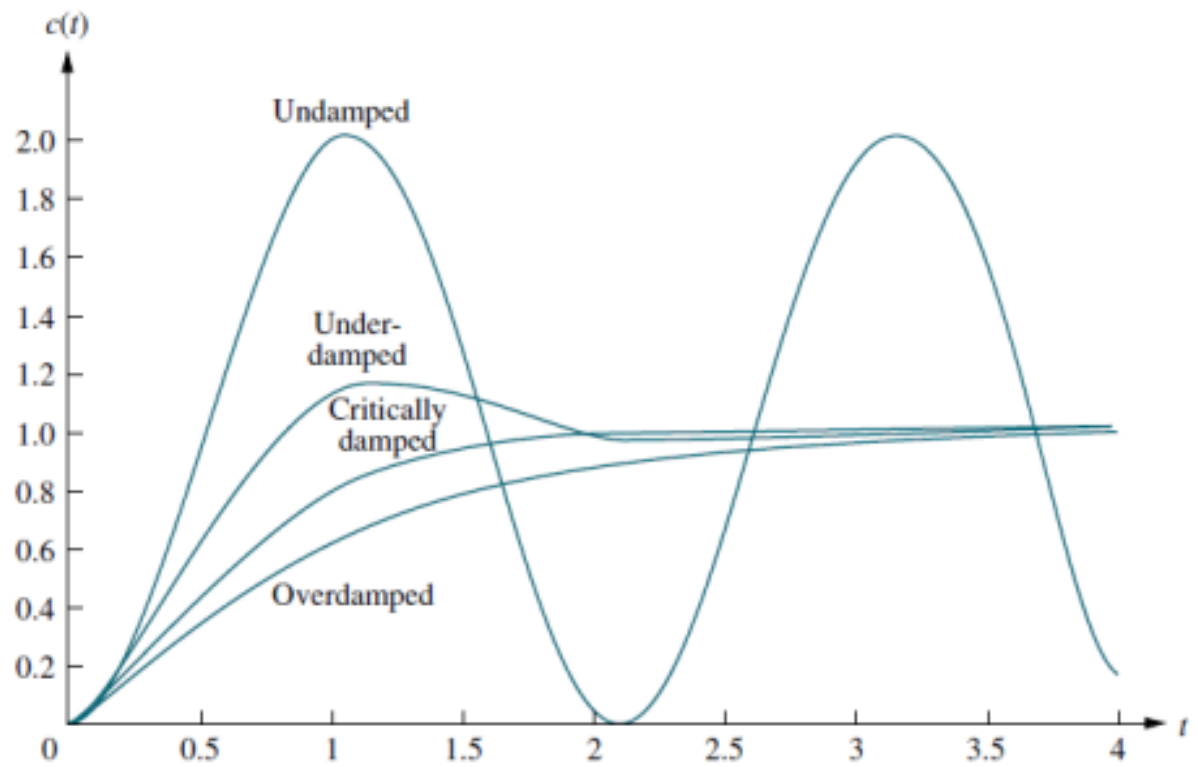

Figure A.6: Response overlay with various damping ratios. ${ }^{75}$ 


\section{A.3 Stiffness}

The concept of spring stiffness $k$ can be extended to other applications like structural components. If a small section is cut from the spring body for example, this can be similar to analyzing a section of a rod with a defined length $l$, cross sectional area $A$, and material elastic modulus $E$. These properties can then be used to define the stiffness as

$$
k=\frac{E A}{l}
$$

where $k$ is the vibration stiffness along the longitudinal axis of the bar (see Figure A.7).

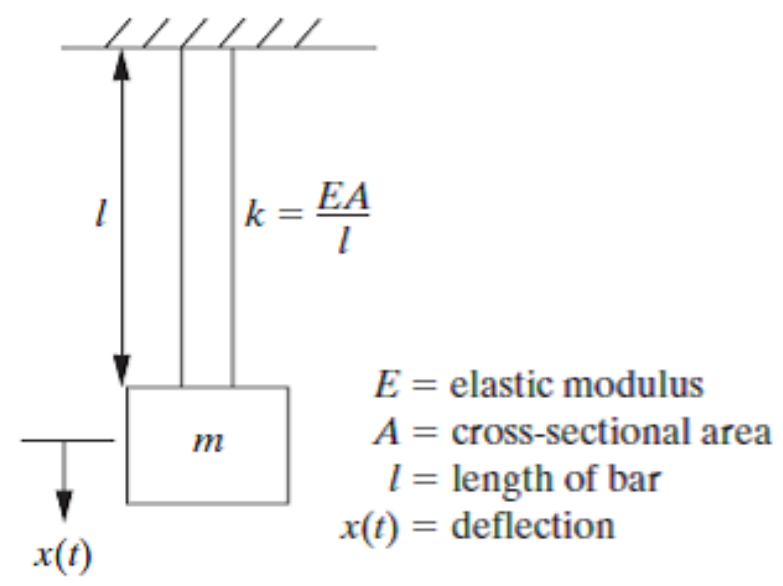

Figure A.7: Stiffness of a bar along the longitudinal axis. ${ }^{84}$

Similarly, the stiffness of a beam under transverse loading can be defined as

$$
k=\frac{3 E I}{l^{3}}
$$

where $k$ is the vibration stiffness along the transverse axis of the beam (see Figure A.8) and $I$ is the area moment of inertia of the beam. For this case, only the tip mass $m$ is considered while the mass of the beam is assumed to be negligible. 


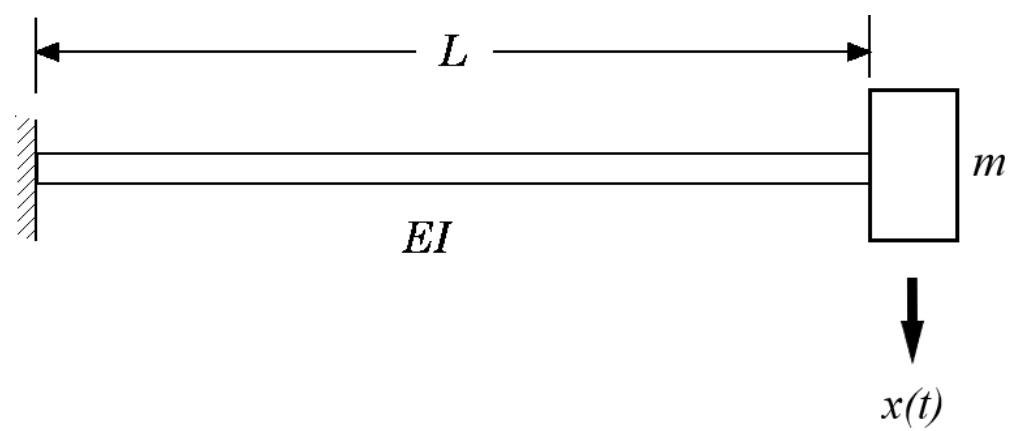

Figure A.8: Stiffness of a bar along the longitudinal axis. ${ }^{55}$

With the vibration stiffness defined for the beam, it is now possible to deduce the natural frequency of the tip mass as

$$
\omega_{n}=\sqrt{\frac{k}{m}}=\sqrt{\frac{3 E I}{m l^{3}}}
$$

where it will continue to oscillate in the same direction as $x(t)$.

In some cases, beam vibration may require the analysis to include the mass of the beam in order to determine its effects on the dynamics of the structure. An example of such analysis is the vibration of an airplane wing with an attached engine or fuel tank. For this analysis, the classic equation for static deflection of a beam is applied to the energy equation of a beam to yield

$$
m_{e q}=\frac{33}{140} M+m
$$

where $m_{e q}$ is the equivalent mass of the system. In the case of the airplane wing, $M$ would represent the mass of the wing and $m$ the mass of the engine or fuel tank. With an equivalent mass defined, the natural frequency $\omega_{n}$ of the beam would now be

$$
\omega_{n}=\sqrt{\frac{k}{\frac{33}{140} M+m}}
$$

which can be weighed against the negligible beam mass analysis. 


\section{A.4 Coulomb Friction}

Up to this point, spring-mass models discussed here have neglected the effects of friction as a way to simplify the analysis. However, friction is an inherent force of many vibration systems in nature, and it should be accounted for in the analysis as necessary.

From physics, recall that friction opposes the force acting on the block (see Figure A.9) and is defined as

$$
f=-\mu N=-\mu m g
$$

where $\mu$ is the coefficient of friction at the block/ground interface, $g$ is gravity, and $N$ is the normal force which opposes the weight $m g$ of the block.

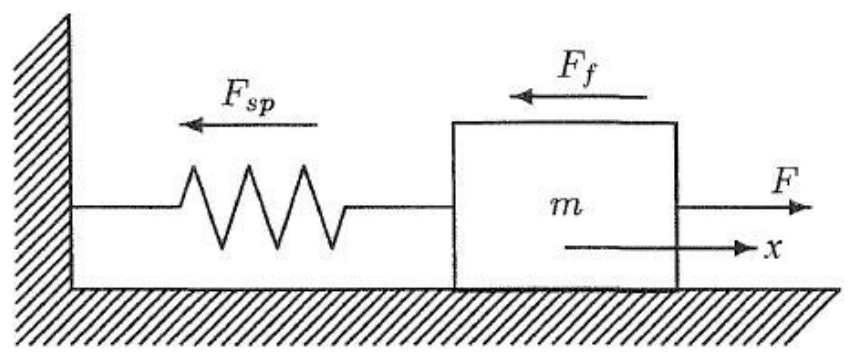

\section{Figure A.9: Spring-mass model with sliding friction. ${ }^{36}$}

The standard EOM for a spring-mass system

$$
m \ddot{x}+k x=0
$$

can now be modified by summing forces over the $\mathrm{x}$-direction to yield

$$
m \ddot{x}+k x=-\mu m g
$$

as the new EOM for a spring-mass system with friction. 
The coefficient of friction $\mu$ generally depends on the materials in contact due to the nature of their surfaces, with some having rougher surfaces than others. The condition of the surfaces (e.g. dry, lubricated) also influences the friction factor, and should be accounted for in any analysis as necessary. The static coefficient of friction $\mu_{s}$ corresponds from the rest position to the point just prior to relative movement, while the dynamic coefficient of friction $\mu_{k}$ takes over once motion has initiated. Examples of various material contacts and their corresponding coefficient of friction can be found in Table A.1.

Table A.1: Coefficient of friction for various materials in contact. ${ }^{26}$

\begin{tabular}{|l|c|c|}
\hline \multicolumn{1}{|c|}{ Materials } & $\boldsymbol{\mu}_{\mathbf{s}}$ & $\boldsymbol{\mu}_{\mathbf{k}}$ \\
\hline Aluminum on aluminum & 1.05 & 1.4 \\
\hline Steel on steel & 0.74 & 0.57 \\
\hline Aluminum on steel & 0.61 & 0.47 \\
\hline Copper on steel & 0.53 & 0.36 \\
\hline Rubber on concrete & 1.0 & 0.8 \\
\hline Lubricated metal on metal & 0.15 & 0.06 \\
\hline Ice on ice & 0.1 & 0.03 \\
\hline Teflon on teflon & 0.04 & 0.04 \\
\hline Synovial joint of humans & 0.01 & 0.003 \\
\hline
\end{tabular}

From the new EOM for the spring-mass system with friction (Equation A.29), it can be shown that the equation has the solution

$$
x(t)=A_{1} \cos \omega_{n} t+B_{1} \sin \omega_{n} t+\frac{\mu m g}{k}
$$

where $A_{1}$ and $B_{1}$ are constants that can be determined from the initial conditions of the system.

There are a few things to note about Coulomb friction damping when compared to viscous damping (see Appendix A.2 for more details). For instance, unlike viscous damping where the response decays exponentially, damping from Coulomb friction decays with slope 


$$
-\frac{2 \mu m g \omega_{n}}{\pi k}
$$

which is linear and not exponential. ${ }^{84}$ Furthermore, Coulomb friction damping brings a system to rest, but not necessarily to the same position as when it started; as opposed to a system with viscous damping which continues to oscillate around the same equilibrium point with a small amplitude. Lastly, the oscillating and undamped frequencies are the same in a system with Coulomb friction, while viscous damping changes the oscillating frequency of the system.

Implementing friction into the analysis of a vibration model can prove to be challenging, as friction itself has a nonlinear behavior, making it difficult to derive analytical solutions. Vibration problems with nonlinear behavior (see Figure A.10) are more complex since friction adds many possible equilibrium points, unlike the single point of equilibrium for linear systems with negligible friction. ${ }^{84}$

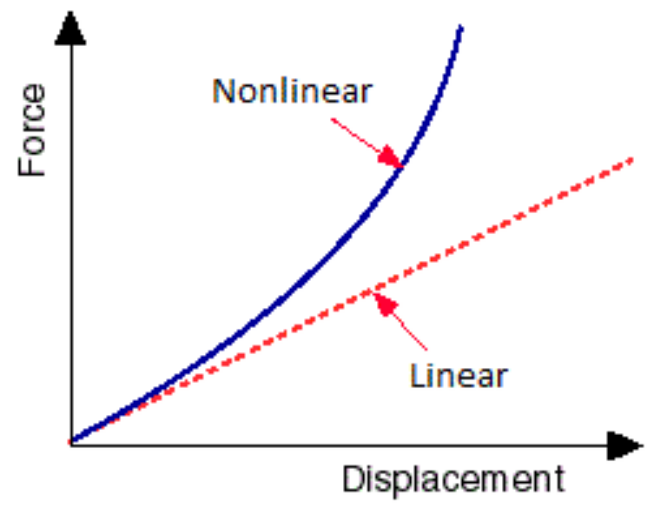

Figure A.10: Force vs displacement curve of a linear and nonlinear system. ${ }^{17}$ 


\section{A.5 Forced Vibration of Undamped Systems}

While the previous sections discussed systems in free vibration, this section now focuses on the harmonic excitation of spring-mass systems, also known as forced vibration (see Figure A.11). In the analysis of a forced vibration model, close attention is required when comparing the natural frequency of the system and the driving frequency of the applied force. As the driving frequency approaches the natural frequency of the system, a phenomena known as resonance develops, which can be destructive for a vibration system to the point of collapse and failure.

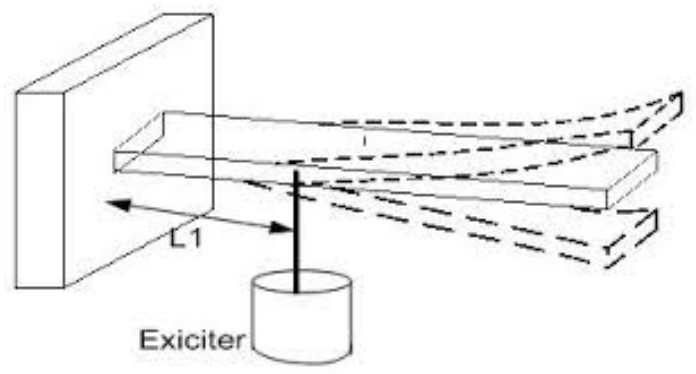

Figure A.11: Vibration of a cantilever beam using an excitation force. ${ }^{37}$

Starting with the case of an undamped spring-mass system (see Figure A.12),

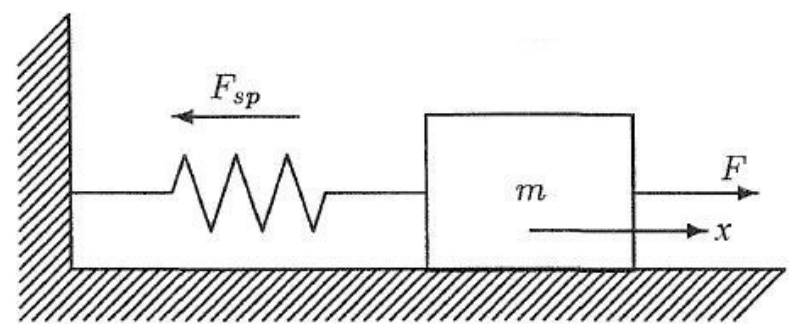

Figure A.12: Spring-mass model with force $F$ and negligible friction. ${ }^{36}$

assume that the applied force $F$ has a form

$$
F(t)=F_{0} \cos \omega t
$$


where $F_{0}$ and $\omega$ in this expression represent the magnitude and driving frequency, respectively, of the applied force. By creating a free body diagram of Figure A.12 and summing forces in the x-direction, it can be shown that the EOM of the system is

$$
m \ddot{x}(t)+k x(t)=F_{0} \cos \omega t
$$

In standard form,

$$
\ddot{x}(t)+\omega_{n}^{2} x(t)=f_{0} \cos \omega t
$$

where $f_{0}$ is referred to as the mass-normalized force.

By applying the principles of differential equations, the solution to Equation A.34 is the combination of both a homogeneous and particular solution such that

$$
x(t)=x_{h}(t)+x_{p}(t)
$$

where

$$
x_{p}(t)=\frac{f_{0}}{\omega_{n}^{2}-\omega^{2}} \cos \omega t
$$

and

$$
x_{h}(t)=A \sin \left(\omega_{n} t+\phi\right)=A_{1} \sin \omega_{n} t+A_{2} \cos \omega_{n} t
$$

Initial conditions can be applied to

$$
A_{1}=\frac{v_{0}}{\omega_{n}}, \quad A_{2}=x_{0}-\frac{f_{0}}{\omega_{n}^{2}-\omega^{2}}
$$

to determine constants $A_{1}$ and $A_{2}$. Together, the completed solution $x(t)$ becomes

$$
x(t)=\frac{v_{0}}{\omega_{n}} \sin \omega_{n} t+\left(x_{0}-\frac{f_{0}}{\omega_{n}^{2}-\omega^{2}}\right) \cos \omega_{n} t+\frac{f_{0}}{\omega_{n}^{2}-\omega^{2}} \cos \omega t
$$




\section{A.6 Forced Vibration of Damped Systems}

Similar to the previous analysis of undamped spring-mass systems under harmonic excitation, the focus now shifts to such systems that incorporate damping elements. Taking the spring-mass model with a damping element $c$ (see Figure A.13),

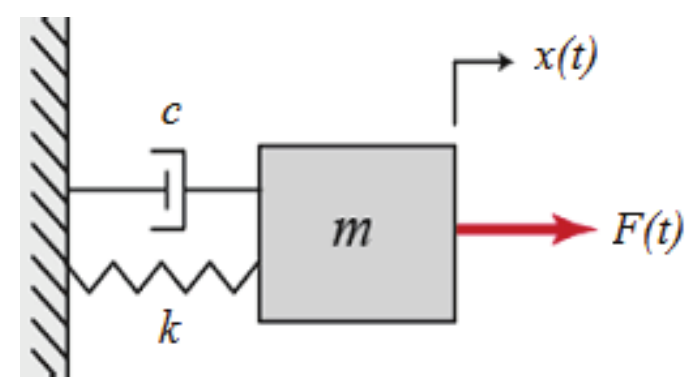

Figure A.13: Spring-mass-damper model with applied force $\boldsymbol{F} .{ }^{44}$

the free body diagram (FBD) for this model yields

$$
m \ddot{x}+c \dot{x}+k x=F_{0} \cos \omega t
$$

as the EOM for a spring-mass-damper system under harmonic excitation. In standard form,

$$
\ddot{x}+2 \zeta \omega_{n} \dot{x}+\omega_{n}^{2} x=f_{0} \cos \omega t
$$

where $c$ is the damping coefficient of the damper element. By applying the principles of differential equations, the solution to the above EOM is a combination of a homogeneous and particular solution where the latter takes the form

$$
x_{p}(t)=X \cos (\omega t-\theta)
$$

By applying the method of undetermined coefficients, it can be shown that

$$
X=\frac{f_{0}}{\sqrt{\left(\omega_{n}^{2}-\omega^{2}\right)^{2}+\left(2 \zeta \omega_{n} \omega\right)^{2}}}, \quad \theta=\tan ^{-1} \frac{2 \zeta \omega_{n} \omega}{\omega_{n}^{2}-\omega^{2}}
$$


where $X$ and $\theta$ are coefficients of the particular solution $x_{p}$. Together, the particular solution becomes

$$
x_{p}(t)=\frac{f_{0}}{\sqrt{\left(\omega_{n}^{2}-\omega^{2}\right)^{2}+\left(2 \zeta \omega_{n} \omega\right)^{2}}} \cos \left(\omega t-\tan ^{-1} \frac{2 \zeta \omega_{n} \omega}{\omega_{n}^{2}-\omega^{2}}\right)
$$

Recall that

$$
x(t)=x_{h}(t)+x_{p}(t)
$$

where the homogeneous solution

$$
x_{h}(t)=A e^{-\zeta \omega_{n} t} \sin \left(\omega_{d} t+\phi\right)
$$

is the same as the previously derived solution for an underdamped system in free vibration. By applying initial conditions to $x(t)$,

$$
A=\frac{x_{0}-X \cos \theta}{\sin \phi}, \quad \phi=\tan ^{-1} \frac{\omega_{d}\left(x_{0}-X \cos \theta\right)}{v_{0}+\left(x_{0}-X \cos \theta\right) \zeta \omega_{n}-\omega X \sin \theta}
$$

where $A$ and $\phi$ are the amplitude and phase, respectively, of the homogeneous solution $x_{h}(t)$ in Equation A.46. Combining all terms,

$$
\begin{aligned}
x(t)=\frac{x_{0}-X \cos \theta}{\sin \phi} & e^{-\zeta \omega_{n} t} \sin \left(\omega_{d} t+\tan ^{-1} \frac{\omega_{d}\left(x_{0}-X \cos \theta\right)}{v_{0}+\left(x_{0}-X \cos \theta\right) \zeta \omega_{n}-\omega X \sin \theta}\right) \\
& +\frac{f_{0}}{\sqrt{\left(\omega_{n}^{2}-\omega^{2}\right)^{2}+\left(2 \zeta \omega_{n} \omega\right)^{2}}} \cos \left(\omega t-\tan ^{-1} \frac{2 \zeta \omega_{n} \omega}{\omega_{n}^{2}-\omega^{2}}\right)
\end{aligned}
$$

where $x(t)$ is the solution to a spring-mass-damper system under harmonic excitation. Note that

$$
x_{h}(t) \rightarrow 0 \quad \text { as } \quad t \rightarrow \infty
$$

and so $x(t)=x_{p}(t)=x_{s s}(t)$, where $x_{s s}(t)$ is known as the steady-state solution and $x_{h}(t)$ the transient solution of the response. In general, $x_{s s}(t)$ is regarded to be more important since it prevails for a longer period of time, as opposed to $x_{h}(t)$ which dies off sooner. ${ }^{84}$ However, deciding to ignore the effects of the transient solution $x_{h}(t)$ should ultimately be based on careful engineering judgment. 


\section{A.7 Vibration of Membranes and Plates}

Displacement of membranes and plates is a function of two directions, as opposed to the single direction of planar bars and beams for example. Their response to dynamic loading lead to modes of vibration that propagate throughout their surface in both directions simultaneously, creating intricate shapes and features that help identify areas in the design that require special attention or modification.

Membranes are thin elements, and as such, have no real resistance to bending. However, membranes do have strength in tension, similar to a string, and can deform based on the type of loading and boundary conditions of the membrane system (see Figure A.14).

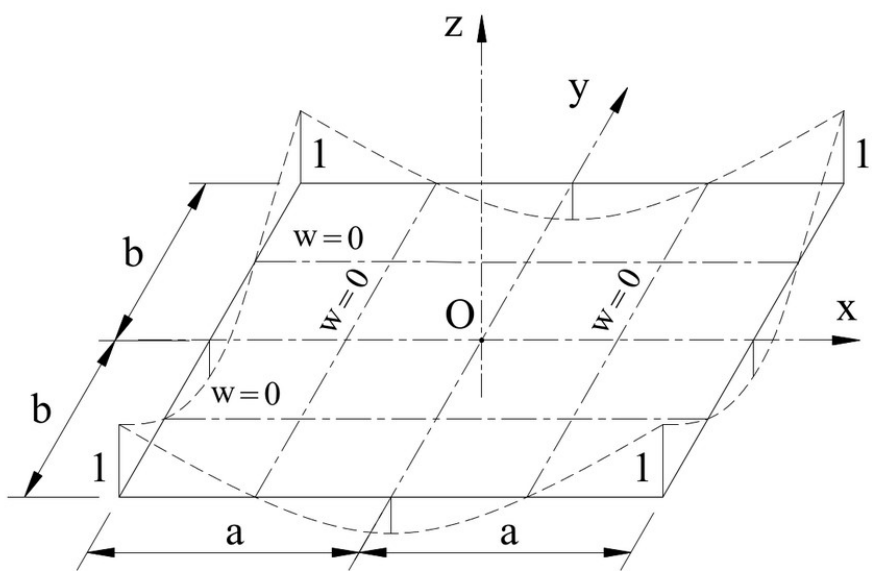

Figure A.14: Mode shape of a vibrating membrane with displacement $w .^{18}$

Letting $w$ represent the displacement in the $\mathrm{x}-\mathrm{y}$ directions over time $t$, it can be shown that the equation of free vibration for a membrane becomes

$$
\tau \nabla^{2} w(x, y, t)=\rho w_{t t}(x, y, t)
$$

where $\tau$ is the constant tension per length, $\nabla^{2}$ the Laplace operator, and $\rho$ the mass per unit area of the membrane. 
Using the rectangular form of the Laplace operator

$$
\nabla^{2}=\frac{\partial^{2}}{\partial x^{2}}+\frac{\partial^{2}}{\partial y^{2}}
$$

Equation A.49 can be re-written as

$$
\frac{\partial^{2} w(x, y, t)}{\partial x^{2}}+\frac{\partial^{2} w(x, y, t)}{\partial y^{2}}=\frac{1}{c^{2}} \frac{\partial^{2} w(x, y, t)}{\partial t^{2}}, \quad \text { where } \quad c=\sqrt{\frac{\tau}{\rho}}
$$

Unlike membranes, plate elements have significant thickness which allows them to resist bending moments, and their modes are again a combination of two directions (see Figure A.15). The main assumptions are that normal stresses in the transverse direction of the plate are assumed negligible, and that there is no significant change in thickness of the plate while under loading. ${ }^{84}$

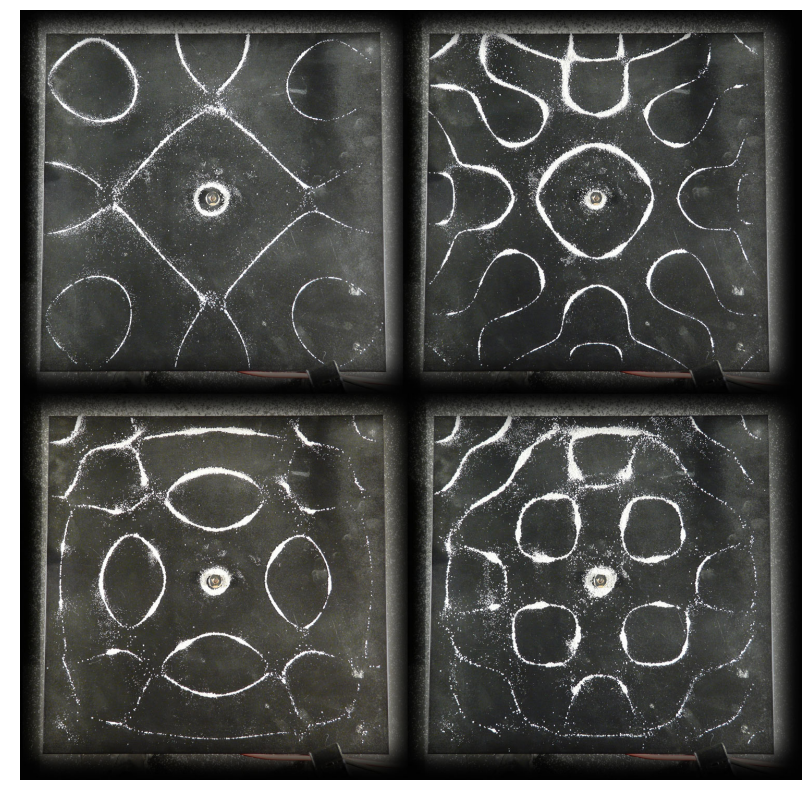

Figure A.15: Sand outlining mode shapes of a vibrating plate at four different frequencies. ${ }^{25}$ 
In a similar analysis to membranes, in can be shown that the equation of free vibration for a plate becomes

$$
-D_{E} \nabla^{4} w(x, y, t)=\rho w_{t t}(x, y, t)
$$

where $D_{E}$ is the plate flexural rigidity, $\nabla^{4}$ the biharmonic operator, $E$ the elastic modulus, and $\rho$ the mass density of the material.

The plate flexural rigidity $D_{E}$ is defined as

$$
D_{E}=\frac{E h^{3}}{12\left(1-\nu^{2}\right)}
$$

where $h$ is the thickness of the plate and $\nu$ is the Poisson's ratio of the plate material.

Using the rectangular form of the biharmonic operator

$$
\nabla^{4}=\frac{\partial^{4}}{\partial x^{4}}+2 \frac{\partial^{4}}{\partial x^{2} \partial y^{2}}+\frac{\partial^{4}}{\partial y^{4}}
$$

Equation A.52 can be re-written to include the boundary conditions of the plate system (not derived here). A clamped edge of a square plate for example, would experience no deflection where

$$
w(x, y, t)=0
$$

and together with the remaining boundary conditions, a solution can be developed that describes the behavior of a plate under dynamic loading. 
Appendix B

FINITE ELEMENT THEORY

\section{B.1 Stiffness (Displacement) Method}

There are two general approaches to solving problems using the finite element method (FEM). The first approach is the force (flexibility) method which solves for the unknown internal forces of the elements in a structure, and the second approach is the stiffness (displacement) method which solves for the unknown displacements of the elements in the structure. ${ }^{85}$ The latter method is easier to work with mathematically and has also been adopted by most FEM software programs out in the market. As such, the subsequent sections will only focus around the displacement method.

\section{B.1.1 Spring Element}

Springs are perhaps the most fundamental elements used to understand how the displacement method is applied (see Figure B.1). Here, a linear behavior is assumed where the tension load $T$ and displacement $u$ act along the axis of the spring.

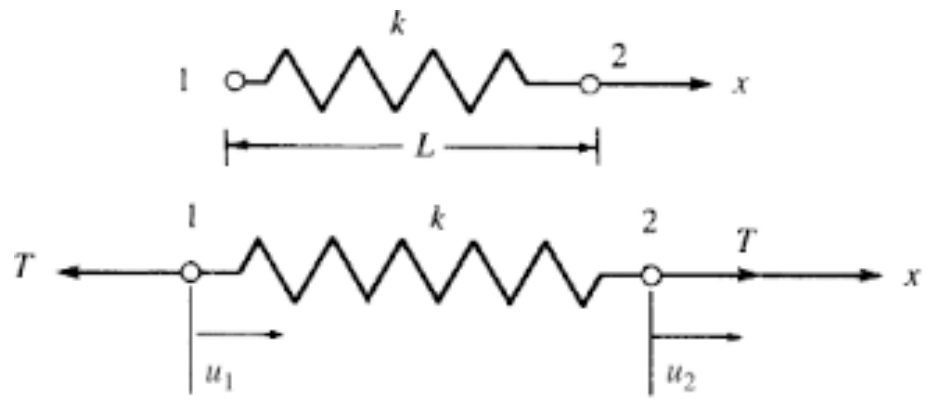

Figure B.1: Linear spring element with tension force $T$ and nodal displacement $u .^{85}$ 
Recall from vibration theory that the spring force $f$ is equal to $k x$. Similarly,

$$
\{f\}=[k]\{d\}
$$

where $\{f\}$ is the nodal force matrix, $[k]$ is the stiffness matrix, and $\{d\}$ is the nodal displacement matrix of the spring element.

For a structure or assembly with multiple spring elements, it is necessary to change Equation B.1 to

$$
\{F\}=[K]\{d\}
$$

where the terms are now presented in global coordinates. Reverting to the spring element in Figure B.1, it can be shown that

$$
\left\{\begin{array}{l}
f_{1 x} \\
f_{2 x}
\end{array}\right\}=\left[\begin{array}{ll}
k_{11} & k_{12} \\
k_{21} & k_{22}
\end{array}\right]\left\{\begin{array}{l}
u_{1} \\
u_{2}
\end{array}\right\}
$$

which describes the node properties of the spring element in local coordinates. If two pieces of information are available, then it is possible to solve for the unknown in most cases. Again, for systems with multiple elements, it is necessary to combine elements in local coordinates and build towards global coordinates in order to analyze the behavior of the system as a whole.

\section{B.1.2 Bar Element}

With the fundamentals of the displacement method now established (see Appendix B.1 for more details), it is worthwhile to discuss bar elements. Many large structures can be analyzed using bar elements, including truss bridges and radio towers. The assumption again is that the force acts only along the axis of the bar element, whether the bar element is in tension or compression (see Figure B.2). This section will only cover 2D bar elements, as the derivations for 3D bar elements are more involved, yet still sharing the same principles. 

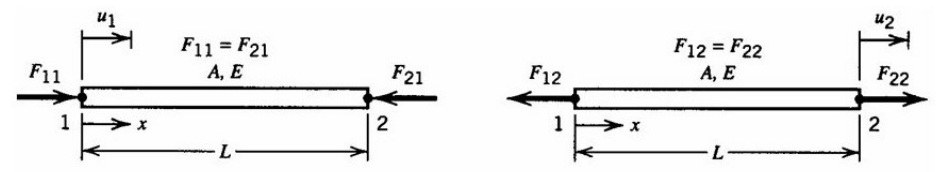

\section{Figure B.2: Bar element under axial loading $F .^{36}$}

Aside from nodal displacement $u$, bar elements can also be used to solve for the nodal stress $\sigma$ and strain $\varepsilon$ of the element where

$$
\sigma_{x}=E \varepsilon_{x}
$$

and

$$
\varepsilon_{x}=\frac{d u}{d x}
$$

Note that for bar elements,

$$
A \sigma_{x}=T=\text { constant }
$$

where $A$ is the constant cross-sectional area of the bar and $T$ is the applied load. Reverting to Figure B.2, it can be shown that

$$
\left\{\begin{array}{l}
f_{1 x} \\
f_{2 x}
\end{array}\right\}=\frac{A E}{L}\left[\begin{array}{rr}
1 & -1 \\
-1 & 1
\end{array}\right]\left\{\begin{array}{l}
u_{1} \\
u_{2}
\end{array}\right\}
$$

where

$$
[k]=\frac{A E}{L}\left[\begin{array}{rr}
1 & -1 \\
-1 & 1
\end{array}\right]
$$

is the stiffness matrix of a bar element in local coordinates, $E$ is the elastic modulus of the material, and $L$ is length of the bar element. For the case where the local bar element is arbitrarily oriented in a plane with angle $\theta$, it is necessary to apply

$$
[T]=\left[\begin{array}{rr}
C & S \\
-S & C
\end{array}\right] \quad(C=\cos \theta, S=\sin \theta)
$$

where $[T]$ is the transformation matrix between two coordinate systems and

$$
[k]=\frac{A E}{L}\left[\begin{array}{cccc}
C^{2} & C S & -C^{2} & -C S \\
& S^{2} & -C S & -S^{2} \\
& C^{2} & C S \\
\text { Symmetry } & & S^{2}
\end{array}\right]
$$


is the stiffness matrix of an arbitrarily oriented bar element in global coordinates.

As previously mentioned, it is possible to solve for the axial stress $\sigma$ in a structure or assembly composed of bar elements. Here,

$$
\{\sigma\}=\left[C^{\prime}\right]\{d\}
$$

where

$$
\left[C^{\prime}\right]=\frac{E}{L}\left[\begin{array}{llll}
-C & -S & C & S
\end{array}\right]
$$

and $\{d\}$ is again the nodal displacement matrix in local coordinates.

Bar elements have many applications in the field of the engineering and can help simplify the analysis of a system (see Figure B.3), especially if the analysis can take advantage of symmetry which will significantly reduce the number of calculations required to reach a solution.

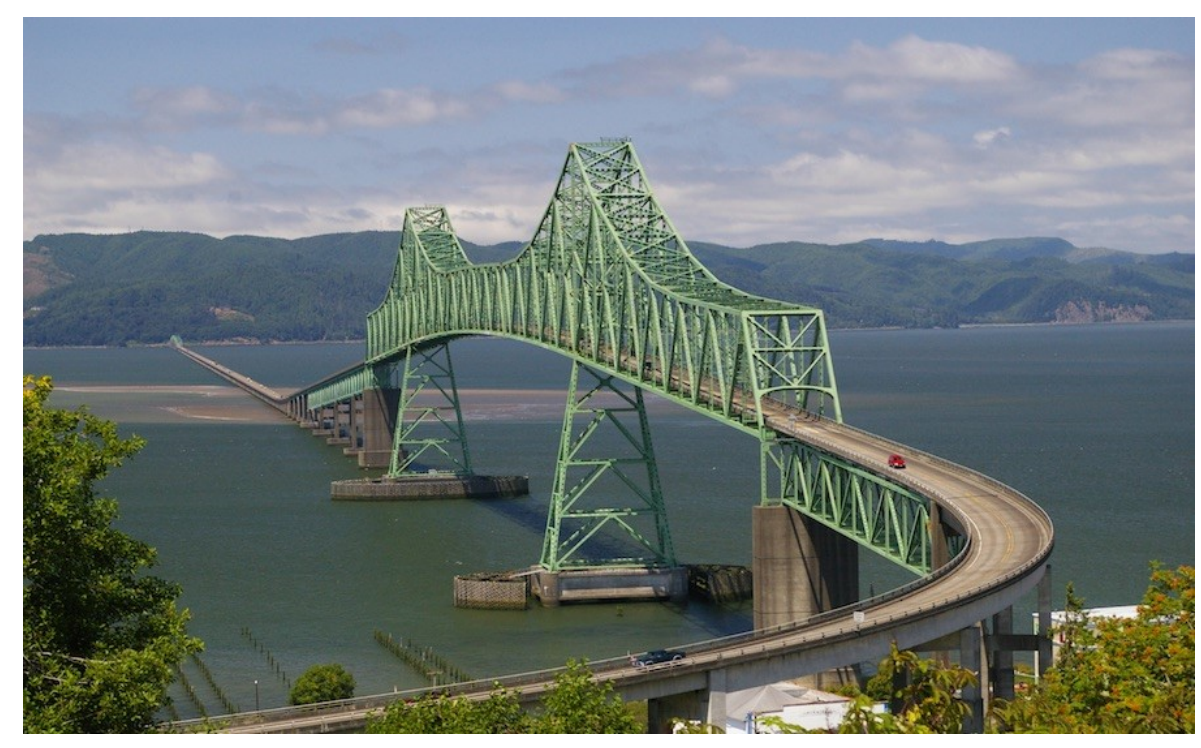

Figure B.3: Truss bridges can be analyzed using bar elements. ${ }^{83}$ 


\section{B.1.3 Beam Element}

Unlike bar elements which are limited to axial loading, beam elements can model transverse loading and the subsequent bending motion (see Figure B.4). Beam elements are applicable to many important systems, including the beam and column framework of most structural buildings. This section will only cover $2 \mathrm{D}$ beam elements, as the derivations for 3D beam elements are more involved, yet still sharing the same principles.

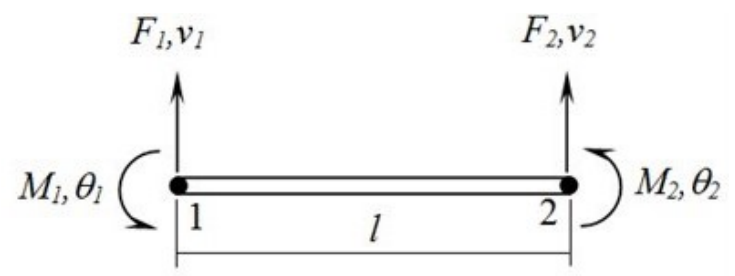

\section{Figure B.4: Beam element under transverse loading. ${ }^{36}$}

Recall from mechanics of materials that the beam bending stress $\sigma$ can be represented as

$$
\sigma_{x}=\frac{-M y}{I}
$$

where $M$ is the bending moment, $y$ is the transverse displacement, and $I$ is the area moment of inertia of the beam cross section. Together with the bending $m$ and shear force $V$ relationships

$$
m(x)=E I \frac{\partial^{2} v}{\partial x^{2}} \quad, \quad V=E I \frac{\partial^{3} v}{\partial x^{3}}
$$

it can be shown that

$$
\left\{\begin{array}{l}
f_{1 y} \\
m_{1} \\
f_{2 y} \\
m_{2}
\end{array}\right\}=\frac{E I}{L^{3}}\left[\begin{array}{rrrr}
12 & 6 L & -12 & 6 L \\
6 L & 4 L^{2} & -6 L & 2 L^{2} \\
-12 & -6 L & 12 & -6 L \\
6 L & 2 L^{2} & -6 L & 4 L^{2}
\end{array}\right]\left\{\begin{array}{l}
v_{1} \\
\phi_{1} \\
v_{2} \\
\phi_{2}
\end{array}\right\}
$$

where $f$ is the transverse nodal force, $m$ is the bending moment at the node, $v$ is transverse nodal displacement, and $\phi$ is the nodal rotation. This equation applies to 
long and slender beams where transverse shear is assumed to be negligible. ${ }^{85}$ Deducing from Equation B.15, the stiffness matrix $k$ for a long slender beam is represented by

$$
[k]=\frac{E I}{L^{3}}\left[\begin{array}{rrrr}
12 & 6 L & -12 & 6 L \\
6 L & 4 L^{2} & -6 L & 2 L^{2} \\
-12 & -6 L & 12 & -6 L \\
6 L & 2 L^{2} & -6 L & 4 L^{2}
\end{array}\right]
$$

For cases where transverse shear cannot be ignored (beams with small length to depth ratios), it is necessary to modify the stiffness matrix $k$ in order to account for the effects of shear stress. To do this, the non-dimensional shear correction factor $\varphi$ is defined as

$$
\varphi=\frac{12 E I}{k_{s} A G L^{2}}=\frac{12 g}{L^{2}}
$$

where $k_{s} A$ is the shear area, $G$ is the shear modulus, and $g$ is the transverse shear term. Together, the stiffness matrix for a beam with transverse shear becomes

$$
[k]=\frac{E I}{L^{3}(1+\varphi)}\left[\begin{array}{cccc}
12 & 6 L & -12 & 6 L \\
6 L & (4+\varphi) L^{2} & -6 L & (2-\varphi) L^{2} \\
-12 & -6 L & 12 & -6 L \\
6 L & (2-\varphi) L^{2} & -6 L & (4+\varphi) L^{2}
\end{array}\right]
$$

Beam element analysis is applicable to many systems, and can be a powerful tool when trying to develop solutions for structural dynamic problems (see Figure B.5). Like bar elements, beam elements can take advantage of symmetry in a model to reduce computation time.

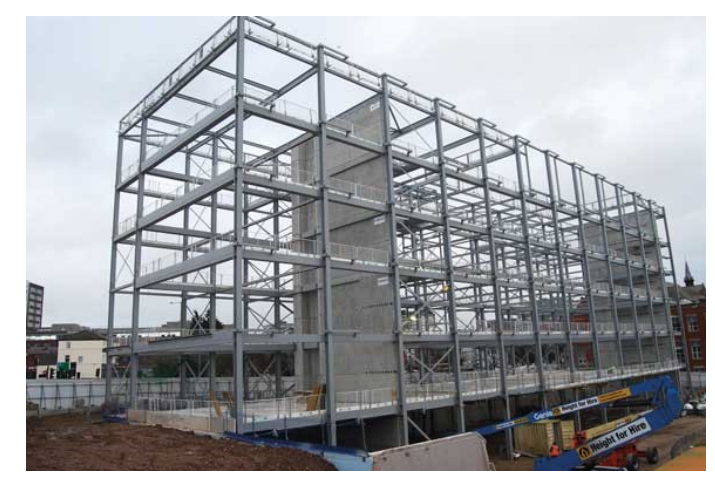

Figure B.5: Building designs require dynamic analysis to check for potential structural damage from earthquakes. ${ }^{36}$ 


\section{B.2 Frame and Grid Stiffness}

Many systems can be analyzed using a combination of beam sub-assemblies known as frames and grids. Beam elements can be modified to model transverse and axial loads, along with the respective bending moments (see Figure B.6). This makes beam elements quite versatile for the analysis of many structures, and combining them in frame and grid systems can help simplify the analysis of such structures. This discussion will focus only on frames and grids in 2D space, as it will become evident that even their 2D matrix solutions can become quite elaborate.

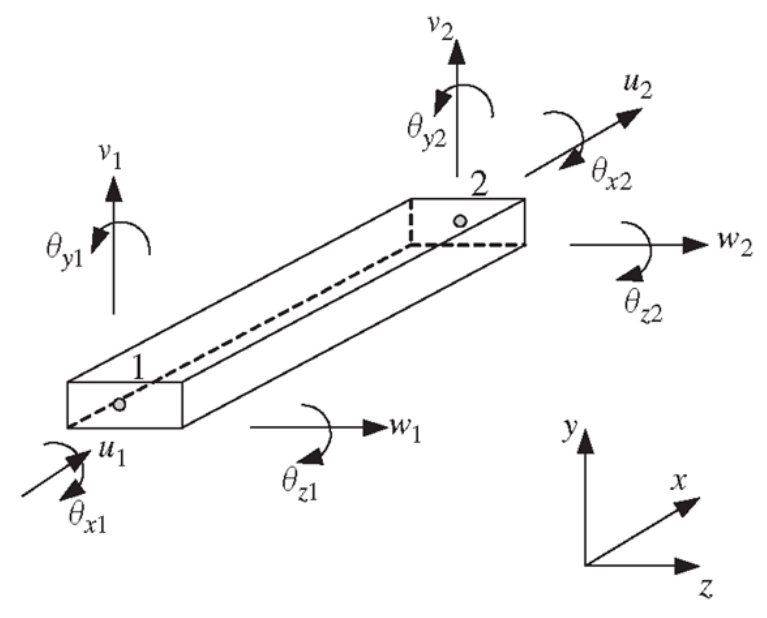

Figure B.6: Example of a 6-DOF beam element used in frame analysis. ${ }^{35}$

For plane frames, loads are applied in-plane of the frame structure (see Figure B.7), where only the axial displacement $u$, transverse displacement $v$, and rotation $\phi$ at the beam element nodes will be considered for this case. It should be noted that the joints of plane frames discussed here are assumed to be rigid, where the angles between elements remain unchanged after loading, and bending moments can be transferred from one element to another for moment continuity at the joints. ${ }^{85}$ 


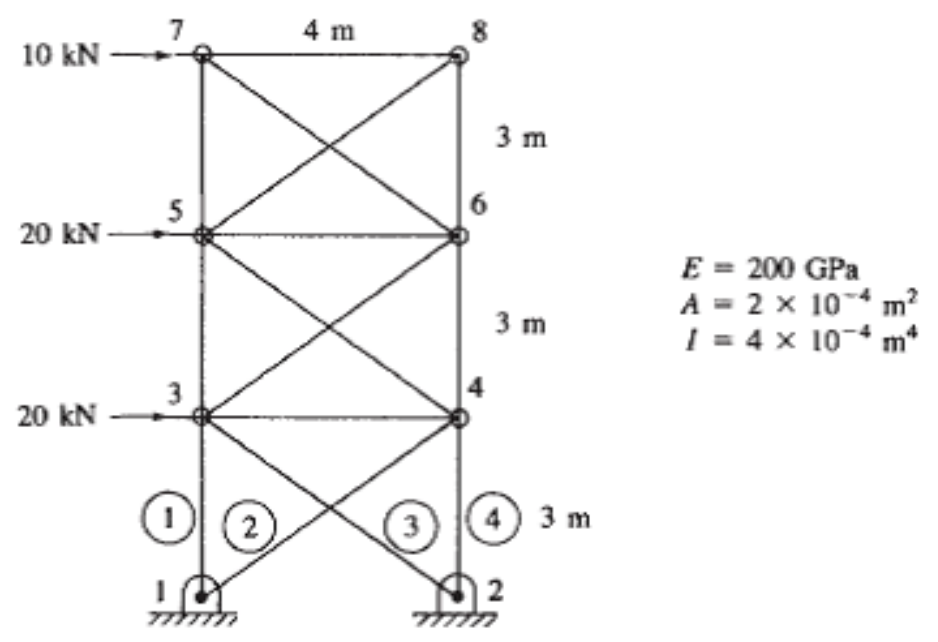

Figure B.7: Example of a plane frame with in-plane loading. ${ }^{85}$

While the full derivation will not be presented here, it can be shown that the general global stiffness matrix $[k]$ for a beam element with axial loading, shear, and bending can be written as (note $: c=\cos \theta, s=\sin \theta)$

$[k]=\frac{E}{L}\left[\begin{array}{cccccc}A c^{2}+\frac{12 I}{L^{2}} s^{2} & \left(A-\frac{12 I}{L^{2}}\right) c s & -\frac{6 I}{L} s & -\left(A c^{2}+\frac{12 I}{L^{2}} s^{2}\right) & -\left(A-\frac{12 I}{L^{2}}\right) c s & -\frac{6 I}{L} s \\ & A s^{2}+\frac{12 I}{L^{2}} c^{2} & \frac{6 I}{L} c & -\left(A-\frac{12 I}{L^{2}}\right) c s & -\left(A s^{2}+\frac{12 I}{L^{2}} c^{2}\right) & \frac{6 I}{L} c \\ & & 4 I & \frac{6 I}{L} s & -\frac{6 I}{L} c & 2 I \\ & & & A c^{2}+\frac{12 I}{L^{2}} s^{2} & \left(A-\frac{12 I}{L^{2}}\right) c s & \frac{6 I}{L} s \\ & & & A s^{2}+\frac{12 I}{L^{2}} c^{2} & -\frac{6 I}{L} c \\ \text { Symmetry } & & & & 4 I\end{array}\right]$

with elastic modulus $E$, length $L$, cross-section area $A$, and area moment of inertia $I$. It is evident that the analysis of frame systems can be computationally exhausting with an increased number of elements, and it is best to make use of computer programs to help carry out such computations. 
Unlike plane frames, grids withstand loads that are perpendicular to the grid plane (see Figure B.8), where only the transverse displacement $v$, rotation $\phi_{x}$, and rotation $\phi_{z}$ at the beam element nodes will be considered for this case (revert to Figure B.6).

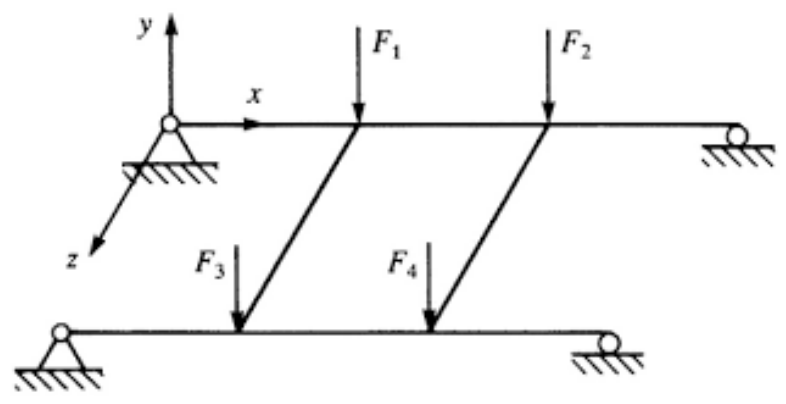

Figure B.8: Example of a grid structure with loading perpendicular to grid plane. ${ }^{85}$

While the full derivation will not be presented here, it can be shown that the local stiffness matrix $\left[k_{G}^{\prime}\right]$ for a grid element can be written as

$$
\left[k_{G}^{\prime}\right]=\left[\begin{array}{cccccc}
v_{1}^{\prime} & \phi_{1 x}^{\prime} & \phi_{1 z}^{\prime} & v_{2}^{\prime} & \phi_{2 x}^{\prime} & \phi_{2 z}^{\prime} \\
\frac{12 E I}{L^{3}} & 0 & \frac{6 E I}{L^{2}} & -\frac{12 E I}{L^{3}} & 0 & \frac{6 E I}{L^{2}} \\
0 & \frac{G J}{L} & 0 & 0 & -\frac{G J}{L} & 0 \\
\frac{6 E I}{L^{2}} & 0 & \frac{4 E I}{L} & -\frac{6 E I}{L^{2}} & 0 & \frac{2 E I}{L} \\
-\frac{12 E I}{L^{3}} & 0 & -\frac{6 E I}{L^{2}} & \frac{12 E I}{L^{3}} & 0 & -\frac{6 E I}{L^{2}} \\
0 & -\frac{G J}{L} & 0 & 0 & \frac{G J}{L} & 0 \\
\frac{6 E I}{L^{2}} & 0 & \frac{2 E I}{L} & -\frac{6 E I}{L^{2}} & 0 & \frac{4 E I}{L}
\end{array}\right]
$$

with elastic modulus $E$, length $L$, area moment of inertia $I$, shear modulus $G$, and polar area moment of inertia $J$. Like frame systems, it is best to make use of computer programs to run the calculations. The time it takes for software to reach a solution for frame and grid systems will depend on the size of the model, the number of elements, and the processing power of the computer. 


\section{B.3 Plane Stress and Plane Strain}

Spring, bar, and beam elements can be used to model systems in 2D planes or even 3D space. For these elements, a single coordinate in a given direction can describe the position of the element, and as such, these types of elements are known as line elements. ${ }^{85}$ However, there are other types of elements that require three or more nodes to define their position in a 2D plane or 3D space (see Figure B.9), and although their derivations are more complex than line elements, these planar and solid elements can provide more accurate approximations to the solution of a model or system.

\section{1-D (Line) Element}

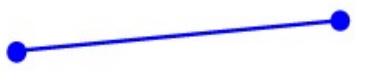

(Spring, truss, beam, pipe, etc.)

\section{2-D (Plane) Element}

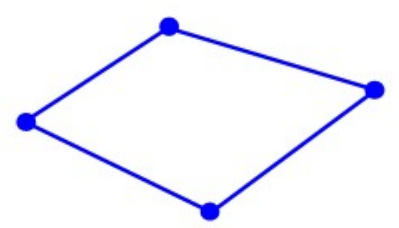

(Membrane, plate, shell, etc.)

\section{3-D (Solid) Element}

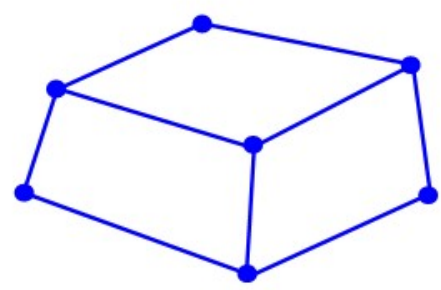

(3-D fields - temperature, displacement, stress, flow velocity)

Figure B.9: Examples of different element types used in FEM. ${ }^{45}$ 
With 2D planar elements, it is critical to recognize the importance of plane stress and plane strain in a model or system (see Figure B.10), as this will help simplify the analysis while still achieving results that are representative of the real system.

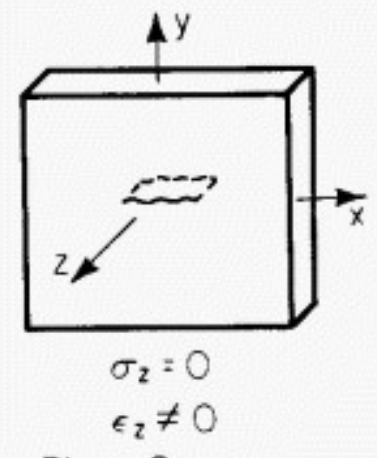

Plane Stress (Thin Body)

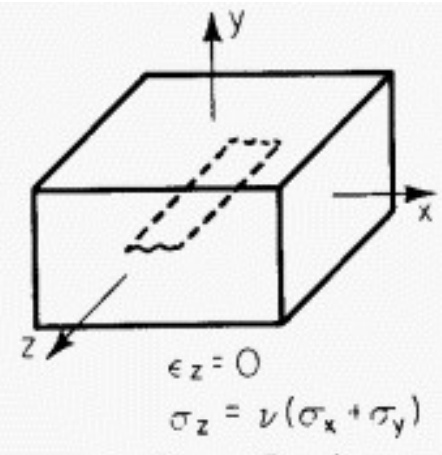

Plane Strain (Thick Body)

Figure B.10: Assumptions of plane stress (left) and plane strain (right). ${ }^{65}$

From the 2D stress state (see Figure B.11),

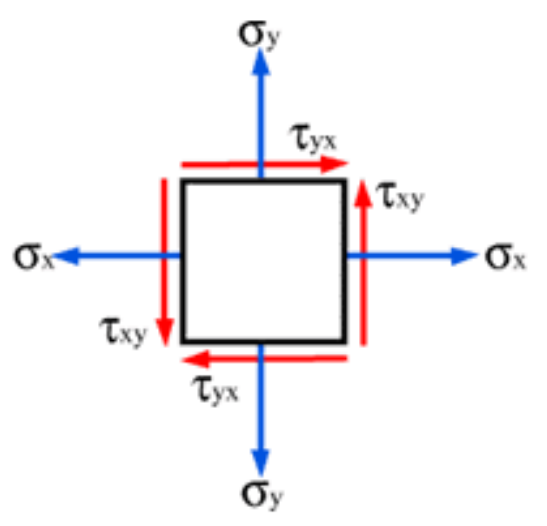

Figure B.11: 2D stress state. ${ }^{71}$

the stresses can be represented in vector form by

$$
\{\sigma\}=\left\{\begin{array}{c}
\sigma_{x} \\
\sigma_{y} \\
\tau_{x y}
\end{array}\right\}
$$


where $\sigma$ represents the normal stress and $\tau$ represents the shear stress of the state. Similarly, the strains can be represented in vector form by

$$
\{\varepsilon\}=\left\{\begin{array}{c}
\varepsilon_{x} \\
\varepsilon_{y} \\
\gamma_{x y}
\end{array}\right\}
$$

where $\varepsilon$ is the normal strain and $\gamma$ is the shear strain of the state. Also, the stressstrain relationship is defined by

$$
\{\sigma\}=[D]\{\varepsilon\}
$$

where $D$ is the constitutive matrix of the equation for isotropic materials.

In the case of plane stress where it is assumed

$$
\sigma_{z}=\tau_{x z}=\tau_{y z}=\gamma_{x z}=\gamma_{y z}=0 \quad\left(\text { note } \varepsilon_{z} \neq 0\right)
$$

it can be shown that the constitutive matrix $D$ reduces to

$$
[D]=\frac{E}{1-\nu^{2}}\left[\begin{array}{ccc}
1 & \nu & 0 \\
\nu & 1 & 0 \\
0 & 0 & \frac{1-\nu}{2}
\end{array}\right]
$$

where $E$ is the elastic modulus and $\nu$ is the Poisson's ratio of the material. In the case of plane strain where it is assumed

$$
\varepsilon_{z}=\gamma_{x z}=\gamma_{y z}=\tau_{x z}=\tau_{y z}=0 \quad\left(\text { note } \sigma_{z} \neq 0\right)
$$

it can be shown that the constitutive matrix $D$ reduces to

$$
[D]=\frac{E}{(1+\nu)(1-2 \nu)}\left[\begin{array}{ccc}
1-\nu & \nu & 0 \\
\nu & 1-\nu & 0 \\
0 & 0 & \frac{1-2 \nu}{2}
\end{array}\right]
$$

Using the plane stress or plane strain approach, it becomes evident that the constitutive matrix $D$ is simplified, thus reducing the number of subsequent calculations of a solution. Also, note the term $1-2 \nu$ in the constitutive $D$ matrix for plane strain, as it is given special attention in the discussion related to shear locking (see Appendix B.3.3 for more details). With plane stress and plane strain defined, it is possible to now develop triangular plane elements. 


\section{B.3.1 Constant Strain Triangles}

The first planar element to be discussed is the constant strain triangle (CST). This type of element requires three nodes to define its location in a $2 \mathrm{D}$ plane, where each node is defined by two coordinates (see Figure B.12), compared to one coordinate for line elements. Benefits of using CST's in FEM include their ability to model irregular-shape bodies, and a formulation that is relatively easy to apply as opposed to other types of planar elements.
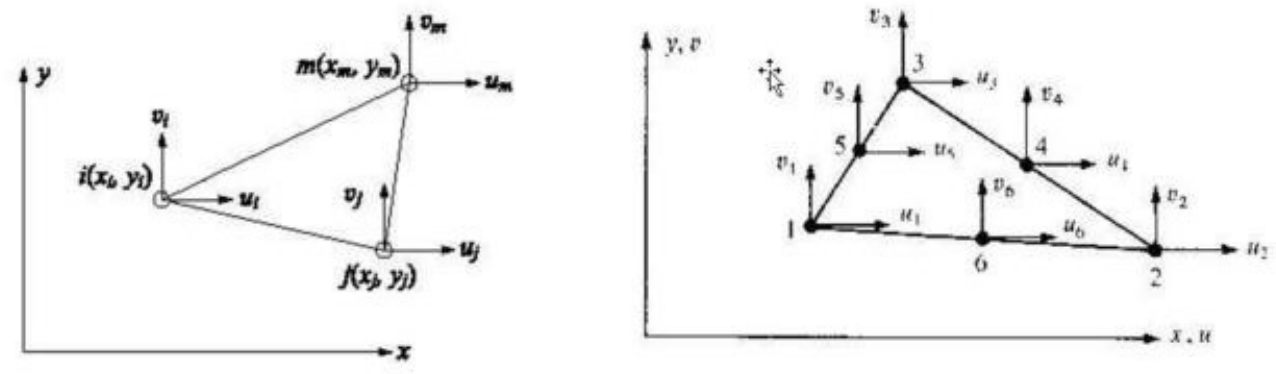

Figure B.12: Constant strain triangle (left) vs. Linear strain triangle (right). ${ }^{24}$

In order to develop the stiffness matrix for a CST element, the linear displacement functions

$$
\begin{aligned}
& u(x, y)=a_{1}+a_{2} x+a_{3} y \\
& v(x, y)=a_{4}+a_{5} x+a_{6} y
\end{aligned}
$$

are first used to define the nodal displacements of a CST. Substituting the nodal coordinates into the linear displacement functions and applying the method of cofactors, it can be shown that

$$
\begin{aligned}
& N_{i}=\frac{1}{2 A}\left(\alpha_{i}+\beta_{i} x+\gamma_{i} y\right) \\
& N_{j}=\frac{1}{2 A}\left(\alpha_{j}+\beta_{j} x+\gamma_{j} y\right) \\
& N_{m}=\frac{1}{2 A}\left(\alpha_{m}+\beta_{m} x+\gamma_{m} y\right)
\end{aligned}
$$


where $N_{i, j, m}$ are the shape functions of the CST element and

$$
\begin{array}{lll}
\alpha_{i}=x_{j} y_{m}-y_{j} x_{m} & \alpha_{j}=y_{i} x_{m}-x_{i} y_{m} & \alpha_{m}=x_{i} y_{j}-y_{i} x_{j} \\
\beta_{i}=y_{j}-y_{m} & \beta_{j}=y_{m}-y_{i} & \beta_{m}=y_{i}-y_{j} \\
\gamma_{i}=x_{m}-x_{j} & \gamma_{j}=x_{i}-x_{m} & \gamma_{m}=x_{j}-x_{i}
\end{array}
$$

as noted in Figure B.12. Using the strain-displacement relationship

$$
\{\varepsilon\}=[B]\{d\}
$$

the gradient matrix $[B]$ depends only on the element nodal coordinates (independent of $\mathrm{x}-\mathrm{y}$ coordinates) and

$$
[B]=\left[\begin{array}{lll}
{\left[B_{i}\right]} & {\left[B_{j}\right]} & {\left[B_{m}\right.}
\end{array}\right]
$$

where

$$
\left[B_{i}\right]=\frac{1}{2 A}\left[\begin{array}{cc}
\beta_{i} & 0 \\
0 & \gamma_{i} \\
\gamma_{i} & \beta_{i}
\end{array}\right] \quad\left[B_{j}\right]=\frac{1}{2 A}\left[\begin{array}{cc}
\beta_{j} & 0 \\
0 & \gamma_{j} \\
\gamma_{j} & \beta_{j}
\end{array}\right] \quad\left[B_{m}\right]=\frac{1}{2 A}\left[\begin{array}{cc}
\beta_{m} & 0 \\
0 & \gamma_{m} \\
\gamma_{m} & \beta_{m}
\end{array}\right]
$$

For plane stress problems, Equation B.31 can be written as

$$
\{\sigma\}=[D][B]\{d\}
$$

where $[D]$ is the constitutive matrix and both stress and strain are assumed to be constant throughout the element. ${ }^{85}$ Finally, by applying the potential energy method to the CST element, it can be shown that

$$
[k]=t A[B]^{T}[D][B]
$$

where $[k]$ is the stiffness matrix for a CST element, $A$ is the area of the triangle, and $t$ is the thickness of the element. While this discussion was limited to CST elements, linear strain triangle (LST) elements can also be used for 2D analysis, as they allow for better control of the elements due to the three additional nodes in their definition. CST and LST each have their own set of benefits, and although LST elements are more complex, they are derived using the same principles presented here. 


\section{B.3.2 Rectangular Plane Element}

Rectangular plane elements can also be used to analyze 2D models or systems (see Figure B.13). Unlike constant strain triangle (CST) elements, rectangular plane elements use four corner nodes to define the element. Benefits of using rectangular plane elements over CST elements include simpler input of data and interpretation of stresses ${ }^{85}$ However, rectangular plane elements have the drawback of poorly representing boundary conditions due to their straight sides, especially for geometries with irregular shapes.

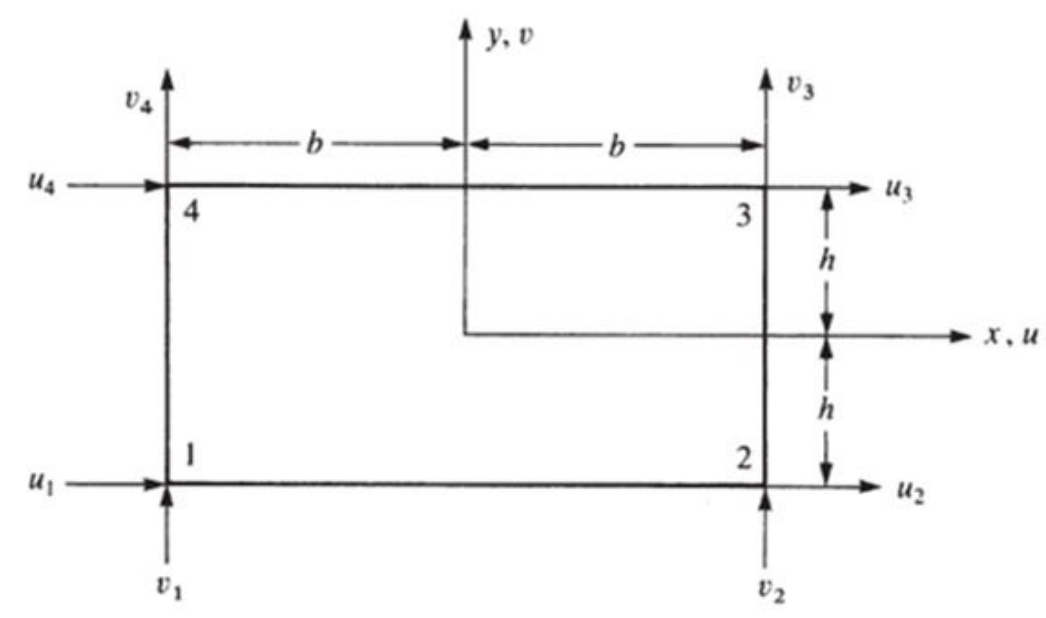

Figure B.13: Simple rectangular plane element. ${ }^{32}$

To develop the stiffness matrix for a rectangular plane element, the linear displacement functions

$$
\begin{aligned}
& u(x, y)=a_{1}+a_{2} x+a_{3} y+a_{4} x y \\
& v(x, y)=a_{5}+a_{6} x+a_{7} y+a_{8} x y
\end{aligned}
$$

are first used to define the nodal displacements of the rectangular plane element. Substituting the nodal coordinates into the linear displacement functions and applying 
the method of co-factors, it can be shown that

$$
\begin{array}{ll}
N_{1}=\frac{(b-x)(h-y)}{4 b h} & N_{2}=\frac{(b+x)(h-y)}{4 b h} \\
N_{3}=\frac{(b+x)(h+y)}{4 b h} & N_{4}=\frac{(b-x)(h+y)}{4 b h}
\end{array}
$$

where $N_{1,2,3,4}$ are the shape functions of the rectangular plane element. Using the strain-displacement relationship

$$
\{\varepsilon\}=[B]\{d\}
$$

the gradient matrix $[B]$ depends only on the element nodal coordinates (independent of $\mathrm{x}-\mathrm{y}$ coordinates) and

$$
[B]=\frac{1}{4 b h}\left[\begin{array}{ccccc}
-(h-y) & 0 & (h-y) & 0 & \\
0 & -(b-x) & 0 & -(b+x) & \\
-(b-x) & -(h-y) & -(b+x) & (h-y) & \\
& (h+y) & 0 & -(h+y) & 0 \\
0 & (b+x) & 0 & (b-x) \\
(b+x) & (h+y) & (b-x) & -(h+y)
\end{array}\right]
$$

Finally, by applying the potential energy method to the rectangular plane element, it can be shown that

$$
[k]=\int_{-h}^{h} \int_{-b}^{b}[B]^{T}[D][B] t d x d y
$$

where $[k]$ is the stiffness matrix for a rectangular plane element, $h$ is the height dimension, $b$ is the base dimension, $[D]$ is the constitutive matrix, and $t$ is the thickness of the element.

While this discussion was limited to simple rectangular plane elements, quadratic (8 nodes) and cubic (12 nodes) rectangular elements can also be used for a 2D analysis, as they allow for better control of the elements in the model. Simple, quadratic, and cubic rectangular elements each have their own set of benefits, and sound engineering judgment should be used to select the element best suited for the model and analysis being performed. 


\section{B.3.3 Shear Locking}

Recall from classic beam theory that for elements in a state of pure bending, the shear stress, and subsequently shear strain, of the element are assumed to be zero. However, because constant strain triangles (CST) and rectangular plane elements are used as linear approximations of a model or system, the straight sides of these elements poorly imitate the real behavior of edges in pure bending (see Figure B.14). As a result, pseudo shear stresses and strains appear in the linear elements, which take energy away from the bending moment and thus making the element "stiffer" than what it should be. ${ }^{85}$ This phenomena in linear elements is known as shear locking.

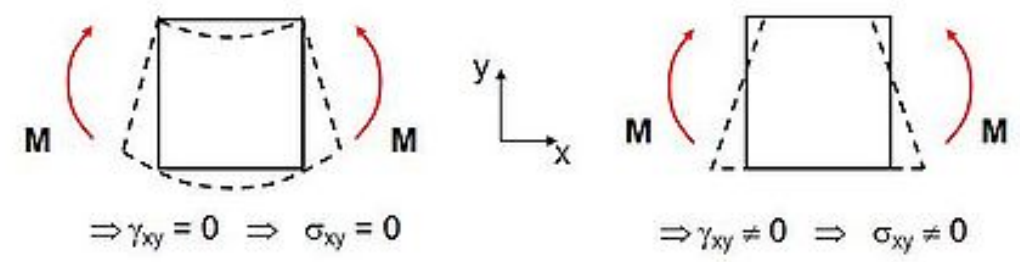

Figure B.14: Exact bending deformation (left) vs linear element deformation (right). ${ }^{62}$

In a pure bending state, the deformations lines of an element are, and remain, orthogonal to each other even after bending. However, linear elements have deformation lines that can't exactly model bending due to the linear behavior of the element, and as a result lose orthogonality with an applied bending moment (see Figure B.15).

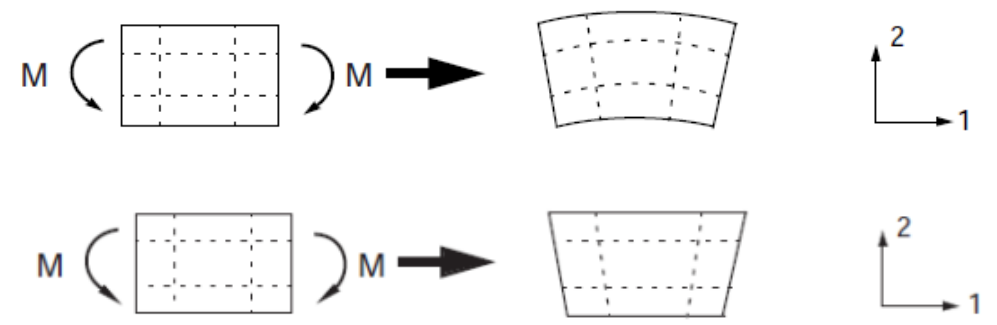

Figure B.15: True deformation lines at $90^{\circ}$ with respect to each other (top), as opposed to deformation lines of a linear element (bottom). ${ }^{82}$ 
For a more mathematical observation, recall from the discussion on plane strain (see Appendix B.3 for more details) that

$$
[D]=\frac{E}{(1+\nu)(1-2 \nu)}\left[\begin{array}{ccc}
1-\nu & \nu & 0 \\
\nu & 1-\nu & 0 \\
0 & 0 & \frac{1-2 \nu}{2}
\end{array}\right]
$$

where $[D]$ is the constitutive matrix for linear elements in plane strain, $E$ is the elastic modulus, and $\nu$ is Poisson's ratio of the material. In particular, the term $1-2 \nu$ deserves special attention here. The shear locking phenomena is not limited to the poor representation of bending in linear elements, as the material used in the analysis can also affect the severity of locking in a model. Note that as Poisson's ratio $\nu$ approaches 0.5 in Equation B.41, the denominator becomes small, which adversely increases the magnitude of the constitutive matrix [D]. When applied to the stiffness matrix of a CST element for example,

$$
[k]=t A[B]^{T}[D][B]
$$

it becomes evident that the stiffness $[k]$ of the element will also increase significantly, to the point where a computer program can "lock" as it attempts to complete the element calculations. This is known as volumetric locking and is a result of the exaggerated stiffness of the element, where the software now considers the material nearly incompressible, with little to no degrees of freedom available to solve in the model or system. ${ }^{85}$ This is one of the main reasons why it is difficult to model materials like rubber, which has a Poisson's ratio that ranges from 0.48 to $0.5^{59}$ in most cases.

Engineers should proceed with caution when analyzing such models, as the "exaggerated" stiffness may lead to overdesign of the model or system. For the analysis of elastic materials like rubber, it is necessary to apply additional techniques in the computer model setup in order to minimize the effects of shear and volumetric locking. 


\section{B.4 3D Stress Analysis}

The focus now shifts to defining 3D solid elements. These elements are well suited for analyzing 3D models, especially when it comes to extracting stress information from the model. Although the derivations of 3D solid elements are significantly more involved than 2D planar elements, both types of elements are developed using similar principles. Due to the additional number of nodes required to define solid elements, these type of elements can provide more accurate results, but increase the computation time needed to reach a solution. There are several types of 3D solid elements that can be applied in FEM (see Figure B.16), but this section will focus on simple tetrahedron and hexahedron "brick" elements, which are commonly used in 3D stress analysis.

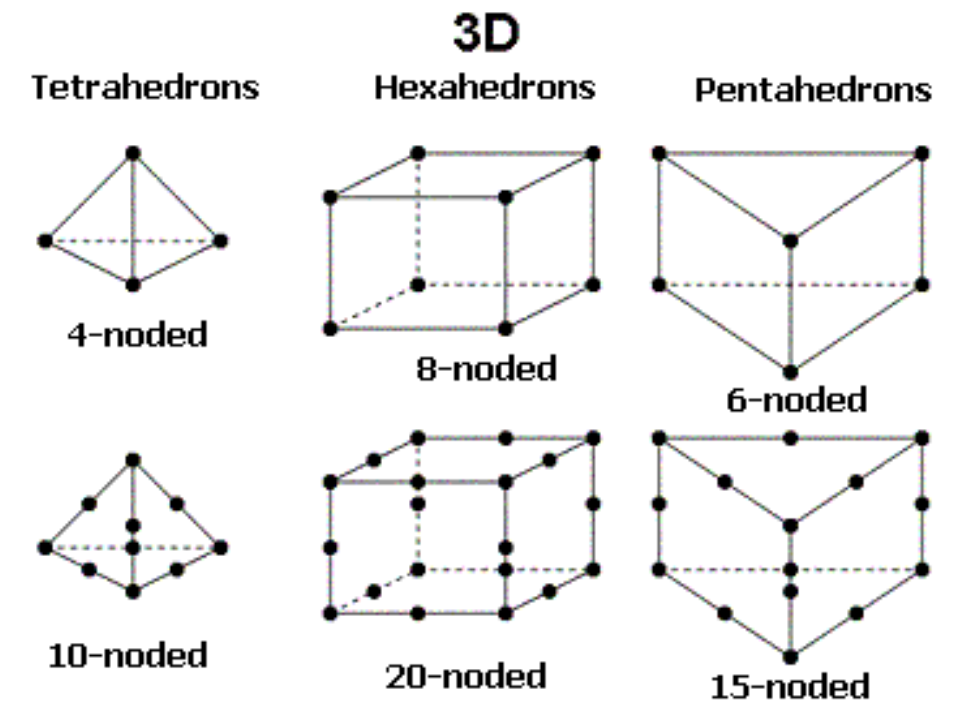

Figure B.16: Example of various 3D element shapes. ${ }^{56}$ 
Consider the 3D stress element (see Figure B.17)

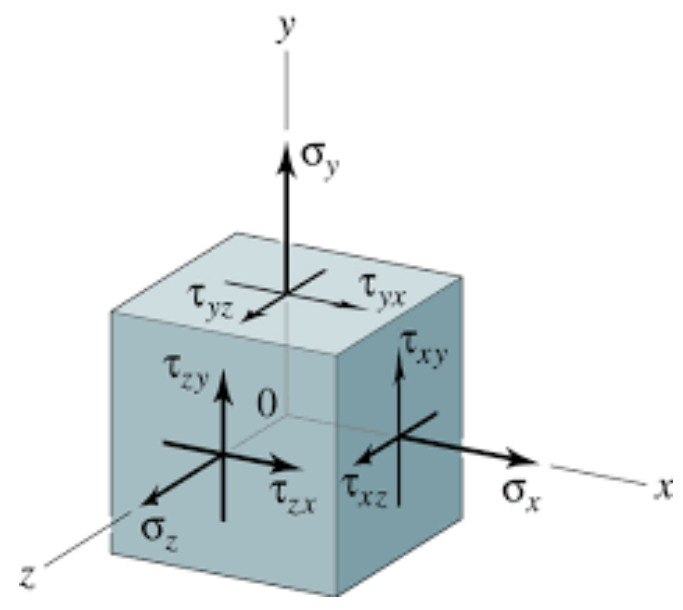

Figure B.17: $3 \mathrm{D}$ stress element. ${ }^{1}$

where

$$
\{\sigma\}=\left\{\begin{array}{c}
\sigma_{x} \\
\sigma_{y} \\
\sigma_{z} \\
\tau_{x y} \\
\tau_{y z} \\
\tau_{z x}
\end{array}\right\} \quad\{\varepsilon\}=\left\{\begin{array}{c}
\varepsilon_{x} \\
\varepsilon_{y} \\
\varepsilon_{z} \\
\gamma_{x y} \\
\gamma_{y z} \\
\gamma_{z x}
\end{array}\right\}
$$

are the stress $\{\sigma\}$ and strain $\{\varepsilon\}$ states of the 3D element. Applying the stress-strain relationship

$$
\{\sigma\}=[D]\{\varepsilon\}
$$

and combining with the shear strain expressions of the solid element, it can be shown that

$$
[D]=\frac{E}{E}\left[\begin{array}{cccccc}
1-\nu & \nu & \nu & 0 & 0 & 0 \\
& 1-\nu & \nu & 0 & 0 & 0 \\
& & 1-\nu & 0 & 0 & 0 \\
& & & \frac{1-2 \nu}{2} & 0 & 0 \\
\text { Symmetry } & & & & \frac{1-2 \nu}{2} & 0 \\
& & & & & \\
& & & &
\end{array}\right.
$$

where $[D]$ is the constitutive matrix of a 3D solid element, $E$ is the elastic modulus, and $\nu$ is Poisson's ratio of the material. 
For tetrahedrals, the linear displacement functions

$$
\begin{aligned}
& u(x, y, z)=a_{1}+a_{2} x+a_{3} y+a_{4} z \\
& v(x, y, z)=a_{5}+a_{6} x+a_{7} y+a_{8} z \\
& w(x, y, z)=a_{9}+a_{10} x+a_{11} y+a_{12} z
\end{aligned}
$$

are used to define the nodal displacements of the tetrahedral element. Substituting the nodal coordinates into the linear displacement functions and solving, it can be shown that

$$
\begin{array}{ll}
N_{1}=\frac{\alpha_{1}+\beta_{1} x+\gamma_{1} y+\delta_{1} z}{6 V} & N_{2}=\frac{\alpha_{2}+\beta_{2} x+\gamma_{2} y+\delta_{2} z}{6 V} \\
N_{3}=\frac{\alpha_{3}+\beta_{3} x+\gamma_{3} y+\delta_{3} z}{6 V} & N_{4}=\frac{\alpha_{4}+\beta_{4} x+\gamma_{4} y+\delta_{4} z}{6 V}
\end{array}
$$

where $N_{1,2,3,4}$ are the shape functions of the solid tetrahedral element and

$$
6 V=\left|\begin{array}{cccc}
1 & x_{1} & y_{1} & z_{1} \\
1 & x_{2} & y_{2} & z_{2} \\
1 & x_{3} & y_{3} & z_{3} \\
1 & x_{4} & y_{4} & z_{4}
\end{array}\right|
$$

with nodal coordinates $x_{i}, y_{i}$, and $z_{i}$. Using the strain-displacement relationship

$$
\{\varepsilon\}=[B]\{d\}
$$

the gradient matrix $[B]$ depends only on the element nodal coordinates (independent of $\mathrm{x}-\mathrm{y}-\mathrm{z}$ coordinates) and

$$
[B]=\left[\begin{array}{llll}
{\left[B_{1}\right]} & {\left[B_{2}\right]} & {\left[B_{3}\right]} & {\left[B_{4}\right]}
\end{array}\right]
$$

where

$$
\left[B_{1}\right]=\frac{1}{6 V}\left[\begin{array}{ccc}
\beta_{1} & 0 & 0 \\
0 & \gamma_{1} & 0 \\
0 & 0 & \delta_{1} \\
\gamma_{1} & \beta_{1} & 0 \\
0 & \delta_{1} & \gamma_{1} \\
\delta_{1} & 0 & \beta_{1}
\end{array}\right]
$$

Similarly, $\left[B_{2}\right],\left[B_{3}\right]$, and $\left[B_{4}\right]$ can be found by substituting the corresponding coefficients $\alpha_{i}, \beta_{i}, \gamma_{i}$, and $\delta i$ (refer to p. 539 of text ${ }^{85}$ for coefficient definitions). 
Applying the stress-strain relationship for isotropic materials (Equation B.44), it can be shown that

$$
[k]=[B]^{T}[D][B] V
$$

where $[\mathrm{k}]$ is the $12 \times 12$ stiffness matrix for a simple tetrahedral element, and $V$ is the volume of the tetrahedral element.

For hexahedron brick elements with natural coordinates $s-t-z^{\prime}$, the element geometry is defined by

$$
x=a_{1}+a_{2} s+a_{3} t+a_{4} z^{\prime}+a_{5} s t+a_{6} t z^{\prime}+a_{7} z^{\prime} s+a_{8} s t z^{\prime}
$$

where

$$
N_{i}=\frac{\left(1+s s_{i}\right)\left(1+t t_{i}\right)\left(1+z^{\prime} z_{i}^{\prime}\right)}{8}
$$

represents the eight corresponding shape functions of the hexahedron element. Applying the displacement function of the element (x-direction)

$$
u=a_{1}+a_{2} s+a_{3} t+a_{4} z^{\prime}+a_{5} s t+a_{6} t z^{\prime}+a_{7} z^{\prime} s+a_{8} s t z^{\prime}
$$

and expressing the strains in terms of the shape functions and global coordinates, it can be shown that

$$
[k]=\int_{-1}^{1} \int_{-1}^{1} \int_{-1}^{1}[B]^{T}[D][B]|[J]| d s d t d z^{\prime}
$$

where $[k]$ is the $24 \times 24$ stiffness matrix of a hexahedron brick element, and $[J]$ is the Jacobian matrix which relates the element area in the global coordinate system to the element area in the natural coordinate system. Like rectangular plane elements, linear brick elements also do a poor job of modeling bending, and as such, fall victim to shear locking (see Appendix B.3.3 for more details). However, solid elements like the 20-noded quadratic hexahedron element (revert to Figure B.16) can be used instead to remedy the effects of shear locking. 


\section{B.5 Plate Bending Elements}

Having discussed 3D solid elements for thick bodies, plate elements will now be used to analyze thin plate structures. Plate elements are one of the most important FEM tools in the field of engineering, as they can be used to model many critical systems like vehicle bodies and aircraft structures. For these derivations, the classical thinplate theory will be applied, where the thickness of the plate is significantly smaller than the other plate dimensions, and the deflection $w$ is significantly smaller than the thickness of the plate ${ }^{85}$ (see Figure B.18) .

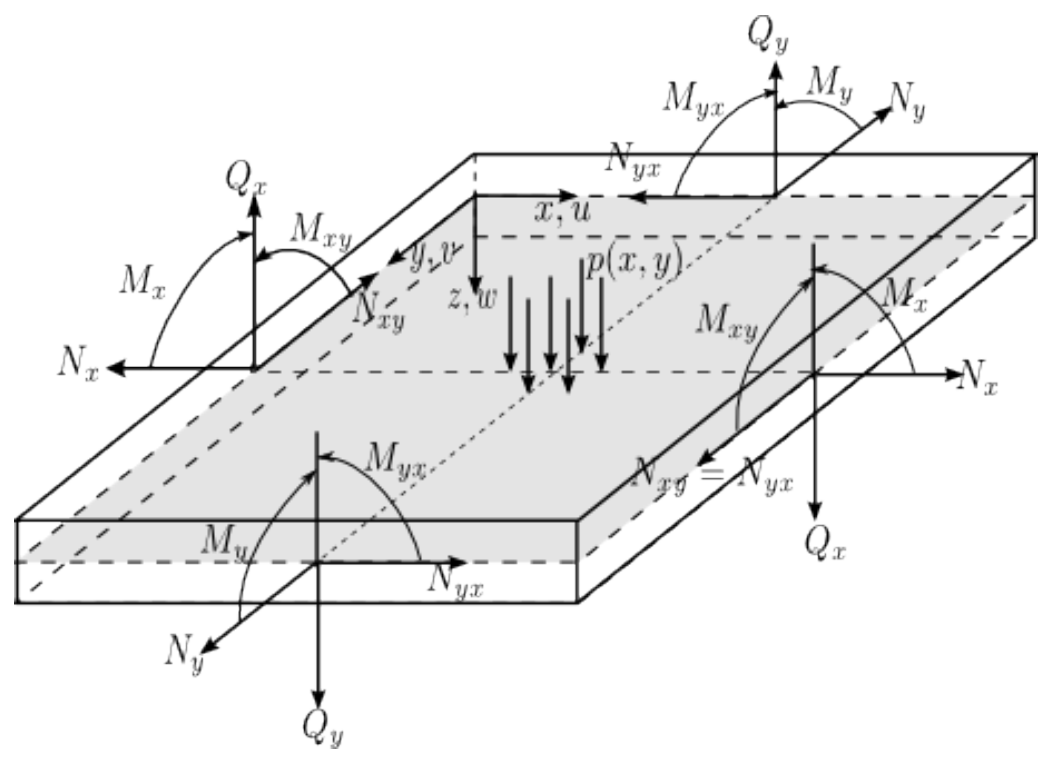

Figure B.18: Plate element with differential moments and forces (stresses on edges not shown). ${ }^{78}$

The curvature $\kappa$ of the plate is first defined as the rate of change of the normal angular displacement where

$$
\kappa_{x}=-\frac{\partial^{2} w}{\partial x^{2}} \quad \kappa_{y}=-\frac{\partial^{2} w}{\partial y^{2}} \quad \kappa_{x y}=-\frac{2 \partial^{2} w}{\partial x \partial y}
$$


and applying the in-plane strain definitions of a $2 \mathrm{D}$ stress element, the strain $\varepsilon$ equations for a plate element can be written as

$$
\varepsilon_{x}=-z \frac{\partial^{2} w}{\partial x^{2}} \quad \varepsilon_{y}=-z \frac{\partial^{2} w}{\partial y^{2}} \quad \gamma_{x y}=-2 z \frac{\partial^{2} w}{\partial x \partial y}
$$

Recall that for an isotropic material in plane stress,

$$
\begin{aligned}
\sigma_{x} & =\frac{E}{1-\nu^{2}}\left(\varepsilon_{x}-\nu \varepsilon_{y}\right) \\
\sigma_{y} & =\frac{E}{1-\nu^{2}}\left(\varepsilon_{y}-\nu \varepsilon_{x}\right) \\
\tau_{x y} & =G \gamma_{x y}
\end{aligned}
$$

where $E$ is the elastic modulus, and $G$ is the modulus of rigidity for the material. Using the differential element of a plate, it can be shown that

$$
M_{x}=D\left(\kappa_{x}+\nu \kappa_{y}\right) \quad M_{y}=D\left(\kappa_{y}+\nu \kappa_{x}\right) \quad M_{x y}=\frac{D(1-\nu)}{2} \kappa_{x y}
$$

where $M$ is the moment-curvature relation for a plate element and

$$
D=\frac{E t^{3}}{12\left(1-\nu^{2}\right)}
$$

is the bending rigidity of the plate with thickness $t$. Letting

$$
\{\kappa\}=[Q]\{a\}
$$

the curvature matrix $\{\kappa\}$ can be expressed as

$$
\{\kappa\}=[B]\{d\}
$$

where

$$
[B]=[Q][C]^{-1}
$$


The moment-curvature matrix $\{M\}$ can then be re-written as

$$
\{M\}=\left\{\begin{array}{c}
M_{x} \\
M_{y} \\
M_{x y}
\end{array}\right\}=[D]\left\{\begin{array}{c}
\kappa_{x} \\
\kappa_{y} \\
\kappa_{x y}
\end{array}\right\}=[D][B]\{d\}
$$

where

$$
[D]=\frac{E t^{3}}{12\left(1-\nu^{2}\right)}\left[\begin{array}{ccc}
1 & \nu & 0 \\
\nu & 1 & 0 \\
0 & 0 & \frac{1-\nu}{2}
\end{array}\right]
$$

is the constitutive matrix for the plate element. Combining terms,

$$
[k]=\iint[B]^{T}[D][B] d x d y
$$

where $[k]$ is the $12 \times 12$ stiffness matrix and $[B]$ is the gradient matrix for a four-noded isotropic plate element.

Although a four-noded plate element was discussed here, there are other types of plate elements available for FEM analysis, including a solution derived from a 16term displacement function polynomial, ${ }^{85}$ as opposed to the 12 -term polynomial used in the background derivations of this section. Each type of plate element has its own set of benefits, and should be selected based on the plate model and type of analysis being performed. 
Appendix C

PCB ACCELEROMETER USER MANUAL

[See Attachment] 
Model 356A03

Triaxial ICP $\circledast$ Accelerometer

Installation and Operating Manual

For assistance with the operation of this product, contact PCB Piezotronics, Inc.

Toll-free: 800-828-8840

24-hour SensorLine: 716-684-0001

Fax: 716-684-0987

E-mail: info@pcb.com

Web: www.pcb.com

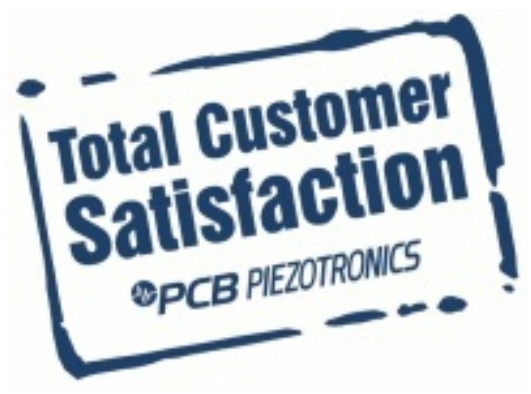




\section{DPCB PIEZOTRONICS}

Service, Repair, and Return

Policies and Instructions

The information contained in this document supersedes all similar information that may be found elsewhere in this manual.

Service - Due to the sophisticated nature of the sensors and associated instrumentation provided by PCB Piezotronics, user servicing or repair is not recommended and, if attempted, may void the factory warranty. Routine maintenance, such as the cleaning of electrical connectors, housings, and mounting surfaces with solutions and techniques that will not harm the physical material of construction, is acceptable. Caution should be observed to ensure that liquids are not permitted to migrate into devices that are not hermetically sealed. Such devices should only be wiped with a dampened cloth and never submerged or have liquids poured upon them.

Repair - In the event that equipment becomes damaged or ceases to operate, arrangements should be made to return the equipment to $\mathrm{PCB}$ Piezotronics for repair. User servicing or repair is not recommended and, if attempted, may void the factory warranty.

Calibration - Routine calibration of sensors and associated instrumentation is recommended as this helps build confidence in measurement accuracy and acquired data. Equipment calibration cycles are typically established by the users own quality regimen. When in doubt about a calibration cycle, a good "rule of thumb" is to recalibrate on an annual basis. It is also good practice to recalibrate after exposure to any severe temperature extreme, shock, load, or other environmental influence, or prior to any critical test.

PCB Piezotronics maintains an ISO9001 certified metrology laboratory and offers calibration services, which are accredited by A2LA to ISO/IEC 17025, with full traceability to $\mathrm{SI}$ through N.I.S.T. In addition to the normally supplied calibration, special testing is also available, such as: sensitivity at elevated or cryogenic temperatures, phase response, extended high or low frequency response, extended range, leak testing, hydrostatic pressure testing, and others. For information on standard recalibration services or special testing, contact your local PCB Piezotronics distributor, sales representative, or factory customer service representative.

Returning Equipment - Following these procedures will ensure that your returned materials are handled in the most expedient manner. Before returning any equipment to $\mathrm{PCB}$ Piezotronics, contact your local distributor, sales representative, or factory customer service representative to obtain a Return Warranty, Service, Repair, and Return Policies and Instructions Materials Authorization (RMA) Number. This RMA number should be clearly marked on the outside of all package(s) and on the packing 
list(s) accompanying the shipment. A detailed account of the nature of the problem(s) being experienced with the equipment should also be included inside the package(s) containing any returned materials.

A Purchase Order, included with the returned materials, will expedite the turn-around of serviced equipment. It is recommended to include authorization on the Purchase Order for PCB to proceed with any repairs, as long as they do not exceed $50 \%$ of the replacement cost of the returned item(s). PCB will provide a price quotation or replacement recommendation for any item whose repair costs would exceed $50 \%$ of replacement cost, or any item that is not economically feasible to repair. For routine calibration services, the Purchase Order should include authorization to proceed and return at current pricing, which can be obtained from a factory customer service representative.

Contact Information - International customers should direct all inquiries to their local distributor or sales office. A complete list of distributors and offices can be found at www.pcb.com. Customers within the United States may contact their local sales representative or a factory customer service representative. A complete list of sales representatives can be found at www.pcb.com. Toll-free telephone numbers for a factory customer service representative, in the division responsible for this product, can be found on the title page at the front of this manual. Our ship to address and general contact numbers are:

PCB Piezotronics, Inc.

3425 Walden Ave.

Depew, NY14043 USA

Toll-free: (800) 828-8840

24-hour SensorLine ${ }^{\text {SM: }}$ (716) 684-0001

Website: www.pcb.com

E-mail: info@pcb.com 
PCB工业监视和测量设备 - 中国RoHS2公布表

PCB Industrial Monitoring and Measuring Equipment - China RoHS 2 Disclosure Table

\begin{tabular}{|c|c|c|c|c|c|c|}
\hline \multirow[b]{2}{*}{ 部件名称 } & \multicolumn{6}{|c|}{ 有害物质 } \\
\hline & 铅 (Pb) & $\begin{array}{l}\text { 永 } \\
(\mathrm{Hg})\end{array}$ & $\begin{array}{l}\text { 镉 } \\
\text { (Cd) }\end{array}$ & 六价铭 (Cr(VI)) & 多溴联苯 (PBB) & 多溴二苯醚 (PBDE) \\
\hline 住房 & $\mathrm{O}$ & O & O & $\mathrm{O}$ & $\mathrm{O}$ & $\mathrm{O}$ \\
\hline PCB板 & $\mathrm{X}$ & $\mathrm{O}$ & $\mathrm{O}$ & $\mathrm{O}$ & 0 & $\mathrm{O}$ \\
\hline 电气连接器 & $\mathrm{O}$ & $\mathrm{O}$ & $\mathrm{O}$ & $\mathrm{O}$ & $\mathrm{O}$ & $\mathrm{O}$ \\
\hline 压电晶体 & $\mathrm{X}$ & $\mathrm{O}$ & $\mathrm{O}$ & $\mathrm{O}$ & $\mathrm{O}$ & $\mathrm{O}$ \\
\hline 环氧 & $\mathrm{O}$ & $\mathrm{O}$ & $\mathrm{O}$ & 0 & 0 & 0 \\
\hline 铁氟龙 & $\mathrm{O}$ & $\mathrm{O}$ & $\mathrm{O}$ & $\mathrm{O}$ & $\mathrm{O}$ & $\mathrm{O}$ \\
\hline 电子 & $\mathrm{O}$ & $\mathrm{O}$ & $\mathrm{O}$ & $\mathrm{O}$ & 0 & 0 \\
\hline 厚膜基板 & $\mathrm{O}$ & $\mathrm{O}$ & $\mathrm{X}$ & $\mathrm{O}$ & $\mathrm{O}$ & $\mathrm{O}$ \\
\hline 电线 & $\mathrm{O}$ & $\mathrm{O}$ & $\mathrm{O}$ & $\mathrm{O}$ & 0 & 0 \\
\hline 电缆 & $\mathrm{X}$ & $\mathrm{O}$ & $\mathrm{O}$ & $\mathrm{O}$ & $\mathrm{O}$ & $\mathrm{O}$ \\
\hline 塑料 & 0 & 0 & 0 & 0 & 0 & 0 \\
\hline 焊接 & $\mathrm{X}$ & $\mathrm{O}$ & $\mathrm{O}$ & $\mathrm{O}$ & $\mathrm{O}$ & $\mathrm{O}$ \\
\hline 铜合金/黄铜 & $\mathrm{X}$ & $\mathrm{O}$ & $\mathrm{O}$ & $\mathrm{O}$ & $\mathrm{O}$ & $\mathrm{O}$ \\
\hline \multicolumn{7}{|c|}{ 本表格依据 SJ/T 11364 的规定编制。 } \\
\hline \multicolumn{7}{|c|}{ O：表示该有害物质在该部件所有均质材料中的含量均在 GB/T 26572 规定的限量要求以下。 } \\
\hline \multicolumn{7}{|c|}{ 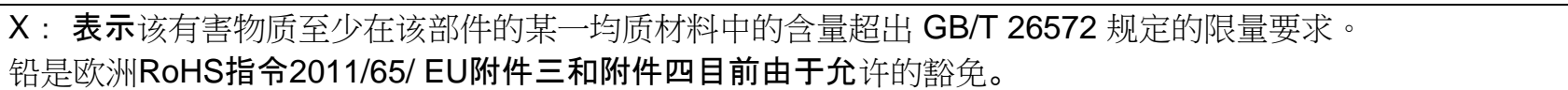 } \\
\hline
\end{tabular}




\begin{tabular}{|c|c|c|c|c|c|c|}
\hline \multirow[t]{2}{*}{ Component Name } & \multicolumn{6}{|c|}{ Hazardous Substances } \\
\hline & $\begin{array}{l}\text { Lead } \\
(\mathrm{Pb})\end{array}$ & $\begin{array}{l}\text { Mercury } \\
(\mathrm{Hg})\end{array}$ & $\begin{array}{l}\text { Cadmium } \\
\text { (Cd) }\end{array}$ & $\begin{array}{l}\text { Chromium VI } \\
\text { Compounds } \\
(\mathrm{Cr}(\mathrm{VI}))\end{array}$ & $\begin{array}{l}\text { Polybrominated } \\
\text { Biphenyls } \\
\text { (PBB) }\end{array}$ & $\begin{array}{l}\text { Polybrominated } \\
\text { Diphenyl } \\
\text { Ethers (PBDE) }\end{array}$ \\
\hline Housing & $\mathrm{O}$ & $\mathrm{O}$ & $\mathrm{O}$ & $\mathrm{O}$ & $\mathrm{O}$ & $\mathrm{O}$ \\
\hline PCB Board & $\mathrm{X}$ & $\mathrm{O}$ & $\mathrm{O}$ & $\mathrm{O}$ & $\mathrm{O}$ & $\mathrm{O}$ \\
\hline $\begin{array}{l}\text { Electrical } \\
\text { Connectors }\end{array}$ & $\mathrm{O}$ & $\mathrm{O}$ & $\mathrm{O}$ & $\mathrm{O}$ & $\mathrm{O}$ & $\mathrm{O}$ \\
\hline $\begin{array}{l}\text { Piezoelectric } \\
\text { Crystals }\end{array}$ & $\mathrm{X}$ & $\mathrm{O}$ & $\mathrm{O}$ & $\mathrm{O}$ & $\mathrm{O}$ & $\mathrm{O}$ \\
\hline Epoxy & 0 & 0 & $\mathrm{O}$ & $\mathrm{O}$ & $\mathrm{O}$ & $\mathrm{O}$ \\
\hline Teflon & $\mathrm{O}$ & $\mathrm{O}$ & $\mathrm{O}$ & $\mathrm{O}$ & $\mathrm{O}$ & $\mathrm{O}$ \\
\hline Electronics & $\mathrm{O}$ & $\mathrm{O}$ & $\mathrm{O}$ & $\mathrm{O}$ & $\mathrm{O}$ & $\mathrm{O}$ \\
\hline $\begin{array}{l}\text { Thick Film } \\
\text { Substrate }\end{array}$ & $\mathrm{O}$ & $\mathrm{O}$ & $\mathrm{X}$ & $\mathrm{O}$ & $\mathrm{O}$ & $\mathrm{O}$ \\
\hline Wires & 0 & 0 & 0 & 0 & $\mathrm{O}$ & 0 \\
\hline Cables & $\mathrm{X}$ & $\mathrm{O}$ & $\mathrm{O}$ & $\mathrm{O}$ & $\mathrm{O}$ & $\mathrm{O}$ \\
\hline Plastic & $\mathrm{O}$ & 0 & $\mathrm{O}$ & $\mathrm{O}$ & 0 & $\mathrm{O}$ \\
\hline Solder & $\mathrm{X}$ & $\mathrm{O}$ & $\mathrm{O}$ & $\mathrm{O}$ & $\mathrm{O}$ & $\mathrm{O}$ \\
\hline Copper Alloy/Brass & $X$ & $\mathrm{O}$ & $\mathrm{O}$ & $\mathrm{O}$ & $\mathrm{O}$ & $\mathrm{O}$ \\
\hline \multicolumn{7}{|c|}{ This table is prepared in accordance with the provisions of SJ/T 11364 . } \\
\hline \multicolumn{7}{|c|}{$\begin{array}{l}\text { O: Indicates that said hazardous substance contained in all of the homogeneous materials for this part is below the limit } \\
\text { requirement of GB/T } 26572 \text {. }\end{array}$} \\
\hline \multicolumn{7}{|c|}{$\begin{array}{l}\text { X: Indicates that said hazardous substance contained in at least one of the homogeneous materials for this part is above } \\
\text { the limit requirement of GB/T } 26572 \text {. } \\
\text { Lead is present due to allowed exemption in Annex III or Annex IV of the European RoHS Directive 2011/65/EU. }\end{array}$} \\
\hline
\end{tabular}




\title{
General \\ OPERATING GUIDE
}

for use with

\section{PIEZOELECTRIC ICP ${ }^{\circledR}$ ACCELEROMETERS}

\author{
SPECIFICATION SHEET, INSTALLATION DRAWING \\ AND CALIBRATION INFORMATION ENCLOSED
}

\begin{abstract}
PCB ASSUMES NO RESPONSIBILITY FOR DAMAGE CAUSED TO THIS PRODUCT AS A RESULT OF PROCEDURES THAT ARE INCONSISTENT WITH THIS OPERATING GUIDE.
\end{abstract}

\subsection{INTRODUCTION}

Congratulations on the purchase of a quality, ICP ${ }^{\circledR}$ acceleration sensor. In order to ensure the highest level of performance for this product, it is imperative that you properly familiarize yourself with the correct mounting and installation techniques before attempting to operate this device. If, after reading this manual, you have any additional questions concerning this sensor or its application, feel free to call a factory Application Engineer at 716-684-0001 or your nearest PCB sales representative.

\subsection{ICP $^{\circledR}$ ACCELEROMETERS}

Powered by simple, inexpensive, constant-current signal conditioners, these sensors are easy to operate and interface with signal analysis, data acquisition and recording instruments. The following features further characterize ICP ${ }^{\circledR}$ sensors:

- Fixed voltage sensitivity, regardless of cable type or length.

- Low-impedance output signal, which can be transmitted over long cables in harsh environments with virtually no loss in signal quality.

- Two-wire operation with low cost coaxial cable, twoconductor ribbon wire or twisted-pair cabling.

- Low-noise, voltage-output signal compatible with standard readout, signal analysis, recording, and data acquisition equipment.

- Low cost per-channel - ICP ${ }^{\circledR}$ accelerometers require only an inexpensive, constant-current signal conditioner to operate.

\footnotetext{
${ }^{\mathbb{B}}$ ICP is a registered trademark of PCB Group, Inc., which uniquely identifies PCB sensors that incorporate built-in microelectronics.
}

- Intrinsic self-test feature - monitoring the sensor's output bias voltage provides an indication of proper operation, faulty condition, and bad cables.

In the rear of this manual you will find a Specification Sheet, which provides the complete performance characteristics of your particular sensor.

\subsection{OPTIONAL FEATURES}

Many sensors are supplied with standard, optional features. When listed before the model number, the following prefix letters indicate that the sensor is manufactured or supplied with a particular optional feature: "A" option: adhesive mount; "HT" option: extended high temperature range; "J" option: electrically ground isolated; "M" option: metric mounting thread; "Q" option: extended discharge time constant; "T" option: built-in transducer electronic data sheet (TEDS); and "W" option: attached, water-resistant cabling. Other prefix letters, such as "K", "KR", "GK", "GKR", "KL", and "GKL", indicate that the sensor is ordered in kit form, including interconnect cabling and signal conditioner. If you have any questions or concerns regarding optional features, consult the Vibration Division's product catalog or contact a PCB factory representative.

\subsection{INSTALLATION OVERVIEW}

When choosing a mounting method, consider closely both the advantages and disadvantages of each technique. Characteristics like location, ruggedness, amplitude range, accessibility, temperature, and portability are extremely critical. However, the most important and often overlooked consideration is the effect the mounting technique has on the high-frequency performance of the accelerometer. 
Shown in figure 1 are six possible mounting techniques and their effects on the performance of a typical piezoelectric accelerometer. (Note that not all of the mounting methods may apply to your particular sensor). The mounting configurations and corresponding graph demonstrate how the high-frequency response of the accelerometer may be compromised as mass is added to the system and/or the mounting stiffness is reduced.

NOTE: The low-frequency response is unaffected by the mounting technique. This roll-off behavior is typically fixed by the sensor's built-in electronics. However, when operating AC-coupled signal conditioners with readout devices having an input impedance of less than one megohm, the low frequency range may be affected. If necessary, contact a factory representative for further assistance.

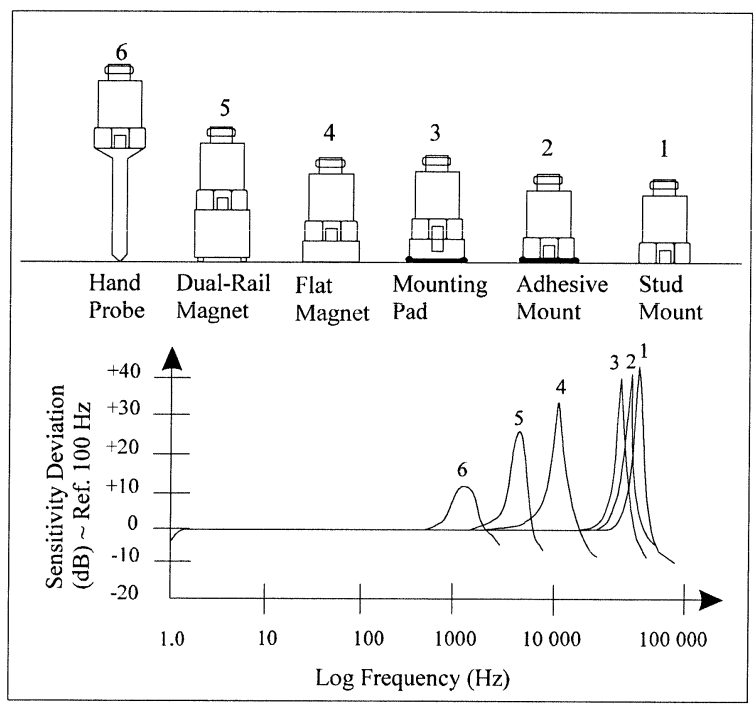

Figure 1. Assorted Mounting Configurations and Their Effects on High Frequency

\subsection{STUD MOUNT}

This mounting technique requires smooth, flat contact surfaces for proper operation and is recommended for permanent and/or secure installations. Stud mounting is also recommended when testing at high frequencies.

NOTE: Do NOT attempt mounting on curved, rough, or uneven surfaces, as the potential for misalignment and limited contact surface may significantly reduce the sensor's upper operating frequency range.

STEP 1: First, prepare a smooth, flat mounting surface, then drill and tap a mounting hole in the center of this area as shown in Figure 2 and in accordance with the enclosed Installation Drawing.

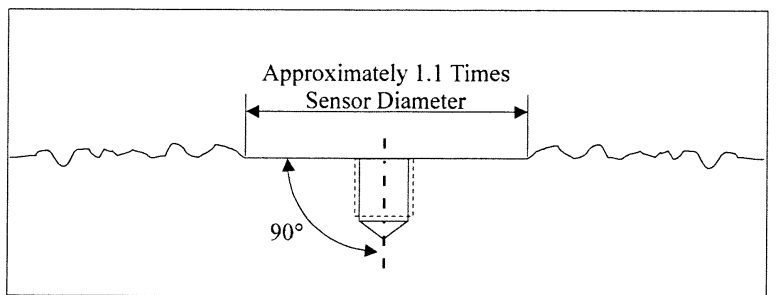

Figure 2. Mounting Surface Preparation

A precision-machined mounting surface with a minimum finish of $63 \mu \mathrm{in}(0.00016 \mathrm{~mm})$ is recommended. (If it is not possible to properly prepare the test structure mounting surface, consider adhesive mounting as a possible alternative). Inspect the area, checking that there are no burrs or other foreign particles interfering with the contact surface.

STEP 2: Wipe clean the mounting surface and spread on a light film of grease, oil, or similar coupling fluid prior to installation.

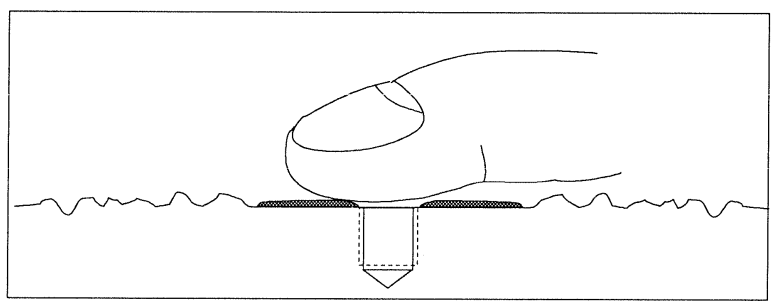

Figure 3. Mounting Surface Lubrication

Adding a coupling fluid improves vibration transmissibility by filling small voids in the mounting surface and increasing the mounting stiffness. For semipermanent mounting, substitute epoxy or another type of adhesive.

STEP 3: Screw the mounting stud into the base of accelerometer and hand-tighten (this step is unnecessary for units having an integral mounting stud). Then, screw the sensor into the tapped hole that was prepared in the test object. Tighten the unit in place by applying, with a torque wrench, the recommended mounting torque, as listed on the enclosed Installation Drawing.

NOTE: It is important to use a torque wrench during this step. Under-torquing the sensor may not adequately couple the device; over-torquing may result in stud failure.

\subsection{ADHESIVE MOUNT}

Adhesive mounting is often used for temporary installation or when the test object surface cannot be adequately prepared for stud mounting. Adhesives like hot glue and wax perform well for temporary installations whereas two-part epoxies and quick-bonding gels (super glue) provide a more permanent installation. Two 
techniques are used for adhesive mounting; they are via an adhesive mounting base (method 1 below) or direct adhesive mounting (method 2 below).

NOTE: Adhesively mounted sensors often exhibit a reduction in high-frequency range. Generally, smooth surfaces and stiff adhesives provide the best high frequency response.

\section{METHOD 1 - Adhesive Mounting Base}

This method involves attaching a base to the test structure, then securing the sensor to the base. This allows for easy removal of the accelerometer. Also, since many bases are manufactured of "hard-coated" aluminum, they provide electrical isolation to eliminate ground loops and reduce electrical interference that may propagate from the surface of the test object.

STEP 1: Prepare a smooth, flat mounting surface. A minimum surface finish of $63 \mu$ in $(0.00016 \mathrm{~mm})$ generally works best.

STEP 2: Stud-mount the sensor to the flat side of the appropriate adhesive mounting base according to the guidelines set forth in STEPS $\mathbf{2}$ and $\mathbf{3}$ of the Stud Mount Procedure presented above.

STEP 3: Place a small portion of adhesive on the underside of the mounting base (the underside is discernable by the concentric grooves which are designed to accept the adhesive). Firmly press down on the assembly to displace any extra adhesive remaining under the base.

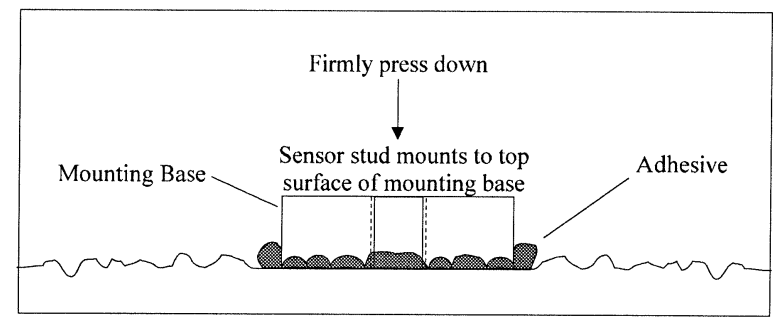

Figure 4. Mounting Base: Adhesive Installation

\section{METHOD 2 - Direct Adhesive Mount}

For restrictions of space or for convenience, most sensors can be adhesive-mounted directly to the test structure (an exception being units having integral mounting studs).

STEP 1: Prepare a smooth, flat mounting surface. A minimum surface finish of $63 \mu$ in $(0.00016 \mathrm{~mm})$ generally works best.

STEP 2: Place a small portion of adhesive on the underside of the sensor. Firmly press down on the top of the assembly to displace any adhesive. Be aware that excessive amounts of adhesive can make sensor removal difficult. Also, adhesive that may invade the tapped mounting hole in the base of the sensor will compromise future ability to stud mount the unit.

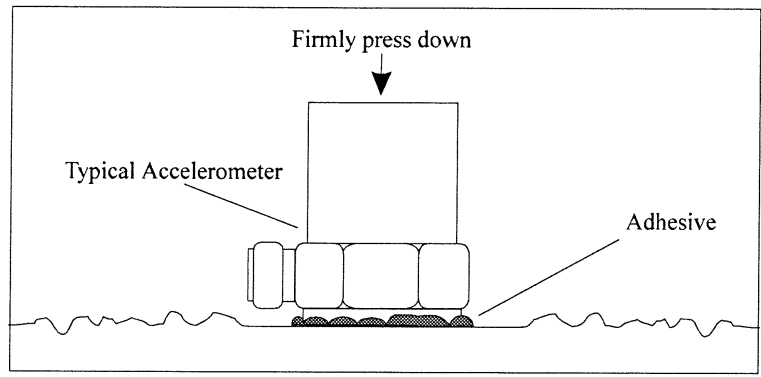

Figure 5. Direct Adhesive Mounting

\section{2-1 ADHESIVE MOUNT REMOVAL (other than wax)}

NOTE: A debonder should always be used to avoid sensor damage.

To avoid damaging the accelerometer, a debonding agent must be applied to the adhesive prior to sensor removal. With so many adhesives in use (everything from super glues, dental cement, epoxies, etc), there is no universal debonding agent available. The debonder for the Loctite 454 adhesive that PCB offers is Acetone. If you are using anything other than Loctite 454 , you will have to check with the individual manufacturers for their debonding recommendations. The debonding agent must be allowed to penetrate the surface in order to properly react with the adhesive, so it is advisable to wait a few minutes before removing the sensor.

After the debonding agent has set, you can use an ordinary open-end wrench if the accelerometer has a hex base or square base, or the supplied removal tool for teardrop accelerometers. After attaching either, use a gentle shearing (or twisting) motion (by hand only) to remove the sensor from the test structure.

\subsection{MAGNETIC MOUNT}

Magnetic mounting provides a convenient means for making quick, portable measurements and is commonly used for machinery condition monitoring, predictive maintenance, spot checks, and vibration trending applications.

NOTE: The correct magnet choice and an adequately prepared mounting surface are critical for obtaining reliable measurements, especially at high frequencies. Poor installations can cause as much as a 50\% drop in the sensor frequency range.

Not every magnet is suitable for all applications. For example, rare earth magnets are commonly used because 
of their high strength. Flat magnets work well on smooth, flat surfaces, while dual-rail magnets are required for curved surfaces such as motor housings and pipes. In the case of non-magnetic or rough surfaces, it is recommended that the user first weld, epoxy, or otherwise adhere a steel mounting pad to the test surface. This provides a smooth location for mounting and a target to insure that subsequent measurements for trending purposes are taken at the same location.

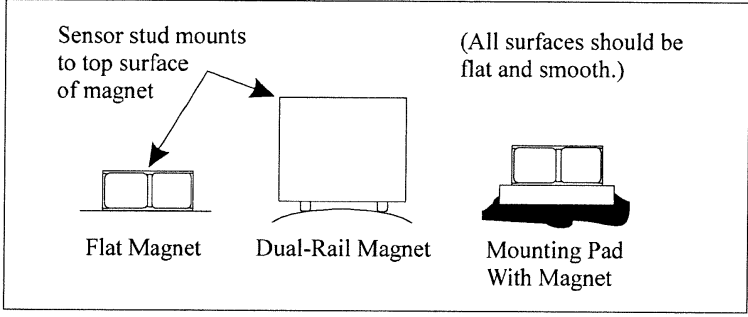

Figure 6. Magnet Types

STEP 1: Prepare a smooth, flat mounting surface. A minimum surface finish of $63 \mu$ in $(0.00016 \mathrm{~mm})$ generally works best. After cleaning the surface and checking for burrs, apply a light film of silicone grease, machine oil, or similar-type coupling fluid.

STEP 2: After choosing the correct magnet type, inspect the magnet, verifying that its mounting surfaces are flat and smooth.

STEP 3: Stud-mount the accelerometer to the appropriate magnet according to the guidelines set forth in STEP 3 of the above Stud Mount Procedure.

STEP 4: To avoid damage to the sensor, install the magnet/sensor assembly to the prepared test surface by gently "rocking" or "sliding" it into place.

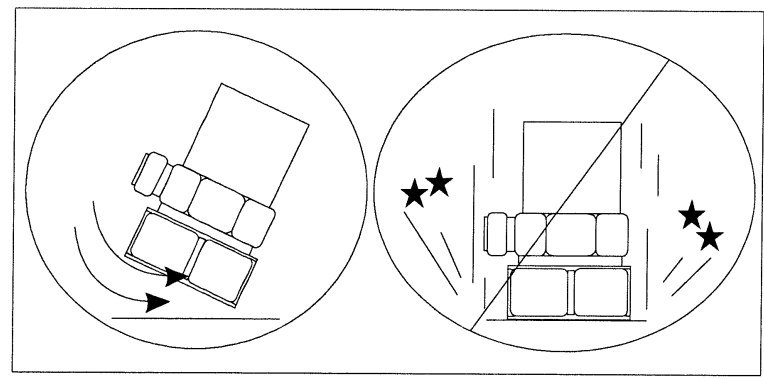

Figure 7. Magnet Mounting

CAUTION: Magnetically mounting of an accelerometer has the potential to generate very high (and very damaging) acceleration (g) levels. To prevent such damage, exercise caution and install the assembly gently by rocking it into place. If shock is expected to be a particular concern, use a sensor with built-in shock protection. For further assistance, contact a factory representative.

\subsection{HANDHELD OR PROBE TIP MOUNT}

This method is NOT recommended for most applications. Both the accuracy and repeatability at low $(<5 \mathrm{~Hz})$ and high frequency $(>1 \mathrm{kHz})$ ranges are questionable. It is generally used only for machinery condition monitoring, when installation space is restricted, or other portable trending applications. The technique, however, can be useful for initially determining locations of greatest vibration to establish a permanent sensor installation point.

\subsection{CABLING}

Care and attention to cable installation and cable condition is essential as the reliability and accuracy of any measurement system is no better than that of its weakest link. Do to the nature of vibration measurements, all sensor cables will ultimately fatigue and fail. Good installation practice will extend the life of a cable, however, it is highly recommended to keep spare cables on hand to enable continuation of the test in the event of a cable failure.

STEP 1: Ascertain that you have the correct cable type.

One cable type cannot satisfy all applications. ICP $^{\circledR}$ sensors can be operated with any ordinary two-wire or coaxial cable. Special, low-noise cables that are typically recommended for use with high-impedance, charge-output sensors can also be used. For applications requiring conformity to $C \epsilon$, low noise cables are essential. Industrial applications often require shielded, twisted-pair cables to reduce the effects of EMI and RFI that is present near electrical motors and machinery. Teflon-jacketed cabling may be necessary to withstand corrosive environments and higher temperatures. Consult the Vibration Division's product catalog for more information about cables or feel free to contact a factory representative for a specific recommendation on cables that are best suited for your application.

STEP 2: Connect the cable to the accelerometer.

A small amount of thread-locking compound placed on the connector threads prior to attachment helps secure the cable during testing. In wet, oily, or dirty environments, the connection can be sealed with silicone rubber sealant, O-rings, and flexible, heat-shrink tubing.

Coaxial Cables: Make connection by inserting the cable's connector pin into the sensor's mating socket. Then thread the connector into place by turning the cable connector's outer shell onto the accelerometer's electrical connector.

NOTE: Do not spin the accelerometer while holding the cable connector stationary, as this will cause undue 
friction on the center pin of the cable connector and lead to premature fatigue.

Multi-pin connectors: Make connection by inserting the sensor's mating pins onto the cable connector's mating sockets. Then thread the connector into place by turning the cable connector's outer shell onto the accelerometer's electrical connector.

Pigtail Connections: Certain miniature accelerometers and shock sensors are provided with lightweight cables attached to "Pigtail" connections. This type of connection reduces overall weight and incidence of connection intermittency under shock conditions. In the event of a cable or connection failure, the cables may be repaired in the field simply by re-soldering the stripped leads to the exposed pins on the sensor. (Check the Installation Drawing to determine signal and ground pins). In many cases, it is also helpful to protect the solder joint with heat-shrink tubing or epoxy.

NOTE: If you do not have the experience or resources to attach pigtail leads, consult PCB to discuss factory attachment. Damage to internal electronics may be caused by excessive heat during soldering and such failure is not covered by warranty.

STEP 3: Route the cable to the signal conditioner, making certain to relieve stress on the sensor/cable connection. Also, minimize cable motion by securing it with tape, clamps or ties at regular intervals.

Common sense should be used to avoid physical damage and minimize electrical noise. For instance, avoid routing cables near high-voltage wires. Do not route cables along floors or walkways where they may be stepped on or become contaminated. To avoid ground loops, shielded cables should have the shield grounded at one end only, typically at the signal conditioner.

STEP 4: Finally, connect the remaining cable end to the signal conditioner. It is good practice to dissipate any electrical charge that may have accumulated in the cable by shorting the signal pin to the ground pin or shell prior to attachment.

\subsection{POWERING}

All ICP ${ }^{\circledR}$ sensors require constant current excitation for proper operation. For this reason, use only PCB constantcurrent signal conditioners or other approved constantcurrent sources. A typical system schematic is shown in Figure 8.

NOTE: Damage to the built-in electronics resulting from the application of incorrect power, or the use of an unapproved power source, is NOT covered by warranty.

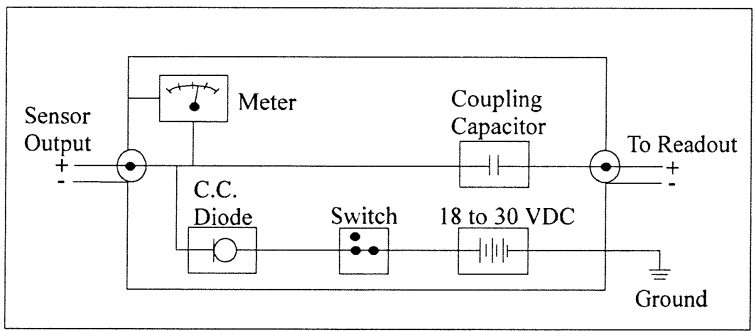

Figure 8. Typical System Schematic

The power supply consists of a current-regulated, 18 to 30 VDC source. This power is regulated by a currentlimiting circuit, which provides the constant-current excitation required for proper operation of $\mathrm{ICP}^{\circledR}$ sensors. In general, battery-powered devices offer versatility for portable, low-noise measurements, whereas line-powered units provide the capability for continuous monitoring. Consult the Vibration Division's product catalog for more information about signal conditioners.

NOTE: Under no circumstances should a voltage be supplied to an ICP ${ }^{\circledR}$ accelerometer without a currentregulating diode or equivalent electrical circuit. This may include ohmmeters, multi-meters and continuity testers.

Meters or LEDs are used on PCB signal conditioners to monitor the bias voltage on the sensor output signal, to check sensor operation, and detect cable faults. Normally, a "yellow" reading indicates an open circuit; "green" indicates normal operation; and "red" indicates either a short or overload condition. Finally, a capacitor at the output stage of the device removes the sensor output bias voltage from the measurement signal. This provides a zero-based, AC-coupled output signal that is compatible with most standard readout devices.

NOTE: Units having a low bias voltage may be in the "red," when actually they are working properly. If suspect, the bias voltage can be checked with a voltmeter attached to a " $T$ " connector installed on the input connector to the signal conditioner.

Note: For readout devices having an input impedance near one gigohm (as encountered with some $A$ to $D$ converters), it may be necessary to place a one megohm resistor in parallel to the readout input to eliminate slow turn-on and signal drift.

Today, many FFT analyzers, data acquisition modules, and data collectors have the proper constant-current excitation built-in for direct use with $\mathrm{ICP}^{\circledR}$ sensors. Before using this feature, however, check that the supply voltage and constant current are within acceptable limits for use with your particular sensor. (Check enclosed Specification Sheet). Please contact the respective signal 
conditioner manufacturer or check the product manual for more information.

\subsection{OPERATING}

After completing the system setup, switch on the signal conditioner and allow 1 to 2 minutes for the system to stabilize. The meter (or LED) on the signal conditioner should be reading "green." This indicates proper operation and you may begin taking measurements. If a faulty condition is indicated (red or yellow reading), first check all system connections, then check the functionality of the cable and signal conditioner. If the system still does not operate properly, consult a PCB factory representative.

NOTE: Always operate the accelerometer within the limitations listed on the enclosed Specification Sheet. Operating the device outside these parameters can cause temporary or permanent damage to the sensor.

\subsection{ACCELEROMETER CALIBRATION}

Accelerometer calibration provides, with a definable degree of accuracy, the necessary link between the physical quantity being measured and the electrical signal generated by the sensor. In addition, other useful information concerning operational limits, physical parameters, electrical characteristics, or environmental influences may also be determined. Without this link, analyzing data becomes a nearly impossible task. Fortunately, most sensor manufacturers provide a calibration record that documents the exact characteristics of each sensor. (The type and amount of data varies depending on the manufacturer, sensor type, contractual regulations, and other special requirements).

Under normal conditions, piezoelectric sensors are extremely stable, and their calibrated performance characteristics do not change over time. However, the sensor may be temporarily or permanently affected by harsh environments influences or other unusual conditions that may cause the sensor to experience dynamic phenomena outside of its specified operating range. This change manifests itself in a variety of ways, including: a shift of the sensor resonance due to a cracked crystal; a temporary loss of low-frequency measuring capability due to a drop in insulation resistance; or total failure of the built-in microelectronic circuit due to a high mechanical shock.

For these reasons, it is recommended that a recalibration cycle be established for each accelerometer. This schedule is unique and is based on a variety of factors, such as: extent of use, environmental conditions, accuracy requirements, trend information obtained from previous calibration records, contractual regulations, frequency of "crosschecking" against other equipment, manufacturer recommendation, and any risk associated with incorrect readings. International standards, such as ISO 10012-1, provide insight and suggest methods for determining recalibration intervals for most measuring equipment. With the above information in mind and under "normal" circumstances, PCB conservatively suggests a 12 - to 24month recalibration cycle for most piezoelectric accelerometers.

NOTE: It is good measurement practice to verify the performance of each accelerometer with a Handheld Shaker or other calibration device before and after each measurement. The PCB Handheld Shaker operates at a fixed frequency and known amplitude $(1.0 \mathrm{~g})$ to provide a quick check of sensor sensitivity.

\subsection{RECALIBRATION SERVICE}

PCB offers recalibration services for our piezoelectric accelerometers, as well as units produced by other manufacturers. Our internal metrology laboratory is certified to ISO 9001, accredited by A2LA to ANSI/IEC 17025 and ANSI/NCSL Z540-1, complies with ISO 10012-1 (and former MIL-STD-45662A), and uses equipment directly traceable to NIST. Our investment in equipment, traceability and conformance to industry standards ensures accurate calibration against relevant specifications, in a timely fashion.

\subsection{BACK-TO-BACK CALIBRATION THEORY}

Many companies choose to purchase the equipment necessary to perform the recalibration procedure in house. While this may result in both a savings of time and money, it has also been attributed to incorrect readings and costly errors. Therefore, in an effort to prevent the common mistakes associated with customer-performed calibration, this document includes a broad overview of the Back-to-Back Calibration technique. This technique provides a quick and easy method for determining the sensitivity of a test accelerometer over a wide frequency range.

Back-to-Back Calibration is perhaps the most common method for determining the sensitivity of piezoelectric accelerometers. This method relies on a simple comparison to a previously calibrated accelerometer, typically referred to as a reference standard.

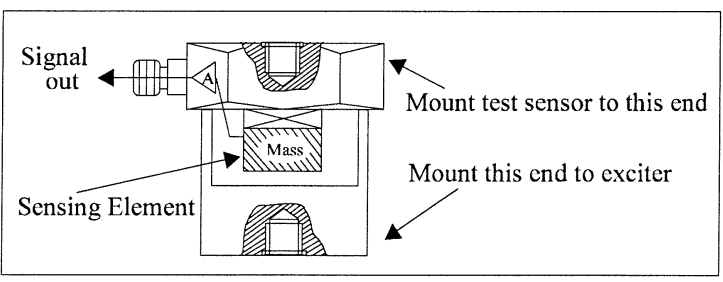

Figure 9. Reference Standard Accelerometer 
These high-accuracy devices, which are directly traceable to a recognized standards laboratory, are designed for stability, as well as configured to accept a test accelerometer. By mounting a test accelerometer to the reference standard and then connecting this combination to a suitable vibration source, it is possible to vibrate both devices and compare the data as shown in Figure 10. (Test set-ups may be automated and vary, depending on the type and number of accelerometers being calibrated).

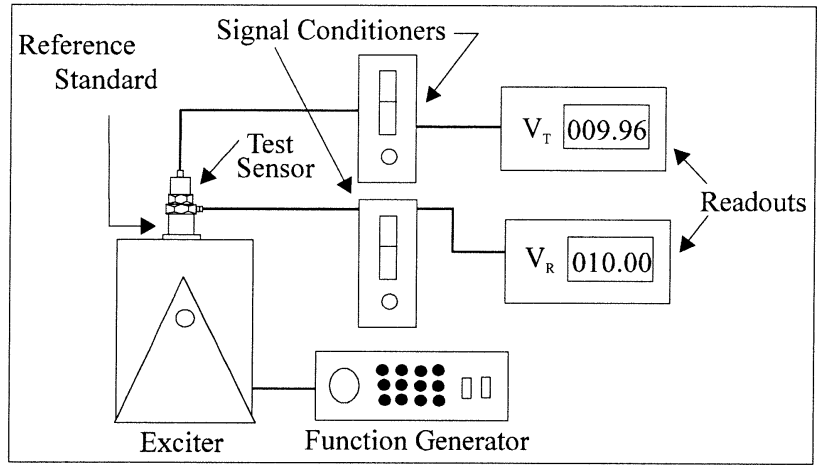

Figure 10. Typical Back-to-Back Calibration System

Because the acceleration is the same on both sensors, the ratio of their outputs $\left(\mathrm{V}_{\mathrm{T}} / \mathrm{V}_{\mathrm{R}}\right)$ must also be the ratio of their sensitivities. With the sensitivity of the reference standard $\left(S_{R}\right)$ known, the exact sensitivity of the test sensor $\left(\mathrm{S}_{\mathrm{T}}\right)$ is easily calculated by using the following equation:

$$
\mathrm{S}_{\mathrm{T}}=\mathrm{S}_{\mathrm{R}}\left(\mathrm{V}_{\mathrm{T}} / \mathrm{V}_{\mathrm{R}}\right)
$$

By varying the frequency of the vibration, the sensor may be calibrated over its entire operating frequency range. The typical response of an unfiltered accelerometer is shown in Figure 11.

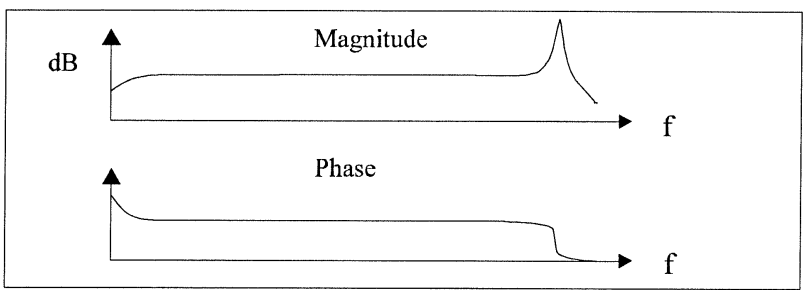

Figure 11. Typical Test Accelerometer Response

\subsection{PCB CALIBRATION PROCEDURE}

Numerous precautions are taken at PCB to insure accurate and repeatable results. This section provides a brief overview of the primary areas of concern.

Since the Back-to-Back Calibration technique relies on each sensor experiencing an identical acceleration level, proper mounting of the test sensor to the reference standard is imperative. Sensors with mounting holes are attached directly to the reference standard with a stud tightened to the recommended mounting torque. A shouldered mounting stud is typically used to prevent the stud from "bottoming out" in the hole. Both mounting surfaces are precision-machined and lapped to provide a smooth, flat interface according to the manufacturer's specification. A thin layer of silicone grease is placed between the mating surfaces to fill any imperfections and increase the mounting stiffness. The cables are stressrelieved by first routing them to the shaker head, then to a nearby stationary location. This reduces cable motion, which is especially important when testing charge output sensors, and helps to prevent extraneous motion or stresses from being imparted into the system. A typical set-up is shown in Figure 12.

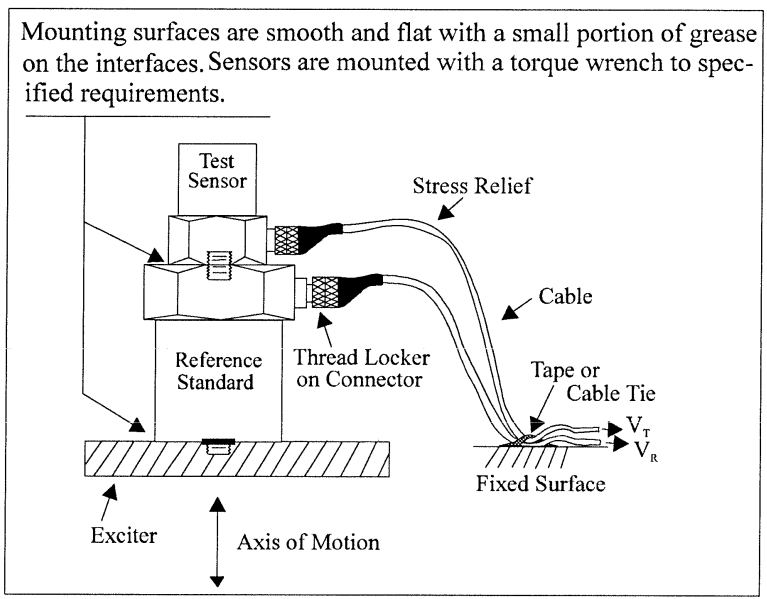

Figure 12. Typical Calibration Set-Up

Adhesively mounted sensors use similar practices. However, in this case, a small portion of quick-bonding gel, or similar temporary adhesive, is used to attach the test sensor to a reference standard designed with a smooth, flat mounting surface.

In addition to mounting, the selection of the proper equipment is critical. Some of the more important considerations include: 1) the reference standard must be specified and previously calibrated over the frequency and/or amplitude range of interest; 2) the shaker should be selected to provide minimal transverse (lateral) motion and minimal distortion; and 3) the quality of the meters, signal generator, and other devices should be selected so as to operate within the limits of permissible error.

\subsection{COMMON MISTAKES}

Most calibration errors are caused by simply overlooking some of the fundamental principals of dynamics. This section attempts to address some of the more common concerns.

For stud-mount sensors, always mount the accelerometer directly to the reference standard. Ensure that the mounting surfaces are smooth, flat, and free of any burrs. Always use a 
coupling fluid, such as silicone grease, in the mounting interface to maintain a high mounting stiffness. Mount the sensor according to the manufacturer's recommended mounting torque. DO NOT use any intermediate mounting adaptors, as the mounted resonant frequency may be reduced, and thereby compromise the high-frequency performance. If necessary, use adaptor studs.

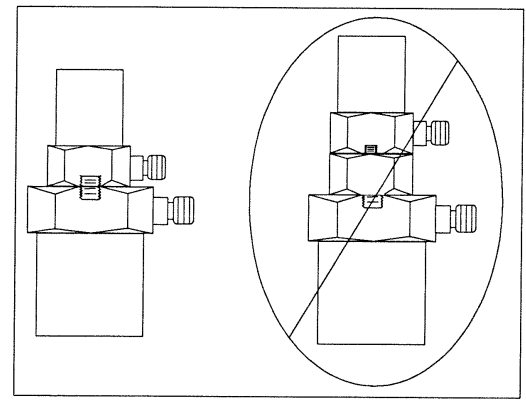

Figure 13. Stud Mounting

For adhesive mount sensors, use a thin, stiff layer of temporary adhesive such as quick-bonding gel or superglue. DO NOT use excessive amounts of glue or epoxy, as the mounting stiffness may be reduced and compromise highfrequency performance. It may also damage the sensor during removal.

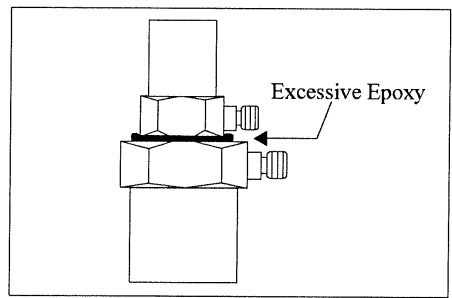

Figure 14. Incorrect Adhesive Mounting

Triaxial accelerometers should always be mounted directly to the reference standard. Unless absolutely required, DO NOT use adaptors to re-orient the sensor along the axis of motion, as the mounting stiffness may be altered. The vibration at the test sensor's sensing element may differ from the vibration at the reference standard due to a "cantilever" effect, seen in Figure 15.

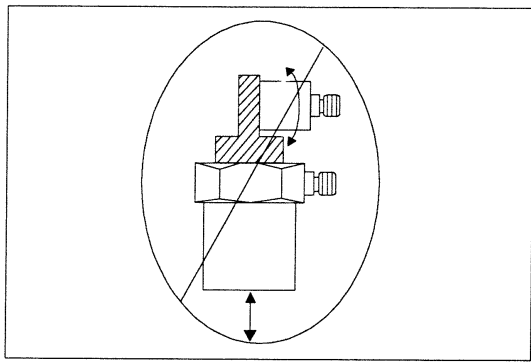

Figure 15. Mounting Triaxial Sensors (Incorrect)

Understand Back-to-Back Calibration limitations. Do not expect the uncertainty of calibration to be any better than $\pm 2 \%$. (In fact, the uncertainty may be as high as $\pm 3 \%$ or $\pm 4 \%$ for frequencies $<10 \mathrm{~Hz}$ or $>2 \mathrm{kHz}$.) Since large sensors may affect high-frequency accuracy, verify that the test sensor does not mass load the reference standard. Validate your calibration system with another accelerometer prior to each calibration session. Check with the manufacturer for exact system specifications.

\subsection{CONCLUSIONS}

Without an adequate understanding of dynamics, determining what, when, and how to test a sensor is a difficult task. Therefore, each user must weigh the cost, time, and risk associated with self-calibration versus utilizing the services of an accredited laboratory.

\subsection{SERVICE}

See the supplement sheet, contained in this manual, for information on our warranty, service, repair, and return policies and instructions.

When unexpected measurement problems arise, call our 24-hour SensorLine ${ }^{\mathrm{SM}}$ to discuss your immediate dynamic instrumentation needs with a factory representative.

Dial 716-684-0001. 


\section{TPCB PIEZOTRONISS vesarano omsion}

3425 Walden Avenue, Depew, NY 14043-2495 USA Vibration Division toll-free 888-684-0013

24-hour SensorLine ${ }^{\text {SM }}$ 716-684-0001 FAX 716-685-3886 E-mail vibration@pcb.com Website www.pcb.com

\section{A PCB GROUP COMPANY ISO 9001 CERTIFIED A2LA ACCREDITED to ISO 17025}

(C) 2002 PCB Group, Inc. In the interest of constant product improvement, specifications are subject to change without notice. PCB and ICP are registered trademarks of PCB Group, Inc. SensorLine is a service mark of PCB Group, Inc. All other trademarks are properties of their respective owners. 


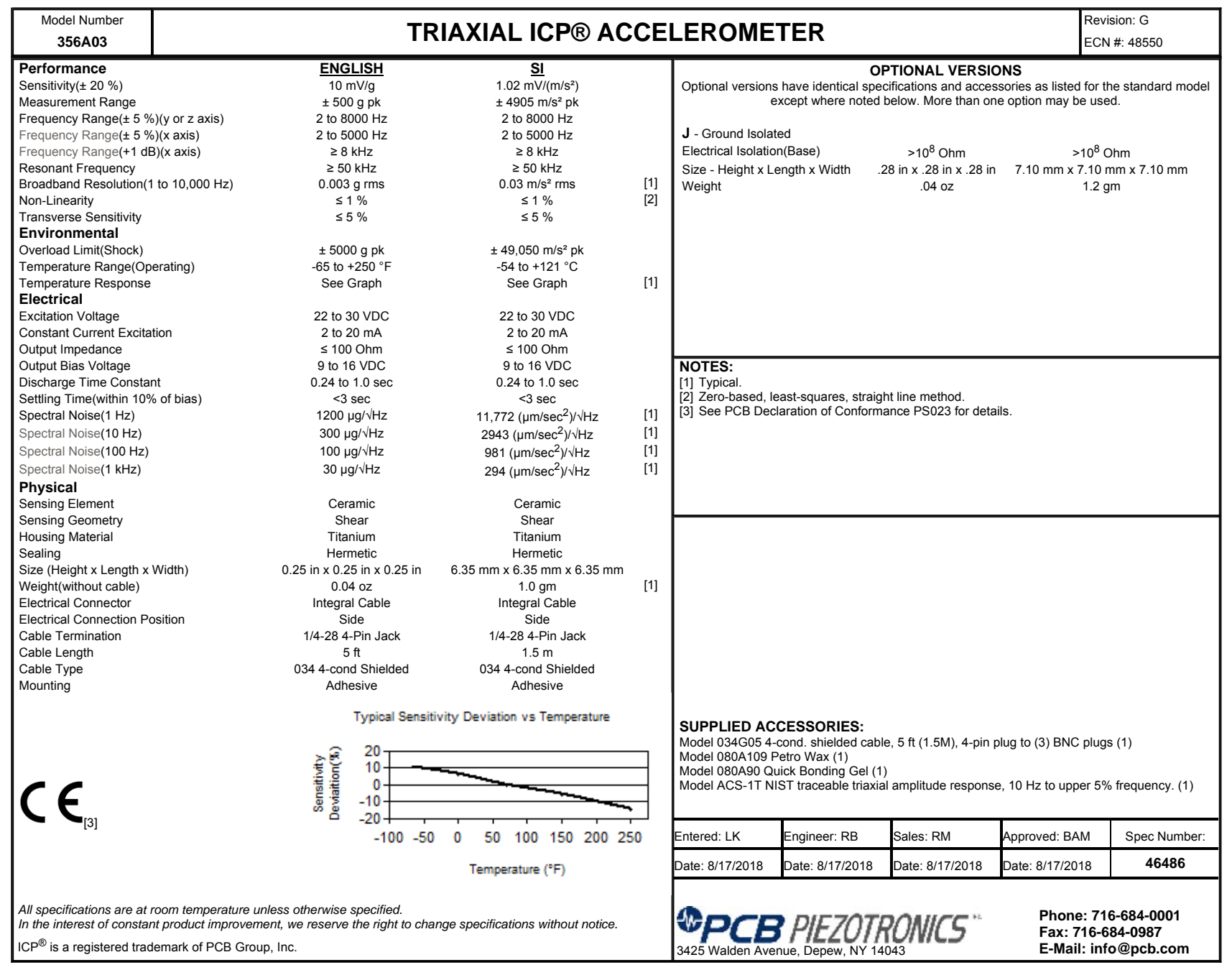




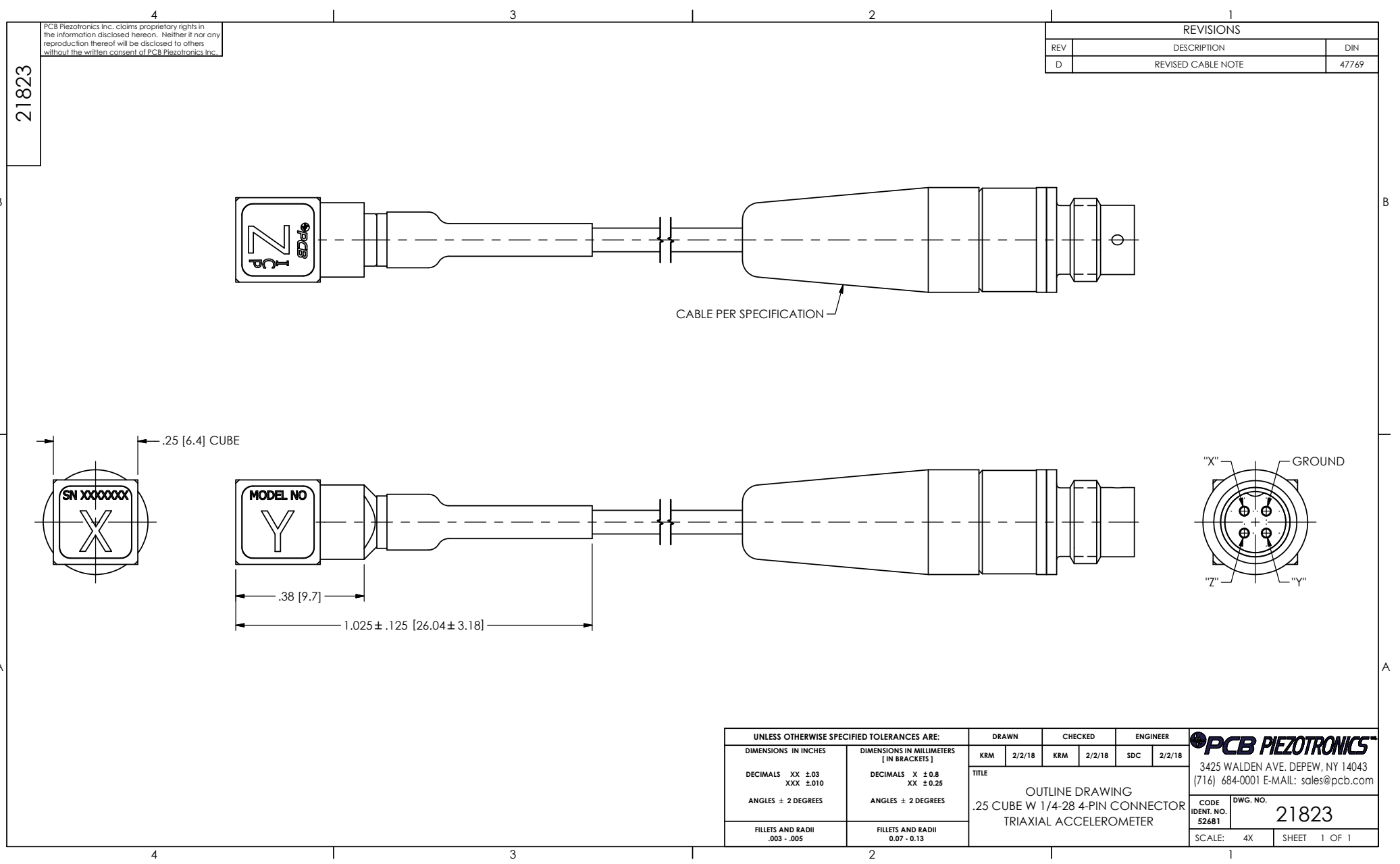


Appendix D

MESH CONVERGENCE STUDIES

Table D.1: Mesh convergence study of the test pod with a fixed base. A seed size of $0.004 \mathrm{~m}$ was selected for the FEA model.

\begin{tabular}{|c|c|c|c|c|c|c|c|}
\hline \multirow{2}{*}{ Mode } & \multicolumn{70}{|c|}{ Natural Frequency (Hz) } \\
\cline { 2 - 8 } & $\begin{array}{c}\mathbf{0 . 0 2} \mathbf{~ m} \\
\text { seed }\end{array}$ & $\begin{array}{c}\mathbf{0 . 0 1} \mathbf{~ m} \\
\text { seed }\end{array}$ & $\begin{array}{c}\mathbf{0 . 0 0 7} \mathbf{~ m} \\
\text { seed }\end{array}$ & $\begin{array}{c}\mathbf{0 . 0 0 5} \mathbf{~ m} \\
\text { seed }\end{array}$ & $\begin{array}{c}\mathbf{0 . 0 0 4} \mathbf{~ m} \\
\text { seed }\end{array}$ & $\begin{array}{c}\mathbf{0 . 0 0 3} \mathbf{~ m} \\
\text { seed }\end{array}$ & $\begin{array}{c}\mathbf{0 . 0 0 2} \mathbf{~ m} \\
\text { seed }\end{array}$ \\
\hline 1 & 1127.5 & 1056.5 & 1041.7 & 1029.5 & 1025.0 & 1015.8 & 936.11 \\
\hline 2 & 1440.9 & 1215.2 & 1194.4 & 1200.4 & 1174.9 & 1172.4 & 1028.6 \\
\hline 3 & 1632.1 & 1417.2 & 1423.3 & 1423.1 & 1420.3 & 1416.4 & 1160.9 \\
\hline 4 & 2151.8 & 1722.5 & 1678 & 1677 & 1628.9 & 1625.5 & 1391.2 \\
\hline 5 & - & 2401.2 & 2352.8 & 2355.1 & 2296.3 & 2293.3 & 1941.5 \\
\hline 6 & - & - & - & - & - & - & 2071.2 \\
\hline
\end{tabular}

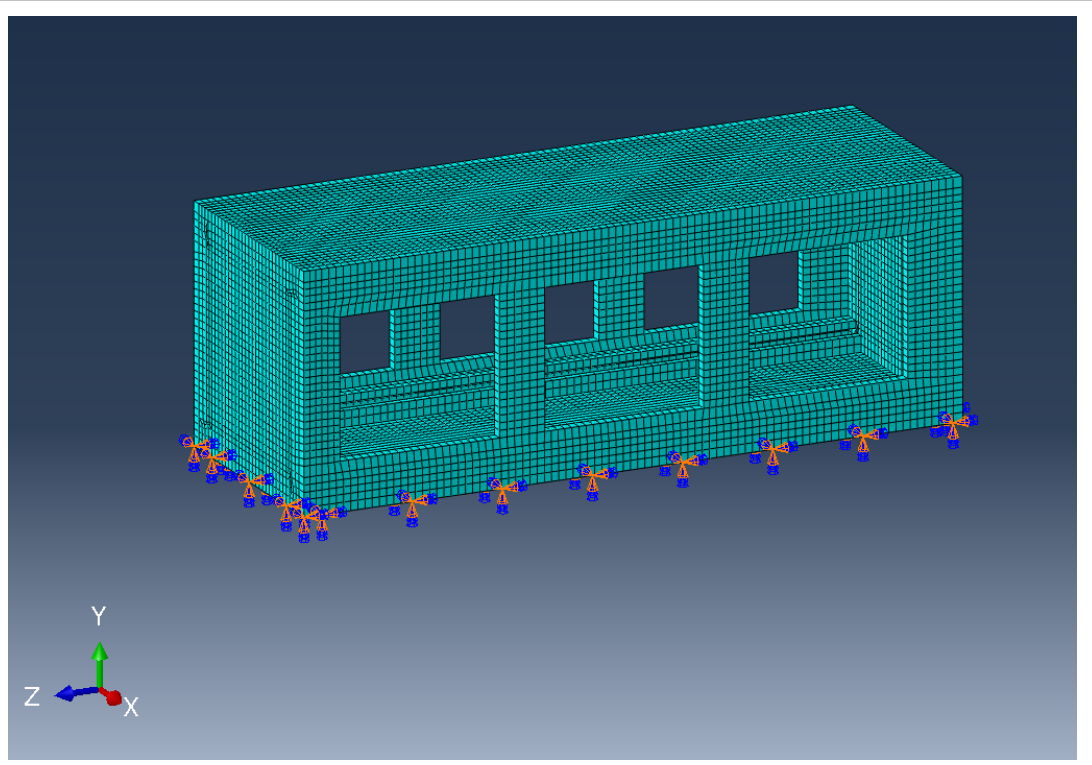


Table D.2: Mesh convergence study of the mass model with a fixed base. A seed size of $0.005 \mathrm{~m}$ was selected for the FEA model.

\begin{tabular}{|c|c|c|c|c|c|}
\hline \multirow{2}{*}{ Mode } & \multicolumn{5}{|c|}{ Natural Frequency (Hz) } \\
\cline { 2 - 6 } & $\begin{array}{c}\mathbf{0 . 0 2} \mathbf{~ m} \\
\text { seed }\end{array}$ & $\begin{array}{c}\mathbf{0 . 0 1} \mathbf{~ m} \\
\text { seed }\end{array}$ & $\begin{array}{c}\mathbf{0 . 0 0 7} \mathbf{~ m} \\
\text { seed }\end{array}$ & $\begin{array}{c}\mathbf{0 . 0 0 5} \mathbf{~ m} \\
\text { seed }\end{array}$ & $\begin{array}{c}\mathbf{0 . 0 0 4} \\
\text { seed }\end{array}$ \\
\hline 1 & 1369.8 & 1475.9 & 1482.5 & 1480.8 & 1479.3 \\
\hline 2 & 1372.5 & 1607.6 & 1614.7 & 1614.4 & 1613.7 \\
\hline 3 & 1374.2 & 1772.2 & 1776.9 & 1777.9 & 1777.5 \\
\hline 4 & 1375.5 & 2013.1 & 2024.3 & 2027.1 & 2027.7 \\
\hline 5 & 1375.5 & - & - & - & - \\
\hline 6 & 1376.7 & - & - & - & - \\
\hline 7 & $\ldots$ & - & - & - & - \\
\hline
\end{tabular}

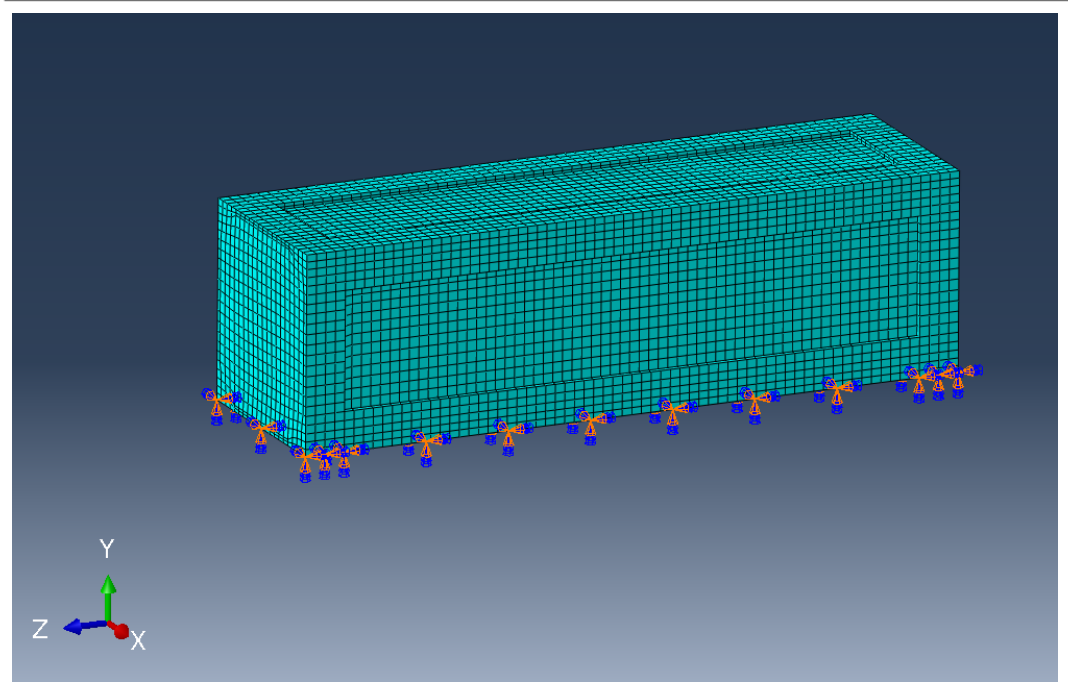


Table D.3: Mesh convergence study of the fixture plate with a fixed base. A seed size of $0.007 \mathrm{~m}$ was selected for the FEA model.

\begin{tabular}{|c|c|c|c|c|}
\hline \multirow{2}{*}{ Mode } & \multicolumn{4}{|c|}{ Natural Frequency (Hz) } \\
\cline { 2 - 5 } & $\begin{array}{c}\mathbf{0 . 0 2} \mathbf{~ m} \\
\text { seed }\end{array}$ & $\begin{array}{c}\mathbf{0 . 0 1} \mathbf{~ m} \\
\text { seed }\end{array}$ & $\begin{array}{c}\mathbf{0 . 0 0 7} \mathbf{~ m} \\
\text { seed }\end{array}$ & $\begin{array}{c}\mathbf{0 . 0 0 5} \mathbf{~ m} \\
\text { seed }\end{array}$ \\
\hline 1 & 11322 & 11419 & 11434 & 11434 \\
\hline 2 & 11390 & 11490 & 11503 & 11503 \\
\hline 3 & 11714 & 11847 & 11870 & 11876 \\
\hline 4 & 13010 & 13213 & 13246 & 13254 \\
\hline 5 & 13121 & 13354 & 13391 & 13402 \\
\hline
\end{tabular}

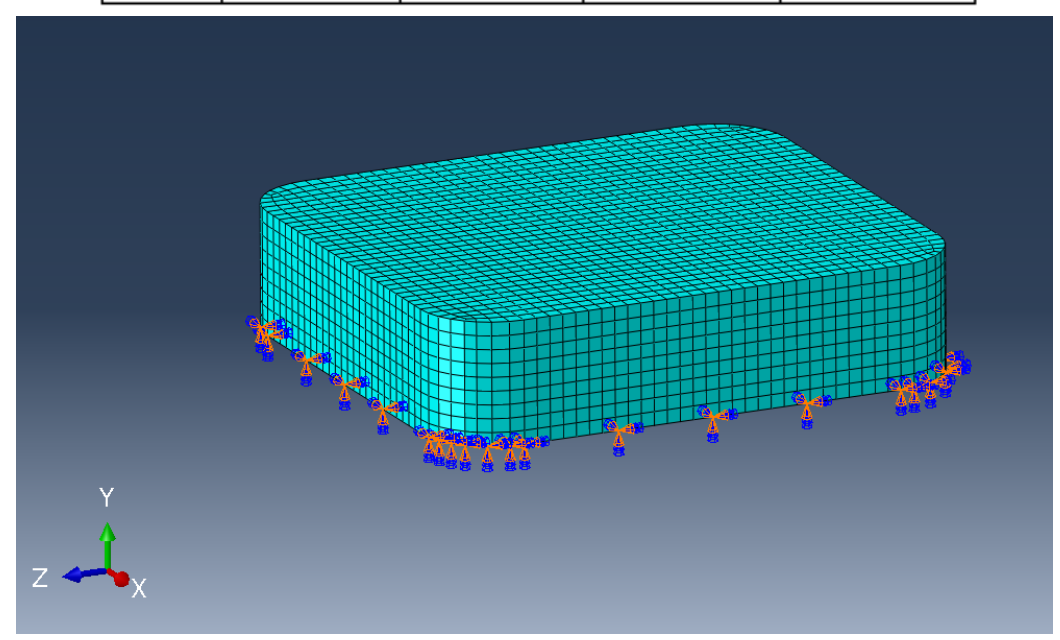

